Article

\title{
Thermodynamics of High Temperature Plasmas
}

\section{Ettore Minardi}

Istituto di Fisica del Plasma, Via R. Cozzi 53 I-20125 Milano Italy; E-mail: minardi@ifp.cnr.it

Received: 19 December 2008 / Accepted: 4 March 2009 / Published: 13 March 2009

\begin{abstract}
In this work we discuss how and to what extent the thermodynamic concepts and the thermodynamic formalism can be extended to the description of high temperature states of the plasma not necessarily associated with a Boltzmann distribution and with thermal equilibrium.The discussion is based on the "magnetic or electrostatic entropy concept", an interpretative and predictive tool based on probability and information, defined in a suitably coarse-grained possibility space of all current density or of all electric charge density distributions under testable constraints, and whose variation properties are proven to be related under certain conditions to the equilibrium and the stability of the system. In the case of magnetic equilibrium the potentiality of the magnetic entropy concept is illustrated by comparing the predictions of the current density and pressure profiles with the observations in different tokamak machines and different tokamak regimes, as well as by showing how the equilibrium and the stability in devices as different as the reversed field pinch or the magnetic well are described by the variation properties of the same entropy functional applied to the different situations. In fact it emerges that the maximum of the entropy can be seen in these different cases as an optimization constraint for the minimum of the magnetic energy. The application of the entropy concept to the electrostatic processes shows in particular that the so-called reactive instabilities (nondissipative, non-resonant instabilities with a marginal point) admit a neighboring state with higher entropy and are therefore of special relevance from the point of view of the physical evolution of the system. In this case the thermodynamic formalism allows the introduction of the concept of "thermodynamic fluctuations" of the macroscopic charge density and provides a method for the calculation of the "thermodynamic" fluctuation levels both on the stable as well as on the linearly unstable side of the marginal point. The paper discusses the relation between the variations of the entropy functional defined on statistical grounds and the motion of the underlying system of particles. It is found that the vanishing of the first variation of the entropy is connected, under certain assumptions, with the Hamilton's principle, while the second variation is not directly related to the dynamics but is an
\end{abstract}


expression of the fact that the entropy is a predictive tool based on probability and information.

Keywords: Thermodynamics of Plasmas; General Theory and Basic Studies; Tokamaks; Reversed Field Pinch; Magnetic Well; MHD and Electrostatic Instabilities.

PACS Codes: 52.55.Fa; 52.55.Dy; 52.25.Kn

\section{INDEX}

1. Introduction

CHAPTER 1: The statistical model of magnetic collisionless equilibria

2. The separation between collective and individual effects and the statistics of the plasma volume

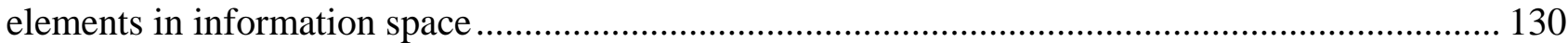

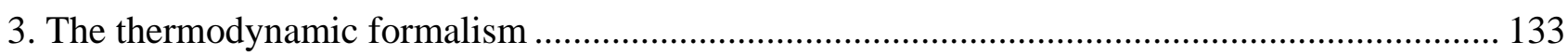

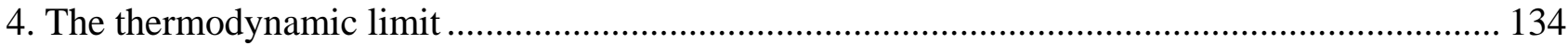

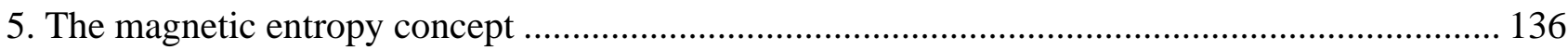

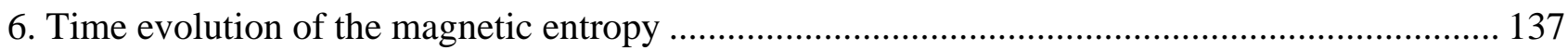

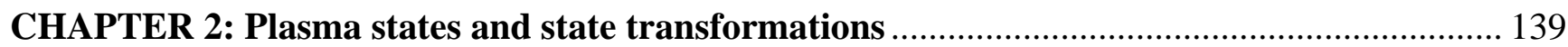

7. Stationary magnetic entropy (SME) and power balance of the tokamak................................. 139

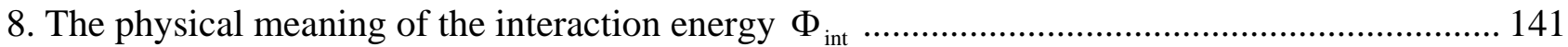

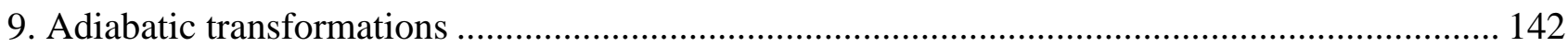

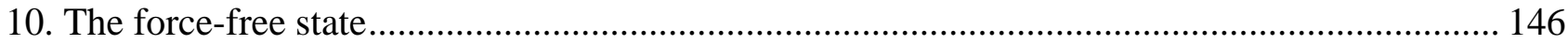

CHAPTER 3: Local entropy and local plasma properties ................................................. 148

11. Local entropy variations and local structure of the magnetic equilibrium.............................. 149

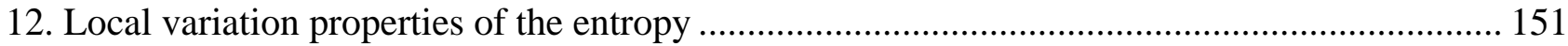

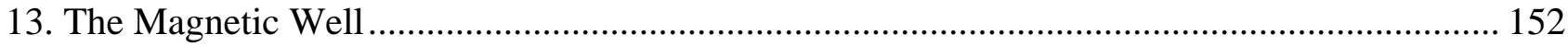

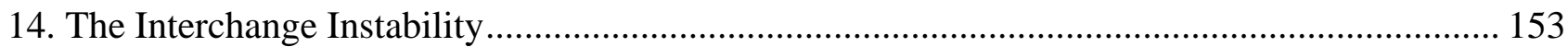

15. Thermodynamic interpretation of the tearing modes ................................................................ 155

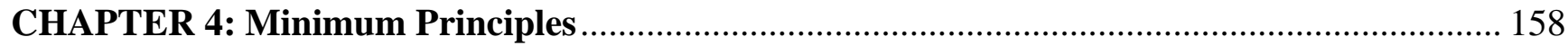

16. Minimum plasma thermal energy and pressure profile in tokamaks ..................................... 158

17. Minimum poloidal magnetic energy and profile consistency of the SME states ....................... 161

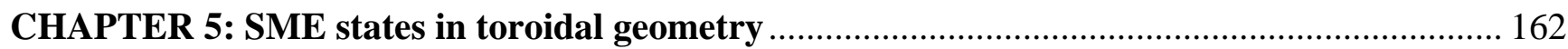

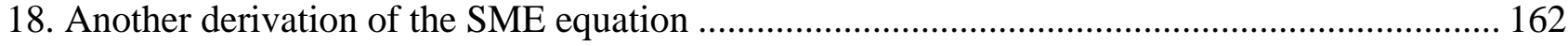

19. Conditions on the toroidal equilibrium of the tokamak …...................................................... 165 
CHAPTER 6: Comparisons between theory and experiment.................................................. 167

20. Comparisons with the experiment; case of ohmically relaxed currents ................................. 168

20.1-Current density, temperature and pressure profiles ..................................................... 168

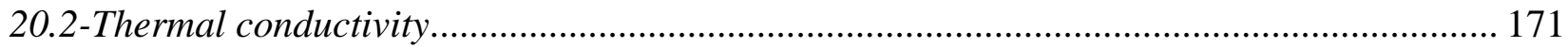

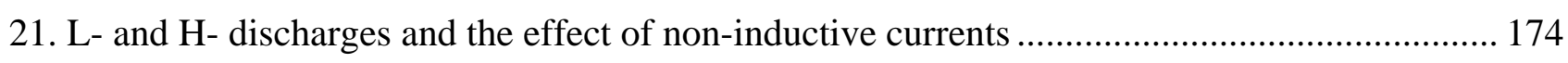

CHAPTER 7: Connexion between magnetic entropy and Lagrangian description of the particle motion 180

22. Lagrangian motion of the particles with macroscopic constraints .......................................... 181

23. The relation between the magnetic entropy and the Maxwellian average of the Lagrangian.... 184

24. The relation of the first variation of the magnetic entropy to the first variation of the canonically averaged Lagrangian and to the action principle...................................................................... 188

CHAPTER 8: Statistical model of electrostatic collisionless equilibria .................................... 191

25. Thermodynamic formalism for electrostatic collisionless equilibria ...................................... 192

26. The electrostatic entropy concept....................................................................................... 194

27. Physical meaning of the electrostatic interaction energy .................................................. 197

CHAPTER 9: Non-linear effects in reactive instabilities ...................................................... 199

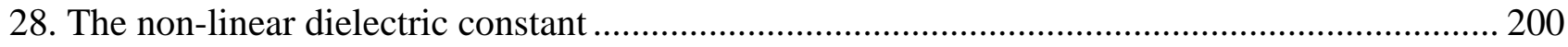

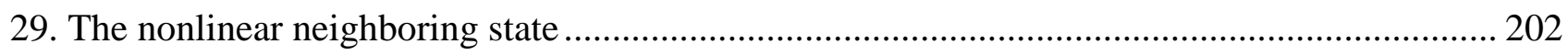

30. Probability distribution of charge fluctuations around the marginal equilibrium ..................... 204

CHAPTER 10: Thermodynamic fluctuation levels: examples.............................................. 206

31. Thermodynamic fluctuations in reactive purely electrostatic systems................................... 206

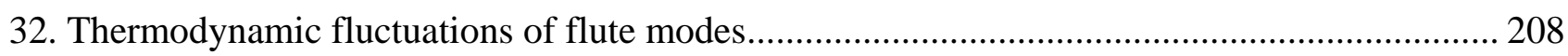

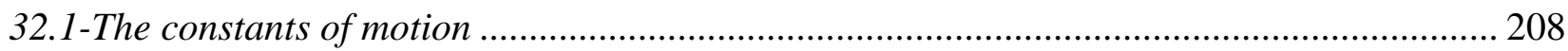

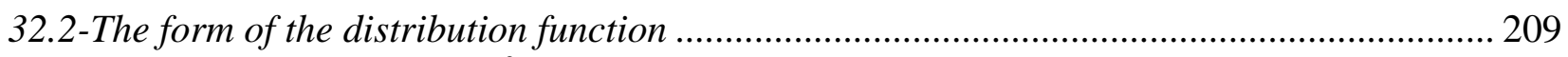

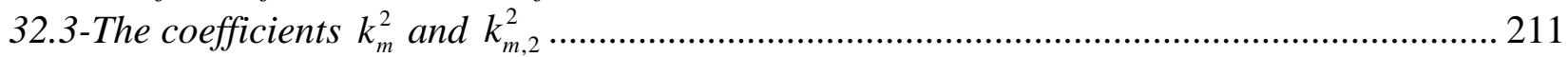

CHAPTER 11: Connexion between the electrostatic entropy and the Lagrangian description of

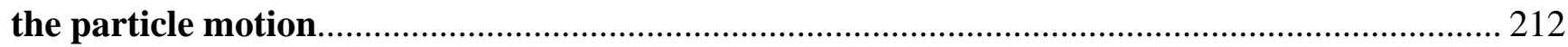

33. Coarse graining of the high temperature system of Coulomb interacting particles .................. 213

34. Equivalence of the first variations of the electrostatic entropy and of the canonically averaged

Lagrangian with respect to adiabatic variations ......................................................................... 215

35. Particle motion in the electrostatic system with the collective constraint and the relation of the

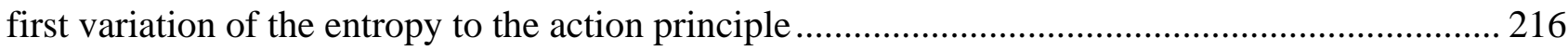




\section{Introduction}

We say that the plasma has a high temperature when the time scale of the processes taking place in it is much smaller than the collision time arising from the Coulomb interactions of its particle constituents.

In the high temperature plasma the term that takes into account the effects of the individual collisions in the time evolution of the plasma configuration in phase space described by the Boltzmann equation, is neglected. This term, in its various versions and implicit assumptions, determines the evolution of the plasma toward a uniquely defined stationary distribution in phase space, namely the Boltzmann distribution.

In the case of high temperature plasma one can set up a description based on a distribution function satisfying a conservation equation in a reduced 6-dimensional space, the so-called Vlasov equation. In the Vlasov picture all kinds of individual particle-particle correlations are neglected and only collective (macroscopic) effects involving organized cumulative motions of charged particles with the associated electric and magnetic fields are taken into account. A relevant consequence of this collective description is that the uniqueness of the stationary solution is lost because any arbitrary function of the constants of motion is a stationary solution of the Vlasov equation. This arbitrariness is not reduced when one restricts himself to the consideration of particular symmetric geometries, as the axissymmetric geometry of the tokamak, and of particular closure approximations as that of the Magnetohydrodynamics. In this case the equilibrium is described by the Grad-Shafranov equation, which depends on two arbitrary functions.

In contrast with this theoretical situation, it is an experimental fact that the magnetically confined plasma adopts well-defined equilibria, which, in some cases, exhibit a remarkable stiffness, particularly in the current density and in the pressure profiles. The question arises whether some general criterion can be found that selects a very limited set of high temperature states among the infinite possibilities allowed by the theory in its present form.

A possibility that occurs naturally to the mind is the existence of a functional of the equilibrium quantities, which select the collision-less states of the plasma according to their variation properties and which play the same formal role of the thermodynamic potentials of classical thermodynamics. In other words the question is whether a thermodynamic theory can be formulated of the static or quasistatic (i.e. very near to the marginal point of stability) collision-less states also in situations very far from the Boltzmann equilibrium.

The possibility of such a theory is related to the existence of a functional whose variation properties could characterize the collision-less states according to a hierarchy of probabilities and whose formal properties could be the same as that of the entropy of classical thermodynamics. Once this functional is constructed, the thermodynamic properties will eventually emerge after comparison of the formal properties of the functional with the concrete physical features of the collision-less states of the plasma. According to this point of view one defines the probability of a collective macroscopic state by operating in the possibility space of all current density distributions or electric charge density distributions (conveniently coarse-grained) and assigns the probability $P_{i}$ of each distribution (labeled 
by $i$ ) of a canonical ensemble by requiring that the entropy $S=-\sum_{i} P_{i} \ln \left(P_{i}\right)$ be stationary with respect to the variation of the $P_{i}$ under given constraints.

We shall consider a scheme where the collective system is so constrained that its collective energy (magnetic or electrostatic) is fixed, as well as its interaction energy with external sources of energy and electric charges. The latter is simulated by the interaction with an infinite background of independent particles (electrically neutral in the average) that acts as an infinite reservoir of energy and electric charges. The collective distributions of current density or electric charge density of the equilibrium states are introduced through the constraints as given but not "a priori" specified functions of space and the privileged collective states are determined by the variation properties of the entropy pertaining to the collective equilibrium integrated over the fluctuations of the background.

Subsequently, when the collision-less states of maximum entropy are determined, one can apply the classical relation between the variations of the entropy and the fluctuations for calculating the "thermodynamic" (in the sense that will be specified in the text) fluctuation levels of the collective system around the collective equilibrium state.

The development of a thermodynamic theory along the lines outlined above is the purpose of the present work. The result is the emergence of a set of conditions that strongly reduce the arbitrariness of the magnetic and electrostatic collision-less states of equilibrium, consistently with the experimental observations.

The thermodynamic point of view reveals unexpected relations concerning the stability of a dynamical system and the stability defined thermodynamically in accordance with the variation properties of the collective entropy in regimes as different as those of the plasma in the tokamak, in the reversed field-pinch or in a magnetic well.

In the case of electrostatic processes, the thermodynamic treatment points to the so called "reactive" instabilities (characterized by absence of dissipation and existence of bifurcation points) as the most relevant from the physical point of view, in view of the fact that they involve neighboring states of maximum entropy for critical values of the parameters.

Even though the entropy is not a dynamical quantity, but a predictive tool based on probability and information, a connexion can be found between the equilibrium described by the vanishing of the first variation of the entropy and the motion of the underlying system of particles described by the Hamilton action principle. Through the comparison with the Lagrangian description of motion at the single particle level, as well as at the macroscopic level, the quantities involved in the construction of the entropy find a precise physical interpretation and the determination of the scale length characterizing the domain of validity based on the entropy functional emerges clearly. However a direct connexion with the dynamics does not hold for the second variation of the entropy, which fact reflects the peculiar non-dynamical content of the entropy concept. Nevertheless, the structure of the entropy functional is such that the maximum of the entropy can be seen, in many significant cases, as an optimization constraint for the minimum of the energy (magnetic or electrostatic). In other words, the energy is minimized under the condition that the probability of the magnetic or electrostatic configuration be at a maximum.

Though independent of the detailed dynamics, the global point of view offered by the thermodynamics of high temperature plasmas can be hopefully useful, just as in the classical case, for 
unraveling those phenomenological parameters which are at the heart of the physical process and which are worthy of a more specific dynamical analysis.

\section{CHAPTER 1: The statistical model of magnetic collisionless equilibria}

Summary: We start with the definition of the information space, that is, of the possibility space of all current density distributions compatible with the information expressed by given constraints. The volume $V$ in ordinary space is partitioned into $N$ volume elements $\Delta V$ in which the macroscopic current density can be considered as uniform, so that $\Delta V$ measures the coarse-graining. The $N$ volume elements are the basic objects of the statistics. Each volume element $\Delta V_{j}$ is susceptible of occupying at random a position in the $6 N$-dimensional possibility space of all positions $\vec{x}_{i}$ in $V$ and of all current density values $\vec{J}_{i}$ at $\vec{x}_{i}$. The current density $\vec{J}_{i}$ is divided in two parts $\vec{J}_{j}=\vec{j}\left(\vec{x}_{j}\right)+\overrightarrow{\tilde{j}}_{j}$, where $\vec{j}(\vec{x})$ is a macroscopic current density due to collective motions of the electrons involving a scale length larger than $(\Delta V)^{1 / 3}$ and defined in a volume $\Omega$ that satisfies the inequalities $\Delta V<<\Omega<<$.

The electrons carry the current and the ions form a uniform neutralizing background. The part $\vec{j}_{j}$ is due to the velocity fluctuations around the average value of individual electrons that can be considered as effectively free inside each $\Delta V_{j}$ provided $\Delta V_{j}$ is so small that one can ignore the collective interactions between many particles inside it. The concept of separation between electromagnetic or electrostatic collective and individual effects described by characteristic lengths, goes back to old papers by Bohm and Pines [1-2] and will play a natural central role in our formulation.

The partition of the current density is introduced through the following two independent constraints in information space:

1) The sum of the collective magnetic energy $(1 / 2 c) \int \vec{j} \cdot \vec{A} d \Omega$, and of the interaction energy of the collective vector potential $\vec{A}$ with the background of fluctuating currents $(1 / 2 c) \int \vec{j} \cdot \vec{A} d \Omega$, is prescribed.

2) The dispersion of the current density $\vec{j}(r)+\overrightarrow{\widetilde{j}}$ arising from the fluctuating part $\overrightarrow{\tilde{j}}$ is fixed. This is the same as to fixing the temperature of a "heat bath" modeled by the background of electrons effectively free in each $\Delta V_{j}(1 \leq j \leq N)$, covering the whole volume $\left.V>>\Omega\right)$. In this scheme the individual particle-particle correlation is neglected. Indeed, this approximation is the basis of the derivation of the Vlasov equation from Liouville equation and of the description of collective collision-less processes. However, while the particle-particle interaction is neglected, the interaction of the current fluctuations due to the individual particles with the "smeared out" collective field is taken into account and will be seen to vanish only for the isolated system at thermodynamic equilibrium. In fact, the interaction energy with the background of independent particles will prove to be a useful device for simulating the reversible interaction of the collective system with external sources of energy.

The entropy $S=-\sum_{i} P_{i} \ln \left(P_{i}\right)$ follows from the requirement to be stationary with respect to variations of the $P_{i}$ under the constraints above. 
In the thermodynamic limit, $N / \Omega \rightarrow \infty$ with $\Delta V$ fixed the entropy $S$ turns out to be divided in a part $S_{p}$ called "magnetic entropy" that depends only on the collective quantities of the plasma equilibrium and in a part $S_{b}$ related to the infinite background of individually uncorrelated particles in interaction with the collective field.

When the plasma is enclosed in a perfectly conductive shell and the quantities of the collective equilibrium vary slowly in time, the time derivative of $S_{p}$, calculated with the help of the Maxwell equations and with a suitable modeling of the image currents in the shell, is expressed in terms of the inductive electric field generated internally to the plasma. When an external electric field has penetrated into the plasma through narrow cuts in the shell and the global plasma configuration is stationary, the entropy $S_{p}$ is also stationary. The condition that the plasma is stationary locally leads to an equation for the current density profile $\vec{j}(\vec{x})[3]$.

\section{The separation between collective and individual effects and the statistics of the plasma volume elements in information space}

For tackling the question outlined in the introduction one must define, as a first step, a suitable space $\Gamma$ where all possible current density distributions can be represented and where the information on the system can be introduced in the form of constraints on these distributions. We adopt the Bayesian point of view of information theory [4] where the constraints have a hypothetical meaning. That is to say, one advances testable constraints that imply a determination (an assignment) of a probability distribution $P$ by maximizing the entropy $S=-\int_{\Gamma} P \ln P d \Gamma$ with respect to variations of $P$ under the constraints to be tested. If the probability $P$ and the entropy, calculated with these constraints, prove to be consistent with the observations, then one infers the physical consistency of the constraints and, at the same time, the internal consistency of the theory. In our specific case the existence of a given macroscopic current density distribution will be introduced ' $a$ priori' through 'testable' constraints whose probability assignment in the space of all possible current density distributions is determined by the entropy principle and is to be confirmed by the experiment.

We then proceed to define the space $\Gamma$ where all our information on the system will be expressed. The volume $V$ of the plasma is partitioned into a large number $N$ of volume elements in which the macroscopic current density can be considered as uniform, so that $\Delta V=V / N$ measures the coarsegraining through which the plasma will be seen. The size of $\Delta V$ is subject to lower and upper limits. The lower limit arises from the requirement that the number of particles contained in $\Delta V$ be very large. At the same time $(\Delta V)^{1 / 3}$ should not be larger than the screening length below which the particles can be regarded as effectively free and can be treated individually while the collective effects dominate at scale length larger than $(\Delta V)^{1 / 3}$. We shall meet the precise value of the screening length, according to the calculations of Bohm and Pines [1-2] in the course of development of the theory (chapter 7, section 23, chapter 11 section 33). At present we assume that, at scale lengths larger then the coarse-graining described by $\Delta V$, only collective effects be visible and that, compatibly with the conditions above, $\Delta V$ can be chosen so small that the collective quantities can be taken as uniform in it. 
The current density $\vec{J}_{i}$ can be divided in two parts $\left.\vec{J}_{j}=\vec{j}_{(} \vec{x}_{j}\right)+\vec{j}_{j}$, where $\vec{j}(\vec{x})$ is a macroscopic current density due to collective motions of the electrons involving a scale length larger than $(\Delta V)^{1 / 3}$ and is defined in a volume $\Omega$ that satisfies the inequalities $\Delta V<<\Omega<<V$ and is vanishing outside $\Omega$.

The electrons carry the current and the ions form a uniform neutralizing background. The part $\overrightarrow{\widetilde{j}}_{j}$ is due to the velocity fluctuations around the average value of the effectively free electrons inside each $\Delta V_{j}$ where $\Delta V_{j}$ satisfies the limits given above. In view of the fluctuating part $\vec{j}, \vec{J}_{j}$ is a random variable.

We consider an assembly formed by the large number $N$ of volume elements $\Delta V$; the elements $\Delta V$ are the basic objects of our statistics. Let us introduce a six-dimensional space for the values of the current density $\vec{J}$ and for the position $\vec{x}$ in the volume $V$. We now take one volume element from the assembly, call it $\Delta V_{j}$ and place it at random in a copy of the above six-dimensional space denoted by $S_{j}$. The volume element will be at a certain position, denoted by $\left(\vec{x}_{j}, \vec{J}_{j}\right)$ (where $\vec{x}_{j}$ is for instance the center of $\left.\Delta V_{j}\right)$. We do the same for another volume element $\Delta V_{i}$, in another space $S_{i}$, taking care that $\Delta V_{i}$ can only occupy in $S_{i}$ a position $\vec{x}_{i} \neq \vec{x}_{j}$ because the volume elements cannot overlap in $V$. After repeating this procedure for all $N$ volume elements, one obtains a single point in a $6 N$-dimensional space $\Gamma$ which is the Cartesian product of all the $S_{j}$. This point represents a particular current density distribution of the plasma, reconstructed in the volume $V$ with a coarse-graining $\Delta V$.

The space $\Gamma$ is called 'information space' and the current density $\vec{J}_{j}$ is the 'information variable' (other examples of information variables are the charge density. in the case of the electrostatic plasma equilibrium and the mass density in the case of the gravitational equilibrium). Our purpose is to calculate the probability $P\left(\vec{J}_{1} \ldots \vec{J}_{N} ; \vec{x}_{1} \ldots \vec{x}_{N}\right)$ for the assembly of $N$ volume elements to occupy at random any given volume element $d \Gamma$ in information space. To see the form of $d \Gamma$, we note that the volume accessible in 3-dimensional space to the volume element $j=1$, is $V=N \Delta V$. The volumes accessible to the volume elements $j=2$ and $j=3$ are $V-\Delta V=(N-1) \Delta V$, and $V-2 \Delta V=(N-2) \Delta V$, and so on. Thus the volume accessible to the assembly of $N$ volume elements is

$(N(N-1)(N-2) \ldots 1)(\Delta V)^{N}=N ! \Delta V^{N}$.

This must be divided by $N$ ! because a permutation among the $N$ volume elements has no physical effect and must not contribute to the total volume element in information space. Hence $d \Gamma$ is given by $d \Gamma=\Delta V^{N} d \vec{J}_{1} \ldots d \vec{J}_{N}$.

Further constraints are now imposed on the part of information space that concerns the current density distributions.

As we said, the total current density in each $\Delta V_{j}$ is formed by the coarse-grained contribution (uniform in $\Delta V_{j}$ ) of a macroscopic (or collective) current density $\vec{j}\left(\vec{x}_{j}\right)$ and by a fluctuating part $\overrightarrow{\widetilde{j}}_{j}$ arising from the global effect of the particle discreteness in $\Delta V_{j}$. The part $\vec{j}\left(\vec{x}_{j}\right)$, related to the collective equilibrium, is not specified 'a priori”, as it is an unknown to be determined later from the variation properties of the entropy. The total current density $\vec{J}_{j}=\vec{j}\left(\vec{x}_{j}\right)+\overrightarrow{\tilde{j}}_{j}$, where $-\infty \leq \overrightarrow{\tilde{j}}_{j} \leq+\infty$, is a random variable in view of its fluctuating part. The $\overrightarrow{\tilde{j}}_{j}$ are the integration variables, while $\vec{j}\left(x_{j}\right)$ can be treated as a constant. Additionally, $\vec{j}\left(x_{j}\right)$ is taken as vanishing outside a volume $\Omega<<$ while 
$\vec{j} \neq 0$ everywhere in $V$. The quantities $V$ and $N$ will that tend eventually to infinity with fixed $\Delta V$ and $\Omega$ (thermodynamic limit: see section 3 ).

We adopt a scheme in which the plasma configuration is characterized by the magnetic energy interaction of the current density $\vec{J}$ with the part $\vec{A}(\vec{x})$ of the vector potential created only by the collective part $\vec{j}(\vec{x})$ of the total current density $\vec{J}$. That is to say we neglect the interaction of the fluctuating part $\overrightarrow{\tilde{j}}$ with itself. Then we put

$\frac{1}{2 c} \int_{\Gamma} P\left(\vec{J}_{1} \ldots \vec{J}_{N} ; \vec{x}_{1} \ldots \vec{x}_{N}\right) \sum_{j=1}^{N}\left(\overrightarrow{\tilde{j}}_{j}+\vec{j}\left(\vec{x}_{j}\right)\right) \cdot \vec{A}\left(\vec{x}_{j}\right) \Delta V_{j} d \Gamma=\Phi_{\text {int }}+\Phi$.

In view of the normalization condition

$\int_{\Gamma} P\left(\vec{J}_{1} \ldots \vec{J}_{N} ; \vec{x}_{1} \ldots \vec{x}_{N}\right) d \Gamma=1$,

the equation (1.3) can be separated in the parts

$\Phi_{\text {int }}=\frac{1}{2 c} \int_{\Gamma} P\left(\vec{J}_{1} \ldots \vec{J}_{N} ; \vec{x}_{1} \ldots \vec{x}_{N}\right) \sum_{j=1}^{N} \overrightarrow{\widetilde{j}}_{j} \cdot \vec{A}\left(\vec{x}_{j}\right) \Delta V_{j} d \Gamma$

$\Phi=\frac{1}{2 c} \sum_{j=1}^{N} \vec{j}\left(\vec{x}_{j}\right) \cdot \vec{A}\left(\vec{x}_{j}\right) \Delta V_{j}$.

Here $\Phi_{\text {int }}$ is the energy of the interaction between the current fluctuations of the background and the collective potential of the macroscopic configuration.

In the derivation of the Vlasov equation from the Liouville equation the particle-particle correlations are neglected and the Vlasov equilibrium is collisionless. Consistently with this picture the fluctuating part $\widetilde{\tilde{j}}_{j}$ arises from the velocity fluctuations of particles that can be treated as individually independent in each $\Delta V_{j}$, covering the whole volume $V$.

Pure Vlasov equilibria are uncorrelated to the fluctuations arising from particle discreteness. This fact should be expressed in our scheme by equating to zero the particle-collective field interaction energy $\Phi_{\text {int }}$. However the assumption of a non-vanishing energy interaction of the individual particle belonging to the fluctuating background in each $\Delta V_{j}$ with the "smeared out" collective field will prove to be a useful device for simulating the complicated external interactions that tend to drive the collective (macroscopic) equilibrium outside the pure Vlasov equilibrium. Indeed, $\Phi_{\text {int }}$, when calculated explicitly in the formalism that we are developing, will play a crucial role for the description of the energy emitted or absorbed by the collective system, through the Poynting flux of electromagnetic energy (see section 8). In other words the interaction of the collective system with the fluctuating background simulates the interaction of this system with the external world. We then consider the equation (2.3) as a constraint to the maximization procedure of the entropy, where $\Phi_{\text {int }}+\Phi$ is fixed and $\Phi$ and $\Phi_{\text {int }}$ are to be determined later according to the physical situation at hand.

A further constraint on $P$ arises from the fact that $\vec{J}$ is a random variable, whose values are then subject to dispersion. It is convenient to characterize the dispersion by fixing the value $\overline{\Delta J^{2}}$ of the following average quadratic form

$\overline{\Delta J^{2}}=\frac{1}{N} \int_{\Gamma} P\left(\vec{J}_{1} \ldots \vec{J}_{N} ; \vec{x}_{1} \ldots \vec{x}_{N}\right) \sum_{j=1}^{N} \vec{J}_{j}^{2} d \Gamma$. 
We shall see in section 4 that this form is consistent in the proper limit (the thermodynamic limit) with the conventional definition $\overline{J_{i}^{2}}-\bar{J}_{i}^{2}$ of the local variance in each $\Delta V_{i}$ (see eq. (4.9)). It is not necessary to specify now the value of $\overline{\Delta J^{2}}$. This value will be found in section 23 while discussing the relation of the present model to the dynamics of the underlying system of particles.

The probability $P$ can now be determined from the requirement that the entropy $S=-\int_{\Gamma} P \ln P d \Gamma$

be stationary with respect to arbitrary variations of $P$ under the three constraints (2.3), (2.4) and (2.7).

\section{The thermodynamic formalism}

Applying the technique of the Lagrange multipliers we are led to find the extreme of the functional $\mathcal{S}=-\int_{\Gamma} P \ln P d \Gamma-\alpha \int_{\Gamma} P \sum_{j=1}^{N} \vec{J}_{j}^{2}-\frac{1}{\tau C} \int_{\Gamma} P \sum_{j=1}^{N} \vec{J}_{j} \cdot \vec{A}\left(\vec{x}_{j}\right) \Delta V_{j} d \Gamma-\gamma \int_{\Gamma} P d \Gamma$,

where $\alpha, \gamma$ and $\tau$ are constants to be determined later in order to satisfy the constraints. The vanishing of the first variation of $S$ with respect to arbitrary variations of $P$ gives

$P=P_{0} \exp \left(-\alpha \sum_{j=1}^{N} \vec{J}_{j}^{2}-\frac{1}{\tau c} \sum_{j=1}^{N} \vec{J}_{j} \cdot \vec{A}\left(\vec{x}_{j}\right) \Delta V_{j}\right)$,

where $P_{0}=\exp (-\gamma-1)$. Let us put

$\alpha \vec{J}_{j}^{2}+\frac{1}{\tau} \vec{J}_{j} \bullet \vec{A}\left(\vec{x}_{j}\right) \Delta V_{j}=\alpha\left(\vec{J}_{j}-\overrightarrow{\vec{J}}_{j}\right)^{2}+C_{j}$,

where $\overrightarrow{\vec{J}}_{j}$ and $C_{j}$ are independent of $\vec{J}_{j}$ and are given by

$\left(\bar{J}_{k}\right)_{j}=-\frac{1}{2 \alpha \tau c} A_{k}\left(\vec{x}_{j}\right) \Delta V_{j}, \quad C_{j}=-\frac{1}{4 \alpha \tau^{2} c^{2}} \sum_{k=1}^{3} A_{k}^{2} \Delta V_{j}^{2}$,

where $k$ denotes the components of $\vec{J}_{j}$ and $\vec{A}$. The $\Gamma$-integration then reduces to integration over Gaussian distributions. Recalling the normalization condition (2.4), we obtain

$$
\begin{aligned}
& P=\frac{\exp \left(-\alpha\left(\sum_{j=1}^{N}\left(\vec{J}_{j}-\overrightarrow{\vec{J}}_{j}\right)^{2}\right)\right)}{\Delta V^{N}(\pi / \alpha)^{3 N / 2}}=\frac{\exp \left(-\alpha\left(\sum_{j=1}^{N}\left(\overrightarrow{\widetilde{j}}_{j}-\overline{\vec{j}}_{j}\right)^{2}\right)\right)}{\Delta V^{N}(\pi / \alpha)^{3 N / 2}}, \\
& P_{0}=\frac{\exp \left(\sum_{j=1}^{N} C_{j}\right)}{\Delta V^{N}(\alpha / \pi)^{3 N / 2}} .
\end{aligned}
$$

One can verify the relations

$$
\begin{aligned}
& \overline{\vec{J}}_{i}=\vec{j}\left(\vec{x}_{i}\right)+\overline{\vec{\jmath}}_{j}=\int_{\Gamma} P \vec{J}_{i} d \Gamma=-\frac{1}{2 \alpha \tau c} \vec{A}\left(\vec{x}_{i}\right) \Delta V_{i}, \\
& \Phi_{\text {int }}=\frac{1}{2 c} \sum_{j=1}^{N} \overline{\vec{j}}_{j} \cdot \vec{A}\left(\vec{x}_{j}\right) \Delta V_{j}=-\Phi-\frac{\Delta V^{2}}{4 \alpha \tau c^{2}} \sum_{j=1}^{N} \vec{A}^{2}\left(\vec{x}_{j}\right) \\
& \overline{\vec{J}}_{i}^{2}=\int_{\Gamma} P \vec{J}_{i}^{2} d \Gamma=\frac{3}{2 \alpha}+\overrightarrow{\vec{J}}_{i}^{2}=\frac{3}{2 \alpha}+\frac{\Delta V^{2}}{4 \alpha^{2} \tau^{2} c^{2}} \vec{A}^{2}\left(\vec{x}_{i}\right),
\end{aligned}
$$


$\overline{\Delta J^{2}}=\frac{1}{N} \sum_{j=1}^{N} \int_{\Gamma} P \vec{J}_{j}^{2} d \Gamma=\frac{3}{2 \alpha}+\frac{\Delta V^{2}}{4 \alpha^{2} \tau^{2} c^{2} N} \sum_{j=1}^{N} \vec{A}^{2}\left(\vec{x}_{j}\right)$

Combining (3.7d) with (3.7b), we obtain

$\alpha=\frac{1}{\overline{\Delta J^{2}}}\left(\frac{3}{2}-\frac{1}{N \tau}\left(\Phi+\Phi_{\text {int }}\right)\right)$,

$\tau=-\frac{\Delta V}{4 \alpha c^{2}} \frac{\sum_{j=1}^{N} \vec{A}^{2}\left(\vec{x}_{j}\right) \Delta V_{j}}{\Phi+\Phi_{\text {int }}}$.

We can now proceed to the calculation of the entropy:

$S=-\int_{\Gamma} P \ln P d \Gamma=N \ln \left(\Delta V\left(\frac{\pi}{\alpha}\right)^{3 / 2}\right)+\alpha \int_{\Gamma} P \sum_{j=1}^{N}\left(\vec{J}_{j}-\overline{\vec{J}}_{j}\right)^{2} d \Gamma=$

$=N \ln \left(\Delta V\left(\frac{\pi}{\alpha}\right)^{3 / 2}\right)+\alpha N \overline{\Delta J^{2}}-\frac{\Delta V^{2}}{4 \alpha \tau^{2} c^{2}} \sum_{j=1}^{N} \vec{A}^{2}\left(\vec{x}_{j}\right)=$

$=N \ln \left(\Delta V\left(\frac{\pi}{\alpha}\right)^{3 / 2}\right)+\frac{3 N}{2}=$

$=-\frac{3 N}{2} \ln \alpha+N \ln \left(\Delta V \pi^{3 / 2}\right)+\frac{3 N}{2}=$

$=-\frac{3 N}{2} \ln \left(\frac{3}{2 \Delta J^{2}}\left(1-\frac{2}{3 N \tau}\left(\Phi+\Phi_{\text {int }}\right)\right)\right)+N \ln \left(\Delta V \pi^{3 / 2}\right)+\frac{3 N}{2}=$

$=\frac{3 N}{2}+N \ln \left(\Delta V\left(\frac{2 \pi \overline{\Delta J^{2}}}{3}\right)^{3 / 2}\right)-\frac{3 N}{2} \ln \left(1-\frac{2}{3 N \tau}\left(\Phi+\Phi_{\text {int }}\right)\right)$.

Another quantity of interest is the free energy defined by $F=\tau \ln P_{0}$. Combining (3.6) with (3.4) and (3.7b,d), we obtain $F=\tau \ln P_{0}=\tau \sum_{j=1}^{N} C_{j}-N \tau \ln \left(\Delta V \pi^{3 / 2}\right)+\frac{3 N \tau}{2} \ln \alpha=$

$=\Phi+\Phi_{\mathrm{int}}-N \tau \ln \left(\Delta V\left(\frac{2 \pi \overline{\Delta J^{2}}}{3}\right)^{3 / 2}\right)+\frac{3 N \tau}{2} \ln \left(1-\frac{2}{3 N \tau}\left(\Phi+\Phi_{\mathrm{int}}\right)\right)$.

The comparison with (3.10) gives the following relation between $F$ and $S$ :

$F=-\tau S+\Phi+\Phi_{\text {int }}+\frac{3 N \tau}{2}$

\section{The thermodynamic limit}

We shall now pass to the limit $N \rightarrow \infty, V \rightarrow \infty$ with $\Delta V$ fixed, considering also as fixed the volume $\Omega$ where the macroscopic (collective) system is localized. That is to say, the collective configuration described by $\vec{j}(\vec{x})$, localized in the volume $\Omega$, is considered as immersed in a background (a heat bath) of particles freely fluctuating in each $\Delta V_{j}(1 \leq j \leq N)$ covering the volume $V>>\Omega$ (electrically neutral in the average). In this limit the entropy and the free energy will be separated in two parts corresponding respectively to the collective system and to the background. The two systems however 
are not independent but they can eventually interact by exchanging energy through the interaction energy $\Phi_{\text {int }}$.

Effects related to the macroscopic configuration and to the fluctuating background are both contained in $\overline{\Delta J^{2}}$, which can be split as follows:

$\overline{\Delta J^{2}}=\frac{1}{N} \int_{\Gamma} P \sum_{j=1}^{N}\left(\vec{j}_{j}\left(\vec{x}_{j}\right)+\overrightarrow{\tilde{j}}_{j}\right)^{2} d \Gamma=$

$=\frac{1}{N} \int_{\Gamma} P \sum_{j=1}^{N}\left(\vec{j}^{2}\left(\vec{x}_{j}\right)+2 \vec{j}\left(\vec{x}_{j}\right) \cdot \overrightarrow{\widetilde{j}}_{j}+\overrightarrow{\tilde{j}}_{j}^{2}\right) d \Gamma=$

$=\frac{1}{N} \sum_{j=1}^{N} \vec{j}^{2}\left(\vec{x}_{j}\right)+\frac{2}{N} \sum_{j=1}^{N} \vec{j}\left(\vec{x}_{j}\right) \cdot \overline{\vec{\jmath}}_{j}+\frac{1}{N} \sum_{j=1}^{N} \overline{\widetilde{j}}_{j}^{2}$

Here the first term is a purely collective part. The second term describes a spatial correlation between the collective current density and the average of the fluctuating current density of the background in interaction with the collective system, and the last term is the mean square of the fluctuations of the background.

Using (3.7a), (3.7c) and (3.7d) and replacing the sums $\sum_{j} \Delta V_{j}$ with integrals $\int_{\Omega} d \Omega$, the last two terms can be written in the form:

$$
\begin{aligned}
& \overline{\overrightarrow{\vec{j}}^{2}} \equiv \frac{1}{N} \sum_{j=1}^{N} \overline{\overrightarrow{\vec{j}}_{j}^{2}}=\frac{3}{2 \alpha}+\frac{1}{V} \int_{\Omega}\left(\vec{j}(\vec{x})+\frac{\Delta V}{2 \alpha \tau c} \vec{A}(\vec{x})\right)^{2} d \Omega, \\
& \Delta J_{c} \equiv \frac{2}{N} \sum_{j=1}^{N} \vec{j}\left(\vec{x}_{j}\right) \cdot \overline{\overrightarrow{\vec{j}}_{j}}=-\frac{2}{V}\left(\int_{\Omega} \vec{j}^{2}(\vec{x}) d \Omega+\frac{\Delta V}{2 \alpha \tau c} \int_{\Omega} \vec{j}(\vec{x}) \cdot \vec{A}(\vec{x}) d \Omega\right) .
\end{aligned}
$$

After inserting (4.2) and (4.3) into (4.1), we obtain

$$
\overline{\Delta J^{2}}=\overline{\vec{j}^{2}}\left(1-\frac{1}{V \overline{\vec{j}^{2}}}\left(\int_{\Omega} \vec{j}^{2}\left(\vec{x}_{j}\right) d \Omega+\frac{\Delta V}{\alpha \tau c} \int_{\Omega} \vec{j}(\vec{x}) \cdot \vec{A}(\vec{x}) d \Omega\right)\right)
$$

In the limit $V \rightarrow \infty$, the last term in (4.4) is much smaller than unity and $\ln \overline{\Delta J^{2}}$ in (3.10) and (3.11) can be approximated by an expansion up to first order in $\Omega / V$. Moreover, the limit $V \rightarrow \infty$ implies the limit $N \rightarrow \infty$ in order that $\Delta V$ remains finite. Since also $\tau$ is finite (see the explicit expression for $\tau$ given by equation (23.18)), it follows that $\left(\Phi+\Phi_{\text {int }}\right) / N \tau$ is much less than unity, so that the last term of (3.10) and (3.11) can also be approximated by expanding the logarithm up to first order in $\left(\Phi+\Phi_{\text {int }}\right) / N \tau$. Finally, recalling (4.2) and that $\Omega<<$, one has $\alpha=3 / 2 \overline{\overrightarrow{\tilde{j}}^{2}}$. It follows that $S$ (3.10) can be expressed as the sum of two parts

$S=S_{b}+S_{p}$,

where $S_{b}$ contains the effects of the background,

$S_{b}=\frac{3 N}{2}+N \ln \left(\Delta V\left(\frac{2 \pi \overline{\vec{j}}^{2}}{3}\right)^{3 / 2}\right)+\frac{\Phi_{\text {int }}}{\tau}$,

and $S_{p}$ depends on the collective quantities of the macroscopic configuration:

$$
S_{p}=-\frac{3}{2 \Delta V \overline{\overrightarrow{\widetilde{j}}^{2}}} \int_{\Omega} \vec{j}^{2}(\vec{x}) d \Omega-\frac{\Phi}{\tau}=
$$


$=-\frac{3}{2 \Delta V \overline{\overrightarrow{\tilde{j}}^{2}}}\left(\int_{\Omega} \vec{j}^{2}(\vec{x}) d \Omega+\frac{\Delta V \overline{\overrightarrow{\widetilde{j}}^{2}}}{3 \pi c} \int_{\Omega} \vec{j}(\vec{x}) \cdot \vec{A}(\vec{x}) d \Omega\right)$.

The free energy is calculated from (3.12), using (4.5) and (4.6):

$F=-N \tau \ln \left(\Delta V\left(\frac{2 \pi \overline{\overrightarrow{\tilde{j}}^{2}}}{3}\right)^{3 / 2}\right)-\tau S_{p}+\Phi$.

Here, for reasons of completeness of the calculation, we have retained terms proportional to $N$. These terms are in fact meaningless as they tend to infinity with $N$ and play the role of infinite constants, independent of the collective equilibrium, while only variations of $S$ and $F$ with respect to collective quantities are physically relevant.

The thermodynamic limits of other quantities of interest are as follows (use is made of (3.7c), (3.7d) and (4.4))

$$
\begin{aligned}
& \frac{1}{N} \sum_{j=1}^{N}\left({\overline{\vec{J}_{j}^{2}}}^{-\vec{J}_{j}^{2}}\right)=\frac{3}{2 \alpha}=\overline{\Delta J^{2}}=\overline{\vec{j}^{2}}, \\
& \tau=-\frac{\Delta V \widetilde{j}^{2}}{6 c^{2}} \frac{\vec{A}^{2}(\vec{x}) d \Omega}{\Phi+\Phi_{\text {int }}}, \\
& \Delta J_{c}=-\frac{2}{V}\left(\int_{\Omega} \vec{j}^{2}(\vec{x}) d \Omega+\frac{\Delta V \overline{\tilde{j}^{2}}}{3 \tau} \int_{\Omega} \vec{j}(\vec{x}) \cdot \vec{A}(\vec{x}) d \Omega\right) .
\end{aligned}
$$

\section{The magnetic entropy concept}

Comparing the expression (4.11) with (4.7), one can write $S_{p}=\frac{3 N}{4 \overrightarrow{\widetilde{j}}^{2}} \Delta J_{c}$.

This relation tells us that the entropy of the macroscopic system is proportional to the correlation in space between the macroscopic current density and the fluctuating current density of the background described by (4.3) and this correlation vanishes for $S_{p}=0$. Let us put $\Phi_{\text {int }}=0$, so that also the correlation in space between the fluctuating current of the background and the collective potential vanishes. After substituting $\tau$ (4.10) into (4.7) we obtain

$$
S_{p}=\frac{3}{2 \Delta V \overline{\vec{j}^{2}} \int_{\Omega} \vec{A}^{2} d \Omega}\left(-\int_{\Omega} \vec{j}^{2} d \Omega \int_{\Omega} \vec{A}^{2} d \Omega+\left(\int_{\Omega} \vec{j} \cdot \vec{A} d \Omega\right)^{2}\right) .
$$

Applying the Schwartz inequality, we find that $S_{p} \leq 0$, Thus, the absolute maximum $S_{p}=0$ is associated with complete absence of correlation between the macroscopic system and the background. This occurs when $\vec{j}=\kappa \vec{A}$ where $\kappa$ is a constant.

By fixing $\Phi_{\text {int }}=0$ and by eliminating $\tau$ from (4.7) we have implicitly supposed that $\tau$ is varying when the macroscopic quantities are varied. A different physical situation arises when we keep $\tau$ fixed and eventually vary $\Phi_{\text {int }}$ instead. Indeed $\tau$ is a kind of generalized temperature (as can be formally seen by inspecting the formulas above) and in the limit of the Maxwellian plasma is related to the 
Maxwellian temperature by a linear relation given in section 23 where its relation with the underlying dynamics of the particles will be discussed. Variations with $\tau$ fixed are therefore isothermal variations. We then consider $\tau$ as a parameter of the macroscopic equilibrium and in view of the comparisons with practical systems we find it convenient to replace $\tau$ with a parameter $\mu$ according to the relation $\tau=-\frac{4 \pi \Delta V \overline{\overline{\vec{j}^{2}}}}{3 \mu^{2} c^{2}}$.

Using this relation, $S_{p}$ can be written in the form

$$
S_{p}=\frac{2 \pi}{\left|\tau \mu^{2}\right| c^{2}}\left(-\int_{\Omega} \vec{j}^{2} d \Omega+\frac{\mu^{2} c}{4 \pi} \int_{\Omega} \vec{j} \cdot \vec{A} d \Omega\right),
$$

and the interaction energy is given by the expression

$$
\Phi_{\mathrm{int}}=-\Phi+\frac{\mu^{2}}{8 \pi} \int_{\Omega} \vec{A}^{2} d \Omega=-\frac{1}{2 c} \int_{\Omega} \vec{j} \cdot \vec{A} d \Omega+\frac{\mu^{2}}{8 \pi} \int_{\Omega} \vec{A}^{2} d \Omega .
$$

A variation of the macroscopic quantities $\vec{j}$ and $\vec{A}$ with $\mu$ (and $\tau$ ) fixed, implies eventually a variation of $\Phi_{\text {int }}$ and, at the same time, the presence of correlation between the fluctuating and the macroscopic quantities and an energy exchange between the collective system and the background. The variation of $\Phi_{\text {int }}$ will be calculated explicitly for typical situations of practical interest in section 9, in section 12 and in section 16 . It will turn out that $\Phi_{\text {int }}$ describes the exchange of energy of the macroscopic system with the external world. Thus, the background allows for simulating the behavior of this system when it is not isolated

\section{Time evolution of the magnetic entropy}

We now consider that the collective equilibrium is time dependent, but changing so slowly as to justify the application in a significant time interval of the concepts of canonical ensemble and canonical average implicit in our procedure.

In this section we shall investigate how the magnetic entropy changes in time when the plasma evolves in accordance with Maxwell equations. For this purpose it should be noted that in the derivation of sections 4-6 the localization of the plasma equilibrium in a finite volume $\Omega$ played an essential role. Thus, in order to proceed consistently with our statistical model we have to express this situation in physical terms. We shall consider a situation where the plasma is carrying a volume current with density $\vec{j}_{p}$ and is in contact with a surrounding conductive shell. The presence of a closed shell has a precise thermodynamic meaning because it allows us to consider the enclosed system as being isolated from the external world. However if narrow cuts exists in the shell which allow the penetration of an electromagnetic field, then the plasma interacts with the external world and the shell defines merely the localization of the plasma equilibrium in the finite volume delimited by the shell. This model will be useful for the discussion of the physical meaning of the interaction energy $\Phi_{\text {int }}$ in section 8.

We assume that a current $\vec{j}_{s}$ exists on the shell, which creates outside the shell a magnetic field screening the magnetic field $\vec{B}$ created by $\vec{j}_{p}$, so that the total magnetic field vanishes outside the shell: $\frac{4 \pi}{c} \vec{j}_{p}=\nabla \times \vec{B}, \quad \frac{4 \pi}{c} \vec{j}_{s}=-\delta\left(\rho-\rho_{s}\right) \vec{e}_{n} \times \vec{B}$. 
The function $\delta\left(\rho-\rho_{s}\right)$, where $\rho$ is the coordinate along the minor radius, describes the localization of $\vec{j}_{s}$ on the conductive shell with effective radius $\rho_{s}$, and, whenever convenient, will be approximated by a $\delta$-function; $\vec{e}_{n}$ is the unit vector orthogonal to the shell and directed outwards.

The entropy functional (5.4) is expressed in terms of the total current density $\vec{j}=\vec{j}_{p}+\vec{j}_{s}$ and $\vec{A}_{p}$, where $\nabla \times \nabla \times \vec{A}_{p}=(4 \pi / c) \vec{j}_{p}$. Thus the collective part $S_{p}$ of the magnetic entropy can be subdivided into a part depending on the plasma quantities $\vec{j}_{p}$ and $\vec{A}_{p}$, denoted again with $S_{p}$, and a part $S_{s}$ which depends only on the current density $\vec{j}_{s}$ on the conductive shell:

$$
\begin{aligned}
& S_{p}=\frac{2 \pi}{\left|\tau \mu^{2}\right| c^{2}}\left(-\int_{\Omega}\left(j_{p}^{2}+2 \vec{j}_{p} \cdot \vec{j}_{s}\right) d \Omega+\frac{\mu^{2} c}{4 \pi} \int_{\Omega}\left(\vec{j}_{p}+\vec{j}_{s}\right) \cdot \vec{A}_{p} d \Omega\right), \\
& S_{s}=-\frac{2 \pi}{\left|\tau \mu^{2}\right| c^{2}} \int_{\Omega} j_{s}^{2} d \Omega=-\frac{1}{\left|\tau \mu^{2}\right| 8 \pi} \int_{\Omega} \delta^{2}\left(\rho-\rho_{s}\right) \vec{B}^{2} d \Omega .
\end{aligned}
$$

where $d \Omega=d \rho d S$ and the integration includes the shell.

We now concentrate on the part $S_{p}$ pertaining to the plasma. Approximating (6.1) with the $\delta$ - function one obtains

$$
S_{p}=\frac{1}{\left|\tau \mu^{2}\right| 8 \pi}\left(\left(-\int_{\Omega}(\nabla \times \vec{B})^{2} d \Omega+2 \int_{\Omega \Omega} \nabla \times \vec{B} \cdot d \vec{S} \times \vec{B}+\mu^{2} \int_{\Omega} \nabla \times \vec{B} \cdot \vec{A}_{p} d \Omega-\mu^{2} \int_{\Omega} d \vec{S} \times \vec{B} \cdot \vec{A}_{p}\right)\right) .
$$

Applying Faraday's law

$\frac{1}{c} \frac{\vec{B}}{\partial t}=-\nabla \times \vec{E}, \frac{1}{c} \frac{\partial \vec{A}_{p}}{\partial}=-\vec{E}$,

and after repeated application of Gauss's theorem, the time derivative of $S_{p}$ takes the following form:

$$
\begin{aligned}
& \frac{d S_{p}}{d t}=\frac{1}{\tau}\left(\int_{\Omega} \frac{\vec{E}}{\mu^{2}} \cdot\left(\nabla^{2} \vec{j}_{p}+\mu^{2} \vec{j}_{p}\right) d \Omega-\frac{1}{\mu^{2}} \int_{\partial \Omega} \vec{E} \times \nabla \times \vec{j}_{p} \cdot d \vec{S}+\frac{c}{4 \pi} \int_{\partial \Omega} d \vec{S} \cdot \vec{E} \times \vec{B}-\frac{1}{c \mu^{2}} \int_{\alpha \Omega} d \vec{S} \cdot \vec{B} \times \vec{\sigma}_{p} / \partial\right)= \\
= & -\frac{1}{\tau}\left(\int_{\Omega}\left(\frac{1}{\mu^{2}} \nabla \times \vec{j}_{p}-\frac{c}{4 \pi} \vec{B}\right) \cdot(\nabla \times \vec{E}) d \Omega\right)-\frac{1}{\tau \mu^{2} C} \int_{\partial \Omega} d \vec{S} \cdot \vec{B} \times \frac{\vec{\sigma}_{p}}{\partial t}
\end{aligned}
$$

where $d \vec{S}=\vec{e}_{n} d S, \nabla \cdot \vec{j}_{p}=0$.

We distinguish two cases:

Case 1: $\vec{E}=\vec{E}_{e x t}=E_{e x t} \vec{e}_{\phi}$ is a toroidal inductive electric field that has penetrated through narrow cuts in the volume enclosed by the copper shell where $\nabla \times \vec{E}_{e x t}=0$, while the magnetic configuration of the plasma is time independent. This is the case of the tokamak in static equilibrium.

Case 2: The plasma is enclosed in a perfectly conductive shell without cuts so that the component of the electric field tangent to the shell vanishes and $\vec{E} \times d \vec{S}=0$ on the shell. In general $\nabla \times \vec{E} \neq 0$ and the magnetic configuration is not static due to dissipation and/or instabilities. With $\vec{\sigma}_{p} / \partial=0$ on $\partial \Omega$ (see section 10) the first expression (6.5) becomes

$$
\frac{d S_{p}}{d t}=\frac{1}{\tau} \int_{\Omega} \frac{\vec{E}}{\mu^{2}} \cdot\left(\nabla^{2} \vec{j}_{p}+\mu^{2} \vec{j}_{p}\right) d \Omega \neq 0 .
$$

The same expression for $d S_{p} / d t$, but with $d S_{p} / d t=0$, is obtained in case 1 when one requires that the volume and surface contributions of the first expression (6.5) for $d S_{p} / d t$ are vanishing separately. This requirement follows from the condition that in the confinement region, under conditions of static equilibrium, the time derivative of $S_{p}$ should vanish locally, that is to say, in every small volume 
element $\Delta \Omega$ of $\Omega$. Indeed, a prerequisite for applying this condition is that the volume and the surface integrals vanish separately. More generally, introducing an arbitrary function $F$ (to be determined later) the volume and the surface contributions can be separated by putting

$$
\begin{aligned}
& \int_{\Omega} \frac{\vec{E}_{e x t}}{\mu^{2}} \cdot\left(\nabla^{2} \vec{j}_{p}+\mu^{2} \vec{j}_{p}\right) d \Omega=-\int_{\Omega} F d \Omega, \\
& \int_{\Omega} F d \Omega=-\frac{1}{\mu^{2}} \int_{\partial \Omega} \vec{E}_{e x t} \times \nabla \times \vec{j}_{p} \cdot d \vec{S}+\frac{c}{4 \pi} \int_{\partial \Omega} d \vec{S} \cdot \vec{E}_{e x t} \times \vec{B}-\frac{1}{c \mu^{2}} \int_{\partial \Omega} d \vec{S} \cdot \vec{B} \times \frac{\vec{\partial}_{p}}{\partial t},
\end{aligned}
$$

where one equality is a consequence of the other.

The requirement that $d S_{p} / d t$ vanishes locally in the tokamak leads to the equation

$\frac{E_{e x t}}{\mu^{2}} \nabla^{2} j_{p}+\mu^{2} j_{p}=-F$,

where $\vec{j}_{p}=j_{\phi} \vec{e}_{\phi}, \vec{E}_{e x t}=E_{e x t} \vec{e}_{\phi}$ and $F$ will be determined in the next section.

\section{CHAPTER 2: Plasma states and state transformations}

Summary: Time independent magnetic configurations of the plasma associated with a timeindependent magnetic entropy are called “plasma states'. The electric field present in a plasma state can only be the stationary electric field induced externally.

The requirement that the magnetic entropy is stationary in the tokamak implies the balance between the net power deposited on electrons and the power lost by electron thermal conductivity. The additional requirement that the balance holds locally leads to an equation for the profile of the current density induced by the external electric field (the SME equation).

One can consider reversible transformations of the states described by the SME equation associated with emission or absorption of electromagnetic energy described by the variation of $\Phi_{\text {int }}$ related to the Poynting flux of energy. The corresponding change in time of the collective magnetic quantities gives rise to an internal inductive electric field and to an increase or decrease of magnetic entropy whose correspondence with the magnetic states is then defined by the reversible process. In the absence of external drive the same states of entropy can be accessed only through an irreversible entropy increase. In section 9 the transformations among states satisfying the SME equations are discussed in detail.

In the absence of external interactions, i.e. when the plasma is completely isolated from the external world by a perfectly conductive shell, the state of maximum entropy is a force-free state. The stability properties of the reversed field pinch are contained in the variation properties of the magnetic entropy and of its time derivative.

\section{Stationary magnetic entropy (SME) and power balance of the tokamak}

As is customary in statistical thermodynamics the physical meaning of the terms contributing to the entropy change emerges after comparison with the power balance equation. For the purpose of this comparison let us write the first equation (6.7) in the form

$$
\frac{d S_{p}}{d t}=\frac{1}{\tau} \int_{\Omega} \frac{\vec{E}_{e x t}}{\mu^{2}} \cdot\left(\nabla^{2} \vec{j}_{p}+\mu^{2} \vec{j}_{p}\right) d \Omega+\frac{1}{\tau} \int_{\Omega} F d \Omega=0 .
$$


Here the second term describes the work of the external electric field on the plasma current density $\vec{j}_{p}=j_{\phi} \vec{e}_{\phi}$, which can be interpreted naturally as the induced current carried by the electrons. Correspondingly, the magnetic entropy calculated with this current density should pertain to the system of electrons.

In conditions of stationary equilibrium the thermal energy of the electrons is constant in time and the power balance is expressed by the relation

$-\int_{\Omega} \vec{q}_{h} \cdot d \vec{S}+\int_{\Omega} \vec{E}_{\text {ext }} \cdot \vec{j}_{p} d \Omega+\int_{\Omega} p_{E} d \Omega=0$.

Here $q_{h}$ is the heat flux related to the thermal conductivity of the electrons and the third term contains the contribution of the net power density deposited on electrons (auxiliary power, electron-ion energy transfer, non diffusive losses) in addition to the Ohmic contribution described by the second term. Thus, according to (7.2), the open system of electrons is in thermal equilibrium with the external ambience. There is neither energy gain per unit time of the electrons, nor global entropy production, the entropy production due to the thermal dissipation in the volume $\Omega$ of the plasma being compensated by the entropy per unit time injected externally into this volume. This is consistent with the relation $d S_{p} / d t=0$ implied in the derivation of (7.1). The comparison between (7.1) and (7.2) leads to the identifications $F=p_{E}$, and

$-\int_{\infty \Omega} \vec{q}_{h} \cdot d \vec{S}=\frac{1}{\mu^{2}} \int_{\Omega} \vec{E}_{e x t} \cdot \nabla^{2} \vec{j}_{p} d V=\frac{1}{\mu^{2}} \int_{\infty \Omega}\left(E_{e x t} \nabla j_{\phi}-j_{\phi} \nabla E_{e x t}\right) \cdot d \vec{S}$.

It is of special interest to study the current density profiles associated with the magnetic entropy that is stationary locally, that is to say, in any arbitrary small toroidal shell $\Delta \Omega$. As follows from (7.1) these profiles are described in the cylindrical limit, by the following equation:

$\nabla^{2} j_{\phi}+\mu^{2} j_{\phi}=-\frac{\mu^{2}}{E_{e x t}} p_{E}$.

In the cylindrical limit the heat flux (7.3) can be expressed in terms of the ratio $s_{h}(r) / q(r)$ between the magnetic shear $s_{h}=(r / q) d q / d r$ and the safety factor. Indeed combining the relation

$$
j(r)=\frac{c}{4 \pi r} \frac{d}{d r}\left(r B_{\theta}\right)=\frac{c B}{4 \pi R r} \frac{d}{d r}\left(\frac{r^{2}}{q(r)}\right),
$$

with the expression (6.3), one obtains

$$
q_{h} S=\frac{c B U}{\mu^{2} R}\left(\frac{s_{h}}{q}+\frac{1}{2} \frac{d}{d r}\left(\frac{s_{h}}{q}\right)+\text { const }\right),
$$

where $U=2 \pi R E_{\text {ext }}$ is the loop voltage, $S=4 \pi^{2} r R$ and the constant is fixed by the boundary condition at the inner border of the confinement region. The heat flux is then expressed in terms of the magnetic configuration, which in turn depends on the power deposited on electrons through the equation (7.4). In order to determine the temperature one needs a constitutive relation, for instance

$-n_{e} \chi_{\text {eff }} \frac{d T_{e}}{d r}=q_{h}$.

The electron temperature $T_{e}$, calculated from eq.(7.7), is therefore the result of the combined effect of the magnetic configuration which follows from the condition (7.4) and of the specific mechanism of thermal transport determining $\chi_{\text {eff }}$, which is not contained in this condition. Further conditions are then needed for fixing at the same time $\chi_{\text {eff }}$ and $T_{e}$ (see the discussion in section 20). 
The validity and the limits of validity of the profiles predicted by the equation (7.4), called the SME (Stationary Magnetic Entropy) equation, will be the object of close scrutiny through comparison with the experimental data (chapter 6). In view of this comparison, it is worthwhile to stress already now the theoretical conditions under which equation (7.4) holds.

It is implicit in the derivation that the electric field $E_{e x t}$ is the external electric field of the ohmic transformer and not the electric field generated eventually inside the plasma. The term $\vec{E}_{\text {ext }} \cdot \vec{j}$ describes the work of the transformer on the induced current sustained by the electrons. The equation is derived assuming that the entropy functional (5.4) is stationary in time and that the plasma is uniformly quiescent everywhere. So the equation holds under completely stationary conditions and dynamical effects (i. e. sawteeth and others) should be excluded. Consistently with eq. (7.2), the integral of the SME equation implies equilibrium of the power balance of the electrons so that the temperature and the density do not depend on time. We shall discuss in section 17 the special role played by the parameter $\mu \mathrm{s} a$ in tokamaks.

It is worthwhile to recall that in the presence of non-inductive currents the loop voltage and the electric field in eq. (7.4) tend to zero. One sees by inspection that the equation breaks down in this case. So the profile of the non-inductive currents cannot be described by eq. (7.4). This is consistent with the fact that the profiles of the non-inductive currents have a different origin described by their specific generation mechanisms. For instance, the bootstrap current depends, among other things, on the pressure profile, which presupposes a pre-existing well-established equilibrium. The non-inductive currents driven externally are the result of a local interaction, which determines their profiles. These external local dynamical manipulations are not taken into account in the statistical derivation of the entropy functional (5.4) which does not make a distinction "a priori" from one plasma region and another, and consequently cannot be contained in eq. (7.4).

The macroscopic current density will be taken as the sum of an inductive part $\vec{j}$, described by equation (7.4), and a non inductive part (assuming that the non inductive part is small and neglecting a possible interaction between the two parts):

$\vec{j}_{\text {tot }}(r)=\vec{j}(r)+\vec{j}_{\text {nind }}(r)$.

The form of $\vec{j}_{\text {nind }}(r)$ is taken from experiment, that is to say, it is calculated from the generation mechanism consistently with the experimental data.

Clearly the validity of the current density profiles described by the equation (7.4) depends on how large are the effects whose description is not included in this equation. For instance, the larger is the non-inductive current or the larger is the sawtooth zone, the larger is the deviation of the profile of the total current density from that described by eq. (7.4). We shall return on these points in the discussion of the experimental data in chapter 6 .

\section{The physical meaning of the interaction energy $\Phi_{\text {int }}$}

An insight into the physical aspects of the statistical model is obtained by inspecting the time derivative $\dot{\Phi}_{\text {int }}$ of the interaction energy $\Phi_{\text {int }}$. Putting $\vec{j}=\vec{j}_{p}+\vec{j}_{s}$ into eq. (5.5) one has

$\Phi_{\mathrm{int}}=-\frac{1}{2 c} \int_{\Omega} \vec{j}_{p} \cdot \vec{A}_{p} d \Omega+\frac{1}{8 \pi} \int_{\alpha \Omega} d \vec{S} \times \vec{B} \cdot \vec{A}_{p}+\frac{\mu^{2}}{8 \pi} \int_{\Omega} A_{p}^{2} d \Omega$, 


$$
\begin{aligned}
& \frac{d \Phi_{\text {int }}}{d t}=-\frac{1}{8 \pi} \int_{\Omega} \vec{A}_{p} \cdot \nabla \times \frac{\partial \vec{B}}{\partial} d \Omega+\frac{1}{2} \int_{\Omega} \vec{j}_{p} \cdot \vec{E} d \Omega+\frac{1}{8 \pi} \int_{\partial \Omega} \vec{A}_{p} \cdot d \vec{S} \times \partial \vec{B} / \partial+ \\
& +\frac{c}{8 \pi} \int_{\partial \Omega} d \vec{S} \cdot \vec{E} \times \vec{B}-\frac{\mu^{2} c}{4 \pi} \int_{\Omega} \vec{A}_{p} \cdot \vec{E} d \Omega
\end{aligned}
$$

Note that the screening field created by $\vec{j}_{s}$ exists only outside $\Omega$ and the same holds for the corresponding vector potential $\vec{A}_{s}$.

After application of Gauss's theorem one is led to the following expression:

$$
\frac{d \Phi_{\text {int }}}{d t}=\frac{c}{4 \pi} \int_{\alpha \Omega} d \vec{S} \cdot \vec{E} \times \vec{B}+\int_{\Omega}\left(\vec{j}_{p}-\frac{\mu^{2} c}{4 \pi} \vec{A}_{p}\right) \cdot \vec{E} d \Omega .
$$

The first term in the right hand side is the Poynting flux of electromagnetic energy across the boundary $\partial \Omega$. To see the meaning of the last term we specialize to the tokamak. We note a relation between $j_{\phi}$ and the related component $A_{\phi}$ of the vector potential, which follows straightforwardly combining (7.4) with D' Alembert equation $\nabla^{2} A_{\phi}=-(4 \pi / c) j_{\phi}$. One obtains

$$
j_{\phi}=\frac{c \mu^{2}}{4 \pi} A_{\phi}+\varphi \text {, where } \nabla^{2} \varphi=-\mu^{2} \frac{p_{E}}{E_{\text {ext }}} .
$$

The term $\varphi$ describes the part of the current density which is generated by the interaction of the plasma with the external world, namely with the power input and output described by $p_{E}$.

Applying (8.4) the last term of (8.3) becomes $\int_{\Omega} \varphi E_{e x t} d \Omega$, which describes the work of the electric field on that part of the current density, which is generated by the net power deposition on the collective system of electrons. It will be shown in section 17 that this term becomes negligible under the conditions that insure the profile consistency in the tokamak when the magnetic entropy is stationary. Thus, under these conditions (to be presented in chapter 4), only the electromagnetic effects described by the Poynting term are present in the expression of $d \Phi_{\text {int }} / d t$ and $d \Phi_{\text {int }} / d t=0$ when the collective system is isolated.

In the case of a force free equilibrium, which satisfies the equations

$\vec{j}=\frac{\mu C}{4 \pi} \vec{B}=\frac{\mu c}{4 \pi} \nabla \times \vec{A}_{p}=\frac{c}{4 \pi} \nabla \times \vec{B}$.

The integrand of the last term of (8.3) can also be seen to vanish after a proper choice of the gauge. Indeed, if the equations above are satisfied by $\vec{A}_{p}$ they are also satisfied by $\vec{A}_{p}+\nabla \varphi$. Let us determine $\varphi$ from the equation

$\frac{\mu c}{4 \pi} \vec{A}_{p}+\nabla \varphi=\frac{c}{4 \pi} \vec{B}=\frac{\vec{j}}{\mu}$.

This equation can be integrated and $\varphi$ is known, the integration constant apart. If then $\vec{A}_{p}$ in (8.3) is replaced by the new gauge $\vec{A}_{p}+(4 \pi / \mu c) \nabla \varphi=\left(4 \pi / \mu^{2} c\right) \vec{j}$, the integrand vanishes identically.

\section{Adiabatic transformations}

Let us consider the infinitesimal transformation $j_{\phi} \rightarrow j_{\phi}+\delta j$ where $\delta j=\left(\mu^{2} c / 4 \pi\right) \delta A$ with $\delta A$ satisfying the equation $\nabla^{2} \delta A+\mu^{2} \delta A=0$. It is immediately seen that $j_{\phi}+\delta j$ satisfies the same equation (7.4) for $j_{\phi}$. It will be shown that the magnetic entropy of the total system formed by the collective 
equilibrium and by the background is an invariant of the transformation above, while the entropy of the two subsystems is varying separately at first order. It follows that no heat can be emitted or absorbed by the total system as a consequence of a reversible transformation, which is therefore adiabatic.

We assume that the total current is conserved during the variation so that the poloidal component of $\overrightarrow{\delta B}$ vanishes on $\partial \Omega$, which gives a condition on the radial derivative of $\delta A_{\varphi}$. The first variation of $S_{p}$ (6.3) is then (dropping the subscripts)

$$
\begin{aligned}
& \delta S_{p}=-\frac{2 \pi}{\tau \mu^{2} c^{2}}\left(-2 \int_{\Omega} j \delta j d \Omega+\frac{\mu^{2} c}{4 \pi} \int_{\Omega}(A \delta j+j \delta A) d \Omega+\frac{c}{4 \pi} \int_{\partial \Omega} \overrightarrow{\delta j} \cdot d \vec{S} \times \vec{B}\right)= \\
& =\frac{2 \pi}{\tau \mu^{2} c^{2}} \int_{\Omega} \delta j\left(j-\frac{\mu^{2} c}{4 \pi} A\right) d \Omega-\frac{1}{2 \tau \mu^{2} c} \int_{\partial \Omega} \overrightarrow{\delta j} \cdot d \vec{S} \times \vec{B} .
\end{aligned}
$$

The first variation of $\Phi_{\text {int }}$ (8.1) is given by the expression

$$
\begin{aligned}
& \delta \Phi_{\mathrm{int}}=-\frac{1}{2 c} \int_{\Omega} \delta A\left(j-\frac{\mu^{2} c}{4 \pi} A\right) d \Omega+\frac{1}{2 \mu^{2} c} \int_{\partial 2} \overrightarrow{\delta j} \cdot d \vec{S} \times \vec{B}= \\
& =-\frac{2 \pi}{\mu^{2} c^{2}} \int_{\Omega} \delta j\left(j-\frac{\mu^{2} c}{4 \pi} A\right) d \Omega+\frac{1}{2 \mu^{2} c} \int_{\partial \Omega} \overrightarrow{\delta j} \cdot d \vec{S} \times \vec{B} .
\end{aligned}
$$

Recalling that $S_{b}=\Phi_{\text {int }} / \tau$ is the entropy pertaining to the background we have that the first variation of the entropy of the total system vanishes

$\delta S=\delta S_{p}+\delta S_{b}=0$.

Let us now consider the second variation of $S$. One has for generic primary variations $\delta A$ and $\delta j=-(c / 4 \pi) \nabla^{2} \delta A$ :

$\delta^{2} S_{p}=\frac{2 \pi}{\tau \mu^{2} c^{2}}\left(\int_{\Omega}(\delta j)^{2} d \Omega-\frac{\mu^{2} c}{4 \pi} \int_{\Omega} \delta j \delta A d \Omega\right)$,
$\delta^{2} S_{b}=\frac{1}{2 \tau c}\left(-\int_{\Omega} \delta j \delta A d \Omega+\frac{\mu^{2} c}{4 \pi} \int_{\Omega}(\delta A)^{2} d \Omega\right)$.

One then obtains the following inequality

$\delta^{2} S=\delta^{2} S_{p}+\delta^{2} S_{b}=\frac{1}{\tau} \int_{\Omega}\left(\frac{2 \delta j}{\mu c}-\frac{\mu \delta A}{2 \pi}\right)^{2} d \Omega \leq 0$,

where the equality holds only for the adiabatic variations that we are considering. It follows from (9.5) that the total system formed the collective equilibrium plus background, considered as isolated, is thermodynamically stable because the entropy $S$ is maximum, the adiabatic variations $\delta j=\left(c \mu^{2} / 4 \pi\right) \delta A$ apart. Stability is a theoretically necessary prerequisite in order that the total system could be identified as a not fading but present object on which conceptual experiments can be performed.

We have seen that the entropy of the total system formed by the collective equilibrium plus background is invariant under the adiabatic variations so that no heat can be emitted or absorbed by the total system in a reversible transformation. The total system is then isolated during the transformation while, in contrast, entropy can be reversibly exchanged at first order between the collective subsystem and the background. The variation of the collective equilibrium is produced by varying the parameters that enter the solution to the equation (7.4) as for instance the location of the inner boundary of the relaxed region (the surface $q=1$ ) and the boundary values of the current density and of the related 
magnetic quantities. At the same time the total current can be kept fixed while electromagnetic energy is emitted or absorbed by external sources described by the variation of $\Phi_{\text {int }}$.

It is instructive to see the rate of change at first order of the entropy and of $\Phi_{\text {int }}$ due to the adiabatic time dependence of the parameters. We neglect at this moment the inhomogeneous term of (7.4). This will be justified in section 17. In this case eq. (8.4) becomes $j=\left(\mu^{2} c / 4 \pi\right) A$ and

$\frac{\partial j}{\partial t}=\frac{\mu^{2} c}{4 \pi} \frac{\partial A}{\partial t}=-\frac{\mu^{2} c^{2}}{4 \pi} E$.

For calculating the first derivative of $S_{p}$ we apply the first expression (6.5). Since the SME equation is preserved in the transformation, the first term in the right hand side of (6.5) vanishes. The second term, using the relations above for $j$, can easily be seen to combine with the third and the last terms to give

$\frac{d S_{p}}{d t}=-\frac{c}{4 \pi \tau} \int_{\partial 2} d \vec{S} \cdot \vec{E} \times \vec{B}$

Thus, recalling (8.3) we have

$\tau \frac{d S_{p}}{d t}=-\frac{c}{4 \pi} \int_{\partial 2} d \vec{S} \cdot \vec{E} \times \vec{B}=-\frac{d \Phi_{\text {int }}}{d t}$.

This relation shows that the electromagnetic energy lost by the background is gained by the collective system (and vice versa) and confirms the interpretation of the interaction energy $\Phi_{\text {int }}$ as a formal device for simulating the interaction of the collective system with external sources of energy. The rate of change in time of the entropy can be expressed in terms of the changing magnetic configuration by the equality

$\tau \frac{d S_{p}}{d t}=\frac{1}{4 \pi} \int_{\Omega} B_{\theta} \frac{\partial B_{\theta}}{\partial t} d \Omega-\frac{1}{c} \int_{\Omega} j \frac{\partial A}{\partial t} d \Omega$.

In the SME states there is a balance between the loss of energy related to the heat flux and the ohmic input in the stationary plasma. The relations (9.8) and (9.9) describe a supplementary input or output of electromagnetic energy associated with the adiabatic change in time of the parameters of the SME states and of the entropy. This exchange of electromagnetic energy with external sources is a reversible process. But the same change of entropy occurs when the transition between the same states is irreversible. The entropy change is then defined also in the realistic case where the infinite background does not exist and the collective system is isolated and undergoes an irreversible transformation. So, albeit fictitious, the introduction of the background allows the definition on ideal theoretical grounds of the entropy changes of the magnetic macroscopic equilibrium through reversible processes involving energy exchanges with external sources.

While in the isolated system the entropy is constant in a reversible transformation, the irreversible change of state described by (9.9) can only occur with $d S_{p} / d t>0$. This establishes a direction in the transitions among the SME states when they can be treated as isolated: only those transitions are allowed such that (9.9) is negative (remember that $\tau$ is negative, see eq. (5.3))).

We will illustrate these points by constructing explicitly the SME states that can be accessed from one another by conserving the total current during the quasi-static change of magnetic entropy related to emission or absorption of electromagnetic energy according to (9.8). Recalling (9.9) the entropy change in the relaxed region, at first order of the varied quantities, is given by the following expression (in mks units): 


$$
|\tau| \Delta S \approx 4 \pi^{2} a^{2} R \cdot 10^{6}\left(-\frac{10}{4 \pi} \int_{\lambda}^{1} B_{\theta} \Delta B_{\theta} x d x+\int_{\lambda}^{1} j \Delta A x d x\right),
$$

where $\tau$ is given by (5.3) and is fixed, $x=r / a$ and $x=1, x=\lambda$ are the outer and inner borders of the relaxed region.

In the sawtooth region, where the theory is not expected to hold, the magnetic configuration is modeled by taking for the safety factor the expression $q(x)=q_{0}+\left(1-q_{0}\right)(r / \lambda)^{2}$. A JET-like geometry is assumed ( $R / a=3, a=1 \mathrm{~m}$ ), but, for simplicity, a circular cross-section for the magnetic surfaces will be assumed here, although a geometrical correction for taking into account the non-circularity will be applied when the theoretical data will be compared with the observations (chapter 6).

We take 1.5MA for the total current and 3.3T for the toroidal field. The location of the $q(\lambda)=1$ surface is taken at $\lambda=0.18$ and the total current is kept fixed. Once the continuity conditions for the current density and for the poloidal field across the $q(\lambda)=1$ surface are satisfied, while the poloidal magnetic flux is left unchanged on this surface, (it turns out that the flux remains practically fixed also on the minor axis) one is left with one parameter free. This is the poloidal flux across the border or, related to it, the value of the current density at the border. In Table I $\Delta \psi<0$ is the outgoing poloidal flux (divided by $2 \pi$ ), $q_{0}$ is the value of the safety factor on the minor axis, $j(\lambda), j(1)$ are the values of the current density at the inner and outer border respectively, and $|\tau| \Delta S_{p}=\Delta \Phi_{\text {int }}$ is the electromagnetic energy released by the collective system when the entropy increases.

Fig. 1 shows the evolution of the current density profiles when the parameters of Table I are varying. One observes that the increase of entropy and the concomitant release of electromagnetic energy are accompanied by outgoing poloidal flux, by the increase of the current density on axis and by a pronounced increase of the current density at the border together with a decrease near the $q=1$ surface.

Table I. Meaning of symbols: $\Delta \psi$, outgoing poloidal flux (divided by $2 \pi$ ); $q_{0}$, safety factor on magnetic axis; $j(\lambda)$, current density on the $q=1$ surface; $j(1)$, current density at the outer border of the relaxed region; $\Delta \Phi_{\text {int }}$, electromagnetic energy released associated with the magnetic entropy increase $\Delta S_{p}$.

\begin{tabular}{lllll}
\hline$\Delta \psi$ & $q_{0}$ & $j(\lambda)$ & $j(1)$ & $|\tau| \Delta S_{p}=\Delta \Phi_{\text {int }}$ \\
$T-m^{2}$ & & $M A / m^{2}$ & $M A / m^{2}$ & $M J$ \\
\hline 0.00 & 0.98 & 1.72 & 0.022 & 0.00 \\
-0.026 & 0.92 & 1.62 & 0.057 & 0.307 \\
-0.053 & 0.87 & 1.52 & 0.088 & 0.614 \\
-0.079 & 0.81 & 1.41 & 0.12 & 0.922 \\
-0.10 & 0.77 & 1.34 & 0.15 & 1.150 \\
\hline
\end{tabular}

When the system can be considered as isolated from the external circuits, the change of entropy is no longer related to the energy output $\Delta \Phi_{\text {int }}$, but the same states of higher entropy described by (8.9) can be accessed by means of an irreversible process where the electromagnetic energy released in the transformation is dissipated internally to the system. 
We note that the transformations in the opposite direction, i.e. in the direction of the decreasing entropy, can only occur when the collective system is not isolated because they imply an input of electromagnetic energy from external sources.

If one admits that the current is ohmically relaxed, the statements on the current density can be transferred to the temperature profile $T \approx j^{2 / 3}$. In particular, the increase of temperature at the border is accompanied by a decrease of temperature near the $q=1$ surface (and vice versa).

Figure 1. Current density of a succession of SME states with constant total current and fixed position of the $q=1$ surface. The direction $0 \rightarrow 4$ (from flat to peaked profile at the centre and increase of the current density at the border) is associated with increase of the entropy $\Delta S_{p}$, release of electromagnetic energy $\Delta \Phi_{\text {int }}$ and outgoing poloidal flux $\Delta \psi$ (see Table I).

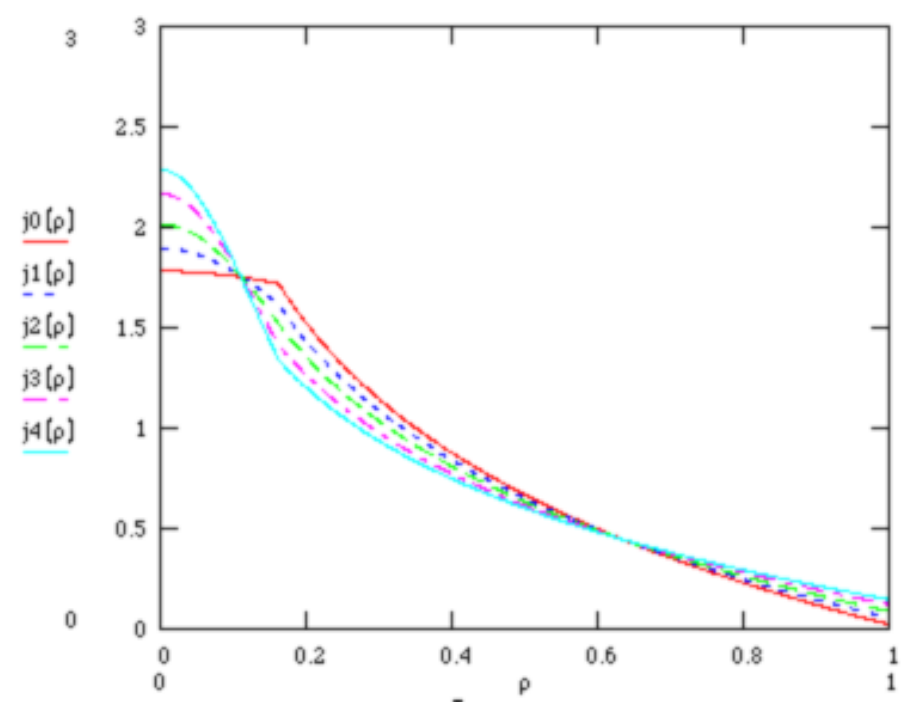

\section{The force-free state}

We now consider the case 2 of section 6, which applies to the plasma isolated from the external world by a perfectly conductive shell so that, in contrast to the tokamak, one has $d \Phi_{\text {int }} / d t=0$. We shall investigate the behavior of the entropy with respect to infinitesimal primary variations $\overrightarrow{\delta j}$, static or quasi-static (i.e. near the marginal point of stability, when it exists) around a static equilibrium, in order to determine the state of maximum entropy. The variations $\delta \vec{B}, \delta \vec{A}$ and $\overrightarrow{\delta j}_{s}$ follow from $\overrightarrow{\delta j}$ according to the equations

$$
\nabla \times \delta \vec{B}=\frac{4 \pi}{c} \overrightarrow{j j}, \quad \nabla \times \delta \vec{A}=\delta \vec{B}, \quad \frac{4 \pi}{c} \vec{j}_{s}=-\delta\left(\rho-\rho_{s}\right) \vec{e}_{n} \times \delta \vec{B} .
$$

Putting $S_{p}=S_{p 0}+\delta S_{p}+\delta^{2} S_{p}$ one has for $\delta S_{p}$ :

$\delta S_{p}=-\frac{1}{4 \pi \tau \mu^{2}} \int_{\partial 2} \delta \vec{B} \cdot\left(\nabla^{2} \vec{B}+\mu^{2} \vec{B}\right) d \Omega+\frac{1}{\tau \mu^{2} C} \int_{\partial \Omega} d \vec{S} \times \overrightarrow{\delta j} \cdot \vec{B}$.

We put $\overrightarrow{\delta j}=0$ at the toroidal wall $\partial \Omega$. To see the physical meaning of this condition we note that it implies $\nabla \times \delta \vec{B}=0$ at the wall, so that one can write $\overrightarrow{B B}=\nabla \chi$ where $\chi$ is some function that we take as a periodic function of the toroidal and poloidal coordinates on the wall. Thus, denoting with $C_{T}$ any 
toroidal path around the wall of the torus, one has that $\oint_{C_{T}} \delta \vec{B} \cdot d \vec{l}=0$ which implies that the current through the hole of the torus is kept constant during the variation [5].

It follows from (10.2) that $S_{p}$ is stationary if the magnetic configuration satisfies the equation $\nabla^{2} \vec{B}+\mu^{2} \vec{B}=\nabla \varphi$,

where $\varphi$ is arbitrary. The force-free state $\nabla \times \vec{B}=\mu \vec{B}$ satisfies this equation with $\nabla \varphi=0$.

The second variation $\delta^{2} S_{p}$ has the form

$\delta^{2} S_{p}=\frac{1}{|\tau| 8 \pi \mu^{2}} \int_{\Omega} \delta \vec{B}\left(\nabla^{2} \delta \vec{B}+\mu^{2} \vec{B}\right) d \Omega$.

In order to discuss the sign of $\delta^{2} S_{p}$ let us consider the eigenvalues $\kappa_{m}$ of the equation $\nabla \times \nabla \times \vec{a}_{m}=\kappa_{m} \nabla \times \vec{a}_{m}$,

with boundary condition $\vec{a}_{m}=0$ [6]. Noting that $\vec{B}_{m}=\vec{a}_{m}+\nabla \psi$ is also a solution of (9.5) and determining $\psi$ in order that $\nabla \cdot \delta \vec{B}_{m}=0$ with $\left(\delta \vec{B}_{m}\right)_{n} \equiv d \vec{S} \cdot \nabla \psi=0$ at the boundary, one has $\nabla^{2} \delta \vec{B}_{m}+\kappa_{m}^{2} \delta \vec{B}_{m}=0$.

Identifying the variation $\overrightarrow{\delta B}$ in (10.4) with a mode $\delta \vec{B}_{m}$ we obtain

$\delta^{2} S_{p}=\frac{1}{|\tau| 8 \pi \mu^{2}} \int_{\Omega} \delta B_{m}^{2}\left(-\kappa_{m}^{2}+\mu^{2}\right) d \Omega$.

It follows that for $\mu^{2}<\kappa_{m}^{2}$ one has $\delta^{2} S_{p}<0$ so that $S_{p}$ is at a maximum. If $\kappa_{m}$ is the lowest eigenvalue of eq. (10.5) the magnetic entropy is maximum with respect to all eigenmodes $\delta \vec{B}_{m}$.

One can make predictions on the time evolution of the perturbed system by inspecting the time dependence of the second variation. The first and the second variation of $d S_{p} / d t$ (6.5) are as follows:

$\delta \frac{d S_{p}}{d t}=\frac{1}{4 \pi|\tau| \mu^{2}} \int_{\Omega} \frac{\partial \delta \vec{B}}{\partial t} \cdot\left(\nabla^{2} \vec{B}+\mu^{2} \vec{B}\right) d \Omega=-\frac{c}{4 \pi|\tau| \mu^{2}} \int_{\Omega}\left(\nabla^{2} \vec{B}+\mu^{2} \vec{B}\right) \cdot \nabla \times \vec{E} d \Omega$,

$\delta^{2} \frac{d S_{p}}{d t}=\frac{1}{4 \pi|\tau| \mu^{2}} \int_{\Omega} \frac{\partial \delta \vec{B}}{\partial t} \cdot\left(\nabla^{2} \delta \vec{B}+\mu^{2} \delta \vec{B}\right) d \Omega$

where we have put $\partial \delta \mathrm{j} / \partial=0$ on $\partial \Omega$.

In order to see how the system evolves in time let us consider the case where $\delta^{2} S_{p}<0$ and $\kappa_{m}^{2}>\mu^{2}$, so that $S_{p}$ is at a maximum. Since the varied system, supposed to be isolated, but time dependent, must evolve to states of increased entropy, one must have $d\left(\delta^{2} S_{p}\right) / d t>0$ and

$\delta^{2} \frac{d S_{p}}{d t}=\frac{1}{8 \pi|\tau| \mu^{2}} \int_{\Omega} \frac{\partial\left(\delta \vec{B}_{m}\right)^{2}}{\partial}\left(-\kappa_{m}^{2}+\mu^{2}\right) d \Omega>0$.

Therefore a sub-domain $\Delta \Omega$ of $\Omega$ exists where $\frac{\partial\left(\delta \vec{B}_{m}\right)^{2}}{\partial}<0$

It follows that $\left|\delta \vec{B}_{m}\right|$ tends to zero in $\Delta \Omega$ with time. Then, after some time, the contribution to the integral (9.10) arises only from the domain outside $\Delta \Omega$. If one admits that $d\left(\delta^{2} S_{p}\right) / d t>0$ in any $\Delta \Omega$, then $\left|\delta \vec{B}_{m}\right|$ must tend to zero everywhere. Conversely one finds that if $S_{p}$ is not at a maximum, so that $\mu^{2}>\kappa_{m}^{2}$, a sub-domain $\Delta \Omega$ exists where $\partial\left|\delta \vec{B}_{m}\right|^{2} / \partial>0$, and the equilibrium is unstable. 
The formulation above of the time evolution of the perturbed force-free state, is based on the same eigenvalue equation (10.5) that determines the stability of the reversed field pinch in the Taylor's model [7]. In this model the magnetic energy is minimized under the constraint of fixed helicity. This

is defined as $K=\int_{\Omega} \vec{A} \cdot \vec{B} d \Omega$ where $\Omega$ is the whole volume of a dissipative plasma. The energy is minimum and the equilibrium is stable when $\mu<\kappa_{m}$ where $\kappa_{m}$ is the lowest eigenvalue of eq.(10.5). Taylor has pointed out the relevance of this mathematical formulation for the description of relaxation and of the magnetic reconnection in plasmas [8]. A derivation of the more general equation (10.3) from a variation functional similar to $S_{p}(5.4)$ has been given in 1958 by Chandrasekhar and Woltjer [9].

In the present formulation the time dependent magnetic energy is compensated by the term of work involving perturbed inductive electric fields and currents generated internally to the collective system. The helicity conservation is not assumed explicitly but it is necessary for restricting the solutions of (10.3) (see Woltjer [10]).

Although the mathematical formulation of the stability of the pinch based on equation (10.5) is the same as that of the Taylor's model, the derivation from the entropy concept is more comprehensive. The entropy concept provides a unifying point of view in the description of the global ideal properties of the tokamak and of the reversed field pinch as well as of effects related to the local structure of the magnetic field, to be discussed in the next paragraphs. The Taylor's model of the pinch was the first look on a new land, ready to be explored.

\section{CHAPTER 3: Local entropy and local plasma properties}

Summary: The plasma states considered until now were global states involving the whole plasma volume and the entropy and its variations were defined with an integral over this volume. However it is often observed that the plasma stability depends on the excitation of localized perturbations or internal modes which give rise to localized structures. It is then worthwhile to investigate the properties of the magnetic entropy with respect to localized variations. In this chapter the entropy functional is expressed in a form sensible to localized perturbations. It turns out that, on one hand, the sign of the second variation of the magnetic entropy depends on the positive or negative definite character of a certain quadratic form, and, on the other hand, that the sign of the quadratic form is related to the stability with respect to interchange modes. In the case of the anisotropic plasma this leads naturally to the magnetic well as a state whose entropy is locally at a maximum.

The quadratic form expresses the energy change arising from interchange of adjacent filaments of current and depends on the physical effects which give rise to the density of current. When the current density introduced in the quadratic form arises from the inhomogeneous magnetic field, the sign of the quadratic form is related to the low- $\beta$ interchange instability.

In situations where the magnetic energy decays as a consequence of localized dissipative effects, as in the case of a dissipative resonant layer, the energy transfer from the magnetic system to the background (or to the external world) is taken into account by the variation of the interaction energy $\Phi_{\text {int }}$. This is the case of the tearing modes to which the present thermodynamic concepts can be instructively applied (section 15). 


\section{Local entropy variations and local structure of the magnetic equilibrium}

In the preceding sections the properties of the magnetic entropy functional where studied with respect to changes of the magnetic configuration involving the whole plasma volume. In order to include in our thermodynamic description also those physical aspects that are localized in a limited region of the plasma and depend on the local structure of the magnetic field one should introduce a definition of the entropy appropriate for this purpose.

A convenient procedure consists in dividing the plasma into relatively small but macroscopic volume elements $\Delta \omega_{n}$ and in calculating the entropy $S_{n}$ in each $\Delta \omega_{n}$ by taking into account the local structure in $\Delta \omega_{n}$ and assuming that the entropy of the global system is the sum of all the $S_{n}$. Of course, $\Delta \omega_{n}$ should be large enough to satisfy $\Delta \omega_{n}>\Delta V=V / N$ as is required by the statistical description. On the other hand, when one looks at the magnetic structure locally inside each $\Delta \omega_{n}$, what really matters is not the average value of the current density or of the vector potential in $\Delta \omega_{n}$, but how these quantities are changing inside $\Delta \omega_{n}$, for instance, with respect to the average value. In other words the information variable consistent with our present point of view to be introduced in the calculation of $\S 1$ of the entropy functional is not $\vec{j}(\vec{x})$ but its deviation $\vec{j}(\vec{x})-\vec{j}\left(\vec{x}_{n}\right)$ from its value at some point $\vec{x}_{n}$ inside $\Delta \omega_{n}$. Correspondingly one should use the deviation $\vec{A}(\vec{x})-\vec{A}\left(\vec{x}_{n}\right)$ instead of $A(\vec{x})$. The calculation that leads to the entropy functional (5.4) and to the interaction energy (5.5) remains unchanged when the information variable $\vec{j}(\vec{x})$ and the vector potential $\vec{A}(\vec{x})$ are replaced everywhere by $\vec{j}(\vec{x})-\vec{j}\left(\vec{x}_{n}\right)$ and $\vec{A}(\vec{x})-\vec{A}\left(\vec{x}_{n}\right)$. Thus, in accordance with our considerations, the entropy of the whole system takes on the form

$$
S_{p}=\sum_{n} S_{p n}=\frac{3}{2 \Delta V \widetilde{\tilde{j}}^{2}} \sum_{n}\left(-\int_{\Delta \omega_{n}}\left(\vec{j}-\vec{j}_{n}\right)^{2} d \omega_{n}+\frac{\mu_{n}^{2} c}{4 \pi} \int_{\Delta \omega_{n}}\left(\vec{j}-\vec{j}_{n}\right) \cdot\left(\vec{A}-\vec{A}_{n}\right) d \omega_{n}\right) \text {, }
$$

where , in accordance with (3.10) $\mu_{n}$ is given by the equality

$\mu_{n}^{2}=-\frac{4 \pi \Delta V \overline{\widetilde{j}^{2}}}{3 c^{2} \tau_{n}}=\frac{4 \pi}{c} \frac{\int\left(\vec{j}-\vec{j}_{n}\right) \cdot\left(\vec{A}-\vec{A}_{n}\right) d \omega_{n}+2 c \Phi_{\text {int }}}{\int_{\Delta \omega_{n}}\left(\vec{A}-\vec{A}_{n}\right)^{2} d \omega_{n}}$.

We start from a zero order equilibrium with $\Phi_{0, \text { int }}=0$ and consider variations of $S_{p}$ and $\Phi_{\text {int }}$ with respect to primary variations of the current density and of the vector potential by keeping $\tau_{n} \propto \mu_{n}^{-2}$ fixed at the equilibrium value (isothermal perturbations). In this situation the sign and the form of the numerator of $\mu_{n}^{2}$ (see eq. (11.2) with $\Phi_{\text {int, }}=0$ ) are crucial for determining the variation properties of $S_{p}$ and, at the same time, the structure of the magnetic equilibrium, as we shall see.

We suppose that the volume elements $\Delta \omega_{n}$ are sufficiently small so that the magnetic field of the equilibrium can be considered as uniform in $\Delta \omega_{n}$ and one can write in first order in $\vec{x}-\vec{x}_{n} \in \Delta \omega_{n}$, $\vec{A}(\vec{x})-\vec{A}\left(\vec{x}_{n}\right)=(1 / 2) \vec{B}\left(\vec{x}_{n}\right) \times\left(\vec{x}-\vec{x}_{n}\right)_{\perp}$ and $\vec{j}(\vec{x})-\vec{j}\left(\vec{x}_{n}\right)=\left(\vec{x}-\vec{x}_{n}\right) \cdot \nabla \vec{j}\left(\vec{x}_{n}\right)$.

Putting $\vec{\xi} \equiv \vec{x}-\vec{x}_{n}$, the equality (10.2) becomes

$\mu_{n}^{2}=\frac{8 \pi}{c} \frac{\int_{\Delta \omega_{n}}(\vec{\xi} \cdot \nabla \vec{j}) \cdot\left(B\left(\vec{x}_{n}\right) \times \vec{\xi}\right) d \omega_{n}}{\int_{\Delta \omega_{n}} B\left(\vec{x}_{n}\right)^{2} \xi_{\perp}^{2} d \omega_{n}}$. 
We shall show the following relation:

$\left(\vec{B} \nabla^{2} \vec{B}\right)_{\vec{x}=\vec{x}_{n}}=-B^{2} \mu_{n}^{2}$.

Let us introduce curvilinear coordinates $\alpha^{i}(i=1,2.3)$ where $\alpha^{1}, \alpha^{2}$ are such to be constant along the magnetic lines of force, with $\vec{B}=\nabla \alpha^{1} \times \nabla \alpha^{2}$, while $\nabla \alpha^{3}$ is parallel to $\vec{B}$

$g^{i k}=\nabla \alpha^{i} \cdot \nabla \alpha^{k}, \quad B^{i}=\vec{B} \cdot \nabla \alpha^{i}, \quad j^{i}=\vec{j} \cdot \nabla \alpha^{i}$.

Noting that $\nabla^{2} \vec{B}=-(4 \pi / c) \nabla \times \vec{j}$, one has in this co-ordinate system that

$\vec{B} \nabla^{2} \vec{B}=\frac{4 \pi}{c} j_{, s}^{i} \varepsilon_{i r k} B^{r} \nabla \alpha^{s} \cdot \nabla \alpha^{k}$,

and

$\mu_{n}^{2}=-\left(\frac{8 \pi}{c}\right) \frac{\int_{\Delta \omega_{n}} j_{, s}^{i} \varepsilon_{i k r} B^{r} d \alpha^{s} d \alpha^{k} d \omega_{n}}{B^{2} \int_{\Delta \omega_{n}} \xi_{\perp n}^{2} d \omega_{n}}=-\left(\frac{8 \pi}{c}\right) \frac{j_{, s}^{i} \varepsilon_{i k r} B^{r} \int_{\Delta \omega_{n}} d \alpha^{s} d \alpha^{k} d \omega_{n}}{B^{2} \int_{\Delta \omega_{n}} \xi_{\perp n}^{2} d \omega_{n}}$,

where $\varepsilon_{i k r}$ is the Ricci tensor ( $\varepsilon_{i k r} \neq 0$ for $i \neq k \neq r$ and equal to $\pm \sqrt{\left|\operatorname{Detg}_{i k}\right|}$ according as an even or odd number of transpositions changes the order of $i, k, r$ to $1,2,3$ where $\varepsilon_{123}>0$ ).

Let us take for $\Delta \omega_{n}$ a volume element with cylindrical cross section and $d \omega_{n}=\rho d \rho d z d \theta$ where $\rho$ and $\vartheta$ are polar coordinates in the plane perpendicular to $\vec{B}$ spanned by the vectors $\nabla \alpha^{1}$ and $\nabla \alpha^{2}$, and $d z$ is parallel to the local direction of $\vec{B}$. The quantity $j_{, s}^{i} \varepsilon_{i k r} B^{r}$ can be taken out of the integral when only terms of second order in $\vec{\xi}$ are taken into account inside $\Delta \omega_{n}$. Denoting with $\vartheta, \vartheta_{s}, \vartheta_{k}$ the polar angles of the vectors $\vec{\xi}_{\perp}, \nabla \alpha^{k}, \nabla \alpha^{s}$ respectively in the perpendicular plane $(s, k=1,2)$ one has, recalling the last equation (11.5)

$\int_{\Delta \omega_{n}} d \alpha^{s} d \alpha^{k} d \omega_{n}=\left|\nabla \alpha^{s} \| \nabla \alpha^{k}\right| \int_{\Delta z} d z \int_{0}^{\bar{\rho}} \xi_{\perp}^{2} \rho d \rho \int_{0}^{2 \pi} d \vartheta \cos \left(\vartheta-\vartheta_{s}\right) \cos \left(\vartheta-\vartheta_{k}\right)$,

where $\left|\nabla \alpha^{s}\right|$ and $\left|\nabla \alpha^{k}\right|$ have been taken out of the integral in view of the assumed uniformity of the equilibrium magnetic field inside $\Delta \omega_{n}$

Applying known formulas of trigonometry (11.8) becomes

$\int_{\Delta \omega_{n}} d \alpha^{s} d \alpha^{k} d \omega_{n}=\left|\nabla \alpha^{s}\right| \nabla \alpha^{k} \mid \pi \int_{\Delta z} d z \int_{0}^{\bar{\rho}} \rho \xi_{\perp}^{2} d \rho \cos \left(\vartheta_{k}-\vartheta_{s}\right)=\frac{1}{2} \nabla \alpha^{s} \cdot \nabla \alpha^{k} \int_{\Delta \omega_{n}} \xi_{\perp}^{2} d \omega_{n}$.

For the integrals involving the third component we have

$\int_{\Delta \omega_{n}} d \alpha^{3} d \alpha^{k} d \omega_{n}=\left|\nabla \alpha^{3}\right|\left|\nabla \alpha^{k}\right| \int_{\Delta \omega_{n}}\left|\xi_{\|}\right| \rho d \rho \int_{0}^{2 \pi} \cos \left(\vartheta-\vartheta_{k}\right) d \vartheta=0$,

where, in view of the properties of the Ricci tensor, one must have $k \neq 3$.

The relation (11.4) is then obtained combining (11.9), (11.7) and (11.6). We note that the parallel component of $\vec{\xi}$ is irrelevant in view of (11.10).

Through the relation (11.4) one can connect $\mu_{n}^{2}$ with the pressure tensor $\vec{P}$ of the equilibrium, a fact that will be useful in the following. Indeed,by applying the equation of the equilibrium,

$\vec{j} \times \vec{B}=c \nabla \cdot \vec{P}$

to the relation

$\vec{B} \nabla^{2} \vec{B}=-\frac{4 \pi}{c} \vec{B} \cdot \nabla \times \vec{j}=-\frac{4 \pi}{c}(\vec{j} \cdot \nabla \times \vec{B}+\nabla \cdot \vec{j} \times \vec{B})$, 
one obtains

$4 \pi \nabla \nabla: \vec{P}=-\vec{B} \nabla^{2} \vec{B}-\left(\frac{4 \pi}{c}\right)^{2} j^{2}=\mu_{n}^{2} B^{2}-\left(\frac{4 \pi}{c}\right)^{2} j^{2}$,

where $\nabla \cdot \vec{j} \times \vec{B}=c \nabla \nabla: \vec{P}$.

\section{Local variation properties of the entropy}

We shall now see how the relations (11.4) and (11.13) are related to the local variation properties of the magnetic entropy (11.1). We consider variations of (11.1) localized in a certain region $\Delta \Omega$ covering a number of small suitably chosen volume elements $\Delta \omega_{n}$. The variations are rapidly varying inside $\Delta \Omega$ and vanish on the surface of $\Delta \Omega$. Under these conditions, we expect that the entropy (11.1) is stationary with respect to a family of localized variations $\overrightarrow{\delta j}$ while $\mu_{n}^{2}$ is kept fixed, that is to say in our terminology, with respect to "isothermal" variations ( $\tau_{n}$ is constant) and that the maximum or minimum is related to the sign of $\mu_{n}^{2}$.

Indeed, let us take for $\vec{j}_{n}$ and $\vec{A}_{n}$ the averages of $\vec{j}$ and $\vec{A}$ in $\Delta \omega_{n}$, e.g. $\vec{j}_{n}=\left(1 / \Delta \omega_{n}\right) \int_{\Delta \omega_{n}} \vec{j} d \omega_{n}$. When the $\Delta \omega_{n}$ are so small and the variations $\delta j$ and $\delta A$ ( $j_{n}$ and $A_{n}$ are kept fixed) are so slow that they can be considered as practically uniform inside each $\Delta \omega_{n}$ separately, the first variation of each $S_{p n}$ (11.1) can be seen to vanish. The same holds in the opposite case when the variations are varying so rapidly in each $\Delta \omega_{n}$ with respect to the equilibrium, so that they average to zero in $\Delta \omega_{n}$. Indeed, under this condition the first variation of $S_{p}$ can be written in the forms

$$
\begin{aligned}
& \delta S_{p} \propto \sum_{n}\left(-\int_{\Delta \omega_{n}} 2 \vec{j} \cdot \delta \overrightarrow{j j} d \omega_{n}+\frac{\mu_{n}^{2} c}{4 \pi} \int_{\Delta \omega_{n}}(\vec{j} \cdot \delta \vec{A}+\vec{A} \cdot \overrightarrow{j j}) d \omega_{n}\right)= \\
& =-\int_{\Delta \Omega} 2 \vec{j} \cdot \overrightarrow{\delta j} d \Omega+\frac{c}{4 \pi} \int_{\Delta \Omega} \mu^{2}(\vec{x})(\vec{j} \cdot \delta \vec{A}+\vec{A} \cdot \overrightarrow{j j}) d \Omega \approx \\
& \approx-\int_{\Delta \Omega} 2 \vec{j} \cdot \overrightarrow{j j} d \Omega+2\left(\frac{\mu c}{4 \pi}\right)^{2} \int_{\Delta \Omega} \vec{B} \cdot \delta \vec{B} d \Omega-\left(\frac{\mu c}{4 \pi}\right)^{2} \int_{\partial \Omega} \vec{A} \times \delta \vec{B} \cdot d \vec{S}-\left(\frac{\mu C}{4 \pi}\right)^{2} \int_{\partial \Omega} \delta \vec{A} \times \vec{B} \cdot d \vec{S},
\end{aligned}
$$

where $\mu^{2}(\vec{x}) \approx \mu_{n}^{2}$ is supposed to change so slowly in $\Delta \Omega$ that its derivative can be neglected ( $\mu_{n}^{2}$ involves the second derivatives of the equilibrium magnetic field (see (11.4)) whose variation in space is assumed to be sufficiently slow). It follows that the volume integrals vanish in view of the rapid oscillations of the perturbations, while in comparison, the equilibrium quantities can be assumed as practically uniform in $\Delta \Omega$, and that the surface integrals also vanish in view of the localization of the perturbations internally localized in $\Delta \Omega$.

We take it for granted that, with the appropriate choice of the size of $\Delta \omega_{n}$, the perturbations to be considered in the following are consistent with $\delta S_{p}=0$.

As for the second variation of $S_{p}$ we have with the approximation above for $\mu^{2}(\vec{x})$

$$
\delta^{2} S_{p} \propto-\int_{\Delta \Omega} \delta j^{2} d \Omega+\frac{\mu^{2} c}{4 \pi} \int_{\Delta \Omega} \overrightarrow{\delta j} \cdot \delta \vec{A} d \Omega=-\int_{\Delta \Omega} \delta j^{2} d \Omega+\left(\frac{\mu c}{4 \pi}\right)^{2} \int_{\Delta \Omega} \delta B^{2} d \Omega .
$$


When $\mu^{2}>0$ perturbations may exist which increase the entropy and the isolated system is not thermodynamically stable. For instance, putting in (12.3) $\delta \vec{j}=(c / 4 \pi) \nabla \times \delta \vec{B}$ with $\delta \vec{j}=0$ on $\partial \Delta \Omega$ one finds that $\delta^{2} S_{p}>0$ if perturbations $\delta \vec{B}_{m}$ exist which satisfy the equation $\nabla^{2} \delta \vec{B}_{m}+k_{m}^{2} \delta \vec{B}_{m}=0$,

with $k_{m}^{2}<\mu^{2}$ and with $\nabla \cdot \delta \vec{B}_{m}=0$. In contrast, when $\mu^{2}<0$, one has $\delta^{2} S_{p}<0$.

We conclude that the magnetic entropy of a local isothermal equilibrium, with a given positive temperature, i.e. with fixed $\tau_{n} \propto-\mu_{n}^{-2} \approx-\mu^{-2}(\vec{x})>0$, is always at a maximum.

\section{The Magnetic Well}

We shall show that $B^{2}$ has a minimum whenever $\mu^{2}<0$. Indeed, in Cartesian coordinates one can write $B^{2}=\sum_{j} B_{j}^{2}$ and after deriving twice one obtains $\nabla^{2} B^{2}=2 \sum_{j, k}\left(\frac{\partial B_{j}}{\partial x_{k}}\right)^{2}+2 \vec{B} \nabla^{2} \vec{B}$.

From this and from (11.4) it follows that $\nabla^{2} B^{2}>0$, that is to say, $B^{2}$ is subarmonic, which implies that it takes its maximum on the boundary. If $B^{2}$ is constant on the boundary, it has a minimum in the interior and one has a magnetic well.

While the condition $\mu^{2}<0$ implies a magnetic well, it implies also a condition on the pressure tensor through (11.13), namely $\nabla \nabla: \vec{P}<0$. We investigate the consequences of this condition presently.

We start from the form of the pressure tensor originally introduced by Chew, Goldberger and Low [11]

$$
\vec{P}=\vec{b} \vec{b} P_{\|}+\left(\vec{I}-\vec{b} \vec{b} P_{\perp}\right),
$$

where $\vec{b}=\vec{B} / B$. It can be easily shown (see e.g. [12]) that the divergence of $\vec{P}$ can be written in the form

$\nabla \cdot \vec{P}=\nabla P_{\perp}+\vec{B} \nabla_{\|}\left(\frac{P_{\|}-P_{\perp}}{B}\right)+\vec{\kappa}\left(P_{\|}-P_{\perp}\right)$,

where $\nabla_{\|}=\vec{b} \cdot \nabla$ and $\vec{\kappa}$ denotes the curvature of the magnetic field

$\vec{\kappa}=\vec{b} \nabla \vec{b}=-\vec{b} \times \nabla \times \vec{b}=\frac{4 \pi}{c} \frac{\vec{j} \times \vec{B}}{B^{2}}+\frac{\nabla_{\perp} B}{B}=\frac{4 \pi}{B^{2}} \nabla \cdot \vec{P}+\frac{\nabla_{\perp} B}{B}$.

Here the equilibrium equation (11.11) has been used.

A useful relation is obtained by projecting (13.3) along $\vec{B}$ :

$\nabla_{\|} P_{\|}-\left(P_{\|}-P_{\perp}\right) \frac{\nabla_{\|} B}{B}=0$.

Putting $\sigma=1-4 \pi\left(P_{\|}-P_{\perp}\right) / B^{2}$ and taking into account (13.4) and (13.5), (13.3) becomes

$\nabla \cdot \vec{P}=\frac{1}{\sigma}\left(\nabla P_{\perp}-\vec{b} \nabla_{\|} P_{\perp}+\frac{\nabla_{\perp} B}{B}\left(P_{\|}-P_{\perp}\right)\right)$.

In order to proceed with the calculation of $\nabla \nabla: \vec{P}$ in the case of the magnetic well, we introduce the assumption that $P_{\|}$and $P_{\perp}$ depend on space only through $B$, that is to say, $P_{\|}=P_{\|}(B), P_{\perp}=P_{\perp}(B)$ 
(this assumption implies that the distribution function of the equilibrium depends only on the kinetic energy, $\varepsilon=(m / 2)\left(v_{\perp}^{2}+v_{\|}^{2}\right)$ and on the magnetic moment $\mu=\left(m v_{\perp}^{2} / 2 B\right)$ [13]). Then eq.(13.5) becomes $d P_{\|} / d B=\left(P_{\|}-P_{\perp}\right) / B$,

and (13.6) takes the simple form

$\nabla \cdot \vec{P}=\frac{1}{\sigma} \frac{d P}{d B} \nabla_{\perp} B$

where $P=P_{\|}+P_{\perp}$. It follows

$\nabla \nabla: \vec{P}=-\frac{1}{\sigma^{2}} \frac{d P}{d B} \nabla_{\perp} B \cdot \nabla\left(\frac{4 \pi}{B^{2}}\left(P_{\perp}-P_{\|}\right)\right)+\frac{1}{\sigma}\left(\frac{d^{2} P}{d B^{2}}\left(\nabla_{\perp} B\right)^{2}+\frac{d P}{d B} \nabla \cdot \nabla_{\perp} B\right)=$

$\frac{1}{\sigma^{2}}\left(\frac{8 \pi}{B^{3}}\left(\nabla_{\perp} B\right)^{2}\left(P_{\perp}-P_{\|}\right) \frac{d P}{d B}-\frac{4 \pi}{B^{2}} \frac{d P_{\perp}}{d B} \frac{d P}{d B}\left(\nabla_{\perp} B\right)^{2}+4 \pi\left(\frac{P_{\|}-P_{\perp}}{B^{2}}\right) \frac{d P}{d B}\left(\nabla_{\perp} B\right)^{2}\right)+$

$+\frac{1}{\sigma}\left(\frac{d^{2} P}{d B^{2}}\left(\nabla_{\perp} B\right)^{2}+\frac{d P}{d B} \nabla \cdot \nabla_{\perp} B\right)$

We observe that

$\nabla \cdot \nabla_{\perp} B=\nabla \cdot\left(\nabla B-\vec{b}\left(\nabla_{\|} B\right)\right)=\nabla^{2} B-\nabla \cdot\left(\vec{b}\left(\nabla_{\|} B\right)\right)=\nabla^{2} B-\vec{b} \cdot \nabla\left(\nabla_{\|} B\right)-\nabla_{\|} B \nabla \cdot \vec{b}=$

$=\nabla^{2} B-\nabla^{2}{ }_{\|} B+\frac{\left(\nabla_{\|} B\right)^{2}}{B}$.

Using this relation and (13.7) one obtains

$\nabla \nabla: \vec{P}=-\frac{1}{\sigma^{2}}\left(\frac{4 \pi}{B^{2}}\left(\nabla_{\perp} B\right)^{2} \frac{d P}{d B} \frac{d P_{\|}}{d B}+\frac{\left(\nabla_{\perp} B\right)^{2}}{B^{2}}\right)+\frac{1}{\sigma}\left(\frac{d^{2} P}{d B^{2}}\left(\nabla_{\perp} B\right)^{2}+\frac{d P}{d B} \nabla \cdot \nabla_{\perp} B\right)=$

$\left.-4 \pi\left(\frac{1}{\sigma} \mid \nabla_{\perp} B \frac{d P}{d B}\right)\right)^{2}+\frac{1}{\sigma}\left(\frac{d^{2} P}{d B^{2}}\left(\nabla_{\perp} B\right)^{2}+\frac{d P}{d B}\right)\left(\nabla_{\perp}^{2} B+\frac{\left(\nabla_{\|} B\right)^{2}}{B}\right)$.

In order that the entropy is not at a minimum this form must be positive definite. Since $B$ has a minimum, as we know from above, one must have $\nabla_{\perp}^{2} B>0$ in a region around the minimum. Then the form (13.11) is positive definite if the following conditions are satisfied in this region: 1) $\sigma>0 ; 2$ ) $d P / d B \leq 0$; 3) $d^{2} P / d B^{2}<0$. The first condition implies the absence of the fire-hose instability, which exists only in the opposite exceptional case [14]

$P_{\|}-P_{\perp}>\frac{B^{2}}{4 \pi}$.

The conditions 2) and 3) imply that, if $B$ is constant at the boundary, $P(B)$ decreases everywhere in the region around the minimum $B$ apart from a point where it is at a maximum.

The conditions above were first derived, in the case of vanishing parallel current, by Santini [15] directly from the condition that the quadratic form $\mu^{2} \propto d \vec{j} \cdot d \vec{A}=(1 / 2)(\xi \cdot \nabla \vec{j}) \cdot(\vec{B} \times \vec{\xi})$ be negative definite (see [16]).

\section{The Interchange Instability}

The sign of the quadratic form $(\vec{\xi} \cdot \nabla \vec{j}) \cdot(\vec{B} \times \vec{\xi})$ plays a fundamental role in our considerations and it is then worthwhile to note that it can be seen from another point of view, susceptible of generalizations. 
We start from the macroscopic magnetic energy $\Phi=(1 / 2 c) \int \vec{j} \cdot \vec{A} d \Omega$ and consider the energy $\Delta \Phi_{i}$ contained in two small adjacent volume elements $\Delta \omega_{1}$ and $\Delta \omega_{2}$ (not necessarily flux tube elements) centered at two points 1 and 2 displaced along a vector $\xi_{\perp}$ in a direction perpendicular to $\vec{B}$. Choosing the volume elements small enough and each equal to $\Delta \omega$, one has

$\Delta \Phi_{i}=\frac{\Delta \omega}{2 c}(\vec{j}(1) \cdot \vec{A}(1)+\vec{j}(2) \cdot \vec{A}(2))$

Now we consider the new configuration in which the values of the current density in 1 and 2 have been interchanged while the vector potential $\vec{A}$ was kept constant at each point. After this interchange the expression (14.1) has to be replaced by

$\Delta \Phi_{f}=\frac{\Delta \omega}{2 c}(\vec{j}(2) \cdot \vec{A}(1)+\vec{j}(1) \cdot \vec{A}(2))$

Clearly, in a low $\beta$ approximation one can indeed change the current distribution $\vec{j}(x)$ without changing the magnetic field and its vector potential $\vec{A}$. However the same result (14.2) could also be obtained with a more flexible procedure in which the values of the vector potential in 1 and 2 are interchanged, whereas the quantity $\Delta \omega \vec{j}(x)$ remains fixed at each point. In this way $\Delta \omega$ and $\vec{j}(\vec{x})$ can change separately in order that $\vec{j}$ is consistent with the variation of the magnetic field configuration. The energy variation due to the interchange considered above is then

$\delta^{2} \Phi=\Delta \Phi_{f}-\Delta \Phi_{i}=-\frac{\Delta \omega}{2 c}(\vec{j}(2)-\vec{j}(1)) \cdot(\vec{A}(2)-\vec{A}(1))=-\frac{\Delta \omega}{2 c} d \vec{j} \cdot d \vec{A}$.

Restricting ourselves to first order in $\vec{\xi}_{\perp}$ we can write

$\vec{A}(2)-\vec{A}(1)=\frac{1}{2} \vec{B}(1) \times \vec{\xi}_{\perp}, \quad \vec{j}(2)-\vec{j}(1)=\vec{\xi}_{\perp} \cdot \nabla \vec{j}(1)$,

and $\delta^{2} \Phi$ becomes

$\delta^{2} \Phi=-\frac{\Delta \omega}{4 c}\left(\vec{\xi}_{\perp} \cdot \nabla \vec{j}(1)\right) \cdot(\vec{B}(1) \times \vec{\xi})$

It follows that when the interchange energy is minimum ( $\left.\delta^{2} \Phi>0\right) \mu_{n}^{2}$ (defined by (11.2) with $\Phi_{\text {int }}=0$ ) is negative, the expression (12.3) for $\delta^{2} S_{p}$ is also negative and the entropy is at a maximum.

In addition to its relation with the variation properties of the entropy, the interchange variation (13.5) has an interest in itself. Indeed one can discuss the stability of the magnetic equilibrium in a variety of physical situations depending on the special form of the current density to be introduced in (14.5).

For instance, in the case of the low- $\beta$ interchange instabilities, the process is driven by the current, which arises from the opposite drift of ions and electrons. As a simple example let us consider the case of the inhomogeneous plasma in a constant magnetic field $\vec{B}=(0,0, B)$, with ions and electrons subject to a gravitational force $m_{s} g \vec{e}_{x}$ (the subscript $s$ refers to ions or electrons). In this configuration the particles drift in the direction $\vec{e}_{y}$ with the velocity

$v_{D y}=-\frac{m_{s} c g}{q_{s} B}$,

which gives rise to a current density

$j_{s, y}=-q_{s} n_{s} v_{D y}=-\frac{m_{s} n_{s} c g}{B}$.

Noting that $d A_{y}=B d x$ one has

$\delta^{2} \Phi \propto-d j_{s, y} d A_{y}=\frac{m_{s} c g}{B} \frac{d n_{s}}{d x}(d x)^{2}$, 
and this quantity is positive when $n(x)$ increases in the direction of gravity, which implies, on one hand, that $\Phi$ is at minimum and the system is stable, and, on the other, that the entropy is maximum with respect to all perturbations (see (12.3)).

We can extend this reasoning to the drift current density arising from the non-homogeneity of the magnetic field (see [11], p145):

$\vec{j}_{d}=c \frac{\vec{B} \times \nabla B}{B^{3}}\left(P_{\|}+P_{\perp}\right)$.

In the calculation of $\xi \cdot \nabla \overrightarrow{j_{d}}$ we neglect all terms involving $P / B^{2}$ with respect to the term involving $\vec{\xi} \cdot \nabla P$ where $P=P_{\|}+P_{\perp}$. In particular we assume $|\nabla P / P|<<|\nabla B / B|$. Then one has the equality

$d j_{d} \cdot d \vec{A}=c \vec{\xi} \cdot \nabla\left(\frac{P \vec{B} \times \nabla B}{B^{3}}\right) \cdot\left(\frac{\vec{B} \times \vec{\xi}}{2}\right) \approx \frac{\vec{\xi} \cdot \nabla P(\vec{B} \times \nabla B) \cdot(\vec{B} \times \vec{\xi})}{2 B^{3}}=\frac{\left(\vec{\xi}_{\perp} \cdot \nabla P\right)\left(\vec{\xi}_{\perp} \cdot \nabla B\right)}{2 B}$.

For $\beta \equiv 4 \pi P / B^{2}<<1, \vec{\kappa} \cdot \vec{\xi}_{\perp}$ can be approximated by $\nabla B \cdot \vec{\xi}_{\perp} / B$. In the case of a scalar pressure $p=P_{\|}=P_{\perp}$, so

$\delta^{2} \Phi=-\frac{\Delta \omega}{2 c} d \vec{j} \cdot d \vec{A}=-\frac{\Delta \omega}{2 c}\left(\vec{\xi}_{\perp} \cdot \nabla p\right)\left(\vec{\xi}_{\perp} \cdot \vec{\kappa}\right)$

This term is the same as that describing the low- $\beta$ interchange instability in the magnetohydrodynamic energy principle (see e.g. [17]).

\section{Thermodynamic interpretation of the tearing modes}

In a situation in which the magnetic system is subject to dissipation and part of the magnetic energy decays, the energy transfer can be taken into account in a phenomenological way by the variation of the interaction energy $\Phi_{\text {int }}$. That is to say, the macroscopic system is not isolated and energy is transferred from the system to the background (or to the external world). This is the case of the tearing modes, which we are going to discuss presently.

As is known from the conventional treatment of the tearing modes, one distinguishes two physical regions in the plasma: a resistive layer localized on a rational magnetic surface inside which dissipation is taking place and a non-dissipative outer region.

We consider the plasma in cylindrical geometry carrying in the unperturbed equilibrium an axial current density $j_{\phi}(r)$ that creates a vector potential $A_{\phi}(r)$. The plasma is situated in a uniform axial magnetic field $B_{\phi}$ so large to be practically insensitive to small perturbations, while the other components can be slightly helically perturbed. As is well known, in view of the helical symmetry, the current density of the perturbed system is a function of space only through the helical flux $\chi=-m A_{\phi}(r, \theta, \phi)-n r A_{\theta} / R$ where $A_{\theta}=r B_{\phi} / 2$ and $m, n$ are the poloidal and toroidal wave numbers associated with the rational magnetic surface under consideration. Let us divide the plasma volume into small sectors of cylindrical shells $\Delta \omega_{n}$ and let $\vec{x}_{n} \equiv\left(r_{n}, \theta_{n}, \phi_{n}\right)$ be the co-ordinates of some point inside $\Delta \omega_{n}$. The co-ordinates of a point varying inside the sector of the shell with very small radial thickness $\Delta r$ and small radial and angular ranges $\Delta \theta$ and $\Delta \varphi$ are $\vec{x} \equiv\left(r_{n}, \theta, \phi\right)$ where $\vec{x} \in \Delta \omega_{n}$. Thus we can write inside the small shell (henceforth dropping the subscript $\phi$ )

$j-j_{n}=\frac{d j}{d A_{n}}\left(A-A_{n}\right)=-\frac{d j}{d \chi_{n}} m\left(A-A_{n}\right)$,

where the subscript $n$ denotes the value of the quantities at the point $\vec{x}_{n}$. 
It follows from (11.2) (with $\Phi_{\text {int }}=0$ at the zero order initial value) that

$\mu_{n}^{2}=-\frac{4 \pi m}{c} \frac{d j}{d \chi_{n}}$.

Let us calculate the first variations of $S_{p}$ and of $S_{b}=\sum_{n} \Phi_{n \text {,int }} / \tau_{n}$ with respect to generic primary variations $\delta j$ and $\delta A$ (where $\left.\delta j=-(c / 4 \pi) \nabla^{2} \delta A\right)$, keeping $\tau_{n}$ fixed at the zero order initial value:

$$
\begin{aligned}
& \delta S_{p}=-\frac{3}{2 \Delta V \overline{\tilde{j}^{2}}} \sum_{n} \int_{\Delta \omega_{n}}\left(\delta j\left(2\left(j-j_{n}\right)-\frac{c \mu_{n}^{2}}{4 \pi}\left(A-A_{n}\right)\right)-\frac{c \mu_{n}^{2}}{4 \pi} \delta A\left(j-j_{n}\right)\right) d \omega_{n}= \\
& =-\frac{3}{2 \Delta V \overline{\tilde{j}^{2}}} \sum_{n} \int_{\Delta \omega_{n}}\left(j-j_{n}\right)\left(\delta j-\frac{c \mu_{n}^{2}}{4 \pi} \delta A\right) d \omega_{n},
\end{aligned}
$$

$$
\begin{aligned}
& \delta S_{b}=\sum_{n} \frac{\delta \Phi_{\text {int }}}{\tau_{n}}=-\frac{1}{2 c} \sum_{n} \frac{1}{\tau_{n}} \int_{\Delta \omega_{n}}\left(A-A_{n}\right)\left(\delta j+m \frac{d j}{d \chi_{n}} \delta A\right) d \omega_{n}= \\
& =\frac{3}{2 \Delta V \overline{\tilde{j}^{2}}} \sum_{n} \int_{\Delta \omega_{n}}\left(j-j_{n}\right)\left(\delta j-\frac{c \mu_{n}^{2}}{4 \pi} \delta A\right) d \omega_{n} .
\end{aligned}
$$

It follows that $\delta S=\delta S_{p}+\delta S_{b}=0$. For the second variations one obtains

$$
\delta^{2} S_{p}=-\frac{3}{2 \Delta V \overline{\widetilde{j}^{2}}} \sum_{n} \int_{\Delta \omega_{n}} \delta j\left(\delta j-\frac{c \mu_{n}^{2}}{4 \pi} \delta A\right) d \omega_{n},
$$

$$
\begin{aligned}
& \delta^{2} S_{b}=\frac{1}{2 c} \sum_{n} \frac{\delta^{2} \Phi_{n, \text { int }}}{\tau_{n}}=-\frac{1}{2 c} \sum_{n} \frac{1}{\tau_{n}} \int_{\Delta \omega_{n}} \delta A\left(\delta j-\frac{c \mu_{n}^{2}}{4 \pi} \delta A\right) d \omega_{n}= \\
& =\frac{3 c}{8 \pi \widetilde{j}^{2} \Delta V} \sum_{n} \mu_{n}^{2} \int_{\Delta \omega_{n}} \delta A\left(\delta j-\frac{c \mu_{n}^{2}}{4 \pi} \delta A\right) d \omega_{n} .
\end{aligned}
$$

so that

$$
\delta^{2} S=\delta^{2} S_{p}+\delta^{2} S_{b}=-\frac{3}{2 \Delta V \widetilde{\tilde{j}^{2}}} \sum_{n} \int_{\Delta \omega_{n}}\left(\delta j-\frac{c \mu_{n}^{2}}{4 \pi} \delta A\right)^{2} d \omega_{n} \leq 0 .
$$

As already noted (section 9) the total system formed by the collective equilibrium plus background, is thermodynamically stable because the entropy $S$ is extreme and maximum, the adiabatic variations $\delta j=\left(c \mu_{n}^{2} / 4 \pi\right) \delta A$ apart. But instability can arise for the collective magnetic subsystem when the variation of the interaction energy with the background is positive because this means that magnetic energy is transferred from the collective (macroscopic) magnetic system to the background (or to the external world). In this case the collective system is not isolated but is allowed to interact and decay by exchanging energy externally to the system. This is indeed the case of the tearing modes where the dissipative layer acts as a sink of magnetic energy.

Let $\Delta \omega_{s}$ be the site of dissipation, namely a cylindrical shell centered on the rational surface $q(s)=m / n$ with infinitesimal radial thickness $2 \varepsilon$. Outside $\Delta \omega_{s}$ the variations are assumed to be adiabatic and reversible so that $\delta S_{p}=\delta S_{b}=\delta^{2} S=0$ and, according to (15.3) and (15.4), $\delta A$ must satisfy the equation

$$
\nabla^{2} \delta A-\frac{4 \pi m}{c} \frac{d j}{d \chi_{n}} \delta A=0
$$


for any $n$ in the outer region. This is just the equation of the tearing modes in the outer region.

Inside the dissipation layer the second variation (15.5) of the entropy $S_{p}$ is singular as a consequence of the singularity of $\mu_{n} \propto-\tau_{n}^{-1}$ at $r=s$. For getting a finite result one should take into account the non-linear effects in the evolution of the tearing mode that resolve the singularity and lead to the formation of the magnetic island. However, the variations of the magnetic energy $\Phi$ and of the interaction energy $\Phi_{\mathrm{int}}$, are not affected by the singularity of $\mu_{n}$ at $q=m / n$, and $\tau_{n} \delta^{2} S_{p}$ remains finite. So we can discuss the thermodynamic interpretation of these quantities while remaining in the linear approximation.

We then proceed to calculate the variation of the magnetic energy $\Phi_{s}=(1 / 2 c) \int_{\Delta \omega_{s}}\left(j-j_{s}\right)\left(A-A_{s}\right) d \omega_{s}$ localized in $\Delta \omega_{s}$. The first variation is easily seen to vanish because $j \rightarrow j_{s}, A \rightarrow A_{s}$ for $\varepsilon \rightarrow 0$. The second variation is given as follows:

$\delta^{2} \Phi_{s}=\frac{R s}{c} \iint d \theta d \varphi \int_{s-\varepsilon}^{s+\varepsilon} \delta j \delta A r d r=-\frac{R}{4 \pi} \iint d \theta d \varphi \int_{s-\varepsilon}^{s+\varepsilon} \delta A\left(\frac{d}{d r}\left(r \frac{d \delta A}{d r}\right) d r\right)$.

The derivative $d \delta A / d r$ is discontinuous in $r=s$. In view of the integration in (15.9) we represent formally the second derivative of $\delta A$ in the neighborhood of $r=s$ with the help of the Dirac $\delta$ function

$\frac{d^{2} \delta A}{d r^{2}}=\frac{d^{2} \delta A}{d r^{2}}+\left(\left(\frac{d \delta A}{d r}\right)_{s+\varepsilon}-\left(\left(\frac{d \delta A}{d r}\right)_{s-\varepsilon}\right)\right) \delta(r-s)$.

In the limit $\varepsilon \rightarrow 0$ only the $\delta$-function contributes to the integral (15.9) and one obtains

$\delta^{2} \Phi_{s}=-\frac{R s}{4 \pi} \iint \Delta^{\prime}(\delta A(s))^{2} d \theta d \varphi$

where

$\Delta^{\prime}=\left|\frac{d \delta A}{d r}\right|_{s-\varepsilon}^{s+\varepsilon} / \delta A(s)$

When $\delta^{2} \Phi_{s}<0$ the system is unstable and $\Delta^{\prime}>0$.

The variation $\delta^{2} \Phi_{s}$ is related to $\delta^{2} \Phi_{s, \text { int }}$. Indeed recalling (15.6) one has in the limit $\varepsilon \rightarrow 0$ $\delta^{2} \Phi_{s, \text { int }}=-\delta^{2} \Phi_{s}$

noting that the part of the integral involving $\delta A^{2}$ vanishes in this limit.

According to our model, $\Phi_{\text {int }}$ describes the energy interaction of the collective magnetic system with the external world. The relation (8.3) shows that a positive change of $\Phi_{\text {int }}$ describes the Poynting flux of electromagnetic energy lost by the collective system. So, consistently with (8.3), we put $\delta^{2} \Phi_{s, \text { int }}=-\delta Q$,

where $\delta Q>0$ denotes generically the energy supplied to the magnetic system.

Then one sees that in the unstable case $\Delta^{\prime}>0, \delta^{2} \Phi_{s}<0$, energy is transferred from the magnetic system to the background, as described by (15.12) with $\delta^{2} \Phi_{s, \text { int }}>0$, and lost, as described by (15.13). By considering the system as isolated the decay energy is absorbed internally to the system in a irreversible process.

In the outer region where $\delta Q=0$, the energy conservation is expressed by $\delta^{2} \Phi_{\text {out }}+\delta L=0$ where $\delta^{2} \Phi_{\text {out }}$ is the outer variation of the magnetic energy and $\delta L$ is the work of internal inductive electric fields and plasma currents when the system can be considered as isolated, or otherwise, is due to the 
mutual interaction with the external circuits that react when the magnetic plasma configuration is varying.

\section{CHAPTER 4: Minimum Principles}

Summary: The pressure profiles in tokamaks are restricted by the requirement that the thermal energy of the plasma be minimum for fixed magnetic entropy and fixed total current.

The poloidal magnetic energy is minimized, within the family of states that satisfy the SME equation in the presence of auxiliary heating, when the parameter $\mu a$ tends to zero ( $a$ is the minor radius), even though, for reason of mathematical consistency, it cannot be put identically equal to zero. The validity of these principles rests on the experimental verification of their predictions on the SME profiles (chapter 6).

The assumption of stationary magnetic entropy is a crucial ingredient in the formulation of both principles.

\section{Minimum plasma thermal energy and pressure profile in tokamaks}

The pressure profile is expressed in terms of the poloidal flux $\psi$, which is determined in the cylindrical limit by the D’Alembert equation

$\nabla^{2} \psi=\frac{4 \pi R}{c} j_{\text {tot }}$,

where $j_{\text {tot }}$ is given by (7.8), $\psi=-R A_{\phi}$ ( $A_{\phi}$ is the axial component of the vector potential and

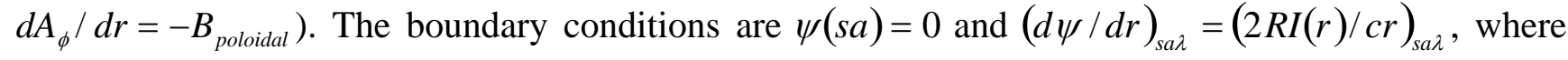
$I(r)$ is the total current flowing inside $r$, sa and $s a \lambda$ are the outer and inner borders of the relaxed region respectively.

One has a simple relation between $\psi$ and the solution $j$ of eq.(7.4). Taking into account (7.8) and (16.1), eq.(7.4) becomes (with $E=E_{\text {ext }}$ )

$$
\nabla^{2}\left(j+\frac{c \mu^{2}}{4 \pi R} \psi\right)=-\frac{\mu^{2}}{E}\left(p_{E}-E j_{\text {nind }}\right) .
$$

After a double integration one obtains

$$
j=-\frac{c \mu^{2} \psi}{4 \pi R}-(\mu \mathrm{s} a)^{2} \int_{\lambda}^{x} \frac{d x}{x} \int_{0}^{x} \pi_{E}(x) x d x+D_{0} \lg x+D_{1} .
$$

Here $x=r / s a, D_{0}$ and $D_{1}$ are integration constants to be fixed by the two boundary conditions on the current density and

$$
\pi_{E}(x)=\frac{p_{E}(x)-E j_{\text {nind }}(x)}{E} .
$$

The pressure profile is determined assuming that the plasma thermal energy in the confinement zone

$$
W=\frac{3}{2} \int p(\psi) d \Omega
$$


is stationary (it turns out to be minimum in the practical cases) with respect to variations $\delta \psi$ in the confinement region such that the total induced current $I_{\text {ind }}=\int 2 \pi j r d r$ and the magnetic entropy (defined by (5.4) with the induced current density (16.3)) are fixed $\delta\left(W+\eta_{1} S_{p}+\eta_{2} I_{\text {ind }}\right)=0$,

where

$\delta W=(3 / 2) \int_{\text {sad }}^{s a} p(\psi) \delta \psi 4 \pi^{2} R r d r$

$\delta S_{p}=-8 \pi^{2} R \int_{s a \lambda}^{s a} j \delta j r d r-c \mu^{2} \pi \int_{s a \lambda}^{s a} \delta(j \psi) r d r$

$\delta I=2 \pi \int_{s a \lambda}^{s a} \delta j r d r$

and $\delta j=-\left(c \mu^{2} / 4 \pi R\right) \delta \psi$. Note that, as observed in section 9, the perturbed and unperturbed current densities $j+\delta j$ and $j$ satisfy the same SME equation (7.4). The factors $\eta_{1}$ and $\eta_{2}$ are Lagrange multipliers and the inessential positive factor of $S_{p}$ has been absorbed in $\eta_{1}$.

In view of the arbitrariness of $\delta \psi$ the variation condition (16.6) leads to the expression

$\frac{d p}{d \psi}=\eta_{1}\left(\frac{c \mu^{4}(s a)^{2}}{6 \pi R} \int_{1}^{x} \frac{d x}{x} \int_{0}^{x} \pi_{E}(x) x d x-\frac{c \mu^{2} D_{0}}{6 \pi R} \lg (x)-\frac{c \mu^{2} D_{1}}{6 \pi R}\right)+\eta_{2} \frac{c \mu^{2}}{12 \pi^{2} R^{2}}$.

The integration of this equation gives $p(x)$ once $\psi(x)$ is known $(\psi(x)$ is calculated from (16.1) after solving (7.4) for $j$ and taking into account (7.8). There are three constants to be determined, namely $\eta_{1}, \eta_{2}$ and the integration constant of (16.10) (the constants $D_{0}$ and $D_{1}$ are fixed by the boundary conditions on the current density). The values of $p$ at the two boundaries of the integration zone (to be taken from the experiment) leave us with one constant free. This constant can be expressed in terms of the plasma thermal energy in the integration zone. Indeed integration of (16.10) gives

$$
p(\Psi)=\eta_{1} \int_{0}^{\Psi} d \Psi B\left(\frac{(\mu s a)^{2} c}{3 \pi R} \int_{1}^{x(\Psi)} \frac{d x}{x} \int_{0}^{x} \pi_{E}(x) x d x-\frac{c D_{0}}{3 \pi} \ln (x(\Psi))\right)+p_{s}^{\prime} \Psi+p_{s},
$$

where $\Psi=\left(\mu^{2} / 2 B\right) \psi, p_{s}$ is the value of $p$ at $x=1, \Psi=0$ and

$p_{s}^{\prime}\left(\eta_{1}, \eta_{2}\right)=\left(\frac{d p}{d \Psi}\right)_{x=1}=B\left(\frac{\eta_{2} c}{6 \pi^{2} R^{2}}-\frac{\eta_{1} c D_{1}}{3 \pi R}\right)$.

Let us put

$X(\Psi)=-\int_{0}^{\Psi} d \Psi B\left(\frac{(\mu s a)^{2} c}{3 \pi R} \int_{1}^{x(\Psi)} \frac{d x}{x} \int_{0}^{x} \pi_{E}(x) x d x-\frac{c D_{0}}{3 \pi} \ln (x(\Psi))\right)$.

Then the pressure takes on the form $p(\Psi)-p_{s}=-\eta_{1} X(\Psi)+p_{s}^{\prime} \Psi$.

Denoting with the caps the values at $x=\lambda$ one has from (16.14) that $p_{s}^{\prime}=\left(\eta_{1} \hat{X}+\hat{p}-p_{s}\right) / \hat{\Psi}$. Then (16.14) can be rewritten as

$$
p(\Psi)-p_{s}=\eta_{1} Y(\Psi)+\frac{\Psi}{\hat{\Psi}}\left(\hat{p}-p_{s}\right)
$$

with $Y(\Psi)=\hat{X} \Psi / \hat{\Psi}-X(\Psi)$.

In order to determine $\eta_{1}$ we integrate (16.15) over the volume of the relaxed region, obtaining 
$\eta_{1}=\frac{1}{\bar{Y}}\left(\bar{p}-p_{s}-\frac{\bar{\Psi}}{\hat{\Psi}}\left(\hat{p}-p_{s}\right)\right)$

Let us introduce the parameter $K$ defined by the equality

$K=1+\frac{\hat{X}}{\bar{Y}}\left(\overline{p_{N}}-\frac{\bar{\Psi}}{\hat{\Psi}}\right)$,

with $\overline{p_{N}}=\bar{p} / \hat{p}$. The parameter $\eta_{1}$ becomes

$\eta_{1}=\frac{\hat{p}}{\bar{Y}}\left((K-1) \frac{\bar{Y}}{\hat{X}}+p_{s N}\left(\frac{\bar{\Psi}}{\hat{\Psi}}-1\right)\right) \approx \hat{p} \frac{K-1}{\hat{X}}$,

where $p_{s N}=p_{s} / \hat{p}<<1$ and

$\hat{X}=\frac{c B D_{0}}{3 \pi} \int_{0}^{\hat{\Psi}} d \Psi \ln x d x$

To see the sign of the second variation (16.7)

$\delta^{2} W=3 \int_{s a \lambda}^{s a} p^{\prime \prime}(\psi) \delta \psi^{2} \pi^{2} R r d r$

we calculate from (16.14) the second derivative of $p(\Psi)$

$p^{\prime \prime}(\Psi)=-\eta_{1} X^{\prime \prime}(\Psi)=-\eta_{1}\left(-\frac{B c(\mu s a)^{2}}{3 \pi R} \frac{d x}{d \Psi} \frac{1}{x} \int_{0}^{x} \pi_{E}(x) x d x+\frac{B c D_{0}}{3 \pi} \frac{1}{x} \frac{d x}{d \Psi}\right)$.

We anticipate that the comparison with the observations shows invariably that the value of the parameter $(\mu s a)^{2}$ has an upper limit much lower than one. The justification of this will be given in the next section. Thus the leading term of (16.21) becomes

$p^{\prime \prime}(\Psi)=-\eta_{1} X^{\prime \prime}(\Psi)=-\eta_{1} \frac{c B D_{0}}{3 \pi} \frac{1}{x} \frac{d x}{d \Psi}=-\eta_{1} \frac{B^{2} c^{2} D_{0}}{3 \pi \mu^{2} R I(\Psi)}$,

where $I(\Psi)$ is the current flowing inside $\Psi$. Applying (16.18) and (16.19) one has from (16.22) that

$p^{\prime \prime}(\Psi)=\hat{p} \frac{1-K}{\hat{X}} \frac{c^{2} B^{2} D_{0}}{3 \pi \mu^{2} R I(\Psi)}=\hat{p}(1-K) \frac{c B}{\mu^{2} R I(\Psi)} \frac{1}{\int_{0}^{\Psi} d \Psi \ln x}$.

This expression can be rewritten in terms of the conventional dimensional poloidal flux $\psi=\left(2 B / \mu^{2}\right) \Psi$

$p^{\prime \prime}(\psi)=\frac{\mu^{4}}{4 B^{2}} p^{\prime \prime}(\Psi)=\hat{p} c \frac{(1-K)}{2 R I(\psi)} \frac{1}{\int_{0}^{\hat{\psi}} d \psi \ln x}$.

The integral at the denominator is positive. Indeed, (recalling that $d \psi / d x>0$ )

$\int_{0}^{\pi} d \psi \ln x=-\int_{\lambda}^{1} \frac{d \psi}{d x} \ln x d x>0$.

It follows that $\delta^{2} W>0$ for $K<1$, so that $W$ is at a minimum.

The parameter $K$ is related to the width $\overline{p_{N}}$ and to the concavity of the pressure profile. As we shall see (chapter 6 ) the observed pressure profiles are almost invariably concave in the region where the SME theory holds, in agreement with (16.24). 
We are then restricted by the variation procedure above to a family of normalized pressure profiles, which depends on the parameter $\overline{p_{N}}$ and (to a lesser extent) on the normalized value $p_{s N}$ at the outer border. Note that by combining (16.11) with (16.3) one obtains a relation between the pressure and the current density and consequently a relation between $\overline{p_{N}}$ and the average of the current in the relaxation zone.

\section{Minimum poloidal magnetic energy and profile consistency of the SME states}

Let us investigate whether one can select among the solutions of the SME equation (7.4), those associated with a minimum of the poloidal magnetic energy $w=(1 / 2 c) \int j A_{\phi} d \Omega$ (recall that $j=j_{\phi}$ is the axial current density)

We start by observing that the solution of the SME equation can be written in the form $j=j_{\text {ho }}+j_{\text {inho }}$ where $j_{\text {ho }}$ satisfies eq.(7.4) without the inhomogeneous term, and $j_{\text {inho }}$ is a particular solution of the inhomogeneous equation. The part $j_{h o}$ contains two arbitrary constants to be determined by the boundary conditions, while, as can be easily verified, the part $j_{\text {inho }}$ is formed by terms of order ( $\mu$ sa $)^{2}$ and $(\mu s a)^{2} \ln \mu s a$ lower than the part $j_{h o}$ in the limit $\mu s a \rightarrow 0$. It follows that the effect of the inhomogeneous term disappears in the integral (16.3) of the SME equation when $\mu^{2}$ is sufficiently low. In fact the comparison of the SME profiles with the observations shows invariably that they agree, in the expected region of validity, only by choosing $(\mu s a)^{2} \rightarrow 0$ (chapter 6 ).

For seeing the physical meaning of this fact let us express the poloidal magnetic energy $w$ in terms of the vector potential $A_{\phi}$ redefined according to the equality

$A_{\phi}=-\frac{\psi}{R}+\left(\frac{4 \pi}{\mu^{2} c}\right)\left(D_{0} \ln x+D_{1}\right)$,

with $\nabla^{2} A_{\phi}=-(4 \pi / c) j$. By replacing $\psi$ with $A_{\phi}$ in the expression (16.3) for $j$, one obtains

$j=\frac{\mu^{2} c}{4 \pi} A_{\phi}-(\mu s a)^{2} \int_{\lambda}^{x} \frac{d x}{x} \int_{0}^{x} \pi_{E}(x) x d x$.

Then $w$ takes on the form

$w=\frac{1}{2 c} \int_{\Omega} j A_{\phi} d \Omega=\frac{2 \pi}{\mu^{2} c^{2}}\left(\int_{\Omega} j^{2} d \Omega+\int_{\Omega} d \Omega j(\mu s a)^{2} \int_{\lambda}^{x} \frac{d x}{x} \int_{0}^{x} \pi_{E}(x) x d x\right)$.

In the presence of auxiliary heating, the term involving $\pi_{E}$ is positive, but this term disappears in the limiting process $\mu \mathrm{s} a \rightarrow 0$. Also the dependence of $j^{2}$ on $\mu$ sa disappears for $\mu$ sa sufficiently small (see chapter 6). It follows that the limiting process minimizes the poloidal magnetic energy. In contrast, in the purely ohmic case, $\pi_{E}$ describes non-diffusive losses and it is negative and the poloidal magnetic energy is maximized by the limiting process. These facts are at the root of the profile consistency of the SME states (the term "profile consistency" has been used for the first time by Coppi [18]). Indeed, one can ignore the integral term involving $\pi_{E}$ in the equation (16.3) and write approximately (recalling (17.1))

$j \approx \frac{\mu^{2} c}{4 \pi} A_{\phi}$. 
As a consequence, one can put $\varphi=0$ in eq. (8.4) when $A_{\phi}$ is defined as above, and the interaction energy (8.3) becomes identical to the Poynting term.

The relation (17.3) is associated with the extreme of the part of the magnetic entropy (5.4) that depends on $j_{\phi}$ and $A_{\phi}$. So the limiting process $\mu$ s $a \rightarrow 0$ implies that both the poloidal magnetic energy and the magnetic entropy pertaining to the axial current density are extreme.

The role played by the parameter $(\mu s a)^{2}$ in tokamaks is subtle. One cannot put $\mu$ identically equal to zero because this would be inconsistent with the relation (5.3) of $\mu^{2}$ to the Lagrange multiplier $\tau$ and would also be inconsistent with the fact that $\mu^{2}$ appears at the denominator of the power balance equation (see eq. (7.1) which is identical to the power balance (7.2) when (7.3) and $F=p_{E}$ are taken into account). While the effect of the inhomogeneous term in the solution of $j$ to the SME equation can be neglected, it must be maintained in the integral of the power balance equation (7.1), which is divided by $\mu^{2}$. Indeed, even though $\mu^{2}$ enters explicitly in the expression (7.3) for the heat flux, its effect is balanced by the implicit dependence contained in the derivative of $j_{\phi}$ and in the boundary condition for the continuity of the heat flux. These facts insure that the heat flux compensates in the relaxed region the energy input described by $p_{E}$, which is independent of $\mu$. We shall return to this point in section 20 with an explicit example.

\section{CHAPTER 5: SME states in toroidal geometry}

Summary: The SME equation is re-derived by assuming that the ohmic transformer and the source of energy of the primary (for instance, a battery of capacitors) are integral parts of the macroscopic system described by the magnetic entropy. In this point of view the variation of the interaction energy $\Phi_{\text {int }}$ vanishes because the transfer of energy from the ohmic transformer to the plasma is internal to the macroscopic system and there is no energy exchange with the external world.

When the Grad-Shafranov form of the current density is introduced in the integral expressing the vanishing of the time derivative of the magnetic entropy one finds strict conditions on the functions $F(\psi)=R B_{\phi}$ and $p(\psi)$ for the toroidal magnetic field and for the pressure. These functions are determined by two separate integro-differential equations (involving the first derivative) that depend on four constants. These equations supplement the Grad-Shafranov equation in the determination of the SME states in toroidal geometry.

\section{Another derivation of the SME equation}

In the derivation of the SME equation for the current density in section 7, the induced electric field, which is a basic ingredient of this equation, has been assumed as generated externally with respect to the macroscopic system described by the magnetic entropy $S_{p}$. It is instructive for a better understanding of the physical aspects of our model, to present a formulation in which the ohmic transformer including the energy source of the primary current, is treated as an integral part of the macroscopic system described by the magnetic entropy. This means that the current of the primary should be included in the functional in addition to the macroscopic current in the plasma. The transformer-plasma interaction is modeled by considering the cylindrical plasma as surrounded by a 
thin conductive shell, with effective radius $r_{s}$, that carries a sheet current density $\vec{j}_{e}$. This current simulates the current in the primary. The current density in the functional (5.4) is the sum of the current density $\vec{j}_{p}$ in the plasma volume and of the time dependent current density $\vec{j}_{e}$ localized in the shell. The time dependent magnetic field $\vec{B}_{e}$ created by $\vec{j}_{e}$ outside the plasma induces the electric field $\vec{E}_{\text {ext }}$ inside the plasma. The field $\vec{E}_{\text {ext }}$ vanishes inside the perfectly conductive shell, while $\vec{E}_{\text {ext }} \neq 0$ with $\nabla \times \vec{E}_{e x t}=0$ in the plasma. The shell is then a surface of discontinuity for $\vec{E}_{e x t}$ and $\partial \vec{B}_{e} / \partial$. The time derivative of the sheet current satisfies the relation

$\frac{4 \pi}{c} \frac{\vec{\partial}_{e}}{\partial}=\delta\left(r-r_{s}\right) \vec{e}_{n} \times \frac{\partial \vec{B}_{e}}{\partial t}$,

where $\vec{e}_{n}$ is the surface unit vector directed outwards. Thus the external time dependence contributes to the time derivative of $S_{p}(5.4)$ according to the relation

$$
\begin{aligned}
& \left(\frac{d S_{p}}{d t}\right)_{e}=\frac{2 \pi}{\tau(\mu c)^{2}}\left(\int_{\Omega} 2 \vec{j}_{p} \frac{\vec{g}_{e}}{\partial} d \Omega-\frac{\mu^{2} c}{4 \pi} \int_{\Omega} \vec{j}_{p} \cdot \frac{\partial \vec{A}_{e}}{\partial} d \Omega-\frac{\mu^{2} c}{4 \pi} \int_{\Omega}\left(\vec{A}_{p}+\vec{A}_{e}\right) \cdot \frac{\vec{\rho}_{e}}{\partial} d \Omega\right)= \\
& \frac{2 \pi}{\tau(\mu c)^{2}}\left(\frac{c}{2 \pi} \int_{S} \vec{j}_{p} \cdot d \vec{S} \times \frac{\partial \vec{B}_{e}}{\partial t}+\frac{(\mu c)^{2}}{4 \pi} \int_{\Omega} \vec{j}_{p} \cdot \vec{E}_{e x t} d \Omega-\left(\frac{\mu c}{4 \pi}\right)^{2} \int_{S} d \vec{S} \times \frac{\partial \vec{B}_{e}}{\partial} \cdot\left(\vec{A}_{p}+\vec{A}_{e}\right)\right),
\end{aligned}
$$

where $S$ is the discontinuity surface, $\vec{A}_{p}$ and $\vec{A}_{e}$ are the vector potentials related to $\vec{j}_{p}$ and $\vec{j}_{e}$ respectively, and

$$
\vec{E}_{e x t}=-\frac{1}{c} \frac{\partial \vec{A}_{e}}{\partial t}, \quad d \vec{S}=\vec{e}_{n} d S, \quad d \Omega=d S d r,
$$

It will be shown presently that the first integral in the right hand side of (18.2) can be expressed in terms of the jump across the discontinuity surface in the direction $d \vec{S}$ of the quantity $\vec{E}_{e x t} \times \nabla \times \vec{j}_{p}$.

Let us apply the coordinate-free definition of the curl to the equations (1/c) $\partial \vec{B}_{e} / \partial t=-\nabla \times \vec{E}_{e x t}$ and $(4 \pi / c) \vec{j}_{p}=\nabla \times \vec{B}$. We consider an infinitesimal volume element $\Delta \tau$ with bases of area $d S$ situated at opposite sides of the discontinuity surface $S$ and parallel to it, and with infinitesimal thickness. According to the definition one has

$$
\nabla \times \vec{E}_{\text {ext }}=\frac{1}{\Delta \tau} \oint d \vec{S} \times \vec{E}_{\text {ext }}, \quad \nabla \times \vec{j}_{p}=\frac{1}{\Delta \tau} \oint d \vec{S} \times \vec{j}_{p} .
$$

where the integral is extended to the surface of the volume element. Noting that $\vec{E}_{\text {ext }}$ and $\vec{j}_{p}$ vanish within the conductive shell, that is on the outer side (with respect to $\Omega$ ) of the discontinuity surface, and are different from zero on the inner side, one obtains

$$
\frac{1}{c} \frac{\vec{B}_{e}}{\partial t}=-\frac{1}{\Delta \tau} \oint d \vec{S} \times \vec{E}_{e x t}=\frac{1}{\Delta \tau} d \vec{S} \times \vec{E}_{e x t}, \quad \frac{1}{\Delta \tau} \oint d \vec{S} \times \vec{j}_{p}=-\frac{1}{\Delta \tau} d \vec{S} \times \vec{j}_{p} .
$$

In the infinitesimal vicinity of $S$, on the inner side where $\vec{E}_{\text {ext }} \neq 0$, one can write

$$
\vec{E}_{e x t} \times \nabla \times \vec{j}_{p}=\vec{E}_{e x t} \times \frac{1}{\Delta \tau} \oint d \vec{S} \times \vec{j}_{p} .
$$

This quantity jumps to zero while crossing the surface $S$ from the inner side towards the conductive shell, where $\vec{E}_{\text {ext }}=0$ :

$$
\left\|\vec{E}_{e x t} \times \frac{1}{\Delta \tau} \oint d \vec{S} \times \vec{j}_{p}\right\|_{\text {inn }}^{\text {out }}=-\vec{E}_{\text {ext }} \times \frac{1}{\Delta \tau} \oint d \vec{S} \times \vec{j}_{p}=\vec{E}_{\text {ext }} \times \frac{1}{\Delta \tau} d \vec{S} \times \vec{j}_{p}=
$$


$=\vec{j}_{p} \times \frac{1}{\Delta \tau} d \vec{S} \times \vec{E}_{e x t}=\vec{j}_{p} \times \frac{1}{c} \frac{\partial \vec{B}_{e}}{\partial t}$,

where use has been made of (18.5). Summing up the value (18.6) on the inner side to the jump (18.7), one obtains the value of $\vec{E}_{\text {ext }} \times \nabla \times \vec{j}_{p}$ on the outer side, within the conductive shell, which vanishes. So $\vec{E}_{e x t} \times \nabla \times \vec{j}_{p}=-\vec{j}_{p} \times \frac{1}{c} \frac{\partial \vec{B}_{e}}{\partial t}$.

Applying the divergence theorem to the integral over the volume $\Omega$ enclosed by $S$ one gets (remembering that $\nabla \times \vec{E}_{\text {ext }}=0$ inside $\Omega$ )

$\int_{S} \vec{E}_{e x t} \times \nabla \times \vec{j}_{p} \cdot d \vec{S}=\int_{\Omega} \vec{E}_{e x t} \cdot \nabla^{2} \vec{j}_{p} d \Omega$

Finally, by combining (18.8) and (18.9) we obtain the desired relation

$\int_{\Omega} \vec{E}_{e x t} \cdot \nabla^{2} \vec{j}_{p} d \Omega=\int_{S} \vec{j}_{p} \cdot d \vec{S} \times \frac{1}{c} \frac{\partial \overrightarrow{\mathrm{B}}_{e}}{\partial t}$.

The last surface integral of (18.2) can be treated in the same way as above by replacing $\vec{j}_{p}$ in (18.10) with $\vec{A}_{p}+\vec{A}_{e}$, where $\vec{A}_{p}$ and $\vec{A}_{e}$, similarly to $\vec{j}_{p}$, drop to zero across $S$ and are different from zero on the inner side of $S$. Noting that $\nabla^{2} \vec{A}_{e}=0$ in $\Omega$, one obtains

$\frac{4 \pi}{c} \int_{\Omega} \vec{E}_{e x t} \cdot \vec{j}_{p} d \Omega=-\int_{S}\left(\vec{A}_{p}+\vec{A}_{e}\right) \cdot d \vec{S} \times \frac{1}{c} \frac{\partial \vec{B}_{e}}{\partial t}$.

Introducing (18.10) and (18.11) into (18.2) we obtain the change of the entropy of the plasma due to the interaction with the ohmic transformer

$\left(\frac{d S_{p}}{d t}\right)_{e}=\frac{1}{\tau} \int_{\Omega} \frac{\vec{E}_{e x t}}{\mu^{2}} \cdot\left(\nabla^{2} \vec{j}_{p}+\mu^{2} \vec{j}_{p}\right) d \Omega$.

The total rate of variation of the entropy is the sum of the contributions of the ohmic transformer (18.12) and of the contribution of auxiliary power and non-diffusive losses

$\frac{d S_{p t o t}}{d t}=\left(\frac{d S_{p}}{d t}\right)_{e}+\frac{1}{\tau} \int_{\Omega} p_{E} d \Omega=\frac{1}{\tau} \int_{\Omega}\left(\frac{\vec{E}_{e x t}}{\mu^{2}} \cdot \nabla^{2} \vec{j}_{p}+\vec{E}_{e x t} \cdot \vec{j}_{p}+p_{E}\right) d \Omega$.

If we assume that this relation vanishes locally, i.e. for any small volume element $\Delta \Omega$ in which $\Omega$ can be subdivided, we obtain again the SME equation (7.4):

$\frac{d S_{p}}{d t}=\frac{1}{\tau} \int_{\Delta \Omega}\left(\frac{\vec{E}_{e x t}}{\mu^{2}} \cdot \nabla^{2} \vec{j}_{p}+\vec{E}_{e x t} \cdot \vec{j}_{p}+p_{E}\right) d \Omega=0$.

Let us see what form takes the time derivative of the interaction energy according to this formulation. The time derivative is calculated starting from the expression (5.5) of $\Phi_{\text {int }}$ where $\vec{j}=\vec{j}_{p}+\vec{j}_{e}(t)$ and $\vec{A}=\vec{A}_{p}+\vec{A}_{e}(t)$. One has

$$
\begin{aligned}
& \frac{d \Phi_{\text {int }}}{d t}=-\frac{1}{2 c} \int_{\Omega} \vec{j}_{p} \cdot \frac{\vec{\partial}_{e}}{\partial t} d \Omega-\frac{1}{2 c} \int_{\Omega}\left(\vec{A}_{p}+\vec{A}_{e}\right) \cdot \frac{\vec{\partial}_{e}}{\partial} d \Omega+\frac{\mu^{2}}{4 \pi} \int_{\Omega}\left(\vec{A}_{p}+\vec{A}_{e}\right) \cdot \frac{\partial \vec{A}_{e}}{\partial t} d \Omega= \\
& =\frac{1}{2} \int_{\Omega} \vec{j}_{p} \cdot \vec{E}_{e x t} d \Omega-\frac{1}{8 \pi} \int_{\partial 2}\left(\vec{A}_{p}+\vec{A}_{e}\right) \cdot d \vec{S} \times \frac{\partial \vec{B}_{e}}{\partial}-\frac{\mu^{2} c}{4 \pi} \int_{\Omega}\left(\vec{A}_{p}+\vec{A}_{e}\right) \cdot \vec{E}_{e x t} d \Omega .
\end{aligned}
$$

Applying the relation (18.11) this equality becomes

$$
\frac{d \Phi_{\mathrm{int}}}{d t}=\int_{\Omega}\left(\vec{j}_{p}-\frac{\mu^{2} c}{4 \pi} \vec{A}_{p}\right) \cdot \vec{E}_{e x t} d \Omega-\frac{\mu c^{2}}{4 \pi} \int_{\Omega} \vec{E}_{e x t} \cdot \vec{A}_{e} d \Omega .
$$


But the minimum principle for the poloidal magnetic energy tells us (section 17, eq.(17.5)) that $\mu^{2} \rightarrow 0$ and $\vec{j}_{p} \rightarrow\left(\mu^{2} c / 4 \pi\right) \vec{A}_{p}$, from which follows that $d \Phi_{\text {int }} / d t \rightarrow 0$.

This result can be understood considering that $\Phi_{\text {int }}$ describes the interaction of the macroscopic system with the external world. But in the present formulation the ohmic transformer should be thought as part of the macroscopic system together with the energy source of the primary current (for instance, a battery of capacitors). At the contrary, in the formulation of section 7 and section 9 the induced electric field was applied to the macroscopic system externally and this is reflected in the fact that $d \Phi_{\text {int }} / d t$, given by (8.3) or (9.8), describes the Poynting flux of electromagnetic energy related to $\vec{E}_{\text {ext }}$ across the surface enclosing the macroscopic (collective) system.

\section{Conditions on the toroidal equilibrium of the tokamak}

The toroidal axis-symmetric equilibrium is described by the Grad-Shafranov equation

$j_{\phi}=\frac{C}{4 \pi} R \nabla \cdot\left(R^{-2} \nabla \psi\right)$

where

$j_{\phi}=-\frac{c R}{4 \pi}\left(\frac{\left(F^{2}(\psi)\right)}{2 R^{2}}+4 \pi p(\psi)\right)$.

Here $p(\psi)$ is the pressure, $F(\psi)=R B_{\phi}$ and the poloidal flux $\psi$ is related in symmetry coordinates to the magnetic field by $\vec{B}=(F(\psi) / R) \vec{e}_{\phi}+\left(\vec{e}_{\phi} / R\right) \times \nabla \psi$.

In this section it will be shown with the help of the SME condition (18.14) and of the minimum plasma energy principle discussed in section 16, that the functions $F(\psi)$ and $p(\psi)$ are determined by two separate integro-differential equations (involving the first derivative) that depend on four constants. We shall use the relation

$I(\psi)=\frac{c}{8 \pi^{2}} \int_{S(\psi)} \frac{\nabla \psi \cdot d \vec{S}}{R^{2}}$,

where $I(\psi)$ is the current flowing inside the magnetic surface $S(\psi)$. Indeed, denoting with $d A$ the element of area of the cross section of a surface $\psi=$ const and with $d \Omega=2 \pi R d A$ the volume element, one has

$I(\psi)=\int_{A(\psi)} j_{\phi} d A=\int_{\Omega} j_{\phi} \frac{d \Omega}{2 \pi R}=\frac{c}{8 \pi^{2}} \int_{\Omega} \nabla \cdot \frac{\nabla \psi}{R^{2}} d \Omega=\frac{c}{8 \pi^{2}} \int_{S(\psi)} \frac{\nabla \psi \cdot d \vec{S}}{R^{2}}$.

We now proceed by applying the Green theorem to the SME condition (18.14) where $\Delta \Omega$ is a toroidal shell comprised between two magnetic surfaces:

$\frac{1}{\mu^{2}} \int_{S_{e}}\left(E_{e x t} \nabla j_{\phi}-j_{\phi} \nabla E_{e x t}\right) \cdot d \vec{S}+\frac{c E_{0} R_{0}}{4 \pi} \int_{S_{e}} \frac{\nabla \psi \cdot d \vec{S}}{R^{2}}+\int_{\Omega_{e}} p_{E} d \Omega=$
$\frac{1}{\mu^{2}} \int_{S_{i}}\left(E_{e x t} \nabla j_{\phi}-j_{\phi} \nabla E_{e x t}\right) \cdot d \vec{S}+\frac{c E_{0} R_{0}}{4 \pi} \int_{S_{i}} \frac{\nabla \psi \cdot d \vec{S}}{R^{2}}+\int_{\Omega_{i}} p_{E} d \Omega$.

Here $d \vec{S}=d S \nabla \psi / \nabla \psi \mid$, and $S_{e}, S_{i}$ are the outboard and inboard magnetic surfaces delimiting the toroidal shell $\Delta \Omega, \Omega_{e}, \Omega_{i}$ are the volumes enclosed by $S_{e}$ and $S_{i}$ respectively and $E_{\text {ext }}=E_{0} R_{0} / R$ where $E_{0}, R_{0}$ are values on the minor axis. In order to satisfy the equality (19.5) the two members must 
be independent of $\psi$, that is to say, for any surface enclosing the volume $\Omega(\psi)$, the following relation must hold:

$\int_{S(\psi)}\left(E_{e x t} \nabla j_{\phi}-j_{\phi} \nabla E_{e x t}\right) \cdot d \vec{S}+\frac{c E_{0} R_{0} \mu^{2}}{4 \pi} \int_{S(\psi)} \frac{\nabla \psi \cdot d \vec{S}}{R^{2}}+\mu^{2} \int_{\Omega(\psi)} p_{E} d \Omega=D$,

where $D$ is independent of $\psi$. After expressing $j_{\phi}$ with (19.2) (we neglect the contribution of noninductive currents) the equality (19.6) becomes a relation between $F(\psi)$ and $p(\psi)$ :

$\frac{2 c p(\psi)}{\pi I(\psi)} \int_{S(\psi)} \frac{\vec{e}_{R} \cdot d \vec{S}}{R}+\frac{c p^{\prime \prime}(\psi)}{\pi I(\psi)} \int_{S(\psi)} \nabla \psi \cdot d \vec{S}+\left(F^{2}(\psi)\right)^{\prime}=$

$2 \mu^{2}+\frac{\mu^{2}}{\pi E_{0} R_{0} I(\psi)} \int_{\Omega(\psi)} p_{E} d \Omega-\frac{D}{\pi E_{0} R_{0} I(\psi)}$.

Introducing two functions $k_{1}(\psi)$ and $k_{2}(\psi)$ (arbitrary a priori) one can split the relation (19.7) into separate equations for $F(\psi)$ and $p(\psi)$ :

$2 c p(\psi) \int_{S(\psi)} \frac{\vec{e}_{R} \cdot d \vec{S}}{R}+c p^{\prime \prime}(\psi) \int \nabla \psi \cdot d \vec{S}=\frac{k_{1}(\psi) \mu^{2}}{E_{0} R_{0}} \int_{\Omega(\psi)} p_{E} d \Omega-\frac{k_{2}(\psi) D}{E_{0} R_{0}}$,

$\left(F^{2}(\psi)\right)^{\prime}=2 \mu^{2}+\frac{\left(1-k_{1}(\psi)\right) \mu^{2}}{\pi I(\psi) E_{0} R_{0}} \int_{\Omega(\psi)} p_{E} d \Omega-\frac{1-k_{2}(\psi)}{\pi I(\psi) E_{0} R_{0}} D$.

In a situation where the cross-section of the magnetic surface is up-down symmetric the first integral of (19.8a) vanishes. In this case the first integrals of eqs. (19.8) take on the form

$p(\psi)-p_{s}=\frac{\mu^{2}}{c E_{0} R_{0}} \int_{0}^{\psi} \frac{k_{1}(\psi) d \psi}{\int_{S(\psi)} \nabla \psi \cdot d \vec{S}} \int_{\Omega(\psi)} p_{E} d V-\frac{D}{c E_{0} R_{0}} \int_{0}^{\psi} \frac{k_{2}(\psi) d \psi}{\int_{S(\psi)} \nabla \psi \cdot d \vec{S}}$,
$\left(F^{2}(\psi)\right)-\left(F^{2}\right)_{s}=2 \mu^{2} \psi+\frac{\mu^{2}}{\pi E_{0} R_{0}} \int_{0}^{\psi} \frac{\left(1-k_{1}(\psi)\right) d \psi}{I(\psi)} \int_{\Omega(\psi)} p_{E} d V+\frac{D}{\pi E_{0} R_{0}} \int_{0}^{\psi} \frac{\left(k_{2}(\psi)-1\right) d \psi}{I(\psi)}$.

Here $p_{s}^{\prime}$ and $\left(F^{2}\right)_{s}^{\prime}$ are values at $r=s a$ (where we have taken $\psi=0$ ).

Since the functions $k_{1}(\psi)$ and $k_{2}(\psi)$ depend only on the poloidal flux and not explicitly on the aspect ratio, they will maintain their form independently of the aspect ratio and then we can determine them in the large aspect ratio limit. It is easy to write down the equations (19.7) in this limit:

$p^{\prime}(\psi(x))=\frac{d p}{d x} \frac{d x}{d \psi}-\frac{(\mu s a)^{2}}{c E R} \int_{1}^{x} \frac{k_{1} d x}{x} \int_{0}^{x} p_{E} x d x-\frac{D}{4 \pi^{2} R^{2} c E} \int_{1}^{x} \frac{k_{2} d x}{x}+p_{s}^{\prime}$,
$\left(F^{2}\right)^{\prime}=\left(F^{2}\right)_{s}^{\prime}+\mu^{2} \psi+\frac{8 \pi R(\mu s a)^{2}}{c E} \int_{1}^{x} \frac{1-k_{1}}{x} d x \int_{0}^{x} p_{E} x d x+\frac{2 D}{\pi c E} \int_{1}^{x} \frac{\left(k_{2}-1\right) d x}{x}$.

By introducing these expressions into (19.2) we obtain the current density in the large aspect ratio limit

$j_{\phi}=-\frac{\mu^{2} c}{4 \pi R} \psi-\frac{(\mu s a)^{2}}{E} \int_{1}^{x} \frac{d x}{x} \int_{0}^{x} p_{E} x d x+\frac{D}{4 \pi^{2} E R} \ln x-\frac{c\left(F^{2}\right)_{s}^{\prime}}{8 \pi R}-c R p_{s}^{\prime}$.

Comparing this expression with (16.3) one gets 
$D_{0}=\frac{D}{4 \pi^{2} E R}, \quad D_{1}=-\frac{c\left(F^{2}\right)_{s}^{\prime}}{8 \pi R}-c R p_{s}^{\prime}$.

We express (19.10a) in terms of $D_{0}$ :

$p^{\prime}(\psi(x))=\frac{(\mu s a)^{2}}{c E R} \int_{1}^{x} \frac{k_{1} d x}{x} \int_{0}^{x} p_{E} x d x-\frac{D_{0}}{R c} \int_{1}^{x} \frac{k_{2} d x}{x}+p_{s}^{\prime}$.

Comparing this expression with the expression (16.10) obtained from the minimum plasma energy principle we observe that $k_{1}$ and $k_{2}$ must be constant. Moreover, by equating the coefficients of the first two terms in the right hand of (16.10) and (19.13) one obtains

$k_{1}=\frac{\eta_{1} \mu^{2} c^{2}}{6 \pi}=k_{2}$,

and by equating the last terms

$\eta_{2}=\frac{12 \pi^{2} R^{2}}{c^{2} \mu^{2}}\left(p_{s}^{\prime}+\frac{D_{1} k_{1}}{c R}\right)$.

We conclude that the functions $F(\psi)$ and $p(\psi)$ are determined by the two separate equations (19.9a) and (19.9b) that depend on the four constants $D, p_{s}^{\prime},\left(F^{2}\right)_{s}^{\prime}$ and $k_{1}=k_{2}=$ const.

The toroidal equilibrium of the SME state is described by the Grad-Shafranov equation (19.1) supplemented by the two conditions (19.9).

\section{CHAPTER 6: Comparisons between theory and experiment}

Summary: In this chapter the theoretical profiles of the SME states in tokamaks are compared with the observations.

The first comparisons were performed with the data of ohmic discharges in TCV [19]. The location of the $q=1$ surface (identified with the inversion radius) and the ratio of the average temperature to the temperature on the magnetic axis is controlled in this machine by the parameter $<j>/ j_{0} q_{0}(<j>$ is the cross-sectionally averaged current density, $j_{0}, q_{o}$ are values on the magnetic axis). In the entire range of quasi-stationary ohmic conditions the observed dependencies on this parameter in the confinement region were found in excellent agreement with the theoretical predictions provided that $\mu s a<<1$. The observed electron pressure profiles are consistent with those derived from the minimum plasma energy principle and characterized by the convexity parameter $K<1$.

Comparisons of the temperature and pressure profiles, as well as of the thermal conductivity given by eqs.(7.6) and (7.7), were performed in ECR discharges in FTU. Successively the validity of the theory has been scrutinized in a number of shots in JET and TS under different conditions of plasma regime and heating methods, taking into account the possible existence of non-inductive currents.

The whole of these comparisons confirms the robustness of the SME profiles in the absence of strong non-inductive currents or of the effects of rapid ramps-up of the current. 


\section{Comparisons with the experiment; case of ohmically relaxed currents}

20.1-Current density, temperature and pressure profiles

The next two sections are devoted to the comparison of the predictions of the SME theory with the observations. The presentation is a summary of the results contained in previous papers on comparisons in TCV [19], in FTU [20], in JET and in TS [21]. We refer the reader to these papers for the details.

The first evidence on the reliability of the theory has been collected by Weisen [19] on ohmic quasistationary discharges in TCV $\left(B_{T}=1.5 T, R_{0}=0.88 \mathrm{~m}, a<0.25 \mathrm{~m}\right)$. The discharges concerned limited and diverted shots with elongations in the range $-0.5-0.72$ and plasma currents in the range $0.1-1.0 \mathrm{MA}$. In the entire range of quasi-stationary ohmic conditions it was observed that the sawtooth inversion radius and the electron temperature in the confinement region depend solely on the parameter $\left\langle j>/ j_{0} q_{0}\right.$ where $\langle j\rangle$ is the cross-sectionally averaged current density and $q_{0} j_{0}=\left(c B_{0} / 4 \pi R_{0}\right)\left(k_{e}^{2}+1\right) / k_{e}$. Here $k_{e}$ is the elongation and the subscript denotes quantities on the magnetic axis. In cylindrical geometry the solution to equation (7.4) (with $p_{E}=0$ and the boundary conditions $j(\lambda a)=\hat{j}, j(a)=0$ ) are expressed in term of the Bessel functions

$j(r)=\frac{\hat{j}}{D}\left(J_{0}(\mu a) Y_{0}(\mu r)-Y_{0}(\mu a) J_{0}(\mu r)\right)$,

where $D=J_{0}(\mu a) Y_{0}(\mu r)-Y_{0}(\mu a) J_{0}(\mu r)$.

Here and henceforth caps denote quantities on the $q=1$ surface (the inversion radius is identified with the $q=1$ surface). In the sawtooth region $r<\lambda a$ the safety factor $q(r)$ is modeled as in section 9 .

The solution (20.1) entails the following relation for the experimentally relevant parameter $<j>/ j_{0} q_{0}$ :

$\frac{<j>}{j_{0} q_{0}}=\lambda^{2}-\frac{2 q_{0}}{\mu^{2} D}\left(\frac{2}{\lambda \mu a}+\lambda \Lambda\right)$

where $\Lambda=J_{0}(\mu a) Y_{1}(\lambda \mu a)-Y_{0}(\mu a) J_{1}(\lambda \mu a)$.

The diamagnetic and paramagnetic corrections to the toroidal field are small [18] and are neglected.

In fig. 2 the relation (20.3) is compared with the experimental data for $q_{0}$ in the range $0.8-1.0$ Agreement is found for $\mu a<1$ as expected from the theoretical considerations of section 17 , and the results become practically indistinguishable for $\mu a \rightarrow 0$. One concludes that the poloidal magnetic configuration is essentially determined by $\lambda$ or, alternatively. by $<j>/ j_{0} q_{0}$.

In a stationary ohmically relaxed discharge the current density profile is related to the temperature, pressure and density profiles by the Ohm law. When only Spitzer conductivity is considered one has the relations

$\frac{n_{e}}{\hat{n}_{e}}=\frac{p_{e} / \hat{p}_{e}}{(j / \hat{j})^{2 / 3}}=\frac{p_{e} / \hat{p}_{e}}{T_{e} / \hat{T}_{e}}$.

The effect of neoclassical corrections has been discussed [19] and found to be small. In fig. 3 the theoretical temperature, pressure and temperature experimental profiles are presented together with the theoretical profiles (neoclassical corrections are taken into account). Note that in the figures the 
quantities on the $q=1$ surface are referred to by the subscript 1 , whereas in the text the diacritical mark $\wedge$ has been used.

Figure 2. Sawtooth inversion radii from $X$ ray tomography in TCV (symbols) and theoretical predictions for the $\mathrm{q}=1$ surface (bold curves) for three assumed values of $q_{0}$ (from Minardi E.; Weisen H. Nucl. Fusion 2001, 41, 113-130) (with permission).

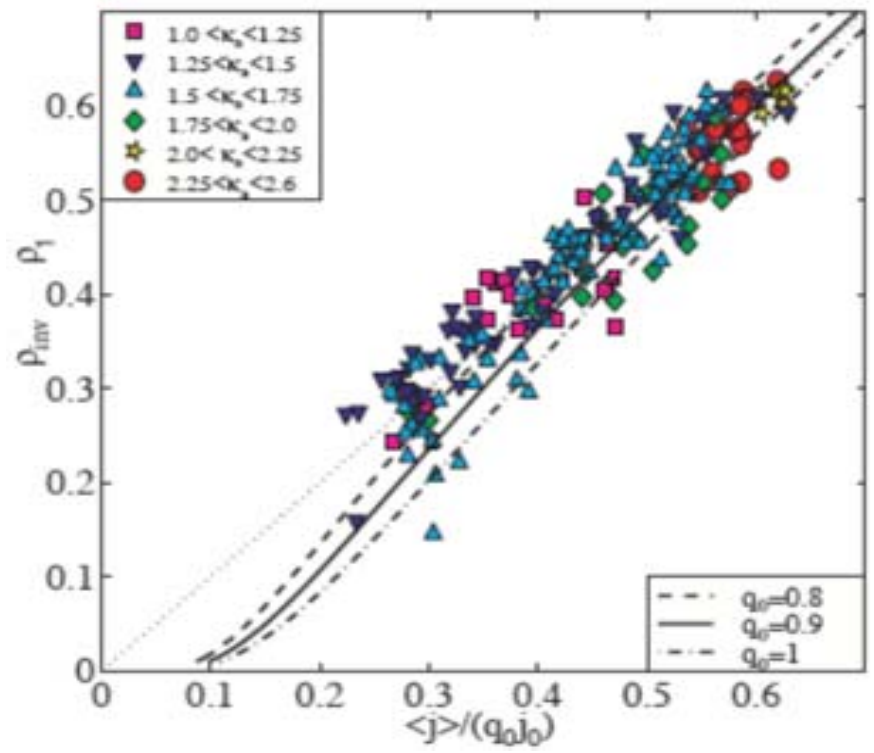

Figure 3. Example of experimental electron pressure (plus sign), temperature (crosses) and density (circles) profiles from Thomson scattering in TCV, together with theoretical profiles. The solid curves are obtained using neoclassical Ohm's low and the dashed curves using Spitzer resistivity alone (from Minardi E.; Weisen H. Nucl. Fusion 2001, 41, 113130) (with permission).

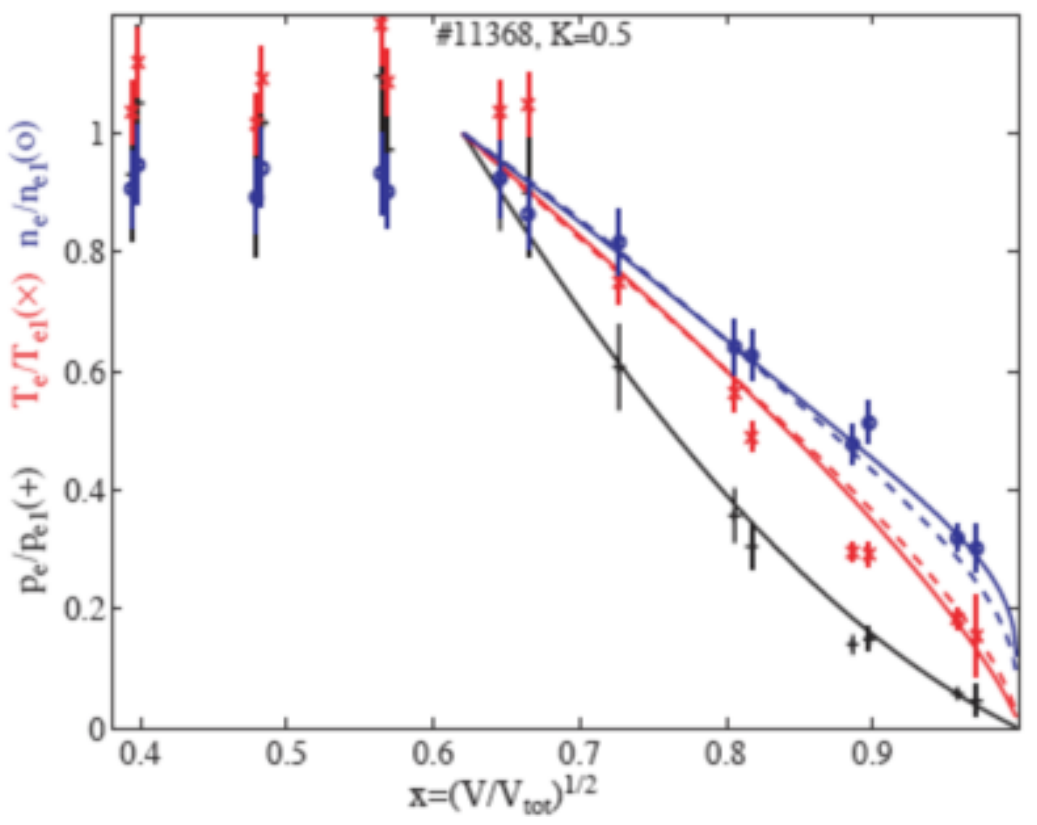


Figure 4. Widths of clipped electron temperature profiles: diamonds, experiment; dots, theory with neoclassical Ohm’s law (from Minardi E.; Weisen H. Nucl. Fusion 2001, 41, 113-130) (with permission).

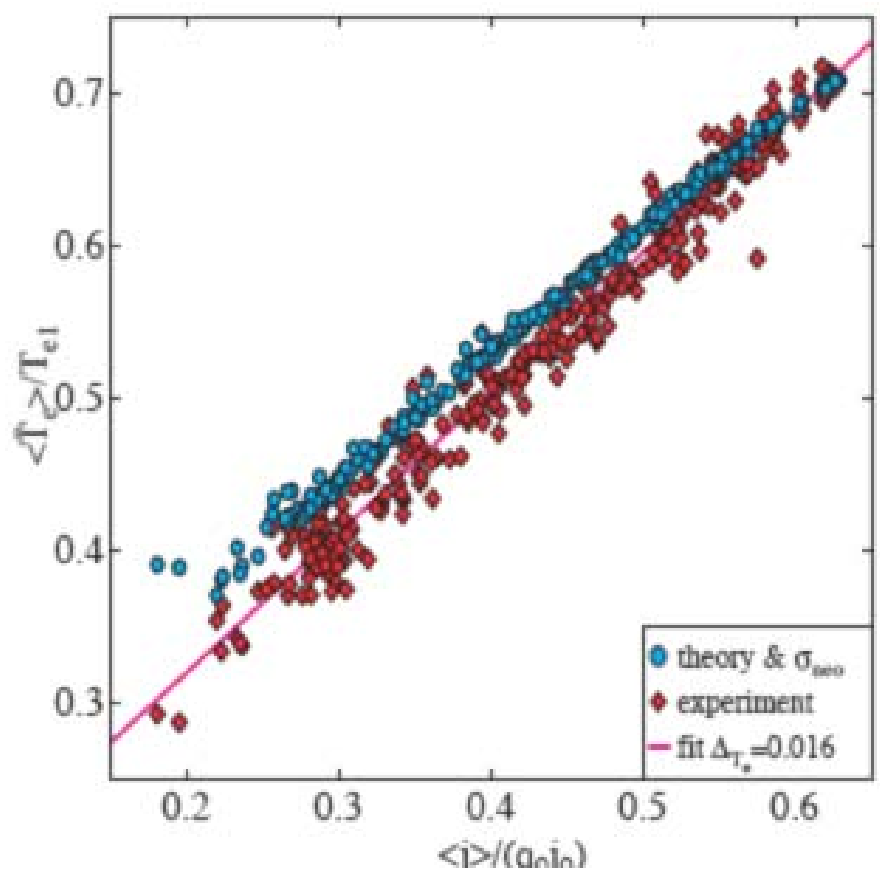

Figure 5. Experimental pressure profile convexity parameter $\mathrm{K}$ for different elongations $\kappa_{a}$ (from Minardi E.; Weisen H. Nucl. Fusion 2001, 41, 113-130) (with permission).

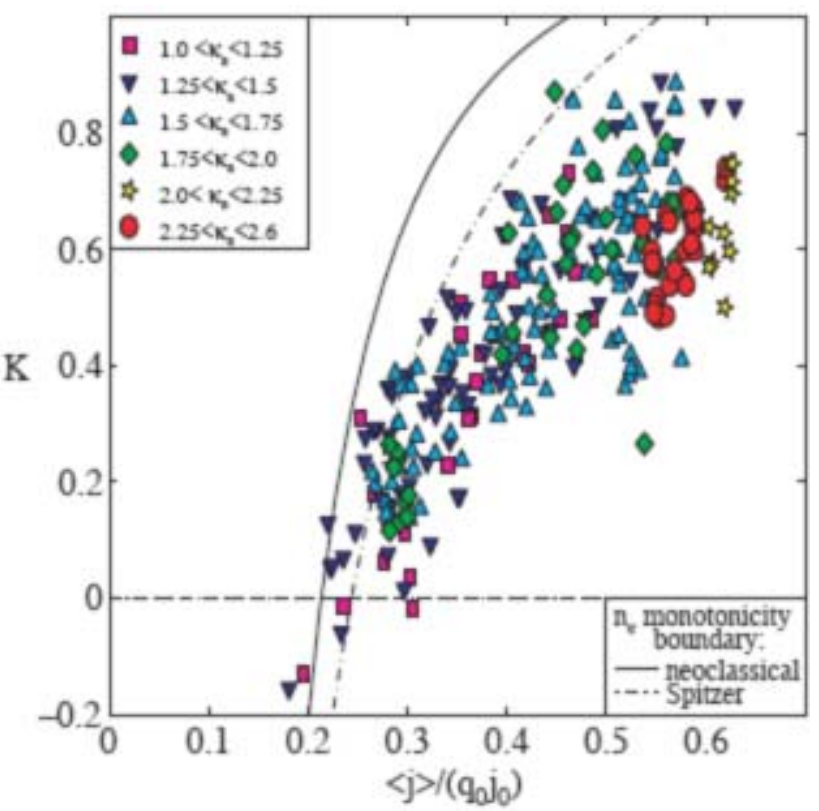

During the sawtooth cycle, core plasma temperatures rise and flatten periodically, while temperatures at and beyond the inversion radius experience only small variations. This effect introduces a significant amount of scatter in the data [22-23]. Only the confinement region may be considered as ohmically relaxed and close to steady state. In order to take into account this effect in the 
comparison with the theoretical model "clipped" profile widths were introduced in the data of fig. 4 such as $<\bar{T}_{e} / T_{e}>$ where $\bar{T}_{e}=\hat{T}$ for $r<\lambda a$ and $\bar{T}_{e}=T$ for $r>\lambda a$.

Another significant subject of experimental investigation is the value of the convexity parameter $K$ introduced in section 16, which should be lower than one in order that the plasma thermal energy is at a minimum. The experimentally measured widths $\left\langle\bar{p}_{e} / \hat{p}_{e}\right\rangle$, which are strongly correlated to $<j>/ j_{0} q_{0}$, are associated with the values of $K$ as shown in fig. 5 . With few exceptions, attributable to experimental uncertainties, the experimental values of $K$ do indeed lie in the interval $0<K<1$, as predicted at the end of section 16 .

Once the parameter $K$ is known, the pressure profile is determined by (16.14) and (16.17) and the density profile follows from the ohmic relaxation (20.5).

Alternatively, canonical density profiles are also provided by turbulent equipartition (TEP) theories according to the relation $n=\hat{n} q^{-\eta}$ where $0<\eta<1$ [24]. For a comparison between the two approaches in the ohmic case see a paper by Weisen and Minardi [25]. It turns out that the relation above for the density is also often verified in discharges with auxiliary heating and in the absence of ohmic relaxation.

\section{2-Thermal conductivity}

In cylindrical geometry the expression (7.3) for the heat flux in the relaxed region $\lambda s a<r<s a$ takes on the form

$q_{h} S=-\frac{E_{e x t}}{\mu^{2}}\left(\frac{d j}{d r}-\frac{C}{r}\right)$,

where $S=4 \pi^{2} r R$ and $C$ is a constant. Thus, recalling (7.2), the power balance of the electrons becomes

$q_{h} S=-\frac{E_{\text {ext }}}{\mu^{2}}\left(\frac{d j}{d r}-\frac{C}{r}\right)=4 \pi^{2} R \int_{0}^{r}\left(E_{\text {ext }} j+p_{E}\right) r d r$,

where $C$ is determined by continuity of the heat flux across the surface $\hat{q}=1$ (we note that this implies at the same time the continuity of the poloidal magnetic field which is proportional to $\int j r d r$ ).

Modelling the magnetic configuration in the region $r<\lambda s a$ according to the expression $q(x)=q_{0}+\left(1-q_{0}\right)(r / \lambda)^{2}$ for the safety factor, one obtains $\hat{I}=\pi j_{0} q_{0}(\lambda s a)^{2}, j_{0}=c B / 2 \pi R q_{0}, \hat{j}=j_{0} q_{0}^{2}$ respectively for the total current along the cylinder with radius $r=\lambda$ sa, for the current density on axis and on the surface $\hat{q}=1$. Then $C=j_{0} q_{0} \beta$ where

$\beta \equiv \frac{(\mu s \lambda)^{2}}{2}\left(1-\left(\frac{\hat{v}}{\mu}\right)^{2}+\frac{2 q_{0}}{\lambda^{2}} \int_{0}^{\lambda} \frac{p_{E}}{E_{e x t} \hat{j}} x d x\right)$.

Here $\hat{v}=-(4 \pi R / c)^{2}(d j / d \psi)_{\lambda s a}$.

Putting

$h(r)=1-\frac{j_{0} q_{0} \beta}{r c d j / d r}$,

the effective thermal conductivity (7.7) becomes 
$n_{e} \chi_{\text {eff }}=-\frac{q_{h}}{d T / d r}=E_{e x t} \frac{d j / d r}{d T / d r} \frac{h(r)}{\mu^{2}}$.

In the case of the ohmically relaxed current, i.e. $j=E_{\text {ext }} T^{3 / 2} A\left(Z_{\text {eff }}\right)$ with Spitzer resistivity $\left(A\left(Z_{\text {eff }}\right)=1.9745 \cdot 10^{-31}\right.$ in CGS units, corresponding to $\ln \Lambda=17$ ) and $Z_{\text {eff }}$ uniform, one has

$n_{e} \chi_{\text {eff }}=\frac{3}{2} \frac{E_{e x t}^{2}}{A\left(Z_{\text {eff }}\right)} T^{3 / 2} \frac{h(r)}{\mu^{2}}=\frac{3}{2} \frac{E_{\text {ext }} j}{T} \frac{h(r)}{\mu^{2}}$,

where $h(r) / \mu^{2}$ is independent of $\mu^{2}$.

The form (20.11) of the diffusivity has been tested in ECR discharges in FTU (see Table II for the main parameters of the discharges). In shot 18290 the whole EC power is absorbed at $r / a=0.45$, whereas in shot 18281 the absorbed power is shared in two separate layers ( $r / a=0.3$ and $r / a=0.63$ ) using poloidal steering of beams (see fig.6). The very localized power deposition induces a step-like behavior of $\chi_{\text {eff }}$ if and only if the $T(r)$ profile is locally stiff. Now $T(r) \approx j(r)^{2 / 3}$ where $j$ is a solution of the SME equation, which is insensitive to the power deposition for $\mu$ sa sufficiently small ( $\mu$ sa $<0.20$, see Table III). The step-like behavior of $\chi_{\text {eff }}$ in comparison with the experimental one is shown in fig.7. This behavior follows from a critical balance between the two terms of the function $h(r)$. Expressing $h(r)$ as function of the temperature gradient $R / L_{e}$, where $L_{e}^{-1}=-(1 / T) d T / d r$, one finds a step-like behavior (fig.8) at a critical gradient associated with a location of the ECR power deposition. When the heat flux is expressed in terms of the parameter $s_{h} / q$, as in (7.6), one finds that $s_{h} / q \approx-\beta$ at this location, where $\beta<0$ depends only on the magnetic configuration according to (20.8) and determines the term of $h(r)$ associated with the inward part of the heat flux.

Figure 6. Net power density on electrons, without ohmic component, calculated with EVITA power balance code. FTU18290 in ECH phase (red dashes) and in ohmic one (black dashes); FTU18281 in ECH phase (black solid) (from Minardi E.;Lazzaro E.; Sozzi C.; Cirant S. Nucl. Fusion 2003, 43, 369-384) (with permission).

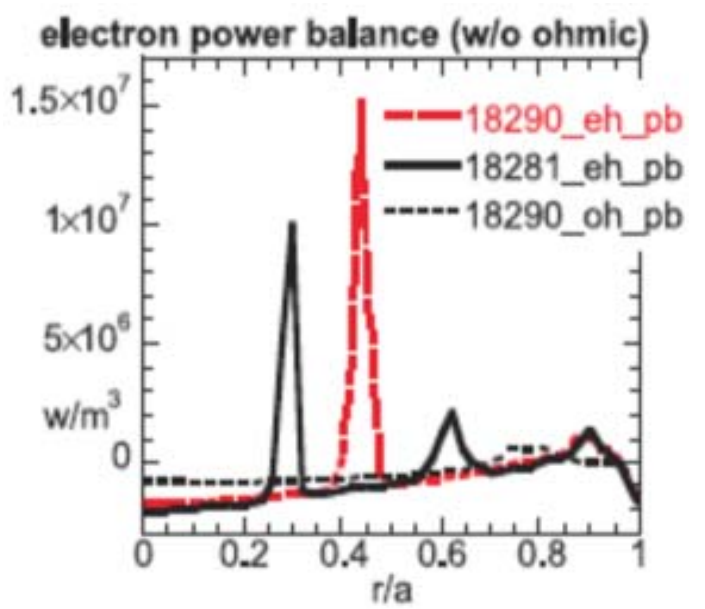

The current density and pressure profiles of shot 18290 predicted by the SME equations (7.4) and (16.14) (with the conditions at the boundaries taken from experiment) are compared in figs.9a and $9 \mathrm{~b}$ with the profile reconstruction based on the experimental data. 
Figure 7. Symbols “+” and “ $\times$ ”: from EVITA interpretative power balance code; lines: from theory. $B=5.7 T$ for FTU18281 and $B=5.8 T$ for FTU18290; $n_{e(\text { line) }}=0.8 \times 1010^{20} \mathrm{~m}^{-3}, I_{p}=0.4 \mathrm{MA}$ for both shots (from Minardi E.,;Lazzaro E.; Sozzi C.;

Cirant S. Nucl. Fusion 2003, 43, 369-384) (with permission).

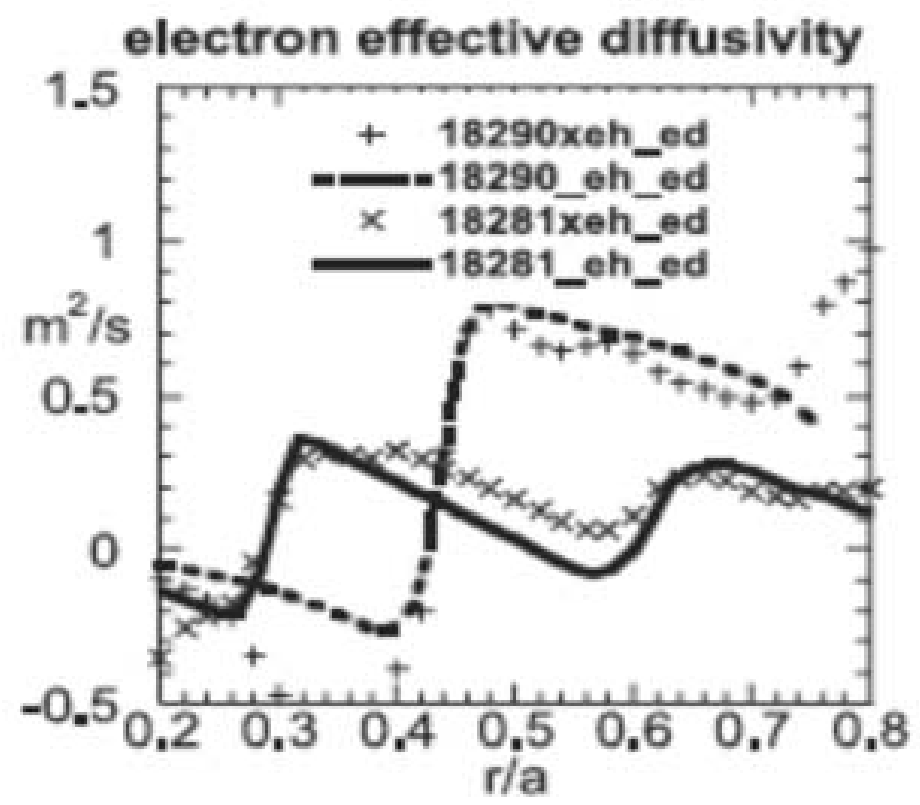

Figure 8. The $h(r)$ function (see eqs.(20.8) and (20.9)) against normalized electron temperature gradient length for shot FTU18290 (symbols: experiment; line: theory (from Minardi E.; Lazzaro E.; Sozzi C.; Cirant S. Nucl. Fusion 2003, 43, 369-384) (with permission).

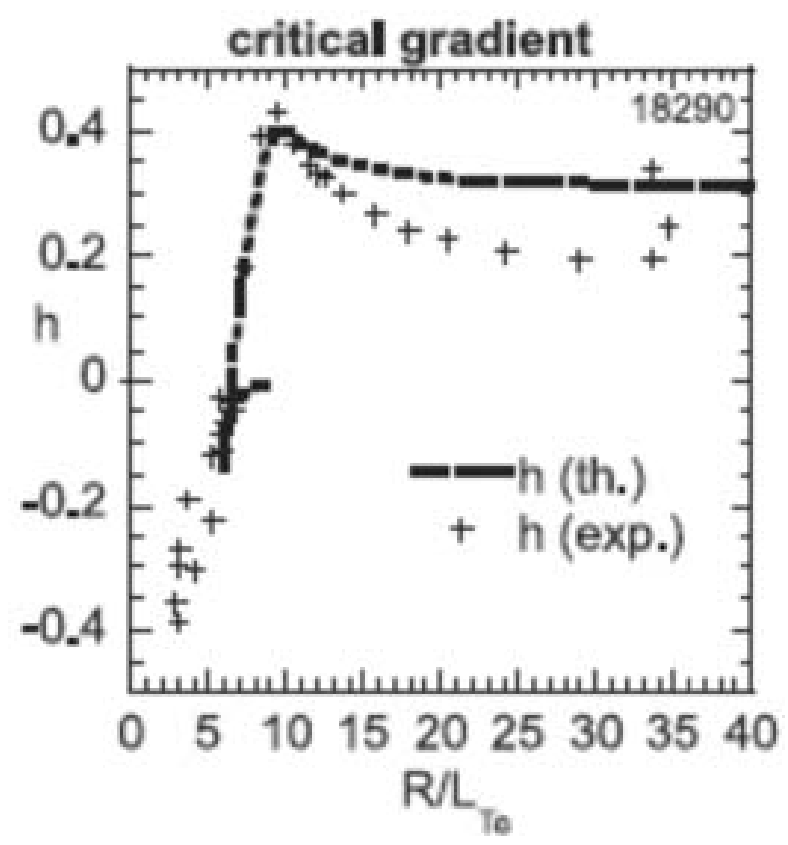


Figure 9. Experimental (symbols) and theoretical (line) current density (a) and normalized pressure profiles (b) in FTU 1890 discharge measured at 1.1s (from Minardi E.,; Sozzi C.; Mantica P. Nucl. Fusion 2008, 48, 045001) (with permission).
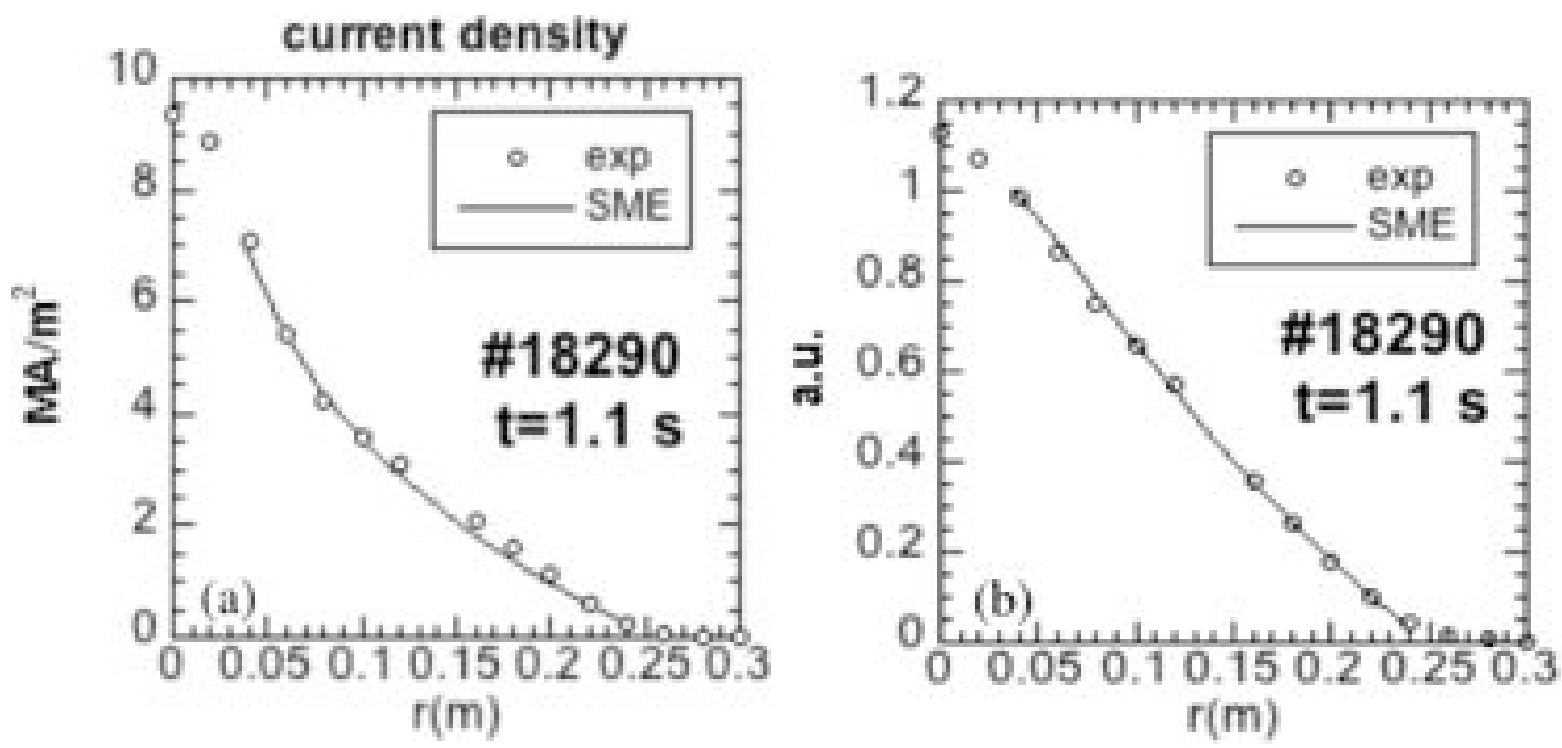

\section{L- and H- discharges and the effect of non-inductive currents}

The reliability of the SME predictions has been analyzed in a variety of tokamak discharges whose characteristic parameters are listed in Table II. The experimental current density profiles of JET are obtained solving the MHD equilibrium with EFIT, either using only magnetic measurements or when available the MSE measurements. This method implies that the (discrete) experimental measurements are given as constraints to the equilibrium solver, which then yields smooth current density profiles as the solution of the equilibrium equations that best fits the given experimental constraints. The form of $p_{E}$ in eq.(7.4) and of its components (auxiliary power, radiation losses, electron-ion energy transfer) as well as the profiles of the non inductive components of the current density are obtained by interpretative transport simulations with standard transport codes . In FTU and TS the profiles of the safety factor (which determines the current density, see eq. (7.5)) and of the pressure are obtained consistently with the transport simulations (JETTO, ASTRA, CRONOS, EVITA). This process produces the radial profile of the current density and of the pressure to be compared with those predicted by the SME equations (7.4) and (16.14).

The SME equations are solved in a relaxed zone $s a \lambda \leq r \leq s a$ using for $E$ the experimental value obtained from the loop voltage and taking from experiment the values of $j$ and $p$ at the boundaries. The input $p_{E}$ to (7.4), which gives the net non-ohmic power on electrons, is supplied by the interpretative transport codes.

A geometrical correction for noncircular geometry, even though approximate, is applied to the calculation of all integral quantities (total current, total power, magnetic poloidal flux, safety factor and so on) by expressing the cross-sectional area with the cylindrical coordinates $x=r \cos \theta, y=r k_{e}(r) \sin \theta$, Here $k_{e}$ is the elongation given by the expression $k_{e}(r)=\left(k_{e 1}-k_{e 0}\right) r / a+k_{e 0}$ (see Table II for the values of $k_{e 0}$ and $k_{e 1}$ and the quoted paper [20] for further details). 
Table II. Main parameters of the discharges analyzed in the paper: $I(M A)$, total current; $n_{e 0}$, central electron density in $m^{-3} ; B_{0}(T)$, external toroidal field; $k_{e 0}$ and $k_{e 1}$, elongation parameters (see text); $P_{a}(M W)$, auxiliary power (from Minardi E.; Sozzi C.; Mantica P. Nucl. Fusion 2008, 48, 045001) (with permission).

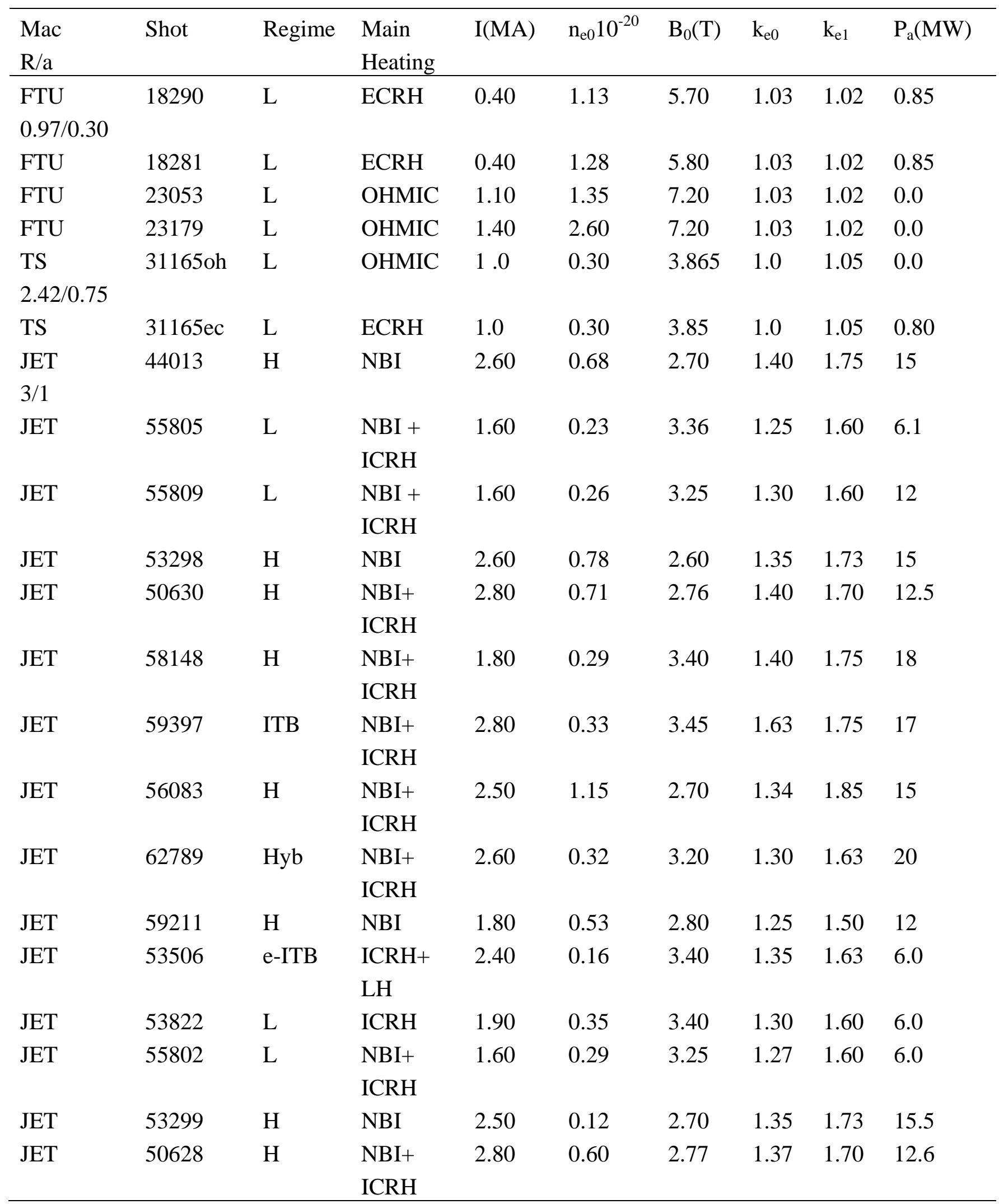


Table III. Meaning of symbols: $a$, minor radius; sa and $s a \lambda$, outer and inner border of the relaxed region respectively; $\mu s a$, indicative value below which the profiles are independent of $\mu s a$; $U(V)$, loop voltage in Volt; $\Delta q$, range of safety factor in the relaxed region; erj and erp. mean square deviations of the current density and pressure with respect to the experimental profiles (from Minardi E.; Sozzi C.; Mantica P. Nucl. Fusion 2008, 48, 045001) (with permission).

\begin{tabular}{lllllllll}
\hline Mac & Shot & $\mu s a$ & $\mathrm{U}(\mathrm{V})$ & $\mathrm{s}$ & $\lambda$ & $\Delta q$ & errj & errp \\
\hline FTU & 18290 & 0.20 & 1.0 & 0.73 & 0.17 & $1-3.94$ & 0.042 & 0.022 \\
FTU & 18281 & 0.03 & 1.0 & 0.75 & 0.15 & $1-3.93$ & 0.071 & 0.035 \\
FTU & 23053 & 0.15 & 1.73 & 0.80 & 0.55 & $1-2.15$ & 0.086 & 0.023 \\
FTU & 23179 & 0.03 & 2.60 & 0.80 & 0.62 & $1-1.72$ & 0.033 & 0.034 \\
TS & $31165 \mathrm{oh}$ & 0.01 & 0.80 & 0.93 & 0.20 & $1-4.13$ & 0.068 & 0.011 \\
TS & $31165 \mathrm{ec}$ & 0.10 & 0.55 & 0.93 & 0.22 & $1-4.09$ & 0.070 & 0.043 \\
JET & 44013 & 0.30 & 0.20 & 0.75 & 0.44 & $1-2.15$ & 0.031 & 0.027 \\
JET & 55805 & 0.05 & 0.33 & 0.90 & 0.12 & $1-5.88$ & 0.039 & 0.086 \\
JET & 55809 & 0.01 & 0.40 & 0.75 & 0.13 & $1-3.92$ & 0.076 & 0.063 \\
JET & 53298 & 0.01 & 0.15 & 0.80 & 0.40 & $1-2.20$ & 0.058 & 0.020 \\
JET & 50630 & 1.70 & 0.35 & 0.90 & 0.16 & $1.03-2.78$ & 0.079 & 0.040 \\
JET & 58148 & 0.10 & 0.20 & 0.90 & 0.40 & $2.17-5.36$ & 0.035 & 0.040 \\
JET & 59397 & 0.65 & 0.60 & 0.95 & 0.44 & $1.71-4.33$ & 0.039 & 0.026 \\
JET & 56083 & 0.25 & 0.25 & 0.80 & 0.51 & $1-2.11$ & 0.021 & 0.036 \\
JET & 62789 & 0.25 & 0.70 & 0.70 & 0.35 & $1.02-2.60$ & 0.075 & 0.036 \\
JET & 59211 & 0.10 & 0.20 & 0.80 & 0.19 & $1.25-3.93$ & 0.026 & 0.024 \\
JET & 53506 & 0.10 & 0.20 & 0.90 & 0.44 & $1.76-4.20$ & 0.043 & 0.043 \\
JET & 53822 & 0.05 & 0.40 & 0.70 & 0.23 & $1-3.48$ & 0.077 & 0.039 \\
JET & 55802 & 0.40 & 0.30 & 0.75 & 0.15 & $1-3.55$ & 0.030 & 0.060 \\
JET & 53299 & 0.05 & 0.20 & 0.80 & 0.45 & $1-2.21$ & 0.042 & 0.044 \\
JET & 50628 & 0.10 & 0.35 & 0.95 & 0.20 & $1.03-3.24$ & 0.115 & 0.025 \\
\hline & & & & & & & & \\
& & & & & &
\end{tabular}

Table III shows the parameters involved in the solution of (7.4) and (16.14) and the quantities

$$
\operatorname{erj}=\sqrt{\frac{\sum_{i}\left(j_{\text {SME }}\left(r_{i}\right)-j_{\exp }\left(r_{i}\right)\right)^{2}}{\sum_{i}\left(j_{\exp }\left(r_{i}\right)\right)^{2}}}, \quad \operatorname{erp}=\sqrt{\frac{\sum_{i}\left(p_{\text {SME }}\left(r_{i}\right)-p_{\exp }\left(r_{i}\right)\right)^{2}}{\sum_{i}\left(p_{\exp }\left(r_{i}\right)\right)^{2}}}
$$

which are a measure of the adherence of the theoretical current density and pressure profiles to the experimental ones in the region $s a \lambda \leq r \leq s a$. The loop voltage $U(V)$ is not critical for the determination of the profiles because a change of $U$ can be compensated to some extent by a change of $\mu$ as is clear by inspecting equation (7.4). The values $\mu s a<1$ indicated in the table are those below which the profiles become insensitive to the value of $\mu s a$. 
Figure 10. The mean square deviation of the theoretical current density profile with respect to the experimental profile when $j_{S M E}$ and $j_{\exp }$ are normalized on inner boundary surfaces displaced by $\Delta q=q-1$ (from Minardi E.; Sozzi C.; Mantica P. Nucl. Fusion 2008, 48, 045001) (with permission).

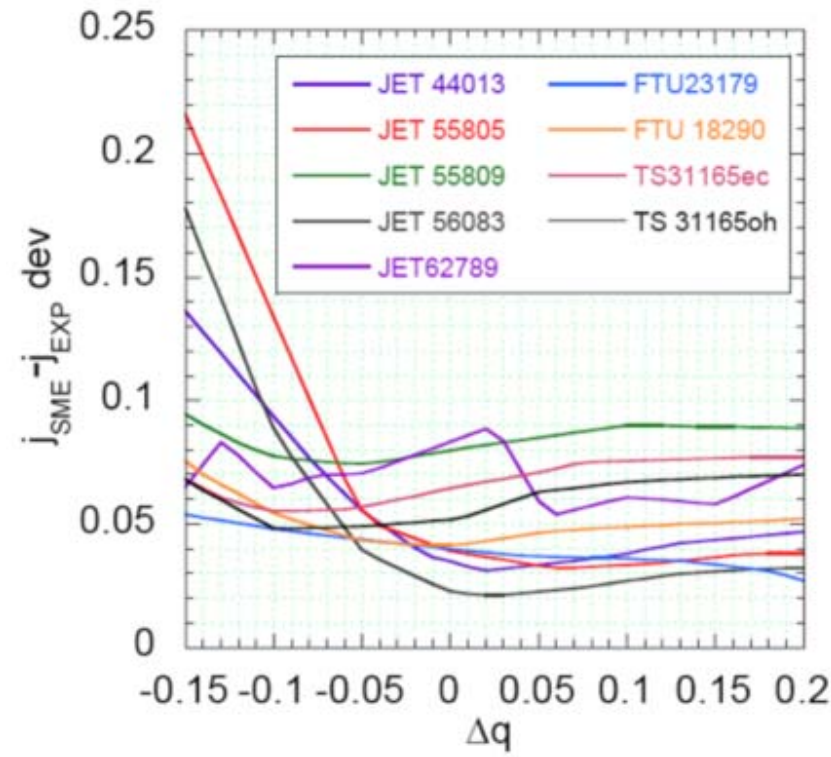

Figure 11. Dependence of the normalized current density on $\Psi=\psi / 2 \pi \mathrm{s}^{2} a^{2}$ (where $\psi$ is the experimental poloidal flux with $\psi(a)=0$, measured in $\mathrm{T}-\mathrm{m}^{2}$ ) in the range from $q=1$ to $r=0.85 \mathrm{~m}$, according to equilibrium reconstruction (a) L-states; (b) H-states (from Minardi E.; Sozzi C.; Mantica P. Nucl. Fusion 2008, 48, 045001) (with permission).
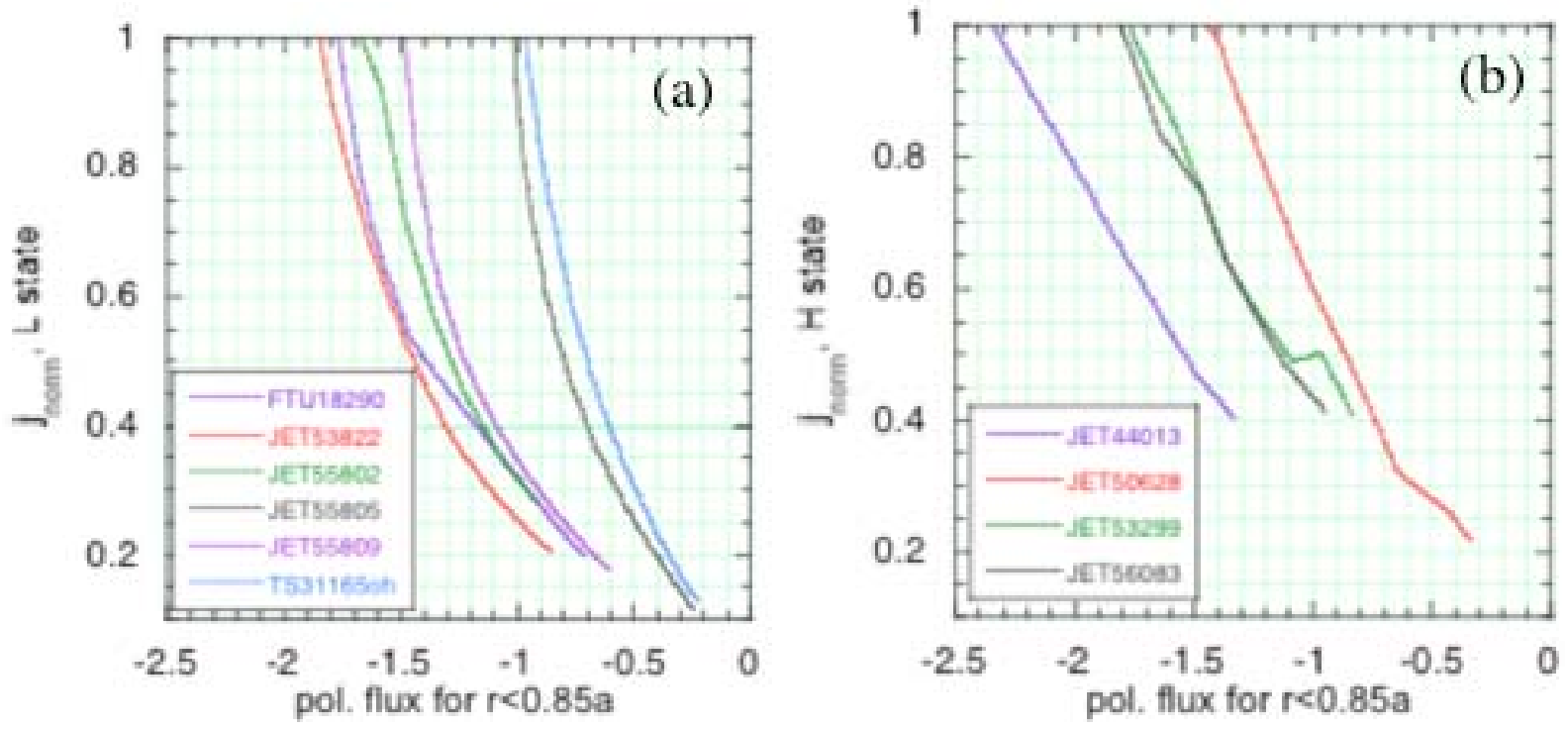

The value of $s$ should correspond to the upper limit of the confinement region. A good fit is generally obtained for $0.75<s<0.95$ with a mean square deviation (21.1) lower (often much lower, see Table III) than $10 \%$. It is worthwhile to note that the values of $s$ listed in the Table are not critical. For instance, taking $s=0.80$ for all shots one obtains $\overline{\operatorname{erj}}=0.061$ and $\overline{\operatorname{erp}}=0.035$ after averaging over 
the shots, to be compared with the averages $\overline{\text { erj }}=0.055$ and $\overline{\operatorname{erp}}=0.037$ of the optimal values listed in Table II. For $s=0.90$, one obtains $\overline{\operatorname{erj}}=0.085$ and $\overline{\operatorname{erp}}=0.037$, which indicates the worsening of the theory when approaching the edge. It is also noted that, in the average, the agreement with the observations is better for the pressure than for the current density.

In the presence of sawteeth the inner boundary $\lambda s a$ should be fixed in the neighborhood of the surface $q=1$. The criticality of $\lambda s a$ is illustrated in fig.10. The mean square deviation is calculated from (21.1) where the theoretical current density is normalized at the experimental value on the inner boundary surface $q=1$ and the experimental current density is normalized to its value at a displaced surface $q=1+\Delta q$. The mean square deviation is plotted as function of the position of this surface, labeled by $\Delta q$, and one sees that it remains significantly below $10 \%$, and practically insensitive to $q$ for $q>1$. At the contrary, in many cases, the deviation increases rapidly when $q$ decreases below one, denoting a worsening of the adherence between the theoretical and the experimental current density profiles in the region dominated by the sawteeth.

It is worthwhile to point out a difference in the dependence of the current density on the poloidal flux in the $\mathrm{L}$ and $\mathrm{H}$ discharges, which fact could be useful for the understanding of the $\mathrm{L}-\mathrm{H}$ transition. As shown by some examples in fig.11, the $j(\psi(r))$ curve (where the entire radial dependence of $j$, reconstructed from the experimental data, is expressed through $\psi(r)$ with $\psi(a)=0)$ is concave in the case of the L shots (fig.11a) and is approximately linear in the case of the $\mathrm{H}$ shots (fig.11b) up to $r \approx 0.85 a$. This can be associated with the fact, known from section 5 (see also eq.(8.4) where $\varphi$ can be neglected), that in an isolated system, the current density tends to be a linear function of $\psi$ (or of the vector potential), provided that the transition is sufficiently rapid for considering the system as isolated from the external world during the transition time. However the observation above needs further confirmations.

Typical situations extracted from the list of Table II were illustrated in detail (see [21]) by plotting the experimental and theoretical current density and normalized pressure profiles, as well as the experimental non-ohmic net power deposition $p_{E}$ on electrons, which is a mark of the discharge.

One should distinguish between shots with $q_{0}<1$ or $q_{0}>1$. One finds invariably that the SME profiles agree with the experimental ones outside the sawtooth region or in a region where the induced current is not strongly modified by external interventions as non-inductive currents and ramps-up in order to obtain $q_{o}>1$ and generate an internal transport barrier (for further evidence on the profile consistency of the pressure and on the effects of transport barriers see refs.[26-27]).

In the cases with $q_{0}>1$ (shots JET 53298, 58148, 59397, 59211, 53506; see tables II and II) the SME equation is solved in the whole interval $0 \leq r \leq s a$ taking $d j / d r=0$ at $r=0$ and the experimental value at $r=s a$. In these cases one finds that the SME profiles disagree in the centre but rejoin the experimental one out of the region where the current density has a hole and non-inductive effects are important. Indeed it is not possible to find discharges that have $q_{0}>1$ (no sawteeth) in stationary conditions without having significant non-inductive current components. In figs.12 and 13 we present two typical cases with $q_{0}<1$ and $q_{0}>1$ extracted from the set of figures discussed in the quoted paper (see [21]). Fig.12 shows the good adherence to the experimental profiles of shot JET 55809 (NBI+ICR heated L state with 0.27MA of non-inductive current) taken at 49s in the region outside the sawteeth. Fig.13 shows the case of an intense non-inductive current in the central region (JET 58148NBI+ICR heated H-state at 47s) and shows how the theoretical profile of the current 
Figure 12. JET55809 NBI+ICR heated L-state; experimental (symbols) and theoretical (line) profiles of the current density (a) and of the pressure (b) normalized on the $q=1$ surface (from Minardi E.; Sozzi C.; Mantica P. Nucl. Fusion 2008, 48, 045001) (with permission).
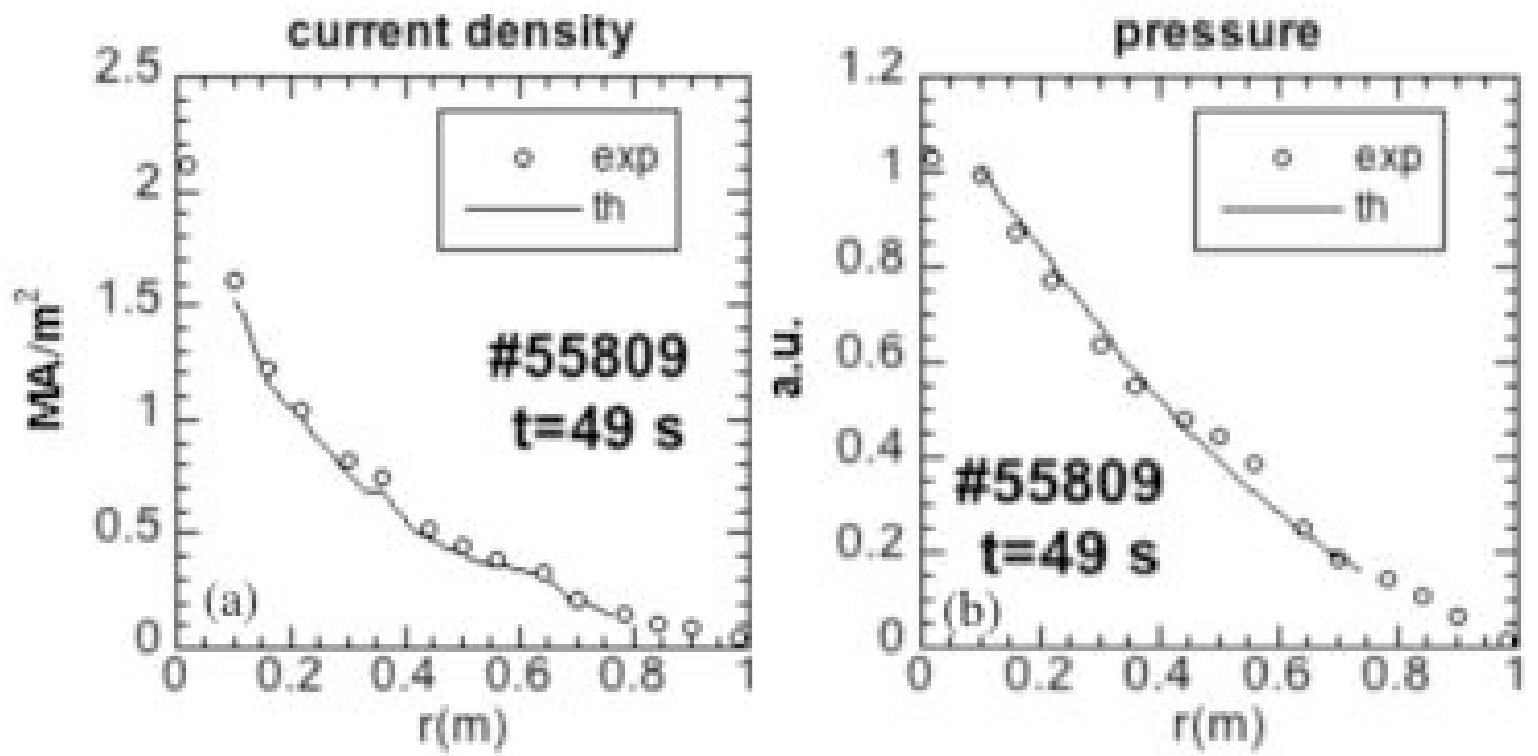

Figure 13. JET58148 NBI+ICR heated H-state with intense non-inductive current in the central region ( $q_{\min } \approx 1.6$ ); (a) total theoretical (line) and experimental (circles) current density and the non-inductive part (full squares); (b) theoretical (line) and experimental (circles) profiles of the current density and of its non-inductive part (full squares) in the outside zone $2.17<q<5.36$ with $r(m)>0.36$; (c) experimental and theoretical normalized pressure in the outside zone (from Minardi E.; Sozzi C.; Mantica P. Nucl. Fusion 2008, 48, 045001) (with permission
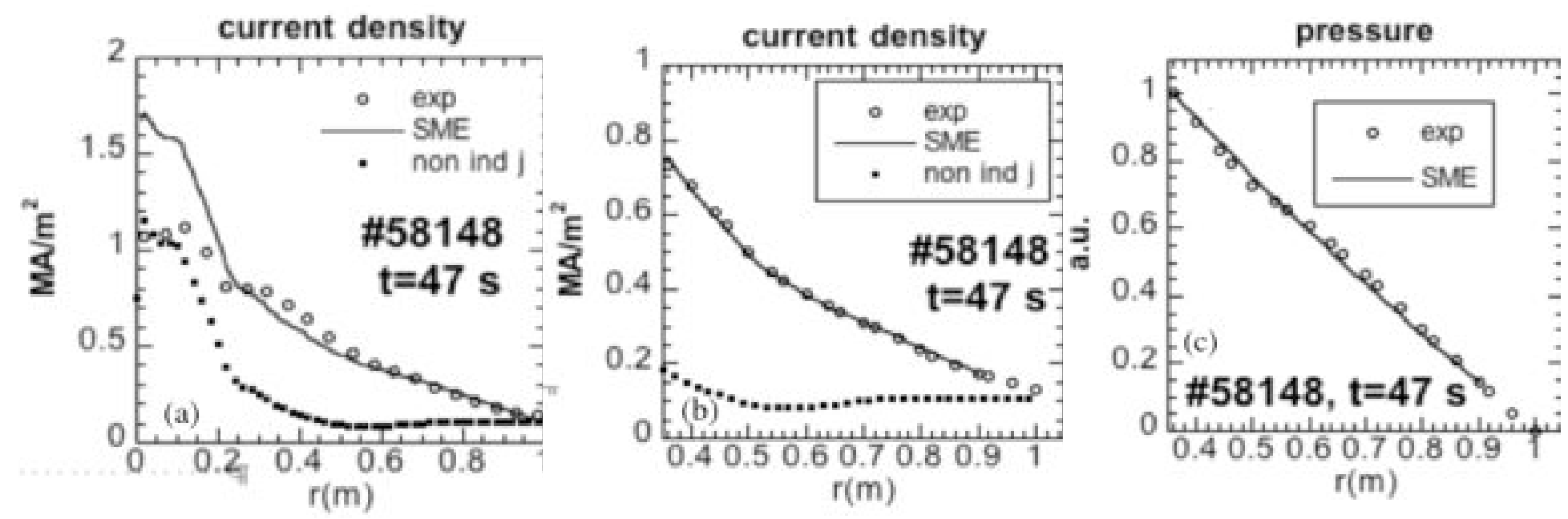

density rejoins the experimental one outside the region of large non-inductive current (fig13(a)). We then solve the SME equations in the outside region i.e. $0.36<r(m)<0.90$, taking the experimental values of $j$ and $p$ at the inner and outer borders. With this additional information the theoretical and 
experimental profiles of the current density and of the pressure agree in the region above, as shown in the figs.13(b) and 13(c). The other shots with $q_{0}>1$ listed in Table II are treated with the same procedure as above: first one solves the SME equation in the whole interval from $r=0$ to $r=s a$ in order to determine the region of adherence between theory and experiment which turns out to be just outside the zone where non inductive currents are important. Then the SME equation is solved again in this region with the appropriate boundary conditions.

Fig. 14 is an example (JET 50630) of a $\mathrm{H}$ discharge with significant non-inductive current $(\approx 0.35 \mathrm{MA})$ in a high density plasma $\left(n_{e 0} \approx 0.71 \times 10^{20} \mathrm{~m}^{-3}\right)$. The non-inductive current density is localized in the central region and around the $q=1$ surface $\left(j_{\text {nind }} \approx 0.15 \mathrm{MA} / \mathrm{m}^{-2}\right)$. The anomalous high value of $\mu s a$ ( $\mu s a \approx 1.70$ ) is due to the effect of the non-inductive current density on the boundary condition at $q=1$ for the inductive part of the current density described by the SME equation. By solving the SME equation in the region $r(m)>0.30, q>1.10$ where the non-inductive current becomes much smaller $\left(j_{\text {nind }} \leq 0,05 \mathrm{MAm}^{-2}\right.$ ), one finds essentially the same values of erj and erp as in table III, independent of $\mu s a$ for $\mu s a<0.50$.

Figure 14. JET50630 NBI+ICR heated H-state with high density $\left(n_{e}(0) \times 10^{-20}=0.70\right)$; theoretical (line) and experimental (symbols) profiles of the total current density (a), including 0.22MA of non-inductive current and of the pressure (b) in the range $1.03<q<2.78$ (from Minardi E.; Sozzi C.; Mantica P. Nucl. Fusion 2008, 48, 045001) (with permission).
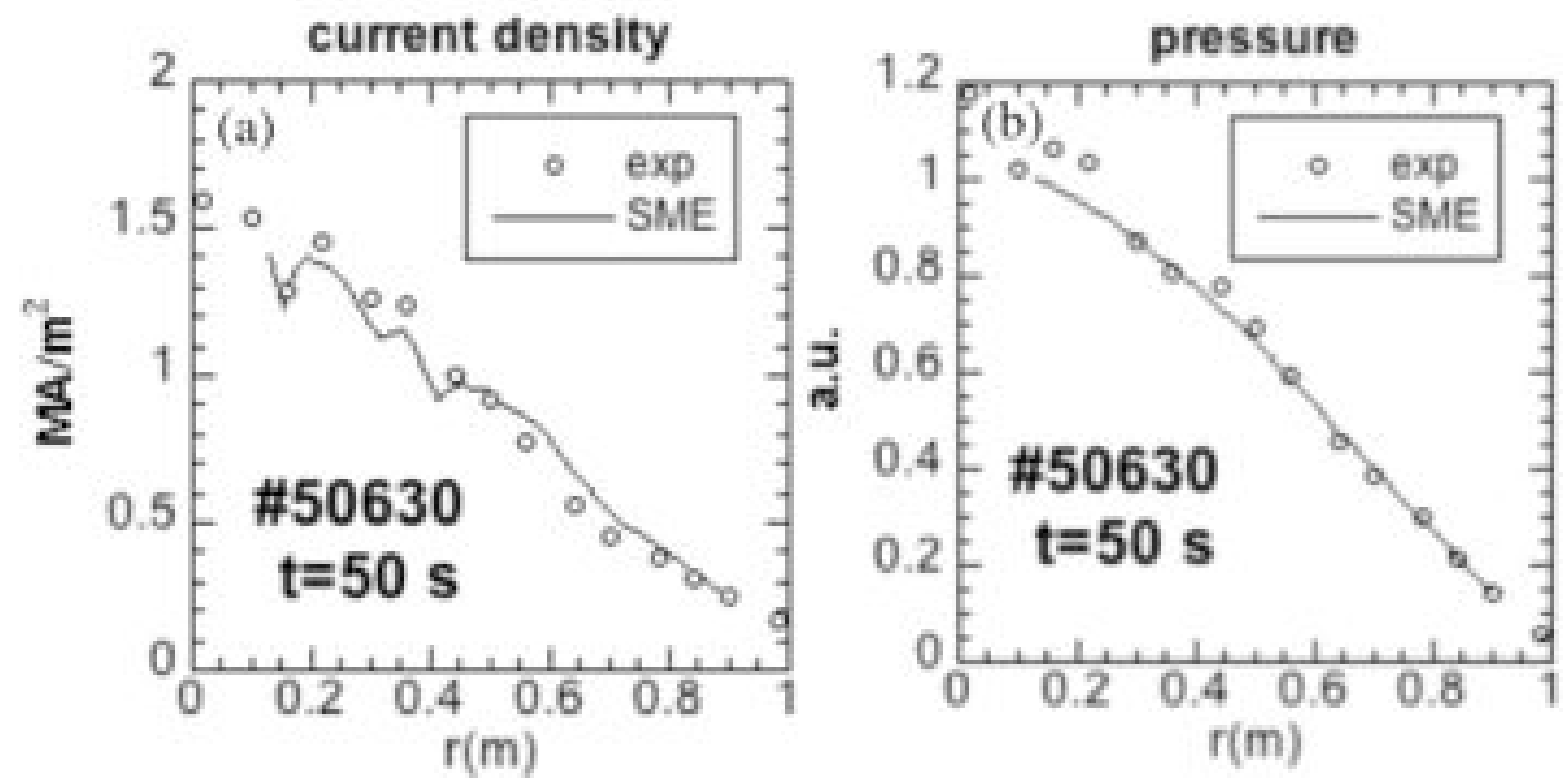

\section{CHAPTER 7: Connexion between magnetic entropy and Lagrangian description of the particle motion}

Summary: In this chapter the relation between the magnetic entropy and the Lagrangian motion of the underlying system of particles is investigated. We consider the motion of independent groups of $N_{j}$ particles, each group being identified by the volume element $\Delta V_{j}$ in which the guiding centers of 
the $N_{j}$ particles were contained at the initial instant of time. Radiation effects are neglected and the magnetic field is treated as a given, albeit "a priori”, unspecified function of space.

The Lagrangian motion is constrained by the condition that the system of particles contributes to an organized (macroscopic) motion such that the total interaction energy of the system of particles with the macroscopic vector potential is fixed. This macroscopic constraint is the counterpart of the constraint (2.3), which is at the base of the introduction of the interaction energy $\Phi_{\text {int }}$ in chapter 1 . This condition, together with the coarse-graining involved by the partition of the total volume into volume elements $\Delta V_{j}$ allows for establishing a correspondence between the Lagrangian description of the motion and the properties of the magnetic entropy.

It is a characteristic feature of the formalism, which results from the macroscopic constraint above, that the Lagrange equations of motion imply simultaneously the equations describing the motion at the single particle level and the equation describing the macroscopic motion under stationary conditions.

The Lagrangian $L$ averaged, at a given instant of time, applying a Maxwellian distribution to the single particle velocities, is related to the magnetic entropy $S_{p}$ by the equality $\bar{L}=U+T S_{p}$ where $U$ is the thermal energy $(3 / 2) \int n T d V$.

It will be shown that the present thermodynamic description holds at scale lengths much larger than the screening length $l_{e}=\left(m c^{2} / 4 \pi n q^{2}\right)^{1 / 2}$ arising in the system of electromagnetically interacting particles [1], [29].

The quantities introduced in chapter 1 , for instance the generalized temperature $\tau$, will find their physical interpretation through the process of average.

The Lagrangian $L$ can be split in a part $\tilde{L}_{p}$ that describes the motion at the single particle level and in a part $L_{X}$, related to the macroscopic configuration. This separation allows for treating the collective part independently of any assumption on the velocity distribution at the single particle level. The collective part is averaged applying the coarse-grained canonical distribution (3.5). It is shown that the vanishing of the averaged variation $\delta \overline{L_{X}}$ with respect to suitably defined variations of the equilibrium implies $\delta S_{p}=0, \delta \Phi_{\text {int }}=0$ and that the vector potential must satisfy the equation $\nabla \times \nabla \times \vec{A}_{p}+\mu^{2} \vec{A}_{p}=0$ in an isolated system and in the limit $\left(l_{e} \mu\right)^{2}<<1$.

When one assumes that, in the same limit, the canonical average of the variation $\delta L$ of $L=L_{X}+\tilde{L}_{p}$ is equal to its time average, one finds an equivalence relation between the Hamilton's action principle and the vanishing of the first variation of the magnetic entropy.

\section{Lagrangian motion of the particles with macroscopic constraints}

We start from the Lagrangian of a system of $N_{p}$ independent particles with mass $m$ and charge $q$ moving with velocity $\vec{V}_{n}=d \vec{x}_{n} / d t$ in a magnetic field described by the vector potential $\overrightarrow{\mathrm{A}}(\vec{x}, t)$ :

$$
L(t)=\frac{m}{2} \sum_{n=1}^{N_{p}} \vec{V}_{n}^{2}+\frac{q}{c} \sum_{n=1}^{N_{p}} \vec{V}_{n} \cdot \vec{A}\left(\vec{x}_{n}, t\right) \text {, }
$$

where

$$
\frac{\partial \vec{A}\left(\vec{x}_{n}, t\right)}{\partial}=-c E\left(\vec{x}_{n}, t\right)
$$


For comparison with the point of view of the magnetic entropy concept, we must see how the coarse-graining of the magnetic configuration and the constraint (2.3) implied basically by this concept, can be taken into account in the Lagrangian formulation of the motion.

We consider the Lagrangian at a given instant of time $t_{0}$ and divide the particles into groups whose guiding centers are contained at this time into volume elements $\Delta V$.

The average velocity of the particles present with their guiding centers in the element $\Delta V_{j}$ at $t_{o}$ is then given by the expression

$\vec{u}_{j}\left(t_{0}\right)=\frac{1}{N_{j}} \sum_{n=1}^{N_{j}} \vec{V}_{n}\left(t_{0}\right)=\frac{1}{N_{j}} \sum_{n=1}^{N_{j}} \frac{d \vec{x}_{n}}{d t}$,

where $N_{j}>>1$ is the number of such particles at that instant.

We now look at the equation of motion of the group of $N_{j}$ particles, thus identified at $t=t_{0}$, as is described for $t>t_{0}$ by the action principle involving the Lagrangian (22.1). The variation of the action will be calculated under the condition that the motion is associated with a prescribed value of the quantity

$\left.\frac{q}{2 c} \sum_{j=1}^{N} n_{j} \vec{u}_{j} \cdot \vec{A}\left(\vec{X}_{j}, t\right)\right) V_{j}$

where $n_{j}=N_{j} / \Delta V_{j}$ is the density and

$\vec{X}_{j}(t)=\left(1 / N_{j}\right) \sum_{n=1}^{N_{j}} \vec{x}_{n}(t), \quad \vec{u}_{j}(t)=d \vec{X}_{j} / d t=\frac{1}{N_{j}} \sum_{n=1}^{N_{j}} \vec{V}_{n}(t)$.

The constraint (22.4) expresses the fact that the magnetic interaction energy of the system of particles with the macroscopic part of the vector potential is fixed. The value of (22.4) will be determined in the next section where (22.4) will be compared with the similar constraint (2.3) introduced in section 2 for the derivation of the magnetic entropy.

Introducing the Lagrangian multiplier $\lambda$, the Lagrangian of the constrained motion is expressed as $L(t)=\frac{m}{2} \sum_{n=1}^{N_{p}} \vec{V}_{n}^{2}+\frac{q}{c} \sum_{n=1}^{N_{p}} \vec{V}_{n} \cdot \vec{A}\left(\vec{X}_{n}, t\right)+\lambda \frac{q}{2 c} \sum_{j=1}^{N} \sum_{n=1}^{N_{j}} \vec{V}_{n} \cdot \vec{A}\left(\vec{X}_{j}, t\right)$.

Let us single out the $k$-td particle belonging to the j-td group of $N_{j}$ particles. The equation of motion is given by

$\frac{d}{d t} \nabla_{\vec{V}_{k}} L-\nabla_{\vec{x}_{k}} L=0$.

We have

$\frac{d}{d t} \nabla_{\vec{V}_{k}} \sum_{j}^{N} \sum_{n=1}^{N_{j}} \vec{V}_{n} \cdot \vec{A}\left(X_{j}, t\right)=\frac{d}{d t} \vec{A}\left(\vec{X}_{j}, t\right)=\sum_{n=1}^{N_{j}} \vec{V}_{n} \cdot \nabla_{\vec{X}_{n}} \vec{A}\left(\vec{X}_{j}, t\right)+\frac{\partial \vec{A}\left(\vec{X}_{j}, t\right)}{\partial}=$

$=\frac{1}{N_{j}} \sum_{n=1}^{N_{j}} \vec{V}_{n} \cdot \nabla_{\vec{X}_{j}} \vec{A}\left(\vec{X}_{j}, t\right)+\frac{\partial \vec{A}\left(\vec{X}_{j}, t\right)}{\partial}=\vec{u}_{j} \cdot \nabla_{\vec{X}_{j}} \vec{A}\left(\vec{X}_{j}, t\right)+\frac{\partial \vec{A}\left(\vec{X}_{j}, t\right)}{\partial}$.

(Note that $\left.\nabla_{\vec{x}_{n}} \vec{X}_{j}=\left(1 / N_{j}\right) \nabla_{\vec{X}_{j}}\right)$. The first transition in (22.8) is a consequence of the fact that only the $k$-td particle among the $N_{j}$ particles contributes to the derivation; the subsequent equalities follow from the relations (22.5). The contribution of the $\lambda$-term to the equation of motion is then $\frac{\lambda q}{2 c}\left(\frac{d}{d t} \nabla_{\vec{V}_{k}}-\nabla_{\vec{x}_{k}}\right) \sum_{j=1}^{N} \sum_{n=1}^{N_{j}}\left(\vec{V}_{n} \cdot \vec{A}\left(\vec{X}_{j}, t\right)\right)=$ 


$$
\begin{aligned}
& =\frac{\lambda q}{2 c}\left(\frac{\partial \vec{A}\left(\vec{X}_{j}, t\right)}{\partial}+\vec{u}_{j}(t) \cdot \nabla_{\vec{X}_{j}} \vec{A}\left(\vec{X}_{j}, t\right)-\nabla_{\vec{X}_{j}} \vec{u}_{j}(t) \cdot \vec{A}\left(\vec{X}_{j}, t\right)\right)= \\
& =-\frac{\lambda q}{2 c}\left(c \vec{E}\left(\vec{X}_{j}, t\right)+\vec{u}_{j}(t) \times \vec{B}\left(\vec{X}_{j}, t\right)\right),
\end{aligned}
$$

and the equation of motion takes on the form

$$
m \frac{d \vec{V}_{k}(t)}{d t}=q\left(\frac{1}{c} \vec{V}_{k} \times \vec{B}\left(\vec{x}_{k}, t\right)+\vec{E}_{e x t}\left(\vec{x}_{n}, t\right)\right)+\lambda \frac{q}{2}\left(\frac{1}{c} \vec{u}_{j} \times \vec{B}\left(\vec{X}_{j}, t\right)+\vec{E}\left(\vec{X}_{j}, t\right)\right) .
$$

Let us sum both members of (22.10) over the group of $N_{j}$ particles at the time $t=t_{0}$ when all $N_{j}$ particles are in $\Delta V_{j}$. After dividing by $N_{j}$ one has

$$
m \frac{1}{N_{j}}\left(\sum_{k=1}^{N_{j}} \frac{d \vec{V}_{k}}{d t}\right)_{t=t_{0}}=\left(q+\lambda \frac{q}{2}\right)\left(\frac{1}{c} \vec{u}_{j} \times \vec{B}\left(\vec{X}_{j}, t_{0}\right)+\vec{E}\left(\vec{X}_{j}, t_{0}\right)\right)
$$

where we have supposed that $\vec{B}$ is practically uniform in $\Delta V_{j}$ so that $\vec{B}\left(\vec{X}_{k}, t_{0}\right)=B\left(\vec{X}_{j}, t_{0}\right)$. We shall see in the next section that $\lambda$ is very close to -2 but it cannot be identical to it. This means that $\left(d \vec{u}_{k} / d t\right)_{t=t_{0}}=\left(1 / N_{j}\right)\left(\sum_{k=1}^{N_{j}} d \vec{V}_{k} / d t\right)_{t=t_{0}}$ is very close to 0 and that the macroscopic system tends to be stationary, even though not exactly stationary. If we want to eliminate any arbitrariness or indefinites in our conclusions about the behavior of the system, while remaining consistent with the result above, we are forced to admit that the two members of eq.(22.11) must vanish independently. So we obtain the equations

$$
\left(\frac{d \vec{u}_{j}(t)}{d t}\right)_{t=t_{0}}=0, \quad \frac{1}{c} \vec{u}_{j} \times \vec{B}\left(\vec{X}_{j}, t_{0}\right)+\vec{E}\left(\vec{X}_{j}, t_{0}\right)=0 .
$$

Since this reasoning can be repeated for any group of $N_{j}$ particles in any $\Delta V_{j}$ and at any time $t=t_{0}$, we conclude that, under our assumptions (in particular eq.(22.4)), the motion at the collective level should be always stationary everywhere and described by the equations (22.12) at any time.

Taking into account (22.12), eq. (22.10) gives

$$
m \frac{d \vec{V}_{k}(t)}{d t}=q\left(\frac{1}{c} \vec{V}_{k} \times \vec{B}\left(\vec{x}_{k}, t\right)+\vec{E}\left(\vec{x}_{k}, t\right)\right) \text {. }
$$

This equation describes the motion at the level of the individual particles. Thus the introduction of the constraint (22.4) has made possible a unified Lagrangian description of the motion at the two levels. The macroscopic equation of motion can be completed with the introduction of the effects of the pressure tensor $\vec{P}$ and of the dissipative forces $\vec{R}$. This can be done in a phenomenological way, according to a standard procedure of classical mechanics [28], by defining

$$
\delta \int_{t_{1}}^{t_{2}} L(t) d t=\sum_{n} \int_{t_{1}}^{t_{2}}\left(\frac{\partial}{\partial \vec{x}_{n}}-\frac{d}{d t} \frac{\partial L}{\partial \vec{V}_{n}}\right) \cdot \delta \vec{x}_{n} d t=\left(1+\frac{\lambda}{2}\right) \sum_{j=1}^{N} \sum_{n=1}^{N_{j}} \int_{t_{1}}^{t_{2}} \vec{F}\left(\vec{X}_{j}(t)\right) \cdot \delta \vec{x}_{n} d t,
$$

where

$$
\vec{F}\left(\vec{X}_{j}\right)=\frac{1}{n}(\nabla \cdot \vec{P}-\vec{R})
$$

By following the same lines as above we arrive at an equation of the form (22.11) involving the term $F\left(\vec{X}_{j}\right)$ so that the macroscopic equation of motion becomes 


$$
\frac{q}{c}\left(\vec{u}_{j} \times \vec{B}\left(\vec{X}_{j}, t\right)+\vec{E}\left(\vec{X}_{j}, t\right)\right)-\frac{1}{n}(\nabla \cdot \vec{P}-\vec{R})=0
$$

\section{The relation between the magnetic entropy and the Maxwellian average of the Lagrangian}

In this paragraph we assume that the velocity distribution of the particles are Maxwellians with local temperature in each $\Delta V_{j}$ and centered at an average velocity consistent with the macroscopic description of our model. We wish to see what form the Lagrangian (22.6) will take when the single particle velocities are averaged, at a given instant $t_{0}$, with the Maxwellian distribution. This will enable us to see a relation between the Lagrangian description of the motion of the system of particles and the description of the macroscopic system based on the magnetic entropy and to gain a better physical insight of the latter. For this purpose we shall express $S=S_{p}+S_{b}$ in terms of the velocities rather than in terms of the current density.

To simplify the discussion, we shall take the density $n(\vec{X})$ as uniform and fixed. That is, we assume that the number of particles $N_{j}$ at $t=t_{0}$ is fixed and equal for all $\Delta V_{j}$. In fact the fluctuations of $\vec{J}_{j}=q N_{j} \vec{u}_{j} / \Delta V_{j}$ ( $q$ is the electron charge) arising from the Poisson's fluctuations of $N_{j}$ in $\Delta V$ (with $\left.N_{j}>>1\right)$ are negligible with respect to the fluctuations arising from the random velocity $\overrightarrow{\widetilde{v}}_{n}$.

We suppose that an organized or macroscopic velocity distribution $\vec{v}(\vec{X})$ is present in the system superimposed on a random velocity $\overrightarrow{\widetilde{v}}_{n}$. That is to say, the particle velocity $\vec{V}_{n}$ measured at any given time $t_{0}$ is composed of a random part $\overrightarrow{\widetilde{v}}_{n}$ and of a collective part $\vec{v}\left(\vec{X}_{j}\right)$, considered as a given function of $\vec{X}_{j}$ and supposed as essentially uniform in each $\Delta V_{j}$ so that $\vec{v}\left(\vec{x}_{n}\right)=\vec{v}\left(\vec{X}_{j}\right)$ for any $\vec{x}_{n} \in \Delta V_{j}$.

Thus,

$\vec{V}_{n}=\vec{v}\left(\vec{X}_{j}\right)+\overrightarrow{\widetilde{v}}_{n}$ for $\vec{x}_{n}\left(t_{0}\right), \vec{X}_{j}\left(t_{0}\right) \in \Delta V_{j}$.

The average velocity (22.3) is then

$\vec{u}_{j}=\vec{v}\left(\vec{X}_{j}\right)+\overrightarrow{\widetilde{V}}_{j}$,

where $\overrightarrow{\widetilde{V}}_{j}=\frac{1}{N_{j}} \sum_{n=1}^{N_{j}} \overrightarrow{\widetilde{v}}_{n} \quad$ for $\vec{X}_{n}\left(t_{0}\right) \in \Delta V_{j}$.

Correspondingly the current density is divided in a collective part $\vec{j}\left(X_{j}\right)$ and in a fluctuating part $\overrightarrow{\tilde{j}}_{j}$ according to the expression

$\vec{J}_{j}=q n \vec{u}_{j}=q n \vec{v}\left(\vec{X}_{j}\right)+n q \overrightarrow{\widetilde{V}}_{j}$

Here $\vec{J}_{j}$ and $\vec{j}\left(\vec{X}_{j}\right)$ are the same quantities introduced in chapter 1 ; in particular $\overline{\tilde{j}^{2}}=n^{2} q^{2} \overline{\widetilde{V}^{2}}$ satisfies the relation (4.9).

In accordance with the constraint (2.3) of the statistical model, the velocity $\overrightarrow{\widetilde{V}}_{j}$ is not vanishing in general but it results from the effect of the collective field on the fluctuating background described by the interaction energy $\Phi_{\text {int }}$. In absence of this interaction the collective system and the background are isolated and the average $\overline{\vec{V}}_{j}$ vanishes (see (17.3) and (3.7b), where $\overline{\vec{j}}_{j}=n q \overline{\overrightarrow{\widetilde{V}}}_{j}$ ). 
We consider a plasma magnetic state in the absence of electric field, so that the collective vector potential $\vec{A}_{p}\left(\vec{X}_{j}\right)$ does not depend explicitly on time. Then, taking into account (23.2), the condition (22.4) becomes (see (2.5) and (2.6))

$$
\frac{q}{2 c} \sum_{j=1}^{N} n_{j}\left(\vec{v}\left(\vec{X}_{j}\right)+\vec{V}_{j}\right) \cdot \vec{A}_{p}\left(\vec{X}_{j}\right) \Delta V_{j}=\Phi+\widetilde{\Phi}_{\mathrm{int}}
$$

where

$\frac{q}{2 c} \sum_{j=1}^{N} n_{j} \overrightarrow{\widetilde{V}}_{j} \cdot A_{p}\left(\vec{X}_{j}\right) \Delta V_{j}=\widetilde{\Phi}_{\mathrm{int}} \cdot$

When the organized velocity $\vec{v}\left(\vec{X}_{j}\right)$ is subtracted from the velocity (23.2) averaged in $\Delta V_{j}$, still remains the average contribution $\overline{\vec{V}}_{j}$ of the fluctuations $\overrightarrow{\widetilde{v}}_{n}$ in $\Delta V_{j}$, due to the non-vanishing interaction $\Phi_{\text {int }}$. Consistently with (3.7a), this average velocity for the group of $N_{j}$ particles in $\Delta V_{j}$ at $t=t_{0}$ is given by $\overline{\overrightarrow{\widetilde{V}}_{j}}=-\vec{v}\left(\vec{X}_{j}\right)+\left(\mu^{2} c / 4 \pi n q\right) \vec{A}_{p}\left(\vec{X}_{j}\right)$. We then consider that the distribution of the $\vec{v}_{n}$ velocities of the $N_{j}$ particles in $\Delta V_{j}$ is a Maxwellian centered in $\overline{\vec{V}}_{j}$ and with temperature $T_{j}$, namely we put

$$
f\left(\overrightarrow{\widetilde{V}}_{1} \ldots \overrightarrow{\widetilde{v}}_{N_{j}}\right)=\prod_{n=1}^{N_{j}} f_{n, j}\left(\overrightarrow{\widetilde{v}}_{n}-\overrightarrow{\vec{V}}_{j}\right)
$$

where

$$
f_{n, j}\left(\overrightarrow{\widetilde{v}}_{n}-\overline{\vec{V}}_{j}\right)=\left(\frac{m}{2 \pi T_{j}}\right)^{3 / 2} \exp -\frac{m\left(\overrightarrow{\widetilde{v}}_{n}-\overline{\vec{V}}_{j}\right)^{2}}{2 T_{j}} .
$$

By averaging (23.3) with the distribution (23.6) one obtains

$$
\overline{\vec{V}}_{j}=\frac{1}{N_{j}} \sum_{n=1}^{N_{j}} \int \overrightarrow{\widetilde{v}}_{n} f d^{3} \tilde{v}_{1} \ldots d^{3} \widetilde{v}_{N_{j}}=-\vec{v}\left(\vec{X}_{j}\right)+\left(\frac{\mu^{2} c}{4 \pi}\right) \vec{A}_{p}\left(\vec{X}_{j}\right) \text {. }
$$

Then the average of (23.5) is $\overline{\widetilde{\Phi}}_{\mathrm{int}}=\Phi_{\mathrm{int}}$ where $\Phi_{\mathrm{int}}$ is the same as (3.7b) or (5.5).

Using (23.1), the Lagrangian (22.6) at the time $t_{0}$ takes on the form

$$
\begin{aligned}
& L\left(t_{0}\right)=\frac{m}{2} \sum_{j=1}^{N} \sum_{n=1}^{N_{j}} \widetilde{v}_{n}^{2}+\frac{m}{2} \sum_{j=1}^{N}\left(\vec{v}^{2}\left(\vec{X}_{j}\right)+2 \vec{v}\left(\vec{X}_{j}\right) \cdot \overrightarrow{\widetilde{V}}_{j}\right) n \Delta V_{j}+ \\
& +\frac{q}{c} \sum_{n=1}^{N_{p}} \vec{V}_{n} \cdot \vec{A}_{p}\left(\vec{x}_{n}\right)+\frac{\lambda}{2} \frac{q}{c} \sum_{j=1}^{N} n_{j} \vec{u}_{j} \cdot \vec{A}_{p}\left(\vec{X}_{j}\right) \Delta V_{j} .
\end{aligned}
$$

The first term can be expressed with the Maxwellian temperature after performing the average with a Maxwellian distribution of the single particle velocities $\overrightarrow{\widetilde{v}}_{n}$. One obtains $\left(1 / N_{j}\right) \sum_{n=1}^{N_{j}}{\overline{\overrightarrow{v_{n}}}}^{2}=3 T_{j} / m$ (we assume $\left.|\bar{V}|<<(2 T / m)^{1 / 2}\right)$, so that the term becomes equal to the plasma thermal energy

$$
\frac{m}{2} \sum_{j=1}^{N} \sum_{n=1}^{N_{j}}{\overline{\overrightarrow{v_{n}}}}^{2}=\frac{3}{2} \sum_{j=1}^{N} N_{j} T_{j}=\frac{3}{2} \sum_{j=1}^{N} n T_{j} \Delta V_{j} \equiv U .
$$

Let us calculate the average of the third term applying the distribution (23.6): 


$$
\begin{aligned}
& m n \int f \sum_{j=1}^{N} \vec{v}\left(\vec{X}_{j}\right) \cdot \overrightarrow{\widetilde{V}}_{j} \Delta V_{j} d^{3} \overrightarrow{\widetilde{v}}_{1} \ldots d^{3} \overrightarrow{\widetilde{v}}_{N_{j}} \\
& =\frac{2 m n \Delta V}{3} \overline{\widetilde{V}}^{2} S_{p}=m n \sum_{j=1}^{N}\left(-\vec{v}^{2}\left(\vec{X}_{j}\right)+\frac{\mu^{2} C}{4 \pi n q} \vec{v}\left(\vec{X}_{j}\right) \cdot \vec{A}_{p}\left(\vec{X}_{j}\right)\right),
\end{aligned}
$$

where the relations (4.3) and (5.1) have been used.

We now consider the fourth term

$\sum_{n=1}^{N_{p}} \vec{V}_{n} \cdot \vec{A}_{p}\left(\vec{x}_{n}\right)=\sum_{j=1}^{N} \sum_{n=1}^{N_{j}} \vec{V}_{n} \cdot \vec{A}_{p}\left(\vec{x}_{n}\right)=\sum_{j=1}^{N}\left(N_{j} \vec{v}\left(\vec{X}_{j}\right) \cdot \vec{A}_{p}\left(\vec{X}_{j}\right)+\sum_{n=1}^{N_{j}} \overrightarrow{\widetilde{V}}_{n} \cdot \vec{A}_{p}\left(\vec{X}_{j}\right)\right)$.

Here we take into account the fact that the positions $\vec{x}_{n}$ of the particles grouped in $\Delta V_{j}$ at the time $t_{0}$ cannot be distinguished due to the coarse-graining of the configuration at the scale of $\Delta V$. Thus $\vec{A}_{p}\left(\vec{x}_{n}\right)$ can be identified with $\vec{A}_{p}\left(\vec{X}_{j}\right)$ for any $\vec{X}_{n} \in \Delta V_{j}$. With this in mind and recalling (23.2) and (23.3) one has

$\sum_{n=1}^{N_{p}} \vec{V}_{n} \cdot \vec{A}_{p}\left(\vec{x}_{n}\right)=\sum_{j=1}^{N} n \vec{u}_{j} \cdot \vec{A}_{p}\left(\vec{X}_{j}\right) \Delta V_{j}$.

This term combines with the last term of (23.9) to give $(\lambda+2)\left(\Phi+\Phi_{\text {int }}\right)$ after taking into account the Maxwellian average of (23.4) where $\vec{u}_{j}$ is defined by (23.2). We are now in position to write the average of (23.9):

$$
\begin{gathered}
\bar{L}=U+\frac{m n}{2} \sum_{j=1}^{N}\left(-\vec{v}^{2}\left(\vec{X}_{j}\right)+\frac{\mu^{2} c}{4 \pi q n} \vec{v}\left(\vec{X}_{j}\right) \cdot \vec{A}_{p}\left(\vec{X}_{j}\right)\right) \Delta V_{j}+(\lambda+2)\left(\Phi+\Phi_{\mathrm{int}}\right)= \\
=U+\frac{m n}{2} \sum_{j=1}^{N}\left(-\vec{v}^{2}\left(\vec{X}_{j}\right)+\left(\frac{\mu^{2} c}{4 \pi q n}+\left(1+\frac{\lambda}{2}\right) \frac{q}{m c}\right) \vec{v}\left(\vec{X}_{j}\right) \cdot \vec{A}_{p}\left(\vec{X}_{j}\right)\right) \Delta V_{j}+(\lambda+2) \Phi_{\mathrm{int}} .
\end{gathered}
$$

The relation above contains the Lagrange multiplier $\lambda$, which should be determined by the constraint (22.4) after prescribing $\Phi+\Phi_{\text {int }}$. However, as is customary in statistical mechanics, one can reverse the procedure and consider instead the Lagrange multiplier $\lambda$ as the primary quantity. This is the way followed in ordinary statistical mechanics for introducing the temperature and indeed we shall see presently that even in the present case $\lambda$ is related to the generalized temperature $\tau$.

We start by observing that if $\lambda$ is chosen in such a way as to satisfy the relation

$\frac{q}{m c}\left(\frac{\mu^{2} c^{2} m}{2 \pi n q^{2}}+\lambda+2\right)=\frac{\mu^{2} c}{4 \pi n q}$ or $\lambda+2=-\frac{\mu^{2} c^{2} m}{4 \pi n q^{2}}$,

the average of the Lagrangian can be expressed as a linear function of the magnetic entropy and of the plasma thermal energy only. Indeed, applying (23.11), $\bar{L}$ takes on the form

$\bar{L}=U+\frac{m n \Delta V}{3}{\overline{\widetilde{V}^{2}}} S_{p}+(\lambda+2) \Phi_{\mathrm{int}}$.

Here $\overline{\widetilde{V}^{2}}$ is defined by (4.2) as $\overline{\widetilde{V}^{2}}=(1 / N) \sum_{j=1}^{N} \overline{\tilde{V}_{j}^{2}}$ where, recalling (23.3),

$\tilde{V}_{j}^{2}=\frac{1}{N_{j}^{2}}\left(\sum_{n=1}^{N_{j}} \widetilde{v}_{n}^{2}+\sum_{n \neq n^{\prime}}^{N_{j}} \sum_{n^{\prime}=1}^{N_{j}} \overrightarrow{\widetilde{v}}_{n} \cdot \overrightarrow{\widetilde{v}}_{n^{\prime}}\right)$.

Since $\overline{\mid \widetilde{V}} \mid<<(2 T / m)^{1 / 2}$, the particle-particle correlation can be neglected, so one has 
$\overline{\widetilde{V}^{2}}=\frac{1}{N} \sum_{j=1}^{N} \overline{\widetilde{V}_{j}^{2}}=\frac{1}{N} \sum_{j=1}^{N} \frac{1}{N_{j}^{2}} \sum_{n=1}^{N_{j}} \overline{\tilde{V}_{n}^{2}}=\frac{3}{m N_{j}} T$,

where $N_{j}=n \Delta V$ and $T=\sum_{j=1}^{N} T_{j} / N$. Thus, the generalized temperature $\tau$ is related, in accordance with

(5.3), to the Maxwellian temperature $T$ by the relation

$\tau=-\frac{4 \pi \Delta V n^{2} q^{2}{\tilde{V^{2}}}^{2}}{3 \mu^{2} c^{2}}=-\frac{4 \pi n q^{2}}{\mu^{2} c^{2} m} T$.

It follows that

$\lambda+2=-\frac{\mu^{2} c^{2} m}{4 \pi n q^{2}}=\frac{T}{\tau}$

and the expression (23.15) for $\bar{L}$ becomes simply

$\bar{L}=U+T\left(S_{p}+S_{b}\right)=U+T S$.

In the case of the tokamak the value of $\lambda$ is arbitrarily close but not identical to -2 . Indeed, as we know (section 17), the value of $\mu^{2}$ is arbitrarily close but not identical to 0 (as would be required by exact minimization of the poloidal magnetic energy within the family of the SME solutions, see section 17). In other cases (the cases considered in section 10 and in chapter 3) $|\mu|^{-1}$ is a characteristic length of the magnetic equilibrium. Taking, say $\mu<(20 \mathrm{~cm})^{-1}, n>10^{13} \mathrm{~cm}^{-3}$ and assuming that the current is sustained by electrons, so that $q$ and $m$ are the electron charge and mass, one finds

$\frac{\mu^{2} c^{2} m}{4 \pi n q^{2}}<10^{-4}$.

The value of $\lambda$ is then very close to -2 ; the parameter $\tau$, which is negative, becomes infinitely negative in the limit $\lambda \rightarrow-2$ and the eq. (23.21) reduces to

$\bar{L}=U+T S_{p}$

The inequality (23.22) has an interesting physical meaning. It means that $\left(\mu l_{e}\right)^{2}<<1$, where $l_{e}=\left(m c^{2} / 4 \pi n q^{2}\right)^{1 / 2}$ is the screening length of the vector potential created by a particle in a system of electromagnetically interacting particles as was discussed first by Bohm and Pines [1]. For a more recent treatment see Essén and Nordmark [29] and references therein (in the theory of the superconductors the length $l_{e}$, with the appropriate definition of $m$ and $q$, describes the penetration of the magnetic field in the superconductor and is called the London penetration length). It follows that the characteristic length of the magnetic plasma equilibria described by the present approach must be much larger than the length below which the effects due to particle discreteness are important and where the particles can be treated as effectively free and the collective effects can be neglected.

The change of entropy of the background $\delta S_{b}=\delta \Phi_{\text {int }} / \tau$ vanishes in the limit $\lambda \rightarrow-2, \tau \rightarrow-\infty$, but is otherwise positive (albeit infinitesimal) when $\delta \Phi_{\text {int }}<0$, for instance when energy is reversibly supplied from the background to the collective system. The parameter $\tau$ can then be interpreted formally as the temperature of the background consistently with the well-known fact that statistical systems with negative temperature are "hotter" than systems with positive temperature, so that their entropy increases when heat is released. 
24. The relation of the first variation of the magnetic entropy to the first variation of the canonically averaged Lagrangian and to the action principle

The relation (23.23) suggests that the variation $\delta S_{p}$ at constant Maxwellian temperature $T$ could be related to the variation $\overline{\delta L}$ of the Maxwellian average of the Lagrangian. In this section we shall investigate the form of this relation when no assumption is made on the probability distribution of the single particle velocities $\vec{v}_{n}$. We shall see that there is a significant implication between this relation and the dynamics of the particles.

We start from the Lagrangian (23.9) taken at a fixed instant of time $t=t_{0}$. This Lagrangian can be split in two parts $L\left(t_{0}\right)=\tilde{L}_{p}\left(t_{0}\right)+L_{X}\left(t_{0}\right)$ where, recalling (23.1), (23.2) and (23.3)

$$
\begin{aligned}
& \widetilde{L}_{p}\left(t_{0}\right)=\frac{m}{2} \sum_{j=1}^{N} \sum_{n=1}^{N_{j}} \widetilde{v}_{n}^{2}+\frac{q}{c} \sum_{j=1}^{N} \sum_{n=1}^{N_{j}} \overrightarrow{\widetilde{v}}_{n} \cdot \vec{A}_{p}\left(\vec{x}_{n}\right)=\frac{m}{2} \sum_{j=1}^{N} \sum_{n=1}^{N_{j}} \widetilde{v}_{n}^{2}+\frac{q}{c} \sum_{j=1}^{N} n \Delta V_{j} \vec{V}_{j} \cdot \vec{A}\left(\vec{X}_{j}\right)= \\
& \frac{m}{2} \sum_{j=1}^{N} \sum_{n=1}^{N_{j}} \tilde{v}_{n}^{2}+2 \widetilde{\Phi}_{\mathrm{int}}, \\
& L_{X}\left(t_{0}\right)=\frac{m}{2} \sum_{j=1}^{N}\left(\vec{v}^{2}\left(\vec{X}_{j}\right)+2 \vec{v}\left(\vec{X}_{j}\right) \cdot \vec{V}_{j}\right) n \Delta V_{j}+\frac{q}{c} \sum_{j=1}^{N} \vec{v}\left(\vec{X}_{j}\right) \cdot \vec{A}_{p}\left(X_{j}\right) n \Delta V_{j} \\
& +\frac{\lambda}{2} \frac{q}{c} \sum_{j=1}^{N} \vec{u}_{j} \cdot \vec{A}_{p}\left(\vec{X}_{j}\right) n \Delta V_{j}
\end{aligned}
$$

The Lagrangian $\tilde{L}_{p}(t)$ describes the unorganized motion at the single particle level and should be ascribed to the background, according to our scheme. The part $L_{X}$ arises from the collective motion of the macroscopic system, that is the motion seen at the coarse-grained level measured by the $\Delta V_{j}$.

We take the average of $L_{X}$ at the instant $t=t_{0}$, applying the canonical distribution (3.5), which in terms of the velocity reads

$$
P=\left(\frac{\alpha}{\pi}\right)^{3 N / 2} \frac{1}{\Delta V^{N}} \exp \left(-\alpha\left(\overrightarrow{\widetilde{V}}_{j}-\overline{\vec{V}}_{j}\right)^{2}\right),
$$

where $\alpha=3 / 2 \overline{\widetilde{V}^{2}}$. One has

$$
\begin{aligned}
& \overline{\vec{V}}_{j}=-\vec{v}\left(\vec{X}_{j}\right)+\left(c \mu^{2} / 4 \pi n q\right) \vec{A}_{p}\left(\vec{X}_{j}\right)=-\vec{v}\left(\vec{X}_{j}\right)+\overline{\vec{u}}_{j}, \\
& \overrightarrow{\vec{u}}_{j}=\left(c \mu^{2} / 4 \pi n q\right) \vec{A}_{p}\left(\vec{X}_{j}\right) .
\end{aligned}
$$

Thus $\overline{L_{X}\left(t_{0}\right)}$ is given by the equality

$$
\begin{aligned}
& \overline{L_{X}\left(t_{0}\right)}=\frac{m}{2} \sum_{j=1}^{N}\left(-\vec{v}^{2}\left(\vec{X}_{j}\right)+\frac{\mu^{2} c}{4 \pi n q} \vec{v}\left(\vec{X}_{j}\right) \cdot \vec{A}_{p}\left(\vec{X}_{j}\right)+\frac{q}{n c}\left(\frac{\mu^{2} c^{2}}{4 \pi n q^{2}}+\frac{2}{m}\right) \vec{v}\left(\vec{X}_{j}\right) \cdot \vec{A}_{p}\left(\vec{X}_{j}\right)\right) n \Delta V_{j}+ \\
& +\frac{\lambda q}{2 c} \sum_{j=1}^{N} \overline{\vec{u}_{j}} \cdot \vec{A}_{p}\left(\vec{X}_{j}\right) \Delta V_{j}= \\
& =\frac{m}{2} \sum_{j=1}^{N}\left(-\vec{v}^{2}\left(\vec{X}_{j}\right)+\frac{\mu^{2} c}{4 \pi n q} \vec{v}\left(\vec{X}_{j}\right) \cdot \vec{A}_{p}\left(\vec{X}_{j}\right)\right) n \Delta V_{j}+\frac{\lambda q}{2 c} \sum_{j=1}^{N}\left(-n \vec{v}\left(\vec{X}_{j}\right) \cdot \vec{A}_{p}\left(\vec{X}_{j}\right)+\frac{\mu^{2} c}{4 \pi q} \vec{A}_{p}^{2}\right) \Delta V_{j},
\end{aligned}
$$

where the relation (23.20) has been used in the second transition.

We consider arbitrary primary variations $\delta \vec{x}_{n}$ of the $\vec{x}_{n}\left(t_{0}\right)$. The $\delta \vec{x}_{n}$ produce a variation of the $\vec{X}_{j}\left(t_{0}\right)$ given by 


$$
\delta \vec{X}_{j}=\frac{1}{N_{j}} \sum_{n=1}^{N_{j}} \delta \vec{x}_{n}
$$

In turn the $\delta \vec{X}_{j}$ produce a variation of the $\vec{X}_{j}$-dependent quantities $\vec{v}\left(\vec{X}_{j}\right)$ and $\vec{A}_{p}\left(\vec{X}_{j}\right)$ ( $\vec{A}_{p}$ is treated as a given, albeit unspecified function of space)

$\delta \vec{v}\left(\vec{X}_{j}\right)=\delta \vec{X}_{j} \cdot \nabla \vec{v}\left(\vec{X}_{j}\right), \delta \vec{A}\left(\vec{X}_{j}\right)=\delta \vec{X}_{j} \cdot \nabla \vec{A}_{p}\left(\vec{X}_{j}\right)$

One obtains the following expression for the first variation of $\overline{L_{X}\left(t_{0}\right)}$ :

$\overline{\delta L_{X}}=\frac{m n}{2} \sum_{j=1}^{N} \Delta V_{j}\left(-2 \vec{v} \cdot \delta \vec{v}+\frac{\mu^{2} c}{4 \pi n q} \delta\left(\vec{v} \cdot \vec{A}_{p}\right)\right)+\frac{\lambda}{2} \sum_{j=1}^{N} \Delta V_{j}\left(-\frac{n q}{c} \delta\left(\vec{v} \cdot \vec{A}_{p}\right)+\frac{\mu^{2}}{2 \pi} \vec{A}_{p} \cdot \delta \vec{A}_{p}\right)$.

For comparison with the magnetic entropy it is convenient to express the variations in terms of the current density $\vec{j}\left(\vec{X}_{j}\right)=n q \vec{v}\left(\vec{X}_{j}\right)$ :

$$
\begin{aligned}
& \overline{\delta L_{X}}=\frac{m}{2 n q^{2}} \sum_{j=1}^{N} \Delta V_{j}\left(-2 \vec{j}\left(\vec{X}_{j}\right) \cdot \overrightarrow{\delta j}+\frac{\mu^{2} c}{4 \pi}\left(\vec{j}\left(\vec{X}_{j}\right) \cdot \delta \vec{A}+\vec{A}_{p}\left(\vec{X}_{j}\right) \cdot \overrightarrow{\delta j}\right)\right) \\
& -\lambda \sum_{j=1}^{N} \Delta V_{j}\left(\frac{1}{2 c}\left(\vec{j}\left(\vec{X}_{j}\right) \cdot \delta \vec{A}_{p}+\vec{A}_{p}\left(\vec{X}_{j}\right) \cdot \overrightarrow{\delta j}\right)-\frac{\mu^{2}}{4 \pi} \vec{A}_{p} \cdot \delta \vec{A}_{p}\right) .
\end{aligned}
$$

Thus, the variation of $\overrightarrow{\delta j}$, as well as of $\overrightarrow{A A}_{p}$, can be expressed in the form $\overrightarrow{\delta j}=\vec{\xi}_{j} \cdot \nabla \vec{j}\left(\vec{X}_{j}\right)$, $\delta \vec{A}_{p}=\vec{\xi}_{j} \cdot \nabla \vec{A}_{p}$, where $\vec{\xi}_{j}=\delta \vec{X}_{j}$ is a displacement field $\vec{\xi}_{j}=\vec{\xi}\left(\vec{X}_{j}\right)$. One can choose $\vec{\xi}\left(\vec{X}_{j}\right)$ according to physical requirements (for instance, incompressibility), or one can consider the variation $\overrightarrow{\delta j}\left(\vec{X}_{j}\right)$ as primary and determine $\vec{\xi}\left(\vec{X}_{j}\right)$ by solving the inhomogeneous linear system of equations $\overrightarrow{\delta j}\left(X_{j}\right)=\vec{\xi}_{j} \cdot \nabla \vec{j}\left(\vec{X}_{j}\right)$ and then choose a set of variations $\delta \vec{x}_{n}$ consistent with (24.5).

The two terms in the right hand side of (24.8) are related respectively to the first variation $\delta S_{p}$ of the magnetic entropy, and of the variation $\delta \Phi_{\text {int }}$ of the interaction energy, with respect to arbitrary primary variations $\overrightarrow{\delta j}\left(\vec{X}_{j}\right)$ or $\vec{\xi}\left(\vec{X}_{j}\right)$. The variation $\overline{\delta L_{X}\left(t_{0}\right)}$ then takes on the form

$\overline{\delta L_{X}\left(t_{0}\right)}=\Theta \delta S_{p}+\lambda \delta \Phi_{\text {int }}$,

with

$\Theta \delta S_{p}=\frac{m}{2 n q^{2}} \int_{\Omega} \vec{j} \cdot\left(\vec{j}-\frac{\mu^{2} c}{4 \pi} \vec{A}_{p}\right) \nabla \cdot \vec{\xi} d \Omega, \quad \lambda \delta \Phi_{\text {int }}=-\frac{\lambda}{2 c} \int_{\Omega} \vec{A}_{p} \cdot\left(\vec{j}-\frac{\mu^{2} c}{4 \pi} \vec{A}_{p}\right) \nabla \cdot \vec{\xi} d \Omega$,

where $\Theta=m \overline{\widetilde{V}^{2}} N_{j} / 3(\Theta=T$ in the case of the Maxwellian distribution of the single particle velocities, see (23.18)).

To show (24.10) we consider the equalities

$\sum_{j=1}^{N} \Delta V_{j} \vec{j}\left(\vec{X}_{j}\right) \vec{\xi} \cdot \nabla \vec{A}_{p}\left(\vec{X}_{j}\right)=\int_{\Omega} \vec{j} \vec{\xi} \cdot \nabla \vec{A}_{p} d \Omega=\int_{\Omega} \nabla \cdot\left(\vec{\xi}\left(\vec{j} \cdot \vec{A} \vec{A}_{p}\right)\right) d \Omega-\int_{\Omega} \vec{A}_{p} \cdot \vec{j} \nabla \cdot \vec{\xi} d \Omega$

$-\int_{\Omega} \vec{A}_{p}(\vec{\xi} \cdot \nabla \vec{j}) d \Omega$

where

$\int_{\Omega} \nabla \cdot\left(\vec{\xi}\left(\vec{j} \cdot \vec{A}_{p}\right)\right) d \Omega=\int_{\alpha \Omega}\left(\vec{j} \cdot \vec{A}_{p}\right) \vec{\xi} \times d \vec{S}$

We assume that the surface integral is vanishing either because $\vec{\xi} \times d \vec{S}=0$ or because, more stringently, $\vec{\xi}=0$, which implies $\overrightarrow{\delta j}=\delta \vec{A}_{p}=0$ on $\partial \Omega$, Then we obtain the relation 


$$
\int_{\Omega} \vec{j} \cdot \delta \vec{A}_{p} d \Omega+\int_{\Omega} \vec{A}_{p} \cdot \overrightarrow{\delta j} d \Omega=\int_{\Omega} \vec{j} \cdot \vec{A}_{p} \nabla \cdot \vec{\xi} d \Omega
$$

and similarly, the relations

$\int_{\Omega} \vec{A}_{p} \cdot \overrightarrow{\delta A} d \Omega=-\frac{1}{2} \int_{\Omega} \vec{A}_{p}^{2} \nabla \cdot \vec{\xi} d \Omega, \quad \int_{\Omega} \vec{j} \cdot \overrightarrow{\delta j} d \Omega=-\frac{1}{2} \int_{\Omega} \vec{j}^{2} \nabla \cdot \vec{\xi} d \Omega$

The equalities (24.10) follow from these relations and from (24.7).

Thus $\delta \overline{L_{X}\left(t_{0}\right)}=0$ leads to $\Theta \delta S_{p}+\lambda \delta \Phi_{\text {int }}=0$. This implies, recalling (24.10), that the plasma is incompressible, $\nabla \cdot \vec{\xi}=0$ or, for an arbitrary $\vec{\xi}$, that the integrand of $\Theta \delta S_{p}+\lambda \delta \Phi_{\text {int }}$ in (24.9) must vanish. In this case, defining $\vec{C}=\vec{j}-\left(\mu^{2} c / 4 \pi\right) \vec{A}_{p}$ the following equality must hold:

$\frac{m}{2 n q^{2}} \vec{j} \cdot \vec{C}-\frac{\lambda}{2 c} \vec{A}_{p} \cdot \vec{C}=0$.

We shall show that this equality is satisfied only by $\vec{C}=0$. Indeed, by eliminating $\vec{A}_{p}$ with the help of the definition of $\vec{C}$ one has

$\left(\frac{m \mu^{2} c^{2}}{4 \pi n q^{2}}-\lambda\right) \vec{j} \cdot \vec{C}+\lambda C^{2}=0$.

Recalling the considerations at the end of $\S 22$, the first term is negligible (recall eq. (23.22) and its physical interpretation) so that $\vec{C} \cdot(\vec{C}-\vec{j})=0$ and applying again the definition of $\vec{C}$ we can write $\vec{C} \cdot \vec{A}_{p}=0$ from which follows, using (24.15), that $\vec{C} \cdot \vec{j}=0$. Then (24.16) implies $C^{2}=0$.

As a consequence of this result the two terms (24.9) vanish separately, that is the condition $\overline{\delta L_{X}\left(t_{0}\right)}=0$ implies $\delta S_{p}=0, \delta \Phi_{\text {int }}=0$ and $\vec{A}_{p}$ must satisfy the equation $\nabla \times \nabla \times \vec{A}_{p}-\mu^{2} \vec{A}_{p}=0$.

At the same time one has from (24.3) and (23.5) that $\overline{\overrightarrow{\widetilde{V}}}=\Phi_{\text {int }}=0$, which is consistent with the fact (recalling the meaning of $\Phi_{\text {int }}$ ) that the unperturbed, as well as the perturbed collective system, are isolated.

Finally let us note the curious coincidence that, if one assumes that the canonical average $\overline{\delta L_{X}\left(t_{0}\right)}$ (where $L\left(t_{0}\right)=\tilde{L}_{p}\left(t_{0}\right)+L_{X}\left(t_{0}\right)$, see (24.1)) is equal to the time average over a long time interval $t_{2}-t_{1}$, $\overline{\delta L_{X}\left(t_{0}\right)}=\frac{1}{t_{2}-t_{1}} \int_{t_{1}}^{t_{2}} \delta L_{X}(t) d t$,

one would find that the Hamilton's action principle $\int_{t_{1}}^{t_{2}} \delta L(t) d t=0$ implies $\delta S_{p}=0$ in the limit $\left(l_{e} \mu\right)^{2}<<1$. Indeed, from (24.1a) one has (for isothermal variations, that is to say, by keeping fixed the sum of the kinetic energies of all the particles)

$\overline{\delta \tilde{L}_{p}\left(t_{0}\right)}=2 \delta \Phi_{\text {int }}$.

Combining (24.18) with (24.9) and (24.19) one obtains

$\frac{1}{t_{2}-t_{1}} \int_{t_{1}}^{t_{2}} \delta L(t) d t=\overline{\delta L_{X}\left(t_{0}\right)}+\overline{\delta \tilde{L}_{p}}=(2+\lambda) \delta \Phi_{\text {int }}+\Theta \delta S_{p} \rightarrow \Theta \delta S_{p}$

The term in the left hand side vanishes in view of the action principle and the equations of motion (22.12) and (22.13). It follows that in the limit $\left(l_{e} \mu\right)^{2}<<1$ or $\lambda \rightarrow-2$ one has $\delta S_{p}=0$. Conversely, 
applying the more restrictive condition $\overline{\delta L_{X}\left(t_{0}\right)}=0$, one has not only that $\delta S_{p}=0$, but also that $\delta \Phi_{\text {int }}=0$ as shown above, and then $\int_{t_{1}}^{t_{2}} \delta L(t) d t$ follows according to (24.20).

The primary variations considered here are the variations $\delta \vec{x}_{n}(t)$ (vanishing at the boundaries $t_{1}$ and $t_{2}$ ) of the positions of the particles in $\Delta V_{j}$ with respect to the positions $\vec{x}_{n}\left(t_{0}\right)$. The $\delta \vec{x}_{n}(t)$ produce the variation $\delta \vec{X}_{j}$ of the $\vec{X}_{j}$ according to the (24.5); $S_{p}$ and $\Phi_{\text {int }}$ are varied as above where the $\xi_{j}$ are interpreted as the time average of (24.5):

$$
\vec{\xi}_{j}=\frac{1}{t_{2}-t_{1}} \int_{t_{1}}^{t_{2}} \frac{1}{N_{j}} \sum_{n=1}^{N_{j}} \delta \vec{x}_{n}(t) d t
$$

The restriction to isothermal variations requires that $\sum_{n=11}^{N_{p}} \dot{\vec{x}}_{n}(t) \cdot \dot{\vec{x}} \dot{x}_{n}=0$. In view of the extremely large value of $N_{p}$ the arbitrariness in the choice of the $\delta \vec{x}_{n}(t)$ is practically unaffected by this condition.

The result above shows that, under the hypothesis (24.18), one has $\delta S_{p}=0$ as a consequence of the equations of motion (implicit in $\int_{t_{1}}^{t_{2}} \delta L(t) d t=0$ ) and therefore the entropy is conserved at first order. However the same does not hold for the second variation. This reflects the fact that the entropy is basically not a dynamical quantity, but an interpretative and predictive tool based on probability and information. Nevertheless we have seen in many examples (see chapters 2 and 3 ) that the negative second variation of the entropy is related to the positive second variation of the magnetic energy. This can be seen as a minimization of the energy under the optimization condition that the probability of the configuration be at a maximum.

\section{CHAPTER 8: Statistical model of electrostatic collisionless equilibria}

Summary: The statistical model developed in chapter 1 and applied there to magnetic equilibria is extended here to the case of collective electrostatic equilibria. The entropy functional and the other thermodynamic quantities are calculated with the procedure of chapter 1 , sections 2-5 by operating in the information space where the current density $\vec{J}_{j}=\vec{j}(\vec{x})+\overrightarrow{\tilde{j}}$ is replaced by the charge density $\sigma_{j}=\sigma\left(\vec{x}_{j}\right)+\tilde{\sigma}_{j}$ and the collective vector potential $\vec{A}\left(\vec{X}_{j}\right)$ is replaced by the electrostatic potential $\varphi\left(\vec{X}_{j}\right)$. The partition of the plasma volume into volume elements $\Delta V$ is such that the collective effects are visible at scale lengths much larger than $\Delta V$, where $(\Delta V)^{1 / 3}<\lambda_{D}\left(\lambda_{D}\right.$ is the Debye length).

One can consider reversible transformations in which electric charges and electrostatic energy are exchanged between the collective configuration and the fluctuating medium, which acts as a reservoir of electric charges. These transformations correspond to the magnetic transformations considered in section 9, with the exchange of electrostatic energy by means of exchange of electric charges, replacing the Poynting flux of electromagnetic energy.

The entropy change of the collective system defined by means of the reversible process above holds also when the medium is not considered or the collective system is isolated from the medium and undergoes an irreversible transformation with entropy increase. 
It will be shown that the minimum of the electrostatic entropy corresponds to the instability of the collective equilibrium predicted by Vlasov equation. Any distribution function with a single maximum is stable and corresponds to the maximum of the entropy. The Maxwellian case is contained consistently in the theory.

The electrostatic interaction energy with the medium can be expressed in a phenomenological way in terms of a dielectric constant that describes the reaction of the plasma, considered as a polarizable medium, to the presence of a collective charge. The plasma is unstable with respect to the variations of the charge density around the marginal point such that the linear dielectric constant becomes negative.

It will be shown that the total entropy $S=S_{p}+S_{b}$ is at a maximum with respect to variations of the bifurcation parameters (e.g. the temperature gradient) around the marginal point.

\section{Thermodynamic formalism for electrostatic collisionless equilibria}

The entropy pertaining to a collision-less electrostatic equilibrium can be constructed following the same procedure of chapter 1 after replacing the current density $\vec{J}_{j}=\vec{j}\left(\vec{x}_{j}\right)+\overrightarrow{\tilde{j}}_{j}$, averaged in the volume element $\Delta V_{j}$, with the charge density $\sigma_{j}=\sigma\left(\vec{x}_{j}\right)+\tilde{\sigma}_{j}$. The value of $\Delta V$ measures the coarsegraining through which the electrostatic collective equilibrium is seen: collective effects should be visible at the level larger than the coarse-graining described by $\Delta V$. This means that $(\Delta V)^{1 / 3}$ must be lower than the Debye length $\lambda_{D s}=\left(T_{s} / 4 \pi n_{s} q_{s}^{2}\right)^{1 / 2}$ of the species $s$ forming the plasma, and that particles in $\Delta V$ can be considered as effective free particles as shown by Pines and Bohm [2]. In addition $\Delta V$ must be large enough for containing many particles.

The collective charge density $\sigma\left(\vec{x}_{j}\right)$ is considered as superimposed on a background or medium of independent particles and the fluctuation of the number of uncorrelated particles contained in $\Delta V_{j}$ gives rise to the charge fluctuation $\tilde{\sigma}_{j}$. The electrostatic energy of the collective field is $\Phi=(1 / 2) \int_{\Omega} \sigma(\vec{x}) \varphi(\vec{x}) d \Omega$. We neglect the particle-particle correlation but consider instead the particlecollective field interaction described by the interaction energy $\Phi_{\text {int }}$ between the fluctuations $\tilde{\sigma}_{j}$ and the collective potential $\varphi(\vec{x})$. Just as in the magnetic case $\Phi_{\text {int }}$ will prove to be a useful device for describing the interaction of the collective system with the external sources of electrostatic energy and will acquire a definite physical meaning.

The probability distribution of the charge density $P\left(\sigma_{1} \ldots \sigma_{n} ; \vec{x}_{1} \ldots \vec{x}_{N}\right)$ is then constrained by the condition

$\frac{1}{2} \sum_{j=1}^{N} \int P\left(\sigma_{1} \ldots \sigma_{N} ; \vec{x}_{1} \ldots \vec{x}_{N}\right)\left(\sigma\left(\vec{x}_{j}\right)+\sigma_{j}\right) \varphi\left(\vec{x}_{j}\right) \Delta V_{j} d \Gamma=\Phi+\Phi_{\mathrm{int}}$,

where (similarly to (1.3)

$\Phi=\frac{1}{2} \sum_{j=1}^{N} \varphi\left(\vec{x}_{j}\right) \gamma\left(\vec{x}_{j}\right) \Delta V_{j}$,

and

$\Phi_{\text {int }}=\frac{1}{2} \int P\left(\sigma_{1} \ldots \sigma_{n} ; \vec{x}_{1} \ldots \vec{x}_{N}\right) \sum_{j=1}^{N} \tilde{\sigma}_{j} \varphi\left(\vec{x}_{j}\right) \Delta V_{j} d \Gamma$. 
Here $d \Gamma=\Delta V^{N} d \sigma_{1} \ldots d \sigma_{n} ; \Delta V=N / V$ measures the partition of a large volume $V$ of plasma, electrically neutral in the average, surrounding the collective charge distribution $\sigma(\vec{x})$ localized in a volume $\Omega<<$.

The vanishing of $\Phi_{\text {int }}$ characterizes a 'purely' collective equilibrium in the sense that the interaction with effects involving particle discreteness are ignored and only collective effects are considered. So, the condition $\Phi_{\text {int }}=0$ is the very definition of "Vlasov" equilibrium in our phenomenological picture. In fact we shall consider variations around the Vlasov equilibrium with $\delta \Phi_{\text {int }} \neq 0$ and this will describe the exchange of energy between the collective system and the medium associated with exchange of electric charges. So, in the presence of this interaction the system is no longer in the "pure Vlasov" equilibrium.

A further constraint arises naturally from the fact that the $\sigma_{j}$, in view of their fluctuating component $\tilde{\sigma}_{j}$, are random variables whose values are subject to dispersion. Similarly to eq.(2.7), we introduce the constraint

$\overline{\Delta \sigma^{2}}=\frac{1}{N} \int P\left(\sigma_{1} \ldots \sigma_{2} ; \vec{x}_{1} \ldots \vec{x}_{n}\right) \sum_{j=1}^{N}\left(\sigma_{j}\right)^{2} d \Gamma$.

The probability $P$ is calculated from the requirement that the entropy $S=-\int P \ln P d \Gamma$ be stationary with respect to variations of $P$ under the constraints (25.1), (25.4) and the normalization condition $\int P d \Gamma=1$. At this point we follow step by step the calculations of sections 3 and 4 and arrive at the relations

$P\left(\sigma_{1} \ldots \sigma_{N} ; \vec{x}_{1} \ldots \vec{x}_{N}\right)=\frac{\exp -\sum_{j=1}^{N} \alpha\left(\sigma_{j}-\overline{\sigma_{j}}\right)^{2}}{\Delta V^{N}(\pi / \alpha)^{N / 2}}=\frac{\exp -\sum_{j=1}^{N} \alpha\left(\tilde{\sigma}_{j}-\overline{\tilde{\sigma}_{j}}\right)^{2}}{\Delta V^{N}(\pi / \alpha)^{N / 2}}$,

where

$\overline{\sigma_{j}}=\sigma\left(\vec{x}_{j}\right)+\overline{\widetilde{\sigma}}_{j}=-\frac{1}{2 \alpha \tau} \varphi\left(\vec{x}_{j}\right) \Delta V_{j}$,

${\overline{\sigma_{j}^{2}}}^{2}-{\overline{\sigma_{j}}}^{2}=\frac{1}{2 \alpha}$,

$\overline{\Delta \sigma^{2}}=\frac{1}{N} \sum_{j=1}^{N} \sigma^{2}\left(\vec{x}_{j}\right)+\Delta \sigma_{c}+\frac{1}{N} \sum \overline{\tilde{\sigma}_{j}^{2}}$, where $\Delta \sigma_{c} \equiv \frac{2}{N} \sum_{j=1}^{N} \overline{\tilde{\sigma}_{j}} \sigma\left(\vec{x}_{j}\right)$.

After application of the thermodynamic limit $(V \rightarrow \infty, N \rightarrow \infty, \Delta V=$ const, $\Omega / V \rightarrow 0)$ one finds

$\tau=-\frac{1}{2} \Delta V \overline{\tilde{\sigma}^{2}} \frac{\int_{\Omega} \varphi^{2}(\vec{x}) d \Omega}{\Phi+\Phi_{\mathrm{int}}}$,

$\overline{\Delta \sigma^{2}}=\overline{\sigma_{j}^{2}}-{\overline{\sigma_{j}}}^{2}=\frac{1}{2 \alpha}=\overline{\tilde{\sigma}^{2}}$, where $\overline{\tilde{\sigma}^{2}}=\frac{1}{N} \sum_{j=1}^{N} \overline{\tilde{\sigma}_{j}^{2}}$.

The entropy $S$ is divided in a part $S_{p}$ pertaining to the collective system and in a part $S_{b}$, which contains the effects of the medium

$S=S_{p}+S_{b}$,

where

$S_{p}=\frac{N}{4 \overline{\tilde{\sigma}^{2}}} \Delta \sigma_{c}=\frac{1}{2 \Delta V \overline{\tilde{\sigma}^{2}}} \int_{\Omega} \sigma(x) \overline{\tilde{\sigma}}(x) d \Omega=-\frac{1}{2 \Delta V \overline{\tilde{\sigma}^{2}}}\left(\int_{\Omega} \sigma^{2}(\vec{x}) d \Omega+\frac{\Delta V \overline{\tilde{\sigma}^{2}}}{\tau} \int_{\Omega} \sigma(\vec{x}) \varphi(\vec{x}) d \Omega\right)$, 
$S_{b}=\frac{\Phi_{\mathrm{int}}}{\tau}=\frac{1}{2 \tau} \int_{\Omega} \varphi \overline{\tilde{\sigma}} d \Omega=-\frac{1}{2 \tau}\left(\int_{\Omega} \sigma(\vec{x}) \varphi(\vec{x}) d \Omega+\frac{\Delta V \overline{\tilde{\sigma}^{2}}}{\tau} \int_{\Omega} \varphi^{2}(\vec{x}) d \Omega\right)$.

The free energy is given by the same expression (4.8) as $F=-\tau S_{p}+\Phi$.

In the equalities above terms tending to infinity with $N$ have been neglected. For instance the exact form of $S_{b}$ would be

$S_{b}=\frac{\Phi_{\text {int }}}{\tau}+N \ln \left(\Delta V\left(2 \pi \overline{\tilde{\sigma}^{2}}\right)^{1 / 2}\right)+\frac{N}{2}$.

Indeed, one sees that these terms are independent of the quantities of the collective equilibrium and do not contribute to its variations.

\section{The electrostatic entropy concept}

We consider a neutral plasma perturbed by a single collective $k$ - mode $\sigma\left(\vec{x}, k_{i}, \omega\right)=(1 / \Omega)^{1 / 2} \sigma_{k} \exp (i \vec{k} \vec{x}-i \omega t)$ ( $k_{i}$ are the components of $\vec{k}$ ) and assume that a mode $\sigma_{m}\left(\vec{x}, k_{m, i}, \omega_{m}\right)$ exists which is marginally stable with real $\vec{k}_{m}, \omega_{m}$ so that a reference frame can be found where the mode is time independent. Thus, in this frame the mode describes a static electric equilibrium to which one can apply the concepts of canonical ensemble and of canonical $P$-average implied by our statistical procedure. We also assume that the equilibrium $\sigma_{m}$ corresponds to a vanishing interaction energy $\Phi_{\text {int }}=0$, in other words there is no electrostatic interaction of the collective system with the fluctuating medium and $\sigma_{m}$ is a pure collective mode in the sense of Vlasov. In general one can write $\sigma_{m}=\sigma_{m}\left(\vec{x}, k_{m, i}, \omega_{m}, \eta\right)$ where $\eta$ denotes the set of parameters that characterize the particular dynamical situation at hand, expressed by the specific form of the ion and density perturbations $\delta n_{i}, \delta n_{e}$ building up the electric charge density $\sigma=e\left(\delta n_{i}-\delta n_{e}\right)$. During the perturbation the parameter $\tau$ is kept fixed to its equilibrium value

$$
\tau_{m}=-\frac{1}{2} \Delta V \overline{\tilde{\sigma}^{2}} \frac{\int \varphi_{m}^{2} d \Omega}{\frac{1}{2} \int \sigma_{m} \varphi_{m} d \Omega}=-\frac{4 \pi \Delta V \overline{\tilde{\sigma}^{2}}}{k_{m}^{2}},
$$

where $k_{m}^{2} \varphi_{m}=4 \pi \sigma_{m}$. Then the variation functionals $S_{p}$ and $S_{b}$ take the form

$$
\begin{aligned}
& S_{p}=\frac{1}{2 \Delta V \overline{\tilde{\sigma}^{2}}}\left(-\int_{\Omega} \sigma^{2} d \Omega+\frac{k_{m}^{2}}{4 \pi} \int_{\Omega} \sigma \varphi d \Omega\right), \\
& S_{b}=\frac{\Phi_{\text {int }}}{\tau}=-\frac{1}{2 \tau} \int_{\Omega} \varphi\left(\sigma-\frac{k_{m}^{2}}{4 \pi} \varphi\right) d \Omega=\frac{1}{2 \tau} \int_{\Omega} \varphi \overline{\tilde{\sigma}} d \Omega,
\end{aligned}
$$

where $\sigma=\sigma_{m}+\delta \sigma$ and $\varphi=\varphi_{m}+\delta \varphi$ and use has been made of (25.6), (25.7), (25.10) and (26.1).

It will be shown that $\delta S_{p}=0$, provided the total collective charge is conserved in the variation. Indeed, let us start from the first variation of $S_{p}$ :

$$
\delta S_{p}=\frac{1}{2 \Delta V \overline{\tilde{\sigma}^{2}}}\left(-2 \int \sigma_{m} \delta \sigma d \Omega+\frac{k_{m}^{2}}{4 \pi} \int\left(\varphi_{m} \delta \sigma+\sigma_{m} \delta \varphi\right) d \Omega\right) .
$$

The application of the Green's theorem gives 


$$
\begin{aligned}
& \int \sigma_{m} \delta \varphi d \Omega=-\frac{1}{4 \pi} \int_{\Omega}\left(\nabla^{2} \varphi_{m}\right) \delta \varphi d \Omega= \\
& -\frac{1}{4 \pi} \int\left(\nabla^{2} \delta \varphi\right) \varphi_{m} d \Omega-\frac{1}{4 \pi} \int_{\partial \Omega}\left(\delta \varphi \nabla \varphi_{m}-\varphi_{m} \nabla \delta \varphi\right) \cdot d \vec{S} .
\end{aligned}
$$

We assume that the boundary surface $\partial \Omega$ is equipotential, thus $\delta \varphi=0$ and $\varphi_{m}=\varphi_{S}=$ const on $\partial \Omega$. Substitution of (26.5) into (26.4) and use of the Poisson equation for the electric field leads to the expression

$$
\begin{aligned}
& \delta S_{p}=\frac{1}{2 \Delta V \overline{\tilde{\sigma}^{2}}}\left(-\int_{\Omega} \sigma_{m} \delta \sigma d \Omega+\frac{k_{m}^{2}}{4 \pi} \int_{\Omega} \varphi_{m} \delta \sigma d \Omega-\frac{\varphi_{S}}{4 \pi} \int_{\Omega} \delta \vec{E} \cdot d \vec{S}\right)= \\
& -\frac{k_{m}^{2}}{32 \pi^{2} \Delta V \overline{\tilde{\sigma}^{2}}} \varphi_{S} \int_{\Omega} \nabla \cdot \delta \vec{E} d \Omega=\frac{\varphi_{S}}{2 \tau_{m}} \int_{\Omega} \delta \sigma d \Omega,
\end{aligned}
$$

and $\delta S_{p}=0$ follows from $\int \delta \sigma d \Omega=0$ (we exclude the case $\varphi_{S}=0$, so that the result does not depend on the arbitrary choice of the reference potential). One also finds that $\delta S_{p}=-\delta S_{b}=-\delta \Phi_{\text {int }} / \tau_{m}=0$ with a similar calculation. This means that there is no exchange of electric charges and of entropy between the collective subsystem and the medium and that the entropy $S=S_{p}+S_{b}$ of the total system is unchanged at first order. In the case $\int \delta \sigma d \Omega \neq 0$ the relation $\delta S_{p}=-\delta S_{b}$, still holds with $\delta S_{p} \neq 0$, apart from the adiabatic variations $\delta \varphi=\left(4 \pi / k_{m}^{2}\right) \delta \sigma$ with respect to which $\delta S_{p}=\delta S_{b}=0$ and the variation of the collective charge is zero. Indeed one can verify that the first variations of $S_{p}(26.2)$ and of $S_{b}(26.3)$ with respect to primary variations $\delta \varphi=\left(4 \pi / k^{2}\right) \delta \sigma$ are related as follows:

$$
\delta S_{p}=\frac{1}{2 \tau} \int_{\Omega} \varphi_{m}\left(1-\frac{k_{m}^{2}}{k^{2}}\right) \delta \sigma d \Omega=-\delta S_{b}=-\frac{\delta \Phi_{\mathrm{int}}}{\tau} .
$$

In this case the collective subsystem is not isolated but exchanges entropy and electric charges with the medium. We shall return to this point in section 27.

Let us consider the second variations of $S_{p}$ and of $S_{b}$,

$$
\delta^{2} S_{p}=-\frac{1}{2 \Delta V \overline{\tilde{\sigma}^{2}}} \int\left(1-\frac{k_{m}^{2}}{k^{2}}\right)(\delta \sigma)^{2} d \Omega \text {, and } \delta^{2} S_{b}=-\frac{k_{m}^{2}}{k^{2}} \int\left(1-\frac{k_{m}^{2}}{k^{2}}\right)(\delta \sigma)^{2} d \Omega \text {. }
$$

These relations hold independently of the value of the total charge variation $\int \delta \sigma d \Omega$. One has (summing up (26.2) and (26.3))

$$
\delta^{2} S=\delta^{2}\left(S_{p}+S_{b}\right)=-\frac{1}{2 \Delta V \overline{\tilde{\sigma}^{2}}} \int\left(1-\frac{k_{m}^{2}}{k^{2}}\right)^{2}(\delta \sigma)^{2} d \Omega \leq 0 .
$$

The entropy of the total system is at a maximum (the adiabatic variations apart) and the total system is therefore globally stable. The collective subsystem, at the contrary, can be stable or not in its interaction with the medium depending on the sign of $\delta^{2} S_{p}$.

It is essential to note that the entropy change of the collective subsystem holds also in the case where a medium is not considered and the collective subsystem is isolated and undergoes an irreversible transformation with entropy increase, while the same amount of energy $-\delta \Phi_{\text {int }}$ (see (26.7)), exchanged in an equivalent reversible process with the medium, is transformed and eventually dissipated internally to the subsystem. This irreversible process is associated with the same second variation of the collective entropy $\delta^{2} S_{p}$ as that given by (26.8). The sign of $\delta^{2} S_{p}$ establishes a 
direction in the evolution of the collective subsystem, namely the collective system evolves, when isolated, only along directions such that $\delta^{2} S_{p}>0$. Therefore $\delta^{2} S_{p}<0$ implies stability and $\delta^{2} S_{p}>0$ instability.

One sees from (26.8) that the unstable situations occur for the modes with $k^{2}<k_{m}^{2}$. We shall see in the next paragraphs that these thermodynamically unstable modes are associated with reactive (non dissipative) instabilities. For example, the reactive (non-resonant) modes predicted unstable by the dispersion function of the Vlasov equation associated with symmetric distribution functions in velocity space that are the sum of two Maxwellians centered at displaced velocities $\pm v_{0}$ whose difference is sufficiently large and the minimum of the distribution function sufficiently deep. This is the case of the two-stream instability.

Any distribution function with a single maximum is stable according to the Vlasov equation and corresponds to $k_{m}^{2}<0, \delta^{2} S_{p}<0$ and to a maximum of the electrostatic entropy. Detailed examples of stable electrostatic equilibrium and unstable situations associated with $k^{2}<k_{m}^{2}$ are discussed e.g. by Bernstein et al. [30].

For illustration's sake of the observations above let us consider the simple case of the electrostatic equilibrium described by the one-dimensional Vlasov equation, static in the laboratory frame of reference

$v \frac{\partial_{s}}{\partial x}+\frac{q_{s}}{m_{s}} E(x) \frac{\partial_{s}}{\partial v}=0$,

where $\frac{\partial E}{\partial x}=\sum_{s} 4 \pi q_{s} \int f_{s} d v$.

Combining the two equations above one readily derives an equation for the electric field

$\frac{d^{2} E}{d x^{2}}+k_{m}^{2}(x) E=0$,

where $k_{m}^{2}(x)=\sum_{s} \frac{4 \pi q_{s}^{2}}{m_{s}} \int \frac{\partial_{s}}{v \partial v} d v$.

In the Maxwellian case one has

$k_{m}^{2}=-4 \pi \sum_{s} \frac{q_{s}^{2} n_{s}}{T_{s}}<0$

The entropy $S_{p}$ is at a maximum and $-k_{m}^{2}$ is the sum of the inverse square of the Debye lengths of all species. In this case the equation (26.12) does not admit oscillatory solutions.

An oscillatory one-dimensional static solution of the electrostatic Vlasov equation is always associated with a non -Maxwellian distribution function and with a positive $k_{m}^{2}$.

The solution of (26.10) must be a function of the energy, namely $f_{s}=f_{s}\left(\xi_{s}\right)$ where $\xi_{s}=(1 / 2) m_{s} v^{2}+q_{s} \varphi$. Thus (26.13) becomes

$k_{m}^{2}=\sum_{s} 4 \pi q_{s}^{2} \int \frac{\partial_{s}}{\partial \xi_{s}} d^{3} v$.

The condition $\partial_{s} / \partial \xi_{s}>0$ in some domain of velocity space for at least one of the species, is necessary for $k_{m}^{2}>0$ and implies thermodynamic instability.

See the paper by Bernstein, Greene and Kruskal [32] for a nonlinear treatment of the solution of eqs. (26.10), (26-11), 
Recalling (26.1) and (26.14), in the Maxwellian case $\tau$ is given by the expression

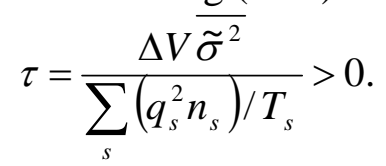

The mean square deviation in the number $\tilde{N}_{s}=\tilde{n}_{s} \Delta V$ of independent particles of species $s$ contained in $\Delta V$ is given by $\left({\overline{\tilde{n}_{s}^{2}}}^{-\tilde{n}_{s}}\right) \Delta V V^{2}=n_{s} \Delta V \quad$ (where $n_{s}=\overline{\tilde{n}}_{s}$ ) and the variance of the charge density of the system of uncorrelated particles with charge $q_{s}$ in $\Delta V$ will be $\overline{\tilde{\sigma}^{2}}=\sum_{s} q_{s}^{2}\left({\overline{\tilde{n}_{s}^{2}}}_{-\bar{n}_{s}^{2}}^{2}\right)=\sum_{s} \frac{q_{s}^{2} n_{s}}{\Delta V}$.

$$
\tau=\frac{\sum_{s}^{\text {Then, }} q_{s}^{2} n_{s}}{\sum_{s} q_{s}^{2} n_{s} / T_{s}} \text {. }
$$

When all species have the same temperature $T, \tau$ is identical with the Maxwellian temperature $T$. The Maxwellian case is then contained consistently in the present approach.

In the case of an inhomogeneous plasma one can define an electrostatic entropy functional which is sensible to the local structure of the equilibrium following step by step the same lines of section 11 for the magnetic case. Taking in mind eqs. (11.2), (11.3) one can write the following condition, which is sufficient for the minimum of the electrostatic entropy and for the linear thermodynamic instability of the one-dimensional Vlasov equilibrium

$\int_{\Delta x}(\varphi-\bar{\varphi})(\sigma-\bar{\sigma}) d x>0$,

where $\Delta x$ is the characteristic length of the equilibrium and $\bar{\varphi}, \bar{\sigma}$ are averages in $\Delta x$.

The criterion above has been compared with the results of numerical simulations in the case of selfconsistent inhomogeneous gravitational and electrostatic equilibria [32-34].

\section{Physical meaning of the electrostatic interaction energy}

The electrostatic interaction energy has a precise physical meaning, which we are going to illustrate. The first variation of $\Phi_{\text {int }}$ (26.3) around the equilibrium $\sigma_{m}=k_{m}^{2} \varphi_{m} / 4 \pi$ with respect to variations $\delta \varphi=\left(4 \pi / k^{2}\right) \delta \sigma$ has the form

$$
\delta \Phi_{\text {int }}=-\frac{1}{2} \int_{\Omega} \varphi_{m} \delta \sigma\left(1-\frac{k_{m}^{2}}{k^{2}}\right) d \Omega=\frac{1}{2} \int_{\Omega} \varphi_{m} \delta \overline{\tilde{\sigma}} d \Omega .
$$

As noted above (section 26) one has $\delta \Phi_{\text {int }}=-\tau_{m} \delta S_{p}$ and comparing (27.1) with (26.6) one finds that $\int \delta \sigma d \Omega \neq 0$ implies $k^{2} \neq k_{m}^{2}$ and $\delta \Phi_{\text {int }} \neq 0$. It follows that the variation of the total collective charge in $\Omega$ is accompanied by a perturbation $k^{2} \neq k_{m}^{2}$ away from marginal equilibrium. The value of $k_{m}^{2}$ will depend on the set of parameters $\eta$ that characterize the physical situation at hand around the marginal equilibrium and may depend also on the components $k_{m, i}$ of $\vec{k}$ as well as on the marginal frequency $\omega_{m}$, supposed to be real. Introducing the definition

$\varepsilon_{m}\left(k, k_{m, i} \omega_{m}, \eta\right)=1-\frac{k_{m}^{2}\left(k_{m, i}, \omega_{m}, \eta\right)}{k^{2}}$, 
the first variation of $\Phi_{\text {int }}$ becomes

$\delta \Phi_{\mathrm{int}}=-\frac{1}{2} \int \varphi_{m} \varepsilon_{m} \delta \sigma d \Omega=-\frac{1}{2} \int \varphi_{m} \delta \sigma_{e f f} d \Omega$,

where (see .(27.1))

$\delta \sigma_{\text {eff }} \equiv \varepsilon_{m} \delta \sigma=-\delta \overline{\tilde{\sigma}}$

is the effective charge which determines the amount of electrostatic energy transferred from the collective system to the medium (when $\delta \Phi_{\text {int }}>0$ ) or vice versa, in a perturbation that brings the system outside the marginal equilibrium. In this way $\varepsilon_{m}$ acquires the meaning of a dielectric constant and $\Phi_{\text {int }} \neq 0$ is the response of the polarizable medium, which reacts when a free charge distribution $\sigma\left(\vec{x}, k_{i}, \omega\right),\left(k \neq k_{m}\right)$ is introduced besides the preexisting collective charge distribution $\sigma_{m}=\sigma_{m}\left(\vec{x}, k_{m . i}, \omega_{m}, \eta\right)$ of the (initially) isolated $\left(\Phi_{\text {int }}=0\right)$ collective equilibrium. One can also say that the transition from the state $\sigma_{m}$ with $\Phi_{\text {int }}=0$ to the state $\sigma\left(\vec{x}, k_{i}, \omega\right),\left(k \neq k_{m}\right)$ with $\tau_{m}$ fixed and $\Phi_{\text {int }} \neq 0$, simulates the reaction of the medium to the (reversible) exchange of free charges $\sigma$ between the collective system and the external world (feigned by the medium).

In the absence of interaction one has from (27.3) that $\varepsilon_{m}\left(k_{m}, \omega_{m}, \eta\right)=0$, or $k^{2}=k_{m}^{2}\left(k_{m, i} \omega_{m}, \eta\right)$, which is the dispersion relation of the purely collective (Vlasov) mode $\sigma_{m}$.

This interpretation of the electrostatic interaction energy can also be seen from another point of view open to generalizations. The electrostatic equilibrium is characterized by a specific functional dependence (in general non-linear, see the examples in chapter 10) of the charge density on the potential $\sigma_{m}=\sigma_{m}\left(\varphi_{m}\right)$, which follows from the solution to the static Vlasov equation, involving the potential $\varphi_{m}$ through the constants of motion. A generic perturbation $\sigma(\vec{k}, \vec{x})$ of this equilibrium can be considered as formed by two parts

$\sigma=\sigma_{m}\left(\varphi_{m}+\varphi\right)-\sigma_{m}\left(\varphi_{m}\right)+\sigma_{\text {eff }}$,

where the first part is the adiabatic perturbation, which maintains the initial functional dependence $\sigma_{m}=\sigma_{m}\left(\varphi_{m}\right)$ of the zero-order equilibrium, and the second part $\sigma_{\text {eff }}$ describes the effective departure from this equilibrium. Let us expand (27.4) in powers of $\varphi$ obtaining up to first order

$\sigma=\frac{d \sigma_{m}}{d \varphi_{m}} \varphi+\sigma_{e f f}=\frac{k_{m}^{2}}{4 \pi} \varphi+\sigma_{e f f}$.

Substitution in (25.3) gives

$\Phi_{\text {int }}=-\frac{1}{2} \int \varphi \sigma_{\text {eff }} d \Omega$.

So $-\Phi_{\text {int }}$ can be interpreted as the electrostatic energy needed for driving the plasma outside the zero order adiabatic equilibrium specified by the function $\sigma_{m}=\sigma_{m}\left(\varphi_{m}\right)$.

This interpretation opens the question what effects the higher order terms of the expansion (26.5) could have on the reactive process considered above and what could be the plasma response to the non-linearity of the dielectric constant that arises from the higher order terms. We shall discuss this question in the next sections.

Let us proceed by considering the role played by the free energy $F$, defined by equation (25.14), in the charge exchange reactive process. The first variation of $F$ is given by the equality, recalling that $\delta S_{p}=-\delta S_{b}$ (see section 26):

$\delta F=-\tau_{m} \delta S_{p}+\delta \Phi=\delta \Phi_{\text {int }}+\delta \Phi=-\int \varphi_{m} \delta \sigma\left(\varepsilon_{m}-1\right) d \Omega$. 
The quantity $\delta \sigma\left(\varepsilon_{m}-1\right)$ is the polarization charge density of the medium. The polarizable medium is therefore the reserve of free energy in the unstable plasma with $\varepsilon_{m}-1 \propto-k_{m}^{2}<0$.

In the reversible process of charge exchange between the collective field and the medium one can put $\delta F=-\delta L$ where $\delta L$ is the work of the polarization charges in the potential $\varphi_{m}$.

The energy balance can be written in the form $\delta \Phi_{\text {int }}+\delta L=-\delta \Phi$.

Here $\delta \Phi_{\text {int }}>0$ is the energy input to the medium associated with the charge exchange with the collective system and $\delta L$ is the work spent by the polarization charges of the medium. The two terms are compensated by the variation $\delta \Phi$ of the electrostatic energy of the collective system.

We end this section by noting a relevant variation property of the total entropy $S=S_{p}+S_{b}$ that emerges when $S$ is expressed in terms of the dielectric constant $\varepsilon_{m}$ :

$S=-\frac{1}{2 \Delta V \overline{\tilde{\sigma}^{2}}} \int \sigma^{2} \varepsilon_{m}^{2} d \Omega$

We assume that a critical value $\eta=\eta_{m}$ of the parameters exists which corresponds to a bifurcation point of stability, namely

$\varepsilon_{m}\left(\vec{k}_{m}, \omega_{m}, \eta_{m}\right)=0, \frac{\partial S}{\partial \omega_{m}}=\frac{\partial \varepsilon_{m}\left(\vec{k}_{m}, \omega_{m}, \eta_{m}\right)}{\partial \omega_{m}}=0$,

where all quantities are real. One sees that $S$ is at a maximum with respect to arbitrary variations $\eta=\eta_{m}+\delta \eta$ around the marginal point $\eta_{m}$. Indeed the first and the second variations of $S$ (27.9) are

$\delta S=-\frac{1}{\Delta V \overline{\tilde{\sigma}^{2}}} \int \sigma^{2} \varepsilon_{m} \frac{\partial \varepsilon_{m}}{\partial \eta} \delta \eta d \Omega=0, \delta^{2} S=-\frac{1}{\Delta V \overline{\tilde{\sigma}^{2}}} \int \sigma^{2}\left(\frac{\partial \varepsilon_{m}}{\partial \eta} \delta \eta\right)^{2} d \Omega<0$.

The global entropy $S$ contains the contribution of the medium and is sensible to the reactive effect of the dielectric constant of the plasma as a whole, that is to say, the plasma including, at the appropriate scale lengths, the collective electric field and the effective free particles which act as a polarizable medium. In fact, the first variations of $S_{p}$ and $S_{b}$ do not vanish separately with respect to non adiabatic variations, while the global system with entropy $S=S_{p}+S_{b}$ can be at the thermodynamic equilibrium described by $\delta S=0$. Therefore one should expect that in a reactive (nondissipative) instability, generated by perturbation of $\eta$ around the marginal point, the plasma would set itself at the critical value $\eta=\eta_{m}$ where $S$ is at a maximum. For instance, in the case of the temperature gradient instability, it should set at the critical value of the temperature gradient.

\section{CHAPTER 9: Non-linear effects in reactive instabilities}

Summary: In the case of unstable plasmas and of non-Maxwellian velocity distributions the charge density cannot be separated in a collective component and in an effective free particle fluctuating component. The separation or screening distance described by the Debye length is a concept that holds only for thermal plasmas and Maxwellian distributions. We consider a model according to which the plasma with a negative dielectric constant on the unstable side of the marginal point reacts as a whole to the instability, without distinguishing in the nonlinear domain between collective and free particle effects, by providing promptly free electric charges for counteracting the collective instability as soon as the marginal point is crossed. In this way the whole plasma acts as a dielectric medium with an 
effective non-linear dielectric constant, whose non-linear terms are naturally the higher order terms of the expansion (26.5), which defines the effective departure $\sigma_{\text {eff }}$ from Vlasov equilibrium.

When second order terms in $\varphi$ are taken into account in the expansion of $\sigma_{m}\left(\varphi_{m}+\varphi\right)$, a term of fourth order appears in the expression of the entropy $S_{p}$, appropriate for the description of the electrostatic equilibrium in a nonlinearly reactive medium. One finds that for $\varepsilon_{m}=1-\left(k_{m} / k\right)^{2}<0$ the non-linear entropy is minimum for $\sigma=0$ and is at a maximum for a definite value of $\sigma^{2}$ with respect to variations of the charge density. So, for $\varepsilon_{m}<0$ the homogeneous equilibrium, when considered as isolated, is thermodynamically unstable, but the system builds up a neighboring equilibrium with a charge density $\sigma=k^{2} \varphi / 4 \pi$ that stabilizes non-linearly at a definite value of $\sigma^{2}$ that will be calculated. A further maximization of $S_{p}$ determines the most probable value of the wave- number $k$.

In a different point of view, one introduces the probability distribution of the amplitude fluctuations of $\varphi$ around the marginal point according to the relation $\Delta \Gamma=e^{S_{p}(\varphi)}$ where $\Delta \Gamma$ is the volume element in $\varphi$-space accessible to the subsystem with entropy $S_{p}(\varphi)$. One has an intrinsic background of thermodynamic fluctuations extending from thermal fluctuations of Maxwellian plasmas in the stable side of the marginal point to fluctuations at the marginal point of stability and in the linearly unstable side of this point. On this side one finds fluctuations whose mean square amplitude are equal to the corresponding values associated with the neighboring equilibrium considered above.

The thermodynamic fluctuations can be the seed for the excitation of collective modes that can eventually evolve dynamically and reach a saturated value as a consequence of the dynamic interaction between collective modes. These purely collective fluctuations should not be confused with the thermodynamic fluctuations considered above.

\section{The non-linear dielectric constant}

The separation of the plasma charge density in the collective particle component and in the effective free-particle fluctuating component (formed by particles moving freely with their comoving clouds created by Debye shielding), which is relevant only at distances below the Debye length, has been pointed out in the pioneering paper by Pines and Bohm quoted above. In our picture the concept of separation has been applied and extended by considering a collective (macroscopic) equilibrium (described by a static solution of Vlasov equation) on a space scale separated and screened from the fluctuating medium of effective free particles by the Debye length, but susceptible of exchanging energy with it. Indeed the introduction of the interaction energy $\Phi_{\text {int }}$ of the collective system with the fluctuating infinite medium involving particle discreteness, has been proved to be a useful device for describing the reversible exchange of electric charges and energy of the collective system with external sources and for defining the entropy changes through a reversible process. However, when considering unstable collective equilibria (with $k_{m}^{2}>0$, see section 26) we are faced with the disturbing fact that the screening effect described by the Debye length vanishes in the description based on the Vlasov distribution function. Indeed the Debye length follows from the Maxwellian form of the distribution function (as we have seen in section 26) while the distribution function in unstable equilibria may be very far from the Maxwellian and this is true even in the simple case of a static oscillatory solution of the one dimensional Vlasov equation for the electrostatic case (section 26). In contrast, in the 
Maxwellian plasma, the Debye length characterizes damped solutions of the equation (26.11) and these solutions describe the screening by the plasma of an externally applied static electric field. The crucial point is that, while the Debye length enters the dispersion relation of unstable plasmas through the negligible term $k^{2} \lambda_{D}^{2}<<1$, it ceases to describe any screening effect. Therefore the separation of collective and free particle effects based on a screening characteristic length as the Debye length, disappears in the case of the non-Maxwellian equilibrium. In fact the value of $-k_{m}^{2}>0$ which is equal to the Debye length in the Maxwellian case and implies damped solutions of eq.(26.11), shielding a static external electric field, becomes even negative in the unstable plasma, which implies oscillatory solutions throughout the plasma volume. As a consequence we are led to think that collective and individual effects not only cannot be separated in certain unstable situations, but that the interaction between them could play a basic role on the evolution of the instability. In fact, as we have noted above, when the electrostatic potential becomes large, higher order terms in the expansion (27.5) cannot be neglected and this could modify greatly the reactive process considered in section 27, leading possibly to a saturation of the instability in a state of higher electrostatic entropy.

In case we pursue this point of view, we have to admit that the interaction energy $\Phi_{\text {int }}$ is not merely a useful device for simulating charges and energy exchanges with external sources, but that the reactive process described by $\Phi_{\text {int }}$ has a phenomenological reality in which the plasma itself acts as a polarizable medium. That is, electric charges are promptly available in the plasma for reacting to the instability of the collective equilibrium as soon as the marginal point is crossed, and possibly for quenching it. This is of course a conjecture and the purpose of the next paragraphs is to develop its consequences to the point of calculating the thermodynamic fluctuation levels around a state saturated according to the mechanism outlined above, so as to reach a basis for comparison with the observations.

Clearly, the conjectured mechanism of saturation is basically different from that usually considered in the non-linear treatment of the collective instabilities based on Vlasov equation. In this treatment one considers the dynamic interaction between many k-modes that are purely collective. At the contrary in the present approach we consider the reaction of the plasma to one single mode and the saturation of the mode is due to the (supposed) reactive dielectric properties of the polarizable plasma as $a$ whole, that is, to its ability to provide free charges from the reservoir of effective free particles for counteracting the instability and reach a stable state of increased electrostatic entropy. In a fundamental kinetic approach this would mean that the backlash of the growing collective electric field in a reactive (non dissipative) instability, on the hierarchy of the particle-particle correlation terms in the exact Liouville equation, cannot be neglected, a fact that invalidates the purely collective description of the Vlasov model.

We proceed according to our phenomenological scheme and start with a single k-mode that we now write in the form:

$\sigma=\frac{1}{\Omega^{1 / 2}}\left(\sigma_{1} \cos \vec{k} \cdot \vec{x}+\sigma_{2} \sin \vec{k} \cdot \vec{x}\right)$

The nonlinear dielectric constant $\varepsilon_{n l}$ follows from a straightforward generalization of (27.5)

$$
\sigma=\sigma_{m}\left(\varphi_{m}+\varphi\right)-\sigma_{m}\left(\varphi_{m}\right)+\sigma_{e f f}=\frac{1}{4 \pi} \sum_{n=1}^{\infty} k_{m, n}^{2} \frac{\varphi^{n}}{n !}+\sigma_{e f f}
$$


where $k_{m, n}^{2}=4 \pi d^{n} \sigma_{m} / d \varphi_{m}^{n}, k_{m, 1}^{2}=k_{m}^{2}$ and $\sigma_{e f f}=\varepsilon_{n l} \sigma=\varepsilon_{n l} k^{2} \varphi / 4 \pi$. Thus

$\varepsilon_{n l}=1-\frac{1}{k^{2} \varphi} \sum_{n=1}^{\infty} k_{m, n}^{2} \frac{\varphi^{n}}{n !}$.

The interaction energy is now written as in (27.6) but with the dielectric constant $\varepsilon_{m}$ (27.2) replaced by $\varepsilon_{n l}$ :

$\Phi_{\mathrm{int}}=-\frac{1}{2} \int_{\Omega} \varepsilon_{n l} \sigma \varphi d V=-\frac{1}{2} \int_{\Omega}\left(\sigma-\frac{1}{4 \pi} \sum_{n=1}^{\infty} k_{m \cdot n}^{2} \frac{\varphi^{n}}{n !}\right) \varphi d V$.

In the stable case $\left(k_{m}^{2} \equiv k_{m, 1}^{2}<0\right) \Phi_{\text {int }}$ is finite at first order in $\varphi$ while in the unstable case $\Phi_{\text {int }}$ vanishes at the marginal point (section 27) and the first non-vanishing term is of order $\varphi^{2}$. In the following we shall study the effects of this term and we shall see that they are considerable.

It will be useful to observe that the expression above for $\Phi_{\text {int }}$ can be obtained from (25.13) with the substitution

$\sigma \rightarrow \sigma_{n l} \equiv \sigma-\frac{1}{4 \pi} \sum_{n=2}^{\infty} k_{m, n}^{2} \frac{\varphi^{n}}{n !}=\left(d \sigma_{m} / d \varphi_{m}\right) \varphi+\sigma_{e f f}$.

The observation above gives us the key for constructing the entropy of a nonlinear electrostatic equilibrium in a nonlinearly reactive medium in accordance with our statistical model. Indeed the statistical formalism outlined in section 25 is unchanged when the collective charge density $\sigma(\vec{x})$ is replaced by the nonlinear charge density $\sigma_{n l}(\vec{x})$. After this choice of the information variable, the formalism of section 25 leads directly to the interaction energy (28.4) and to the following expression for the part of the entropy pertaining to the collective equilibrium (compare with (25.12) or with (26.2)):

$S_{p}=-\frac{1}{2 \overline{\tilde{\sigma}^{2}} \Delta V}\left(\int_{\Omega} \sigma_{n l}^{2} d \Omega-\frac{k_{m}^{2}}{4 \pi} \int_{\Omega} \sigma_{n l} \varphi d \Omega\right) \approx-\frac{k^{4}}{32 \pi^{2} \overline{\tilde{\sigma}^{2}} \Delta V} \int_{\Omega}\left(\varphi^{2} \varepsilon_{m}+\frac{\varphi^{4}}{4\left(k / k_{m, 2}\right)^{4}}\right) d \Omega$,

where only terms up to second order in $\varphi$ of the expansion of $\sigma_{m}\left(\varphi_{m}+\varphi\right)$ were retained in the second transition (odd powers of $\varphi$ average to zero in the volume integration). In this approximation the part $S_{b}$ of the entropy involving the interaction with the medium remains the same as in the linear case (compare with (25.3) where $\tau$, fixed at the quasi-homogeneous unperturbed equilibrium $\sigma_{m}\left(\varphi_{m}\right)$, is given by (26.1)):

$S_{b}=\frac{\Phi_{\text {int }}}{\tau}=\frac{k_{m}^{2}}{8 \pi \overline{\tilde{\sigma}^{2}} \Delta V} \int_{\Omega}\left(\sigma_{n l}+\frac{k_{m}^{2} \varphi}{4 \pi}\right) \varphi d \Omega=\frac{k^{2} k_{m}^{2}}{32 \pi^{2} \overline{\tilde{\sigma}^{2}} \Delta V} \int_{\Omega} \varepsilon_{m} \varphi^{2} d \Omega$.

\section{The nonlinear neighboring state}

In this section we shall investigate the variation properties of the nonlinear entropy (28.6), pertaining to the collective configuration. We introduce the mode (28.1) into (28.6) and perform the volume integration:

$S_{p}=-\frac{k^{4}}{64 \pi^{2} \overline{\tilde{\sigma}^{2}} \Delta V}\left(\varepsilon_{m}\left(\varphi_{1}^{2}+\varphi_{2}^{2}\right)+\frac{3}{16\left(k / k_{m, 2}\right)^{4} \Omega}\left(\varphi_{1}^{2}+\varphi_{2}^{2}\right)^{2}\right)$.

We look for the extreme of $S_{p}$ with respect to variations of $\varphi_{1}, \varphi_{2}$ : 


$$
\begin{aligned}
& \frac{\partial S_{p}}{\partial \varphi_{1}}=\varphi_{1}\left(2 \varepsilon_{m}+\frac{3}{4\left(k / k_{m, 2}\right)^{4} \Omega}\left(\varphi_{1}^{2}+\varphi_{2}^{2}\right)\right)=0, \\
& \frac{\partial S_{p}}{\partial \varphi_{2}}=\varphi_{2}\left(2 \varepsilon_{m}+\frac{3}{4\left(k / k_{m, 2}\right)^{4} \Omega}\left(\varphi_{1}^{2}+\varphi_{2}^{2}\right)\right)=0 .
\end{aligned}
$$

These equations admit the solution $\varphi_{1}=\varphi_{2}=0$, which corresponds to homogeneous plasma, and the solution

$\frac{1}{\Omega}\left(\varphi_{1}^{2}+\varphi_{2}^{2}\right)=-\frac{8}{3} \varepsilon_{m}\left(\frac{k}{k_{m, 2}}\right)^{4}$,

which exists only for $\varepsilon_{m}<0$ or $k^{2}<k_{m}^{2}$.

To see whether the solutions correspond to a minimum or a maximum of $S_{p}$ we calculate the second order derivatives:

$$
\begin{aligned}
& \left.\frac{\partial^{2} S_{p}}{\partial \varphi_{n}^{2}}=-\frac{k^{4}}{32 \pi^{2} \overline{\tilde{\sigma}^{2}} \Delta V}\left(\varepsilon_{m}+\frac{3}{8\left(k / k_{m, 2}\right)^{4} \Omega}\left(\varphi_{1}^{2}+\varphi_{2}^{2}+2 \varphi_{n}^{2}\right)\right), \quad(n=1,2)\right) \\
& \frac{\partial^{2} S_{p}}{\partial \varphi_{1} \partial \varphi_{2}}=-\frac{k_{m, 2}^{4}}{32 \pi^{2} \overline{\tilde{\sigma}^{2}} \Delta V} \frac{3}{4 \Omega} \varphi_{1} \varphi_{2} .
\end{aligned}
$$

The solution $\varphi_{1}=\varphi_{2}=0$ is a maximum of $S_{p}$ for $\varepsilon_{m}>0$ and a minimum for $\varepsilon_{m}<0$. In the latter case an isolated collective equilibrium, although homogeneous, is not expected to be stable. In contrast, the inhomogeneous equilibrium described by (29.3) is a maximum of $S_{p}$ as can be seen from the sign of the second variation:

$$
\begin{aligned}
& \delta^{2} S_{p}=\frac{1}{2} \frac{\partial^{2} S_{p}}{\partial \varphi_{1}^{2}}\left(\delta \varphi_{1}\right)^{2}+\frac{\partial^{2} S_{p}}{\partial \varphi_{1} \partial \varphi_{2}} \delta \varphi_{1} \delta \varphi_{2}+\frac{1}{2} \frac{\partial^{2} S_{p}}{\partial \varphi_{2}^{2}}\left(\delta \varphi_{2}\right)^{2}= \\
& =-\frac{3 k_{m, 2}^{4}}{256 \pi^{2} \tilde{\sigma}^{2} \Delta V} \frac{1}{\Omega}\left(\varphi_{1} \delta \varphi_{1}+\varphi_{2} \delta \varphi_{2}\right)^{2}<0 .
\end{aligned}
$$

Thus, for $\varepsilon_{m}<0$, the homogeneous equilibrium is unstable, but the system builds up a finite charge density, which stabilizes nonlinearly at the level (29.3).

The maximum value of $S_{p}$ corresponding to (29.3) is given by the inequality:

$$
S_{p, \max }=\frac{1}{48 \pi^{2} \overline{\tilde{\sigma}^{2}} \Delta V} \varepsilon_{m}^{2} \frac{k^{8}}{k_{m, 2}^{4}} \Omega .
$$

$S_{p \text { max }}$ can be maximized further with respect to $k$ :

$\frac{\partial S_{p, \text { max }}}{\partial k}=4 k^{3}\left(2 k^{4}-3 k^{2} k_{m}^{2}+k_{m}^{4}\right)=0$.

Besides the trivial solution $k=0$ one has the two solutions

$$
k_{1}^{2}=k_{m}^{2}, \varepsilon_{m}=0, \quad k_{2}^{2}=\frac{k_{m}^{2}}{2}, \varepsilon_{m}=-1 \text {. }
$$

The first solution corresponds to the marginal point and to a minimum of $S_{p \text {,max }}(k)$, while the second solution is an absolute maximum of $S_{p}$ corresponding to a large amplitude mode with a wave number $k_{2}$ away from the marginal point. This mode will be interpreted in section 30 as the manifestation on the average of large amplitude fluctuations of the marginal state towards the linearly unstable side of the marginal point. 


\section{Probability distribution of charge fluctuations around the marginal equilibrium}

The collective configuration can exchange electric charges with the medium and therefore the amplitude of the collective quantities is not fixed but fluctuates. In contrast the infinite medium, considered as an infinite reservoir of electric charges, remains practically undisturbed by the charge exchange (looking at the complete expression for $S_{b}$ (25.15), one can see that in the thermodynamic limit $N \rightarrow \infty$, a finite variation of $\Phi_{\text {int }}$ can be compensated by an infinitesimal variation of $\overline{\tilde{\sigma}^{2}}$ ). We wish to study the amplitude fluctuations of the charge density of the collective subsystem with entropy $S_{p}\left(\varphi_{1}, \varphi_{2}\right)$, (29.1). For this purpose we have to consider only the volume element $\Delta \Gamma$ in the $\varphi_{1}, \varphi_{2}$ space that is accessible to the electrostatic configuration of the subsystem with the entropy $S_{p}\left(\varphi_{1}, \varphi_{2}\right)$. We apply the well-known relation $\Delta \Gamma=e^{S_{p}\left(\varphi_{1}, \varphi_{2}\right)}$.

Then, the probability $p$ of finding the subsystem with the amplitude in $d \varphi_{1} d \varphi_{2}$ at $\varphi_{1}, \varphi_{2}$ is given by the expression (here we follow the same line as in ref. [34]):

$p d \varphi_{1} d \varphi_{2}=\frac{e^{S_{p}\left(\varphi_{1}, \varphi_{2}\right)}}{\iint e^{S_{p}\left(\varphi_{1}, \varphi_{2}\right)} d \varphi_{1} d \varphi_{2}}$.

We are interested in the mean square value $\overline{\varphi^{2}}=(1 / \Omega) \overline{\left(\varphi_{1}^{2}+\varphi_{2}^{2}\right)}$ of the potential. Recalling (29.1) and performing the change of variables $\varphi_{1}=\left(t-z^{2}\right)^{1 / 2}, \varphi_{2}=z$ with $d \varphi_{1} d \varphi_{2}=(1 / 2)\left(t-z^{2}\right)^{1 / 2} d z d t$ one has

$$
\overline{\varphi^{2}}=\frac{\iint\left(\varphi_{1}^{2}+\varphi_{2}^{2}\right) e^{S_{p}\left(\varphi_{1}, \varphi_{2}\right)} d \varphi_{1} d \varphi_{2}}{\Omega \iint e^{S_{p}\left(\varphi_{1}, \varphi_{2}\right)} d \varphi_{1} d \varphi_{2}}=\frac{\int_{0}^{\infty} t e^{\left(-\alpha t-\beta t^{2}\right)} d t}{\Omega \int_{0}^{\infty} e^{\left(-\alpha t-\beta t^{2}\right)} d t},
$$

where

$$
\alpha=\varepsilon_{m} \frac{k^{4}}{(8 \pi)^{2} \overline{\tilde{\sigma}^{2}} \Delta V}, \quad \beta=\frac{3 k_{m, 2}^{4}}{(32 \pi)^{2} \overline{\tilde{\sigma}^{2}} \Delta V} \frac{1}{\Omega} .
$$

Applying the equality $-\alpha t-\beta t^{2}=-\beta(t+\alpha / 2 \beta)+\alpha^{2} / 4 \beta$ the integrals in (30.3) can be expressed in terms of the error function as

$\overline{\varphi^{2}}=\frac{1}{4 \beta \Omega}\left(\frac{e^{\left(-\alpha^{2} / 4 \beta\right)}}{\operatorname{erfc}\left(\alpha / 2 \beta^{1 / 2}\right)}-\alpha\right)$,

where $\operatorname{erfc}(z)=\left(2 / \pi^{1 / 2}\right) \int_{z}^{\infty} \exp \left(-z^{2}\right) d z$.

One has three cases:

1)- $\varepsilon_{m}>0, \alpha>0$; noting that

$$
\frac{\alpha}{2 \beta^{1 / 2}}=\varepsilon_{m} \frac{k^{4}}{4 \pi k_{m, 2}^{4}\left(3 \Delta V \overline{\tilde{\sigma}^{2}}\right)^{1 / 2}} \Omega^{1 / 2}
$$

is a very large number because $\Omega$ is large, one can apply the asymptotic representation of the error function [36] 
$\operatorname{erfc}\left(\alpha / 2 \beta^{1 / 2}\right) \approx \frac{2}{\alpha}\left(\frac{\beta}{\pi}\right)^{1 / 2}\left(1-\frac{2 \beta}{\alpha^{2}}\right) e^{\left(-\alpha^{2} / 4 \beta\right)}$,

and obtain (recall (26.15)

$\overline{\varphi^{2}}=\frac{1}{\alpha \Omega}=\frac{64 \pi^{2} \overline{\tilde{\sigma}^{2}} \Delta V}{\varepsilon_{m} \Omega k^{4}}=\frac{64 \pi^{2}}{\varepsilon_{m} \Omega k^{4}} \sum_{s} q_{s}^{2} n_{s}$.

In the special case of a Maxwellian plasma with the temperature $T$ for all species one has $\varepsilon_{m}=1+1 / k^{2} \lambda_{D}^{2}$ where $\lambda_{D}^{-2}=4 \pi \sum_{s} q_{s}^{2} n_{s} / T$ (see (26.13) and

$\overline{\varphi^{2}}=\frac{16 \pi}{\Omega} \frac{T}{k^{2}\left(1+k^{2} \lambda_{D}^{2}\right)}$.

This relation is comparable with that derived by Taylor and McNamara [37] for the thermal fluctuations of a two dimensional plasma.

2)- $\varepsilon_{m}=0, \alpha=0$; this case concerns the fluctuations at the marginal point $\varepsilon_{m}=0$. The integrals in (30.5) are elementary and one obtains

$\overline{\varphi^{2}}=32\left(\frac{\pi}{3}\right)^{1 / 2}\left(\sum_{s} q_{s}^{2} n_{s}^{2} \frac{1}{N_{s}}\right)^{1 / 2}\left|k_{m, 2}^{-2}\right|$.

These are Poisson-like fluctuations whose mean square value depends on the inverse of the square root of the number of particles $N_{s}=n_{s} \Omega$.

3)- $\varepsilon_{m}<0, \alpha<0$; in this case $\alpha / 2 \beta^{1 / 2}$ tends to minus infinity and (30.5) becomes

$\overline{\varphi^{2}}=\frac{|\alpha|}{2 \beta \Omega}=\frac{8}{3} \mid \varepsilon_{m}\left(\frac{k}{k_{m, 2}}\right)^{4}$.

This is the same expression (29.3) for the square of the potential of the nonlinear neighboring equilibrium associated with a maximum of $S_{p}\left(\varphi_{1}, \varphi_{2}\right)$. This equilibrium is now interpreted as the average of large fluctuations in the region of negative $\varepsilon_{m}$ where the system is linearly unstable.

For the numerical evaluation of the fluctuation amplitude one must now calculate the coefficient $k_{m, 2}^{2}$ according to the expansion (28.2). In this connection it is worthwhile to recall that the equilibrium relation $\sigma_{m}\left(\varphi_{m}\right)$, which is at the base of (28.2), follows from the static solution of the Vlasov equation, which involves the potential $\varphi_{m}$ through the constants of motion. We assume that the zero order equilibrium is quasi-homogeneous, that is we shall consider the limit $\varphi_{m} \rightarrow 0$. This implies that for calculating the constants of motion, the linear approximation is sufficient. However the relation $\sigma_{m}\left(\varphi_{m}\right)$ is non-linear (see the examples in the next chapter) and the fluctuation $\varphi$ brings the system outside the Vlasov-Poisson equilibrium, from the collective charge density $\sigma_{m}$ to the charge density $\sigma=\sigma_{m}\left(\varphi_{m}+\varphi\right)-\sigma_{m}\left(\varphi_{m}\right)+\varepsilon_{n l} \sigma$, as we have noted in section 28. So the fluctuation $\varphi$, which can be much larger than $\varphi_{m}$, is not a solution to the Vlasov equation supplemented by Poisson's equation (the system of equations (26.9) and (26.10)). The probability of the fluctuation amplitude $\varphi$ around the Vlasov (collective) equilibrium $\varphi_{m}$ is given by (30.2) where the entropy $S_{p}$ is determined according to the non-linear reactive process described above, which is not contained in the Vlasov model. The probability distribution of the amplitude fluctuations (30.2) describes an intrinsic background of thermodynamic fluctuations near the stable side of the marginal point (thermal fluctuations) or near the linearly unstable side of this point. We shall see in section 35 how the electrostatic force arising from the fluctuating electric field can be compensated by fluctuations of the pressure. 
The picture above does not preclude at all the subsequent interactions of the basic fluctuation background with collective modes; at the contrary the thermodynamic fluctuations can even be the necessary seed for the excitation of collective modes. In a subsequent dynamic process the collective modes will eventually reach a saturated fluctuation level as a consequence of non-linear dynamic interactions between them. These purely collective fluctuations should not be confused with the intrinsic background of thermodynamic fluctuations considered above.

\section{CHAPTER 10: Thermodynamic fluctuation levels: examples}

Summary: The scheme outlined above for the calculation of the thermodynamic fluctuations can be applied to the large class of the so-called "reactive" instabilities characterized by absence of dissipation, algebraic dispersion relations (in the slab approximation) and existence of bifurcation points. This class includes unstable modes important for thermonuclear machines, as the ion or electron temperature gradient modes (ITG or ETG), the trapped electron modes (TEM), the flutes modes as well as instabilities not involving the magnetic field as the two-stream instability. A review of the reactive instabilities relevant for magnetic plasma confinement can be found in the book of Weiland [38].

In this chapter we shall limit ourselves to illustrate the method with two simple examples, namely the case of the two-stream instability where the distribution function depends on one constant of motion (the energy) and the case of the flute modes where the distribution function depends on two constants of motion. In the more physically interesting cases of the thermodynamic fluctuations of reactive drift modes one has a third constant related to the motion parallel to the magnetic field. We do not consider the drift modes here.

\section{Thermodynamic fluctuations in reactive purely electrostatic systems}

As observed in section 26, a typical example of reactive electrostatic instability is the two-stream instability that arises in the presence of two equal warm electron beams described by the onedimensional distribution function, symmetric in velocity space, formed by the sum of two Maxwellians centered at the velocities $\pm u$ respectively. A characteristic feature of this instability is that there is practically no dispersion, the linear dielectric constant $\varepsilon_{m}$ is negative in the unstable region and the phase velocity of the unstable waves is exactly zero

In accordance with the procedure of section 28 we start from the Vlasov stationary equilibrium characterized by the function $\sigma_{m}=\sigma_{m}\left(\varphi_{m}\right)$ defined by the equality

$\sigma_{m}\left(\varphi_{m}\right)=\sum_{s} q_{s} \int f_{s}\left(\xi_{s}\right) d v$

where $\xi_{s}=(1 / 2) m_{s} v^{2}+q_{s} \varphi_{m}$

We assume that the functions $f_{s}$ can be expanded in powers of $\varphi_{m}$ provided that $\varphi_{m}$ is sufficiently small. So one can also expand the function $\sigma_{m}\left(\varphi_{m}+\varphi\right)$ around a vanishing value of $\varphi_{m}$, obtaining up to second order

$4 \pi \sigma_{m}\left(\varphi_{m}+\varphi\right)=k_{m}^{2} \varphi+k_{m, 2}^{2}\left(\frac{\varphi^{2}}{2}\right)$. 
The coefficient $k_{m}^{2}$ becomes positive for values of $2 u$ above a critical value $2 u_{c}$ and the system becomes unstable for $k^{2}<k_{m}^{2}$.

Once the coefficients $k_{m}^{2}$ and $k_{m, 2}^{2}$ are known one can apply the formulas of section 30 for calculating the mean square amplitude of the thermodynamic fluctuations. On the unstable side of the marginal point this is given by eq. (29.3) or (30.11):

$\overline{\varphi^{2}}(k)=\frac{|\alpha|}{2 \beta \Omega}=\frac{8}{3}\left(\frac{k_{m}^{2}}{k^{2}}-1\right)\left(\frac{k}{k_{m, 2}}\right)^{4}$.

This expression is maximum for $k^{2}=k_{m}^{2} / 2$ and also, as shown in section 29 (eq.(29.8)), corresponds to an absolute sharp maximum of the entropy. Summing up with respect to the two signs of $\pm k$ one has that the most probable mean square value of $\varphi$ is given by the expression

$\left(\overline{\varphi^{2}}\right)_{M}=\frac{4}{3}\left(\frac{k_{m}}{k_{m .2}}\right)^{4}$,

which corresponds to the following mean square value of the electric field:

$\left(\overline{E^{2}}\right)_{M}=\frac{4}{3} \frac{k_{m}^{6}}{k_{m, 2}^{4}}$.

As a curiosity let us compare these results with the solution of the one-dimensional Poisson's equation with the charge density given by (31.2)

$-\frac{d^{2} \varphi}{d x^{2}}=k_{m}^{2} \varphi+\frac{1}{2} k_{m .2}^{2} \varphi^{2}$,

from which follows the first integral

$\left(\frac{d \varphi}{d x}\right)^{2}=-k_{m}^{2} \varphi^{2}-\frac{1}{3} k_{m, 2}^{2} \varphi^{3}+C \equiv F(\varphi)$

where $C$ is an integration constant. A second integration leads to the expression

$x-x_{1}=\int_{\varphi_{1}}^{\varphi} \frac{d \varphi}{(F(\varphi))^{1 / 2}}$.

We look for a periodic solution, regular everywhere for the real values of $x$. Then $F(\varphi)$ should admit another zero $F\left(\varphi_{2}\right)=0$ such that $F(\varphi)>0$ for $\varphi_{1}<\varphi<\varphi_{2}$. This occurs when [39]

$\Delta \equiv g_{2}^{3}-27 g_{3}^{2}<0$,

where $g_{2}=k_{m}^{4} / 12, g_{3}=\left(k_{m}^{6} / 6^{3}\right)-\left(k_{m, 2}^{4} C /(9 \cdot 16)\right)$.

The condition (31.9) is verified for

$\left(\frac{d \varphi}{d x}\right)_{M}^{2}=C \leq \frac{4}{3} \frac{k_{m}^{6}}{k_{m .2}^{2}}$,

where the subscript $M$ indicates the maximum value of $(d \varphi / d x)^{2}$. One has a family of solutions depending on $C$ whose squared electric field has the upper limit (31.10), which is the same as the maximum mean square value (31.5) of the electric field of the thermodynamic fluctuations. The solutions are expressed in terms of the Weierstrass function $\wp\left(x, g_{2} . g_{3}\right)$ as follows:

$\varphi-\varphi_{1}=\frac{1}{4} F^{\prime}\left(\varphi_{1}\right)\left(\wp\left(x-x_{1}, g_{2}, g_{3}\right)-\frac{1}{24} F^{\prime \prime}\left(\varphi_{1}\right)\right)^{-1}$. 
The solution oscillates with a wavelength $2 \omega / k_{m}$ (see [35]) where $\omega$ depends on $C$ and $\omega \rightarrow \pi$ for $C \rightarrow 0$ (linear limit). When $C$ increases the wavelength also increases, corresponding to the unstable modes $k^{2}<k_{m}^{2}$ of the thermodynamic treatment and to the non-linear region. However $\varphi$ oscillates between amplitudes $\varphi_{1}$ and $\varphi_{2}$ with opposite sign and with $\left|\varphi_{1}\right| \neq\left|\varphi_{2}\right|$. The amplitude of the oscillation is of the same order of (31.4) for $k^{2} \leq k_{m}^{2} / 2$ but it does not exhibit the sharp maximum of the thermodynamic theory predicted by (31.3) for $k^{2}=k_{m}^{2} / 2$. Instead it grows steadily tending to oscillate between $-2 k_{m}^{2} / k_{m, 2}^{2}$ and $k_{m}^{2} / k_{m, 2}^{2}$ while approaching the infinite wavelength for $C \rightarrow 4 / 3$.

\section{Thermodynamic fluctuations of flute modes}

\section{1-The constants of motion}

We consider a inhomogeneous plasma, but with uniform temperature $T_{e}=T_{i}=T$, situated in a constant magnetic field $\vec{B}=(0,0, B)$ and with ions and electrons subject to a gravitational force $m_{s} g_{s} \vec{e}_{x}$. The constant $g_{s}$ simulates the effects of curvature of the magnetic field, i.e. $g_{s}=v_{t h, s}^{2} / R_{c}$ where $R_{c}$ is the radius of curvature, $v_{t h, s}$ is the thermal velocity of species $s$ and $g_{e}=m_{i} g_{i} / m_{e}$. The gravitational force gives rise to a drift $v_{D s}=-g_{s} / \Omega_{s}$ where $\Omega_{s}=q_{s} B / m_{s} c$ is the cyclotron frequency. We consider modes $\varphi=\varphi_{k} \exp (i k y-i \omega t)$ and operate in a reference system $K^{\prime}$ moving with the phase velocity $\omega / k$ of the wave, so that $y^{\prime}=y-\omega t / k, v_{y}^{\prime}=v_{0 y}-\omega / k$ where $v_{0 y}$ is the velocity in the laboratory system.

We recall that the problem of calculating the function $\sigma_{m}=\sigma_{m}\left(\varphi_{m}\right)$ is a linear Vlasov problem because the $\sigma_{m}, \varphi_{m}$ are arbitrarily small. Therefore it is sufficient to calculate the equations for the orbits in linear approximation. These equations in drift approximation are given by the equalities

$$
\begin{array}{ll}
v_{x}^{\prime}=v_{\perp} \cos (\theta-\Omega s)+c \frac{E_{y}\left(y^{\prime}\right)}{B}, & x^{\prime}=-\frac{v_{\perp}}{\Omega} \sin (\theta-\Omega s)+\frac{c}{B} \int_{0}^{s} E_{y}\left(y^{\prime}\right) d s+\frac{v_{\perp}}{B} \sin \theta+x, \\
v_{y}^{\prime}=v_{\perp} \sin (\theta-\Omega s)+v_{D}-\frac{\omega}{k}, & y^{\prime}=\frac{v_{\perp}}{\Omega} \cos (\theta-\Omega s)+\left(v_{D}-\frac{\omega}{k}\right) s-\frac{v_{\perp}}{\Omega} \cos \theta+y .
\end{array}
$$

Here $\theta$ is the gyration angle and $|\Omega|>>\left|\omega-v_{D} k\right|$.

It can be verified that the quantity

$$
\chi(s) \equiv \chi\left(x^{\prime}(s), v_{y}^{\prime}(s), s\right)=\Omega x^{\prime}(s)+v_{y}^{\prime}(s)-\frac{q}{m} \int_{-\infty}^{s} E_{y}\left(y^{\prime}\right) d s
$$

satisfies the equality $d \chi / d s=0$ and therefore is a constant of the motion described by (32.1). The value of $\chi$ is obtained after introducing (32.1) in (32.2) and putting $s=0$,

$\chi=\Omega x+v_{y}+v_{D}-\frac{\omega}{k}-\frac{q}{m} \int_{-\infty}^{0} E_{y}\left(y^{\prime}\right) d s$.

We now consider the quantity 
$\xi(s) \equiv \xi\left(x^{\prime}(s), y^{\prime}(s), v_{x}^{\prime}(s), v_{y}^{\prime}(s)\right)=\frac{1}{2}\left(\left(v_{x}^{\prime}-c \frac{E_{y}\left(y^{\prime}\right)}{B}\right)^{2}+v_{y}^{\prime 2}\right)-x^{\prime}\left(g-\Omega \frac{\omega}{k}\right)+$

$\frac{q}{m c} \varphi_{k} \exp \left(i k y^{\prime}\right)$

By introducing the equations of the orbits (32.1) into (32.4) one obtains

$\xi=\frac{1}{2}\left(v_{x}^{2}+\left(v_{y}+v_{D}-\frac{\omega}{k}\right)^{2}\right)-g x-\frac{q B \omega}{m c k}+\frac{q}{m}\left(v_{D}-\frac{\omega}{k}\right) \int_{0}^{s} E_{y}\left(y^{\prime}\right) d s+\frac{q}{m} \varphi_{k} \exp i k y^{\prime}(s)$,

where $v_{x}=v_{x}^{\prime}(0), v_{y}=v_{y}^{\prime}(0), x=x^{\prime}(0), y=y^{\prime}(0)$.

For evaluating the integral we use the well-known relation

$\exp \left(i k y^{\prime}\right)=\exp \left(i k\left(y+\left(v_{D}-\frac{\omega}{k}\right) s\right)\right) \sum_{l, l^{\prime}=-\infty}^{\infty}(i)^{l}(-i)^{l^{\prime}} J_{l}\left(\frac{k v_{\perp}}{\Omega}\right) J_{l^{\prime}}\left(\frac{k v_{\perp}}{\Omega}\right) \exp (i l(\theta-\Omega s)) \exp \left(-i l^{\prime} \theta\right)$.

After averaging over the rapid oscillations at the cyclotron frequency $\Omega$ only the term $l=0$ contributes to the $l$-summation. It is then easy to show that

$\frac{q}{m}\left(v_{D}-\frac{\omega}{k}\right) \int_{0}^{s} E_{y}\left(y^{\prime}\right) d s+\frac{q}{m} \varphi_{k} \operatorname{expiky}(s)=\frac{q}{m} \varphi_{k}$ exp iky.

Thus

$\xi=\frac{1}{2}\left(v_{x}^{2}+\left(v_{y}+v_{D}-\frac{\omega}{k}\right)^{2}\right)-g x-\frac{q B \omega}{m c k}+\frac{q}{m} \varphi_{k} \exp i k y$

does not depend on $s$ and is a constant of motion.

With the help of (32.6) we can evaluate the integral in the expression for the constant of motion $\chi$ (32.3).

As above, only the term $l=0$ contributes to the integral

$\int_{-\infty}^{0} E_{y} d s=-i k \varphi_{k} \int_{-\infty}^{0} e^{i k y^{\prime}(s)} d s=-\frac{\varphi_{k}}{v_{D}-\omega / k} e^{i k y} J_{0}\left(\frac{k v_{\perp}}{\Omega}\right) \sum_{l^{\prime}}(-i)^{l^{\prime}} J_{l^{\prime}}\left(\frac{k v_{\perp}}{\Omega}\right) e^{-i l^{\prime} \theta}$.

(The integration involves the limit of $\exp \left(i s\left(v_{D}+\omega / k\right)\right.$ ) for $s \rightarrow-\infty$, which does not exist; multiplication of the integrand by the "measure" $\exp (\varepsilon s)$, where $\varepsilon$ is an arbitrarily small positive number, resolves the difficulty). In (32.9) the terms with $l, l^{\prime}>0$ can be neglected, as they will vanish after the subsequent integration in velocity space with respect to $\theta$. So the expression (32.3) for $\chi$ becomes

$\chi=\Omega x+v_{y}+v_{D}-\frac{\omega}{k}+\frac{q \varphi_{k} e^{i k y} J_{0}^{2}\left(k v_{\perp} / \Omega\right)}{m\left(v_{D}-\omega / k\right)}$.

\section{2-The form of the distribution function}

From the constancy of $\chi$ and $\xi$ it follows that any function $f(\chi(s), \xi(s))$ satisfies the Vlasov equation under the same approximations considered above,

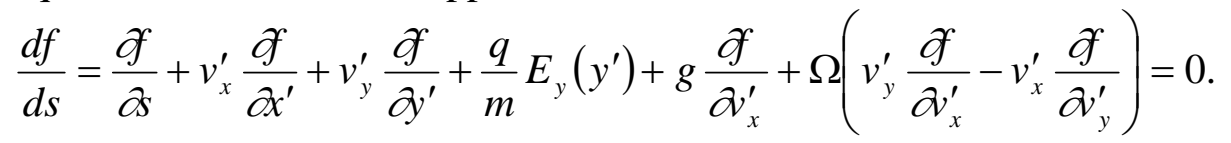


The functional dependence $f(\chi, \xi)$ is fixed by the requirement that $f$ be consistent, at zero order in $\varphi_{k}$, with the following expression (in the moving frame of reference $K^{\prime}$ )

$$
f_{0}\left(x^{\prime}, v_{x}^{\prime}, v_{y}^{\prime}\right)=N\left(1-\kappa x^{\prime}\right) \exp \left(-\alpha\left(v_{x}^{\prime 2}+\left(v_{y}^{\prime}+\frac{\omega}{k}\right)^{2}\right)\right) \text {, }
$$

where $\alpha=1 / v_{\text {th }}^{2}$ and $\kappa^{-1}$ measures the characteristic length of the density inhomogeneity. Then $f$ is fixed as

$$
f(\chi, \xi)=N\left(1-\kappa \frac{\chi}{\Omega}\right) \exp \left(-2 \alpha\left(\xi+\chi \frac{\omega}{k}\right)\right) \text {. }
$$

Thus, recalling (32.8) and (32.10) one has

$$
\begin{aligned}
& \xi+\chi \frac{\omega}{k}=\frac{1}{2}\left(v_{x}^{2}+\left(v_{y}+v_{D}\right)^{2}-\left(\frac{\omega}{k}\right)^{2}\right)-g x+\frac{q}{m} \varphi_{k} \exp (i k y)\left(\frac{J_{0}^{2}}{v_{D}-\omega / k} \frac{\omega}{k}+1\right), \\
& 1-\frac{\kappa \chi}{\Omega}=1-\kappa\left(x+\frac{v_{y}}{\Omega}+\left(v_{D}-\frac{\omega}{k}\right) \frac{1}{\Omega}+\frac{q \varphi_{k} e^{i k y} J_{0}^{2}}{m \Omega\left(v_{D}-\omega / k\right)}\right) .
\end{aligned}
$$

These expressions are substituted in $f(\xi, \chi)$ and the resulting expression is expanded up to second order in $\varphi_{k}$. We can put $y=0$, for simplicity, and $J_{0}^{2}\left(k v_{\perp} / \Omega\right)=1-\tilde{b}$ where $\tilde{b}=(1 / 2)\left(k v_{\perp} / \Omega\right)^{2}$. Then one has

$$
\begin{aligned}
& f(\xi, \kappa)=N \exp \left(-\alpha\left(v_{x}^{2}+\left(v_{y}+v_{D}\right)^{2}\right)-2 \alpha g x\right)\left(1-\kappa\left(x+\frac{v_{y}}{\Omega}+\left(v_{D}-\frac{\omega}{k}\right) \frac{1}{\Omega}+\frac{q \varphi_{k}(1-\tilde{b})}{m \Omega\left(v_{D}-\omega / k\right)}\right)\right) . \\
& \cdot\left(1-\frac{2 \alpha q \varphi_{k}}{m} \frac{v_{D}-\omega \tilde{b} / k}{v_{D}-\omega / k}+2\left(\frac{\alpha q \varphi_{k}}{m}\right)^{2} \frac{v_{D}^{2}-2 v_{D} \tilde{b} \omega / k}{\left(v_{D}-\omega / k\right)^{2}}\right) .
\end{aligned}
$$

Here a term $(\omega / k)^{2}$ has been absorbed in the normalization constant $N$. We can neglect $v_{D}$ in the parenthesis $\left(v_{y}+v_{D}\right)^{2}$ because in the subsequent integration in velocity space it gives rise to the negligible term $k v_{D} / \Omega<<1$. Then the argument of the exponential reduces to $-\alpha v_{\perp}^{2}$. Terms of the form $\kappa v_{y} \tilde{b} / \Omega$ do not give a contribution to the integration in velocity space and $\left(v_{D}-\omega / k\right)(1 / \Omega)<<1, \alpha v_{D}^{2}<<1$ are neglected. We maintain only terms linear in $\tilde{b}$.

We recall that, while the function $\sigma_{m}\left(\varphi_{m}\right)$ is calculated assuming a linear approximation for the equation of the orbits in the procedure for the solution of Vlasov equation in view of $\varphi_{m} \rightarrow 0$, the quantity $\varphi_{k}$, which describes a fluctuation outside the Vlasov equilibrium, can be taken also at higher orders because it is not required to be a solution of Vlasov equation (32.11) at second order (see sections 28-30). Note also that the function $\sigma_{m}=\sigma_{m}\left(\varphi_{m}\right)$ is evaluated with $\varphi_{m} \rightarrow 0$ and therefore the normalization constant $N$ must be calculated in this limit. For a generic $k$, one has up to second order

$$
\begin{aligned}
& \int_{0}^{\infty} f(\xi, \chi) v_{\perp} d v_{\perp}=n e^{2 \alpha g x}\left(1+q \varphi_{k}\left(\frac{2 \alpha\left(\omega b-v_{D} k\right)}{v_{D} k-\omega}+\kappa \frac{1-b}{\Omega m\left(v_{D}-\omega / k\right)}\right)\right)- \\
& -\left(\frac{q \varphi_{k}}{m}\right)^{2} \frac{2 \alpha}{\left(v_{D}-\omega / k\right)^{2}}\left(\frac{2 \alpha v_{D} \omega}{k}-\frac{\kappa}{\Omega}\left(v_{D}+b\left(v_{D}+\omega / k\right)\right)\right),
\end{aligned}
$$


where $N \int_{0}^{\infty} \exp \left(-\alpha v_{\perp}^{2}\right) \nu_{\perp} d v_{\perp}=n, b=k^{2} / 2 \alpha \Omega^{2}$.

\section{3-The coefficients $k_{m}^{2}$ and $k_{m, 2}^{2}$}

We are now in position to determine the two basic coefficients $k_{m}^{2}=4 \pi d \sigma_{m} / d \varphi_{m}$ and $k_{m, 2}^{2}=4 \pi d^{2} \sigma_{m} / d \varphi_{m}^{2}$ (see eq. (28.2). The coefficient $k_{m}^{2}$ determines the marginal equilibrium through the relation $\varepsilon_{m}=1-k_{m}^{2} / k^{2}=0$ (see eq. (27.2)) and $k_{m, 2}^{2}$ determines the mean square average of the thermodynamic fluctuations according to eqs. (30.10) and (30.11).

We assume equal temperatures for ions and electrons, thus $T_{e}=T_{i}=T, \alpha_{i} m_{e}=\alpha_{e} m_{i}, v_{D e}=-g_{e} / \Omega_{e}=g_{i} / \Omega_{i}=-v_{D i}, \alpha_{e} \Omega_{e}=-\alpha_{i} \Omega_{i}, \alpha_{e} g_{e}=\alpha_{i} g$.

We assume zero Larmor radius for the electrons, $b_{e}=0$, while $b \equiv b_{i}=k^{2} / 2 \alpha_{i} \Omega_{i}^{2}=k^{2} \rho_{i}^{2} / 2$ where $\rho_{i}$ is the Larmor radius of the ions.

The charge density of the electrons $\left(b_{e}=0, q=-e\right)$ at first order is then

$\sigma_{1 e}=n e^{2 \alpha g x} e^{2} \varphi_{k}\left(\frac{2 \alpha_{e} v_{D e} k}{m_{e}\left(\omega-v_{D e} k\right)}+\frac{\kappa}{m_{e} \Omega_{e}\left(v_{D e}-\omega / k\right)}\right)$,

and that of the ions $(b \neq 0, q=e)$

$\sigma_{1 i}=n e^{2 g \alpha x} e^{2} \varphi_{k}\left(\frac{2 \alpha_{i}}{m_{i}} \frac{v_{D i} k-\omega b}{\omega-v_{D i} k}+\frac{\kappa(1-b)}{m_{i} \Omega_{i}\left(v_{D i}-\omega / k\right)}\right)$.

After some algebra one finds the following expression for the total charge density $\sigma_{1}=\sigma_{1 e}+\sigma_{1 i}$ at first order

$$
\sigma_{1}=e^{2} n \varphi_{k} e^{2 \alpha g x} \frac{2 \alpha_{e}}{m_{e}} \frac{1}{\omega^{2}-k^{2} v_{D}^{2}}\left(-b\left(\omega^{2}-\omega\left(\frac{\kappa k T C}{e B}+k v_{D e}\right)+2 \kappa g_{i}-\frac{\kappa k^{2} v_{D e}}{\alpha_{e} \Omega_{e}}\right)+2 v_{D e} k^{2}\right) \text {. }
$$

It will turn out that $v_{D}<<\omega / k$, so the terms in $v_{D}$ are negligible. In this limit the linear dielectric constant becomes (noting that $x / R_{c}<<1$ and then $e^{2 \alpha g x} \approx 1$ )

$\varepsilon_{m}\left(k, k_{m}, \omega_{m} \kappa\right)=1-\frac{k_{m}^{2}}{k^{2}} \approx \frac{8 \pi n e^{2}}{k^{2} \omega_{m}^{2}} \frac{\alpha}{m} b_{m}\left(\omega_{m}^{2}-\omega_{m} \frac{\kappa k_{m} T c}{e B}+2 \kappa g_{i}\right)=0$.

where $k_{m}^{2}=4 \pi\left(\sigma_{1} / \varphi_{k}\right)_{k=k_{m}, \omega=\omega_{m}}$ with $\sigma_{m}, \varphi_{m} \rightarrow 0$. One obtains, in the limit $k^{2} \lambda_{D}^{2}<<1$ implied by the second transition in (32.21), the dispersion relation of the flute modes with finite Larmor radius correction (see e.g. [38]).

We now consider the charge density at second order:

$$
\begin{aligned}
\sigma_{2 e} & =n e^{3} \varphi_{k}^{2} \frac{2 \alpha_{e} \kappa v_{D e}}{\Omega_{e} m_{e}^{2}\left(v_{D e}-\omega / k\right)^{2}}, \\
\sigma_{2 i} & =n e^{3} \varphi_{k}^{2}\left(\frac{2 \alpha_{i}}{m_{i}^{2}\left(v_{D e}+\omega / k\right)^{2}}\left(-b \frac{\kappa}{\Omega_{i}}\left(v_{D e}+\frac{\omega}{k}+2 \alpha_{i} v_{D e} \frac{\omega}{k}\right)+\frac{\kappa v_{D e}}{\Omega_{i}}\right)\right) .
\end{aligned}
$$

Neglecting terms $v_{D}<<\omega / k$ the coefficient $k_{m, 2}^{2}=\left(8 \pi / \varphi_{m}^{2}\right)\left(\sigma_{2 e}+\sigma_{2 i}\right)_{m}$ is written as $k_{m, 2}^{2}=-16 \pi n e^{3}\left(\frac{\alpha_{i} k_{m}^{2}}{m_{i}^{2} \omega_{m}^{2}} b_{m}\left(\frac{\kappa v_{D e}}{\Omega_{i}}+\frac{2 \alpha_{i} v_{D e} \omega_{m}}{k_{m}}+\frac{\kappa \omega_{m}}{\Omega_{i} k_{m}}\right)\right)$, 
where $k_{m}$ and $\omega_{m}$ satisfy the dispersion relation (32.21). According to this relation the instability exists in the following range of $\kappa R_{c}$

$2<R_{c} \kappa<\frac{32}{\left(\rho_{i} k_{m}\right)^{2}}$.

The lower limit arises from consistency with $\omega_{m}^{2}>>k_{m}^{2} v_{D}^{2}$. The upper limit is the bifurcation point determined by $\varepsilon_{m}=0, \partial \varepsilon_{m} / \partial \omega_{m}=0$ :

$$
R_{c, m} \kappa=\frac{32}{\left(\rho_{i} k_{m}\right)^{2}}, \quad \omega_{m}=\left(2 g_{i, m} \kappa\right)^{1 / 2}, \quad g_{i, m}=\frac{v_{t h, i}^{2}}{R_{c, m}} .
$$

The consistency with $\left(\rho_{i} k_{m}\right)^{2}<<1$ implies that $R_{c, m}$ is very large, i.e. $R_{c}$ is precisely near the boundary $R_{c, m} \rightarrow \infty$ between favorable and unfavorable curvature where $R_{c}$ changes sign trough infinity.

Taking into account the relations above one finds that the first three terms of (32.23) are negligible with respect to the last term in view of the inequality $v_{D e}<<\omega / k$. Thus only the last term survives:

$k_{m, 2}^{2}=-\frac{16 \pi n e^{3} \alpha_{i} k_{m} \kappa}{m_{i}^{2} \Omega_{i} \omega_{m}} b_{m}=-\frac{16 \pi n e^{3} k_{m} \alpha_{i}\left(\kappa R_{c, m}\right)^{1 / 2}}{(2)^{1 / 2} m_{i}^{2} \Omega_{i} v_{i, t h}} b_{m}=-\frac{64 \pi n e^{3} \alpha_{i}}{m_{i}^{2} \Omega_{i} v_{i, t h} \rho_{i}} b_{m}=-\frac{16 \pi n e^{3}}{T^{2}} b_{m}$.

Insertion into (30.10) gives the fluctuation level for the mode $k_{m}$ at the marginal point $\varepsilon_{m}=0$ :

$\frac{e^{2} \overline{\varphi^{2}}}{T^{2}}=\frac{2}{n}\left(\frac{1}{3 \pi}\right)^{1 / 2}\left(\sum_{s} \frac{n_{s}^{2}}{N_{s}}\right)^{1 / 2} \frac{1}{b_{m}}=\left(\frac{2}{3 \pi}\right)^{1 / 2} \frac{4}{N_{s}^{1 / 2} k^{2} \rho_{i}^{2}}$.

Insertion into (30.11) with $\varepsilon_{m}=-1$ gives the large amplitude of the fluctuating single $k=k_{m} /(2)^{1 / 2}$-mode corresponding to the absolute maximum of the entropy in the linearly unstable region:

$\left(\frac{e^{2} \overline{\varphi^{2}}}{T^{2}}\right)_{\max }=\frac{8}{3} k_{m}^{4} \frac{T^{2}}{\left(16 \pi e^{2} n_{0} b_{m}\right)^{2}}=\frac{2}{3} \frac{\lambda_{D e}^{4}}{\rho_{i}^{4}}=\frac{1}{6}\left(\frac{\Omega_{i}}{\omega_{i, p}}\right)^{4}$,

where $\omega_{i, p}^{2}=4 \pi n e^{2} / m_{i}$.

The example above serves as illustration of the method for calculating the amplitude of the thermodynamic fluctuations of the charge density in reactive electrostatic systems. In practice the simple situation considered above is modified by a number of important effects, in particular by the presence of magnetic shear, by effects related to the propagation parallel to the magnetic field ( $k_{\|} \neq 0$, drift modes, electromagnetic interchange modes) and by the finite toroidal geometry of practical devices (see e.g. [38]).

\section{CHAPTER 11: Connexion between the electrostatic entropy and the Lagrangian description of the particle motion}

Summary: In this chapter the relation between the electrostatic entropy and the Lagrangian description of motion of the high temperature system of Coulomb interacting particles is investigated.

The global system is considered as isolated and the motion is constrained by the macroscopic condition that the total electrostatic energy $U$ of the interacting particles belonging to different volume elements $\Delta V_{j}$ is fixed. The quantity $U$ is the mutual electrostatic energy of the system of particles 
when the charges of the particles are "smeared out" in $\Delta V_{j}$. Thus the Lagrangian contains the term $U$ multiplied by a Lagrangian multiplier $\lambda$.

Proceeding as in the magnetic case treated in chapter 7, we look first for a linear relation between the canonical average of the Lagrangian and the electrostatic entropy, as well as between their variations.

The canonical average involves the consideration of a coarse-grained system partitioned into volume elements $\Delta V_{j}$ inside which the positions $\vec{x}_{n}$ and $\vec{x}_{n^{\prime}}$ of particles $n$ and $n^{\prime}$ at a given instant of time are not distinguishable. The relation with the entropy arises from a term that expresses this fact. The Lagrange multiplier $\lambda$ turns out to be related to the degree of coarse-graining.

As a consequence of the macroscopic constraint imposed on the system by fixing $U$ and of the relation of $\lambda$ with the coarse-graining, the Lagrange equations of motion imply the equations of motion at the individual particle level and, at the same time, that the momentum of the global system is constant in time and that the system is electrically neutral globally.

Under the hypothesis that the canonical average of the first variation of the Lagrangian is equal to its time average, one finds equivalence between the Hamilton's action principle and the vanishing of the first variation of $S_{p}$ with respect to adiabatic variations.

\section{Coarse graining of the high temperature system of Coulomb interacting particles}

We consider a system of $N_{p}$ particles interacting through a Coulomb potential $W(t)=\frac{1}{2} \sum_{n \neq n^{\prime}}^{N_{p}} \frac{e_{n} e_{n^{\prime}}}{\vec{x}_{n}(t)-\vec{x}_{n^{\prime}}(t)}$.

Let us introduce a partition of the volume $V$ of the system of particles into $N$ volume elements $\Delta V_{j}$ each containing, at a given instant of time $t=t_{0}$, a large number of particles $N_{j}$. We assume that the temperature is so high that the Debye length $\lambda_{D}=\left(T / 4 \pi n e^{2}\right)^{1 / 2}$ is larger than the edge $l=\left(\Delta V_{j}\right)^{1 / 3}$, in spite of the fact that $\Delta V_{j}$ should be sufficiently large for containing many particles. Thus the particles in $\Delta V_{j}$ can be treated as effective free particles and their number, as well as the total electric charge can be considered as fluctuating at random. Applying the partition above the expression (33.1) at $t=t_{0}$ takes on the form

$W\left(t_{0}\right)=\frac{1}{2} \sum_{j=1}^{N} \sum_{n \neq n^{\prime}}^{N_{j}} \sum_{j^{\prime}=1}^{N} \sum_{n^{\prime}=1}^{N_{j^{\prime}}} \frac{e_{n} e_{n^{\prime}}}{\left|\vec{x}_{n}\left(t_{0}\right)-\vec{x}_{n^{\prime}}\left(t_{0}\right)\right|}$.

The coarse-graining consists in the approximation of considering as indistinguishable the positions $x_{n}$ and $x_{n^{\prime}}$ of particles in the same $\Delta V_{j}$. We express this by taking in the Fourier expansion of $\left|\vec{x}_{n}-\vec{x}_{n^{\prime}}\right|^{-1}$ only the wavelengths larger than $2 l$. The $k$-summation is then cut-off at $k_{M}=\pi / l$. The positions of the particles in $\Delta V_{j}$ can be identified with any chosen value $X_{j}$ in $\Delta V_{j}$, for instance with the average

$\vec{X}_{j}=\frac{1}{N_{j}} \sum_{n=1}^{N_{j}} \vec{x}_{n}\left(t_{0}\right)$

In particular one can no longer distinguish between $n=n^{\prime}$ and $n \neq n^{\prime}$ in the same $\Delta V_{j}$ because the concept of a point particle looses its meaning in the coarse-graining and one has to deal with a 
"smeared out" charge rather than with point charges. In this point of view $W\left(t_{0}\right)$ can depend only on the $\vec{X}_{j}$ and on the total charge $\sum_{n=1}^{N_{j}} e_{n}$ in each $\Delta V_{j}$. The Fourier representation of $W\left(t_{0}\right)$ is then the following:

$W\left(t_{0}\right)=\frac{1}{2} \sum_{j=1}^{N} \sum_{n=1}^{N_{j}} e_{n} \sum_{j^{\prime}=1}^{N} \sum_{n^{\prime}=1}^{N_{j^{\prime}}} e_{n^{\prime}} \frac{4 \pi}{V} \sum_{-k_{M}}^{k_{M}} \frac{1}{k^{2}} \exp \left(-i \vec{k} \cdot\left(\vec{X}_{j}-\vec{X}_{j^{\prime}}\right)\right)$

It is convenient to separate the terms with $j=j^{\prime}$ from the terms $j \neq j^{\prime}$,

$W\left(t_{0}\right)=\frac{1}{2} \sum_{j=1}^{N} \sum_{n=1}^{N_{j}} e_{n} \sum_{j=1}^{N} \sum_{n^{\prime}=1}^{N_{j}} e_{n^{\prime}} \frac{4 \pi}{V} \sum_{-k_{M}}^{k_{M}} \frac{1}{k^{2}}+U\left(t_{0}\right)$,

where

$U\left(t_{0}\right)=\frac{1}{2} \sum_{j \neq j^{\prime}}^{N} \sum_{n}^{N_{j}} e_{n} \sum_{j^{\prime}=1}^{N} \sum_{n^{\prime}=1}^{N_{j^{\prime}}} e_{n^{\prime}} \frac{4 \pi}{V} \sum_{-k_{M}}^{k_{M}} \frac{1}{k^{2}} \exp \left(-i \vec{k} \cdot\left(\vec{X}_{j}-\vec{X}_{j^{\prime}}\right)\right)$

In this expression one has $\left|\vec{X}_{j}-\vec{X}_{j^{\prime}}\right|>l$ and even $\left|\vec{X}_{j}-\vec{X}_{j^{\prime}}\right|>>l$ for the majority of the terms of the $j \neq j^{\prime}$ summation. It follows that the cut-off at $k_{M}$ is ineffective in (33.6) because the contribution of terms with $k>k_{M}$ is sensible only for $\left|\vec{X}_{j}-\vec{X}_{j^{\prime}}\right|<l$, which is not the case of (33.6). So $k_{M}$ can be replaced by $\infty$ and one obtains

$U\left(t_{0}\right)=\frac{1}{2} \sum_{j \neq j^{\prime}}^{N} \sum_{n}^{N_{j}} e_{n} \sum_{j^{\prime}=1}^{N} \sum_{n^{\prime}=1}^{N_{j^{\prime}}} e_{n^{\prime}} \frac{4 \pi}{V} \sum_{-\infty}^{\infty} \frac{1}{k^{2}} \exp \left(-i \vec{k} \bullet\left(\vec{X}_{j}-\vec{X}_{j^{\prime}}\right)\right)=\frac{1}{2} \sum_{j \neq j^{\prime}}^{N} \sum_{n=1}^{N_{j}} e_{n} \sum_{j^{\prime}=1}^{N} \sum_{n^{\prime}=1}^{N_{j^{\prime}}} e_{n^{\prime}} \frac{1}{\left|\vec{X}_{j}-\vec{X}_{j^{\prime}}\right|}$.

Let us divide $N_{j}$ in an average part and in a fluctuating part $N_{j}=\overline{N_{j}}+\tilde{N}_{j}$. Correspondingly the charge density can be divided in a collective part and in a fluctuating part

$\Delta V_{j} \sigma\left(\vec{X}_{j}\right)=\sum_{n=1}^{\overline{N_{j}}} e_{n}, \Delta V_{j} \tilde{\sigma}_{j}=\sum_{n=\bar{N}_{j}}^{\tilde{N}_{j}} e_{n}$.

Here $\overline{N_{j}}(1 \leq j \leq N)$ is a set of numbers whose specification determines $\sigma\left(\vec{X}_{j}\right)$ and the collective electrostatic charge density. By substituting (33.8) in (33.5) one obtains

$W\left(t_{0}\right)=\frac{1}{2} \sum_{j=1}^{N}\left(\Delta V_{j}\left(\sigma\left(\vec{X}_{j}\right)+\tilde{\sigma}_{j}\right)\right)^{2} \frac{4 \pi}{V} \sum_{-k_{M}}^{k_{M}} \frac{1}{k^{2}}+U\left(t_{0}\right)$,

where

$U\left(t_{0}\right)=\frac{1}{2} \sum_{j \neq j^{\prime}}^{N} \Delta V_{j}\left(\sigma\left(\vec{X}_{j}\right)+\tilde{\sigma}_{j}\right) \sum_{j^{\prime}=1}^{N}\left(\Delta V_{j^{\prime}}\left(\sigma\left(\vec{X}_{j^{\prime}}\right)+\tilde{\sigma}_{j^{\prime}}\right)\right) \frac{1}{\left|\vec{X}_{j}-\vec{X}_{j^{\prime}}\right|}$.

The quantity $U$ can be expressed in terms of the collective electrostatic energy $\Phi$ and of the interaction energy $\Phi_{\text {int }}$. Indeed, putting

$\varphi\left(\vec{X}_{j}\right)=\sum_{j^{\prime} \neq j}^{N} \Delta V_{j^{\prime}} \frac{\sigma\left(\vec{X}_{j^{\prime}}\right)}{\left|\vec{X}_{j}-\vec{X}_{j^{\prime}}\right|}$

one has, neglecting terms quadratic in $\tilde{\sigma}_{j}$

$U\left(t_{0}\right)=\Phi+2 \tilde{\Phi}_{\mathrm{int}}$,

where 
$\Phi=\frac{1}{2} \sum_{j=1}^{N} \varphi\left(\vec{X}_{j}\right) \partial\left(\vec{X}_{j}\right) \Delta V_{j}, \widetilde{\Phi}_{\mathrm{int}}=\frac{1}{2} \sum_{j=1}^{N} \varphi\left(\vec{X}_{j}\right) \tilde{\sigma}\left(\vec{X}_{j}\right) \Delta V_{j}$

The quantity $U$ is interpreted as the mutual electrostatic energy of the system of particles when the charges of the particles are "smeared out" in $\Delta V_{j}$.

\section{Equivalence of the first variations of the electrostatic entropy and of the canonically averaged Lagrangian with respect to adiabatic variations}

We shall study the motion of the particles under the constraint that the electrostatic energy $U$ is fixed. In particular the value of $U$ is fixed to the average $U=\Phi+\overline{2 \widetilde{\Phi}_{\text {int }}}$ calculated with the canonical distribution (25.5). The Lagrangian appropriate for the description of the motion is the following $L(t)=\frac{1}{2} \sum_{n=1}^{N_{p}} m_{n} v_{n}^{2}-W(t)-\lambda U(t)$

where $W(t)$ is given by (33.1), $U(t)$ by (33.7) (with $t_{0}$ replaced by $t$ ), the $\vec{x}_{n}(t)$ and the $\vec{X}_{j}(t)$ are time dependent and $\lambda$ is a Lagrange multiplier related to the constraint that $U$ is fixed.

The parameter $\lambda$ should be determined from the constraint $U=\bar{U}$ after solution of the equations of the constrained motion, but one can do the reverse and determine $\bar{U}$ after a proper choice of $\lambda$. We show presently that, with a proper choice of $\lambda$, one has a linear simple relation between the canonical average of the Lagrangian at a fixed time $t=t_{0}$ and the electrostatic entropy, a fact that will be relevant for connecting the variation of the Lagrangian with that of the entropy.

The sum of the single particle kinetic energy $K=(1 / 2) \sum_{n} m_{n} v_{n}^{2}$ is considered as fixed by taking, for instance, the average with a Maxwellian distribution with fixed temperature. The collective quantities are averaged with the canonical distribution (25.5):

$\overline{L\left(t_{0}\right)}=K-\overline{W\left(t_{0}\right)}-\lambda \overline{U\left(t_{0}\right)}$,

where, recalling (33.8) and (33.11),

$\overline{W\left(t_{0}\right)}=\sum_{j=1}^{N}\left(\Delta V_{j}\left(\frac{1}{2} \sigma^{2}\left(\vec{X}_{j}\right)+\sigma\left(\vec{X}_{j}\right) \overline{\widetilde{\sigma}}_{j}+\frac{1}{2} \overline{\tilde{\sigma}_{j}^{2}}\right)\right) \frac{4 \pi}{N} \sum_{-k_{M}}^{k_{M}} \frac{1}{k^{2}}+\overline{U\left(t_{0}\right)}$,

$\overline{U\left(t_{0}\right)}=\Phi+2 \overline{\widetilde{\Phi}_{\mathrm{int}}}=\Phi+2 \Phi_{\mathrm{int}}$.

Recalling (25.10) and (25.12) one has

$$
\begin{aligned}
& \int_{\Omega} \sigma^{2}(\vec{X}) d \Omega=-2 \Delta V \overline{\tilde{\sigma}^{2}} S_{p}+\frac{k_{m}^{2}}{4 \pi} \int_{\Omega} \sigma(\vec{X}) \varphi(\vec{X}) d \Omega, \\
& \int_{\Omega} \sigma(\vec{X}) \bar{\sigma}(\vec{X}) d \Omega=2 \Delta V \overline{\tilde{\sigma}^{2}} S_{p} .
\end{aligned}
$$

Insertion of (34.4) into (34.3) (replacing the $j$-summation with the integral) gives

$\overline{W\left(t_{0}\right)}+\lambda \overline{U\left(t_{0}\right)}=\left(\Delta V \overline{\tilde{\sigma}^{2}} S_{p}+\frac{k_{m}^{2}}{8 \pi} \int \sigma \varphi d \Omega+\frac{1}{2} \sum_{j=1}^{N} \Delta V_{j} \overline{\tilde{\sigma}_{j}^{2}}\right) \frac{4 \pi}{N} \sum_{-k_{M}}^{k_{M}} \frac{1}{k^{2}}+\bar{U}(1+\lambda)$.

We note that the term involving the entropy arises from the term in (34.3) that depends on $k_{M}$ and expresses that the positions of the particles in the same $\Delta V_{j}$ are undistinguishable.

Let us put 
$\lambda_{1}=\frac{k_{m}^{2}}{N} \sum_{-k_{M}}^{k_{M}} \frac{1}{k^{2}}, \lambda=-1-\lambda_{1}$.

Then

$\overline{L\left(t_{0}\right)}=K-\lambda_{1} \frac{2 \pi V \overline{\tilde{\sigma}^{2}}}{k_{m}^{2}}-\lambda_{1} \frac{4 \pi \Delta V \overline{\tilde{\sigma}^{2}}}{k_{m}^{2}} S_{p}+2 \lambda_{1} \Phi_{\text {int }}$.

Finally, recalling (25.11) and (26.1), one obtains

$\overline{L\left(t_{0}\right)}=K+\lambda_{1} \frac{N \tau}{2}+\lambda_{1}\left(\tau S+\Phi_{\text {int }}\right)$,

where $S=S_{p}+S_{b}$ and $S_{b}=\Phi_{\text {int }} / \tau$.

The variation of the collective quantities with $\tau$ fixed (isothermal variations, see section 26 ) gives $\overline{\delta L\left(t_{0}\right)}=\lambda_{1}\left(\tau \delta S+\delta \Phi_{\text {int }}\right)$.

The condition $\overline{\delta L}=0$ implies $\tau \delta S+\delta \Phi_{\text {int }}=0$. In the case of adiabatic variations (in the sense of section 9) one has $\delta \varphi=\left(4 \pi / k_{m}^{2}\right) \delta \sigma$ around the Vlasov equilibrium $\varphi_{m}=\left(4 \pi / k_{m}^{2}\right) \sigma_{m}$. Then one has separately $\delta S=0, \delta \Phi_{\text {int }}=0$ (section 26). It follows that $\overline{\delta L}=0$ and $\delta S_{p}=\tau \delta Q=0$ are equivalent with respect to reversible adiabatic variations. The significance of this relation will be clarified in the next section.

To conclude this section let us evaluate the Lagrange multiplier $\lambda$ given by (34.6). In the limit $V \rightarrow \infty$ the expression (34.6) for $\lambda_{1}$ becomes

$\lambda_{1}=\frac{k_{m}^{2}}{N} \sum_{-k_{M}}^{k_{M}} \frac{1}{k^{2}} \rightarrow \frac{k_{m}^{2} V}{(2 \pi)^{2} N} \int_{0}^{k_{M}} d k \int_{0}^{\pi} \sin \theta d \theta=\frac{k_{m}^{2} k_{M} \Delta V}{2 \pi^{2}}$

where $k_{M}=\pi / l$. Introducing the characteristic length of the Vlasov equilibrium $\ell=\left|\pi / k_{m}\right|$ one has the inequality

$\left|\lambda_{1}\right|=\frac{1}{2}\left(\frac{l}{\ell}\right)^{2}<<1$

We see that the parameter $\lambda_{1}$, although finite, is very small. It follows that $\lambda$ is very close to -1 but not identical to it.

\section{Particle motion in the electrostatic system with the collective constraint and the relation of the first variation of the entropy to the action principle}

We now proceed to the derivation of the equations of the constrained motion according to the Lagrangian (34.1). The application of the Hamilton’s action principle gives

$\int_{t_{1}}^{t_{2}} \delta L d t=\int_{t_{1}}^{t_{2}} \sum_{n=1}^{N_{p}}\left(\frac{\partial}{\partial \vec{x}_{n}}-\frac{d}{d t} \frac{\partial L}{\partial \vec{\gamma}_{n}}\right) \cdot \delta \vec{x}_{n}(t) d t=0$,

and in view of the arbitrariness of the $\delta \vec{x}_{n}(t)$ (vanishing at $t_{1}$ and $t_{2}$ ) one obtains the equation of motion for the generic $s$-particle:

$\frac{\partial}{\partial \vec{x}_{s}}-\frac{d}{d t} \frac{\partial L}{\partial \vec{v}_{s}}=0$

The following equalities will be applied: 


$$
\begin{aligned}
& e_{s} \vec{\varepsilon}\left(\vec{x}_{s}\right) \equiv-\nabla_{x_{s}} W=-\frac{1}{2} \nabla_{x_{s}} \sum_{n \neq n^{\prime}}^{N_{p}} \frac{e_{n} e_{n^{\prime}}}{\left|\vec{X}_{n}-\vec{x}_{n^{\prime}}\right|}=-\nabla_{x_{s}} \sum_{n \neq s}^{N_{p}} \frac{e_{s} e_{n}}{\left|\vec{x}_{n}-\vec{x}_{s}\right|}=\sum_{n \neq s}^{N_{p}} \frac{e_{s} e_{n}\left(\vec{x}_{s}-\vec{x}_{n}\right)}{\left|\vec{X}_{s}-\vec{x}_{n}\right|^{3}}, \\
& E\left(\vec{X}_{j_{s}}\right) \equiv-\sum_{j \neq j_{s}}^{N} \sum_{n=1}^{N} e_{n} \nabla_{x_{s}} \frac{1}{\left|\vec{X}_{j_{s}}-\vec{X}_{j}\right|} .
\end{aligned}
$$

Indeed, we start by showing the relation

$$
\nabla_{x_{s}} U=\frac{1}{N_{j_{s}}} \nabla_{X_{s}} U=-\frac{1}{N_{j_{s}}}\left(\sum_{n=1}^{N_{j_{s}}} e_{n}\right) \vec{E}\left(\vec{X}_{j}\right) \text {, }
$$

where $N_{j_{s}}$ is the group of particles that contains the $s$-particle defined by the position $\vec{X}_{s}\left(t_{0}\right)$ in $\Delta V_{j_{s}}$. For simplicity of notation we put $j_{s}=S, \vec{X}_{j_{s}}=\vec{X}_{S}, N_{j_{s}}=N_{s}, \Delta V_{j_{s}}=\Delta V_{S}$. We also put $Q_{j}=\sum_{1}^{N_{j}} e_{n}$, so that the expression (33.7) for $U$ (where $t_{0}$ is replaced by $t$ ) becomes

$U(t)=\frac{1}{2} \sum_{j \neq j^{\prime}}^{N} Q_{j} \sum_{j^{\prime}=1}^{N} Q_{j^{\prime}} \frac{1}{\left|\vec{X}_{j}(t)-\vec{X}_{j^{\prime}}(t)\right|}$.

One has

$$
\begin{aligned}
& \nabla_{x_{S}} U=\frac{1}{2 N_{S}} \nabla_{X_{S}} U=\frac{1}{2 N_{S}} \nabla_{X_{S}} \sum_{j \neq j^{\prime}}^{N_{j}} Q_{j} \sum_{j^{\prime}=1}^{N_{j^{\prime}}} Q_{j^{\prime}} \frac{1}{\left|\vec{X}_{j}-\vec{X}_{j^{\prime}}\right|}=\frac{Q_{S}}{N_{S}} \sum_{j \neq S}^{N_{j}} Q_{j} \nabla_{X_{S}} \frac{1}{\left|\vec{X}_{j}-\vec{X}_{S}\right|}= \\
& \frac{1}{N_{S}} \sum_{n=1}^{N_{S}} e_{n} \sum_{j \neq S}^{N} \sum_{n^{\prime}=1}^{N_{j}} e_{n^{\prime}} \nabla_{X_{S}} \frac{1}{\left|\vec{X}_{j}-\vec{X}_{S}\right|}=-\frac{1}{N_{S}} \sum_{n=1}^{N_{S}} e_{n} \vec{E}\left(\vec{X}_{j}\right) .
\end{aligned}
$$

Applying these relations, the equation of motion (35.2) for the $s$-particle takes the form $m_{s} \frac{d}{d t} \vec{v}_{s}=e_{s} \vec{\varepsilon}\left(\vec{X}_{s}\right)+\lambda \frac{Q_{S}}{N_{s}} \vec{E}\left(\vec{X}_{s}\right)$

For seeing the meaning of this equation let us sum up with respect to all the $N_{S}$ particles in $\Delta V_{S}$ at the initial time $t_{0}$

$\sum_{s=1}^{N_{S}} m_{s} \frac{d}{d t} \vec{v}_{s}=\sum_{1}^{N_{S}} e_{s} \vec{\varepsilon}\left(\vec{x}_{s}\right)+\lambda Q_{S} \vec{E}\left(\vec{X}_{S}\right)$

The following equality holds:

$\sum_{1}^{N_{S}} e_{s} \vec{\varepsilon}\left(\vec{x}_{s}\right)=Q_{S} \vec{E}\left(\vec{X}_{S}\right)$

Indeed, one can write

$\sum_{s=1}^{N_{S}} e_{s} \vec{\varepsilon}\left(\vec{x}_{s}\right)=\sum_{n \neq s}^{N_{p}} e_{n} \sum_{s=1}^{N_{s}} e_{s} \frac{\vec{x}_{s}-\vec{x}_{n}}{\left|\vec{x}_{s}-\vec{x}_{n}\right|^{3}}=\sum_{n \neq s}^{N_{S}} e_{n} \sum_{s=1}^{N_{S}} e_{s} \frac{\vec{x}_{s}-\vec{x}_{n}}{\left|\vec{x}_{s}-\vec{x}_{n}\right|^{3}}+\sum_{j \neq s}^{N} \sum_{n=1}^{N_{j}} e_{n} \sum_{s=1}^{N_{S}} e_{s} \frac{\vec{x}_{s}-\vec{x}_{n}}{\left|\vec{x}_{s}-\vec{x}_{n}\right|^{3}}$.

The first term in the right hand side vanishes in view of the antisymmetry of the term under the sum. The last term is formed by summations over groups of particles that are contained at the instant $t_{0}$ inside volume elements with $j \neq j_{S}$. In the coarse-grained approximation one can replace $\vec{x}_{s}$ and $\vec{x}_{n}$ with the averages $\vec{X}_{S}$ and $\vec{X}_{j}$ in $\Delta V_{S}$ and $\Delta V_{j}$ obtaining

$\sum_{s=1}^{N_{S}} e_{s} \vec{\varepsilon}\left(\vec{X}_{s}\right)=\sum_{s=1}^{N_{S}} e_{s} \sum_{j \neq S}^{N} \sum_{n=1}^{N_{j}} e_{n} \frac{\vec{X}_{s}-\vec{X}_{n}}{\left|\vec{X}_{s}-\vec{X}_{n}\right|^{3}}=-Q_{S} \sum_{j \neq S}^{N} \sum_{n=1}^{N_{j}} e_{n} \nabla_{X_{s}} \frac{1}{\left|\vec{X}_{s}-\vec{X}_{n}\right|}=Q_{S} \vec{E}\left(\vec{X}_{S}\right)$.

Recalling from section 33 that $\lambda=-\lambda_{1}-1$ one arrives at the relation 
$\sum_{s=1}^{N_{p}} m_{s} \frac{d}{d t} \vec{v}_{s}=-\lambda_{1} Q_{S} \vec{E}\left(\vec{X}_{S}\right)$

We know from section 34 that $\lambda_{1}$ is close to 0 but not identical to it and then (35.12) would imply that the time derivative of the total momentum of the system is close but not identical to 0 . If we want to eliminate the unphysical dependence on the coarse-graining described by $\lambda_{1}$ while remaining consistent with the result above, we are forced to admit that the two members of (35.12) must vanish separately

$\sum_{s=1}^{N_{p}} m_{s} \frac{d}{d t} \vec{v}_{s}=0, \quad Q_{S} \vec{E}\left(\vec{X}_{S}\right)=0$

It follows that the total momentum is constant in time and that the collective system is globally electrically neutral.

The reasoning above leading to (35.13), can be repeated for any group $N_{S}$ of particles in any $\Delta V_{S}$ at any time $t=t_{0}$, so we conclude that the collective system is always described by eqs.(35.13).

Using (35.13) in (35.7) one obtains the equation

$m_{s} \frac{d}{d t} \vec{v}_{s}=e_{s} \vec{\varepsilon}\left(\vec{x}_{s}\right)$,

which describes the motion of the s-particle in the field of all other particles. The solution of this equation has been treated by Pines and Bohm [2] in the case of an electron gas, assuming that phase factors, which depend on the position of the particles, average out to zero, due to the randomness of the positions (the so-called random phase approximation). It has been shown by Pines and Bohm, that this approximation holds for thermal plasmas. In this way one can derive from (35.14) the plasma oscillations at the frequency $\omega_{p}=\left(4 \pi n e^{2} / m\right)^{1 / 2}$.

Deviations from electric neutrality arise in the presence of fluctuations of the electric field around the global neutral equilibrium. We shall see that the electrostatic force associated with the fluctuation of the electric field can be compensated by the fluctuation of the pressure.

The pressure gradient is introduced through an inhomogeneous term in the action principle, as in section 22:

$\int_{t_{1}}^{t_{2}} \delta L d t=\int_{t_{1}}^{t_{2}} \sum_{n=1}^{N_{p}}\left(\frac{\partial L}{\partial \vec{x}_{n}}-\frac{d}{d t} \frac{\partial L}{\partial \vec{x}_{n}}\right) \cdot \delta \vec{x}_{n}(t) d t=-\lambda_{1} \int_{t_{1}}^{t_{2}} \sum_{j=1}^{N} \sum_{n=1}^{N_{j}} \frac{\nabla P\left(\vec{X}_{j}\right)}{n\left(\vec{X}_{j}\right)} \cdot \delta \vec{x}_{n}(t) d t$,

where $n\left(\vec{X}_{j}\right)=N_{j} / \Delta V_{j}$ is uniform in $\Delta V_{j}$. The coefficient $\lambda_{1}$ in the right hand side has been chosen in order that the pressure force be of the same order of the electrostatic force, consistently with (35.12).

Following the same procedure as above one arrives at the equality $\sigma\left(\vec{X}_{S}\right) \vec{E}\left(\vec{X}_{S}\right)-\nabla_{X_{S}} P\left(\vec{X}_{S}\right)=0$,

where $\sigma\left(\vec{X}_{s}\right)=\left(1 / \Delta V_{s}\right) \sum_{s=1}^{N_{s}} e_{s}$. Taking $P=P_{0}+\delta P$, where $P_{0}$ corresponds to the uniform neutral equilibrium and $\delta P$ is the pressure fluctuation that balances the electrostatic fluctuating force, one has $-\sigma\left(\vec{X}_{S}\right) \nabla_{X_{s}} \varphi=\nabla_{X_{S}} \delta P$.

In the case of fixed temperature considered above one can write $\delta P=T \delta n$ for the Maxwellian plasma and from (35.16) one gets (noting that $\sigma\left(\vec{X}_{S}\right)$ is uniform in $\left.\Delta V_{S}\right)$ 
$\sum_{s=1}^{N_{S}} e_{s} \varphi \approx \Delta V_{S} \delta P=\Delta V_{S} T \delta n=T \delta N_{S}$

Introducing the average charge in $\Delta V_{S}, \bar{e}=\left(1 / N_{S}\right) \sum_{s=1}^{N_{S}} e_{s}$ one arrives at the relation $\frac{\bar{e} \varphi}{T}=\frac{\delta N_{S}}{N_{S}}$,

which shows that the fluctuation $\varphi$ is related to the fluctuation of the number of particles in $\Delta V_{s}$.

We finally return to the basic relation (34.9),

$\overline{\delta L\left(t_{0}\right)}=\lambda_{1}\left(\tau \delta S+\delta \Phi_{\text {int }}\right)=0$,

and note that, under the hypothesis that the average $\overline{\delta L\left(t_{0}\right)}$ is equal to the time average $\left(1 /\left(t_{2}-t_{1}\right)\right) \int^{t_{2}} \delta L d t,(35.20)$ asserts the equivalence between the Hamilton's action principle and the vanishing of the first variation of $S_{p}$ with respect to adiabatic variations (which entail also the vanishing of $\delta \Phi_{\text {int }}$, see section 26). Indeed the variation of the action $\int_{t_{1}}^{t_{2}} \delta L d t=0$, as well as the variations $\delta S_{p}$ and $\delta \Phi_{\text {int }}$ can be considered as produced by the same primary variations $\delta \vec{x}_{n}(t)$ (vanishing at $t_{1}$ and $t_{2}$ ) when $\delta S_{p}$ and $\delta \Phi_{\text {int }}$ are generated by variations $\delta \sigma$ and $\delta \varphi$ defined by the equalities

$\delta \sigma=\vec{\xi} \cdot \nabla \sigma, \quad \delta \varphi=\vec{\xi} \cdot \nabla \varphi$, with $\sigma=\left(k_{m}^{2} / 4 \pi\right) \varphi$,

where in turn $\vec{\xi}$ is produced by all the $\delta \vec{x}_{n}(t)$ averaged in time

$\vec{\xi}\left(\vec{X}_{j}\right)=\frac{1}{t_{2}-t_{1}} \int_{t_{1}}^{t_{2}} \frac{1}{N_{j}} \sum_{j=1}^{N_{j}} \delta \vec{x}_{n}(t) d t$

As in section 24, the $\delta \vec{x}_{n}(t)$ are interpreted as variations of the positions of the particles in $\Delta V_{j}$ with respect to their positions $\vec{x}_{n}\left(t_{0}\right)$.

It is worthwhile to note that the condition (35.20) holds also in the case of $\overline{\delta L\left(t_{0}\right)}$ equal to the variation (35.15) of the action provided that the pressure is vanishing at infinity and the displacements $\vec{\xi}\left(\vec{X}_{j}\right)$ are incompressible. Indeed one has

$\frac{1}{t_{2}-t_{1}} \sum_{j=1}^{N} \sum_{n=1}^{N_{j}} \int_{t_{1}}^{t_{2}} \frac{\nabla P\left(\vec{X}_{j}\right)}{n\left(\vec{X}_{j}\right)} \cdot \delta \vec{x}_{n}(t) d t=\sum_{j=1}^{N} \nabla P\left(\vec{X}_{j}\right) \Delta V_{j} \cdot \frac{1}{t_{2}-t_{1}} \int_{t_{1}}^{t_{2}} \sum_{n=1}^{N} \frac{1}{N_{j}} \delta \vec{x}_{n}(t) d t=\int_{V} \vec{\xi} \cdot \nabla P d V=$
$=\int_{V} \nabla \cdot P \vec{\xi} d V-\int_{V} P \nabla \cdot \vec{\xi} d V=\int_{V} P \vec{\xi} \cdot d \vec{S}-\int_{V} P \nabla \cdot \vec{\xi} d V=0$.

(The fact that the right hand side of (35.15) is vanishing does not interfere with the variation procedure which requires the vanishing of the action with respect to each single $\delta \vec{x}_{n}(t)$; also, in spite of the condition $\nabla \cdot \vec{\xi}=0$, the $\delta \vec{x}_{n}$ remain essentially arbitrary in view of the very large value of $N_{p}$ ).

We have seen, among other things, that the descriptions at the particle level, at the collective level and at the coarse-grained level are reduced to the variation properties with respect to the variations $\delta \vec{x}_{n}$ of one single mathematical entity, the Lagrangian, which adapts flexibly to the different levels of description. 


\section{References}

1. Bohm, D.; Pines,D. A Collective Description of Electron Interactions: I. Magnetic Interactions. Phys. Rev. 1951, 82, 625-634.

2. Pines, D.; Bohm, D. A Collective Description of Electron Interactions: II. Collective vs Individual Particle Aspects of the Interactions. Phys. Rev. 1952, 85, 338-363.

3. Minardi, E. The Magnetic Entropy Concept. J. Plasma Phys. 2005, 71, 53-80.

4. Jaynes, E. T. Papers on Probability, Statistics and Statistical Physics; Rosenkrantz: DordrechtReidel, 1983.

5. Reiman, A. Taylor Relaxation in a Torus of Arbitrary Aspect Ratio and Cross Section. Phys. Fluids 1981, 24, 956-963.

6. Jensen, T.; Chu, M. S. Current Drive and Helicity Injection.Phys.Fluids 1984, 27, 2881-2885.

7. Taylor, J. B. Relaxation of Toroidal Plasmas and Generation of Reverse Magnetic Fields. Phys. Rev. Lett. 1974. 3, 1139-1141.

8. Taylor, J. B. Relaxation and Magnetic Reconnection in Plasmas. Rev. Mod. Phys. 1986, 58, 741-763.

9. Chandrasekhar, S.; Woltjer, L. On Force Free Magnetic Fields. Proc. Nat. Acad. Sci. 1958, 44, 285289.

10.Woltjer, L. Hydromagnetic Equilibrium II. Stability in the Variational Formulation. Proc. Nat. Acad. Sci. 1959, 45, 769-771.

11.Chew, G. L.; Goldberger; M. L.; Low, F.E. The Boltzmann Equation and the One-Fluid Hydromagnetic Equations in the Absence of Particle Collisions. Proc. Roy. Soc. London 1956, A236, 112-118.

12.Hazeltine, R. D.; Meiss, J. D. Plasma Confinement; Addison-Wesley Publ. Company: Redwood City (Cal.), 1991; p.79.

13.Hastie, R. J.; Taylor. J. B.; Haas, F. A. Adiabatic Invariants and the Equilibrium of Magnetically

Trapped Particles. Ann. Phys. 1967, 41, 302-338,

14.Grad, H. Velocity Gradient Instability. Phys. Fluids 1966, 9, 498-513.

15.Santini, F. Collisionless Entropy and Interchange Stability of a Representative Adiabatic

Magnetoplasma. Physica 1967, 36, 538-546.

16.Minardi, E.; Santini, F. The Thermodynamics of a Collisionless Plasma at Equilibrium in a

Magnetic Field. Physica 1967, 35, 19-28.

17.Bateman. G. MHD Instabilies; The MIT Press : Cambridge (Mass.) 1980; pp95-97.

18.Coppi, B. Non-classical Transport and the "Principle of Profile Consistency". Comments Plasma Phys. Contr. Fusion 1980, 5, 261-269.

19.Minardi, E.;Weisen, H. Stationary Magnetic Entropy in Ohmic Tokamak Plasmas: Experimental

Evidence from the TCV Device. Nucl. Fusion 2001, 41,113-130.

20.Minardi, E.; Lazzaro, E.; Sozzi, C.; Cirant, S. Profile Consistency Based on the Magnetic Entropy

Concept: Theory and Observation. Nucl. Fusion 2003, 5, 369-384.

21.Minardi, E.;Sozzi, C.; Mantica, P. Stationary Magnetic Entropy Tokamak States and Experimental Observations. Nucl. Fusion 2008, 48, 045001 (13pp).

22.Weisen, H.; Behn, R.; Furno, I.; Moret, J.-M.; Sauter, O. (TCV Team). "Profile Consistency”

Features in Shaped Sawtoothing Ohmic TCV Plasma Phys. Control. Fusion 1998, 40, 1803-1818. 
23.Weisen, H.; Behn, R.; Furno, I.; Moret, J.-M.; Sauter, O. (TCV Team) "Profile Consistency"

Features in Strongly Shaped Ohmic Tokamak Plasmas. Phys. Plasmas 1999, 6, 1-4.

24.Baker, D. R.; Rosenbluth, M. N. Density Profile Consistency and its Relation to the Transport of Trapped versus Passing Electrons in Tokamaks. Phys. Plasmas 1998, 5, 2936-2931.

25.Weisen, H.; Minardi, E. Evidence for the Role of Magnetic Entropy and Turbulent Equipartition in Stationary Ohmic Tokamak Discharges. Europhys. Lett. 2001, 56, 542-548.

26.Razumova, K.A.; Andreev, V.F.; Donné, A.J.H.; Hogeweij, G.M.D.; Lysenko, S.E.; Shelukhin, D.A.; Spakman, G.W.; Vershkov, V.A.; Zhuravlev, V.A. Link between Self-Consistent Pressure Profiles and Electron Internal Transport Barriers in Tokamaks. Plasma Phys. Control. Fusion 2006, 48,1373-1388.

27.Razumova, K.A.; Andreev, V.F.; Dnestrovskij, A.Y.; Kislov, A.Y.; Kirneval, N.A.; Lysenko, S.E.; Pavlov, D.; Poznyak, V.I.; Shafranov, T.V.; Trukhina, E.V.; Zhuravlev, V.A.; Donné, J.H.; Hogeweij, G.M.D. The Main Features of Self-Consistent Pressure Profile Formation. Plasma Phys. Control. Fusion 2008, 50, 105004 (13pp).

28.Goldstein, H. Classical Mechanics; Addison-Wesley Publ. Company; Redwood City (Cal.), 1980; p24.

29.Essén, H.; Nordmark, A. Hamiltonian of a Homogeneous two-Component Plasma. Phys. Rev. 2004, 69, 036404 (9pp).

30.Bernstein, I. B.;Trehan, S. K.; Weenink, M. P. H. Plasma Oscillations: II. Kinetic Theory of Waves in Plasmas. Nucl. Fusion 1964, 4, 61-104.

31.Bernstein, I. B.; Greene, J. M.; Kruskal. M. D. Exact Nonlinear Plasma Oscillations. Phys. Rev. 1957, 108, 546-550.

32.Cupperman, S.; Tzur, I. The Verification of Minardi’s Instability Criterion for Non-homogeneous Selfgravitating Equilibria. Astrophys. J. 1973, 180, 181-187.

33.Finzi, U.; Doremus, J. P.; Holec, J.; Feix, M. Classification and Stability of Double Water-Bag Plasma Equilibria Plasma Phys. Contrl. Fusion 1974, 16, 189-200.

34.Schwarzmeier, J. L.; Lewis, H. R.; Abraham-Schrauner, B.; Symon, K. R. Stability of BernsteinGreene-Kruskal Equilibria Phys. Fluids 1979, 22, 1747-1760.

35.Minardi, E. Fluctuation Levels Connected with Reactive Marginal Instabilities. J. Plasma Phys. 1974, 11, 471-483.

36.Abramowitz, M.; Stegun, I. A. Handbook of Mathematical Functions; Dover Publications INC. New York 1965; p298.

37.Taylor, J. B.; McNamara, B. Plasma Diffusion in Two Dimensions. Phys. Fluids 1971, 14, 14921499.

38.Weiland, J. Collective Modes in Inhomogeneous Plasma; Institute of Physics Publishing: Bristol, 2000; p42.

39.Whittaker, E. T.; Watson, G. N. Modern Analysis; Cambridge University Press: Cambridge (U.K.), 1959; p452.

(C) 2009 by the authors; licensee Molecular Diversity Preservation International, Basel, Switzerland. This article is an open-access article distributed under the terms and conditions of the Creative Commons Attribution license (http://creativecommons.org/licenses/by/3.0/). 